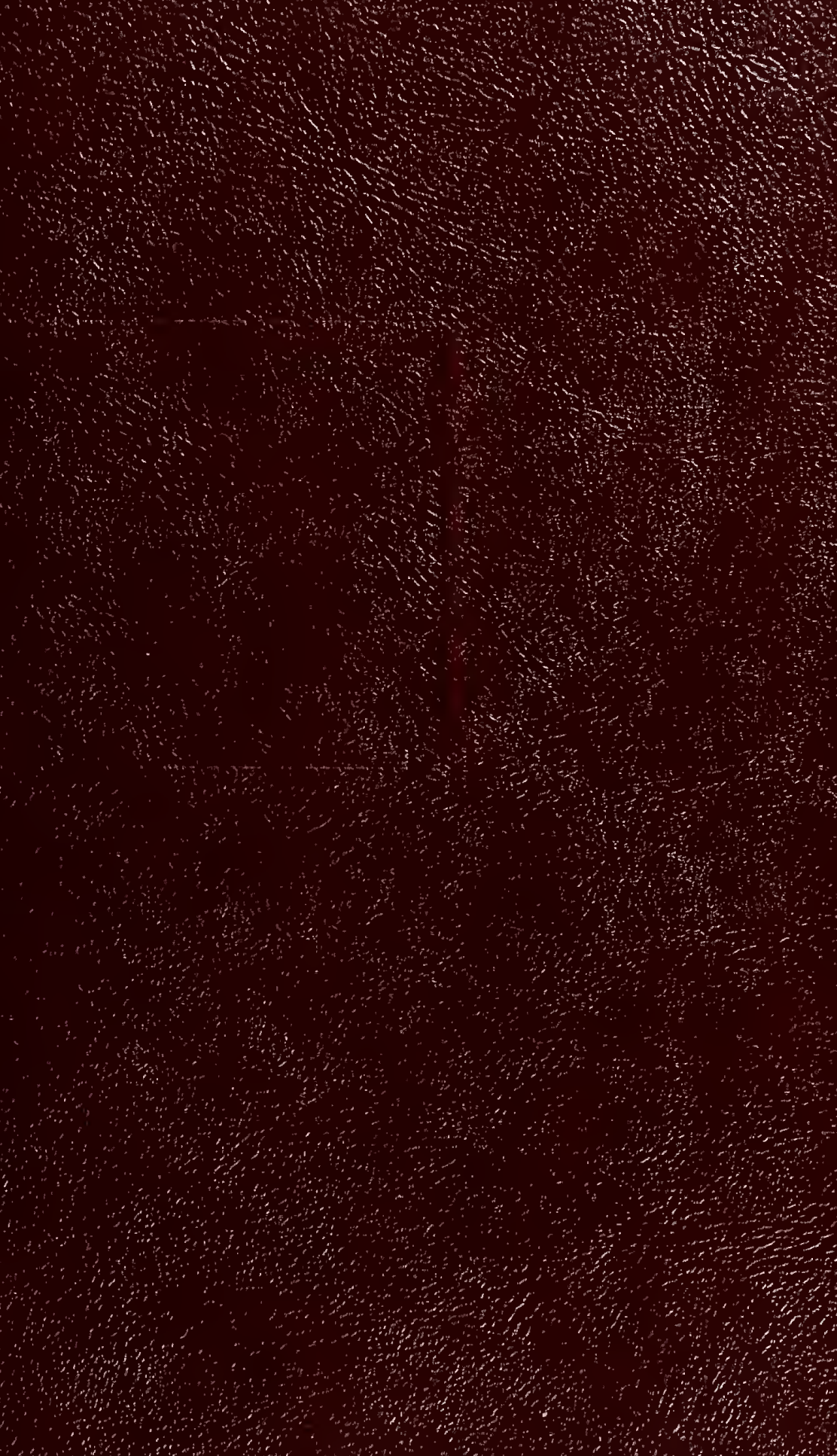




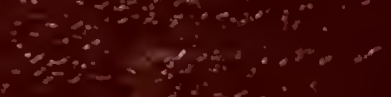


CHEMICALS REGISTERED FOR WEED PLANT CONTROL

IN MONTANA - 1977

STATE DOCUMENTS COLLECTION

MAY $P 22003$

MONTANA STAIE LIBRARY

HELENA 1515 E G Th AVE

PREPARED BY:

DAVID W. ARMSTRONG II I

BOTANIST

MONTANA DEPARTMENT OF AGRICULTURE

HELENA, MONTANA 59601 


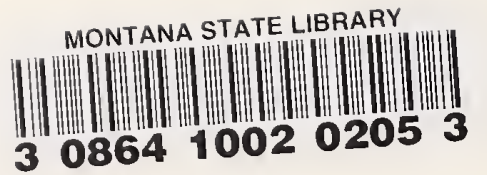




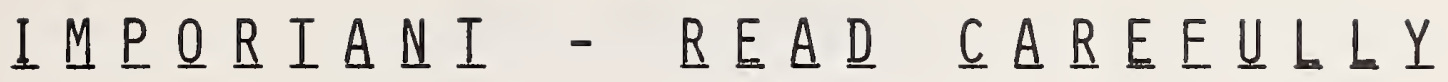

1. This publication addresses weed plant pests of alfalfa, corn, dry beans, fruit orchards, non-crop, potatoes, rangeland-pasture, small grains, and sugar beets.

2. This list is effective as of March, 1977. The deletion of registered products from this list for crop groupings covered is not intentional. The registration status of products not listed may be obtained by contacting the Montana Department of Agriculture, Pesticide Division, 1300 Cedar Street, Helena, MT 59601, phone 406-449-3730.

3. This list is intended to cover weed plant control on agricultural and non-crop areas. Home-garden type products have not been included with this list.

4. This list of registered compounds does NOT reflect nor does it replace all information and use directions listed on the label of products mentioned in this publication.

5. Dosage rates listed herein give only the range of rates listed on each label. It remains the applicator's responsibility to deternine the specific dosage rate for each pest.

6. Persons reading this list must realize that the same product sold by two companies may not have the same use directions. Therefore, one company's product may be utilized on a crop for a particular pest while another company's product may not be used on that crop for control of the same pest. Applicators are responsible for adhering to all label directions for each application of a pesticide. 
Digitized by the Internet Archive in 2016 
Format

Chemicals Registered for Weed Plant Control in Montana 1977 was written to inform Extension personnel, producers, county weed districts, and other interested parties of those herbicides registered for use on alfalfa, corn, dry beans, fruit orchards, non-crop areas, potatoes, rangeland-pasture, small grains, and sugar beets. The lists are organized alphabetically by manufacturer. Each section lists the chemical, the manufacturer, the formulation and rate, label restrictions, and weed plants controlled. Each section is preceded by an index of the weed plants controlled in each crop followed by the name and number of each chemical registered to control that pest on that particular crop. The reader can determine chemicals available for controlling a particular plant pest in a particular crop by using the index .

\section{Recommendations}

1. This list is NOT an endorsement of any product or company, nor is it a recommendation for any particular product or chemical company. It is not a recommendation for purchase or use by an applicator. All applicators are responsible for determining their choice of a pesticide according to the label specifications for pest and host.

2. Individuals making recommendations based on this list of registered products shall be responsible for their recommendations, and the Montana Department of Agriculture assumes NO responsibility.

\section{Abbreviations of Formulations}

EC - emulsifiable concentrate

G - granular

L - liquid

WP - wettable powder 



\section{Accident Prevention is Best}

1. Carefully select the right herbicide for the job.

2. Carefully and thoroughly read the label. Pay close attention to dosage rates and mixing directions. Make sure you have available all required safety equipment before you begin to mix. Use a respirator and related equipment when mixing or handling highly hazardous pesticides.

3. Handle pesticide containers carefully. Cut bags with a knife rather than tearing--dusts and powders will sometimes billow up out of the bag.

4. Never work alone when handling highly hazardous chemicals.

5. Measure quantities of chemicals carefully using clean equipment. Thoroughly clean all mixing equipment after use.

6. Clean up pesticide spills immediately. Avoid exposure of chemical to skin. When chemical does contact skin, wash it off immediately with soap and water.

7. Never siphon a pesticide from a container by mouth.

8. When filling a spray tank, do not allow delivery hose to fall below water level to avoid back siphoning.

9. Always keep a clean label with you at all times in case of accident.

10. When handling highly hazardous chemicals, especially on a regular basis, notify your physician of the chemical you will be using.

\section{IN CASE OF POISONING}

\section{Watch for These Symptoms}

1. Headache

2. Dizziness

3. Blurred vision

4. Weakness
5. Nausea

6. Cramps

7. Diarrhea

8. Discomfort in the chest

In most cases of occupational pesticide poisoning, the symptoms may not manifest themselves for several hours after exposure. 


\title{
First Aid
}

1. If breathing has ceased, apply artificial respiration immediately. Never try to give anything by mouth to an unconscious patient.

2. Get the victim to a physician or hospital as soon as possible. If the pesticide involved is known, take a label or container with you.

3. If the pesticide has been swallowed and the patient has not vomited, induce vomiting by giving a tablespoon of salt in one half glass warm water. Induce vomiting in child patients by stimulating back of throat with a blunt instrument. Keep head inverted.

4. In case of large chemical spill on the skin, remove all clothing and drench skin in generous amounts of soap and water.

5. If poison has gone into eyes, flush them immediately with large amounts of water.

6. Have patient lie down and keep them calm and warm.

\section{Poison Control Centers}

A nationwide network of Poison Control Centers is available for assistance and treatment of various poisoning situations. If a poisoning involves a pesticide with which your physician is unfamilar, he can contact the nearest Poison Control Center by phone for advice in treatment. There are currently two Poison Control Centers in Montana:

$\frac{\text { Great Falls, MT } 59401}{\text { Poison Control Center }}$

Deaconess Hospital (406) 761-1200

\author{
Billings, MT \\ Poison Control Center \\ Deaconess Hospital (406) 259-5551 \\ 28139 th Avenue North
}


TABLE I

USEFUL CONVERSION TABLES

\section{Fluid Measure}

\section{Fluid Ounces}

$\begin{array}{llll}1 / 6 & =1 \text { teaspoon (tsp) } & \\ 1 / 2 & =1 \text { tablespoon (tbls) } & =3 \text { teaspoons } \\ 1 & =2 \text { tablespoons } & =1 / 8 \text { cup } \\ 8 & =1 \text { cup } & =1 / 2 \mathrm{pint} \\ 16 & =2 \text { cups } & =1 \text { pint } \\ 32 & =4 \text { cups } & =1 \text { quart } \\ 28 & =16 \text { cups } & =1 \text { gallon }\end{array}$


TABLE II

APPROXIMATE RATES OF APPLICATION EQUIVALENTS

(U.S. Measures)

1 ounce per square foot $=2,722.5$ pounds per acre

1 ounce per square yard $=302.5$ pounds per acre

1 ounce per 100 square feet $=27.2$ pounds per acre

1 pound per 100 square feet $=435.56$ pounds per acre

1 pound per 1,000 square feet $=43.56$ pounds per acre

1 pound per acre $=1$ ounce per 2,733 square feet $(0.37 \mathrm{oz} . / 1,000 \mathrm{sq}$. ft. $)=$ 4.5 grams per gallon $=0.0104 \mathrm{gm}$. per $\mathrm{sq} . \mathrm{ft} .=1.12$ kilograms per hectare)

100 pounds per acre $=2.5$ pounds per 1,000 square feet $=1.04$ gm. per sq. ft.

5 gallons per acre $=1$ pint per 1,000 square feet $=0.43 \mathrm{~m} 1$. per sq. ft.

100 gallons per acre $=2.5$ gallons per 1,000 square feet $=1$ quart per 100 square feet $=935$ 1iters per hectare

1 quart per 100 gallons (approximate) $=10 \mathrm{ml}$. per gallon

1 pound per gallon $=120$ grams per liter

1 kilogram per hectare $=0.89$ pound per acre 
TABLE III

CONVERSION TABLE FOR USE OF MATERIALS ON SMALL AREAS

\begin{tabular}{|c|c|c|c|}
\hline Rate Per Acre & Rate Per 1,000 Square Feet & Rate Per 100 & Squa: \\
\hline & Dry Materials & & \\
\hline 1 pounds & 0.35 ounces & 0.25 & tsp. \\
\hline 2 & 0.7 & 0.50 & tsp. \\
\hline 3 & 1.1 & 0.75 & tsp. \\
\hline 4 & 1.4 & 1.00 & tsp. \\
\hline 5 & 1.8 & 1.25 & tsp. \\
\hline 6 & 2.1 & 1.50 & tsp. \\
\hline 8 & 2.8 & 1.55 & tsp. \\
\hline 10 & 3.7 & 2.00 & tsp. \\
\hline 20 & 7.3 & 0.73 & oz. \\
\hline 40 & 14 & 1.4 & oz . \\
\hline 50 & 18 & 1.8 & oz. \\
\hline 100 & 37 & 3.7 & oz. \\
\hline 200 & 73 & 7.3 & oz. \\
\hline 300 & 110 & 11.0 & oz. \\
\hline 400 & 147 & 14.7 & Oz. \\
\hline 500 & 184 & 18.4 & oz. \\
\hline
\end{tabular}

Liquid Materials

$\begin{array}{rlr}1 \text { pt. } & 0.36 \mathrm{fl.oz.} & 0.25 \text { tsp. } \\ 1 \text { qt. } & 0.72 \text { fl.oz. } & 0.50 \text { tsp. } \\ 1 \text { gal. } & 2.9 \text { fl.oz. } & 2.00 \text { tsp. } \\ 2 \text { gal. } & 5.9 \text { fl.oz. } & 4.00 \text { tsp. } \\ 3 \text { gal. } & 8.8 \text { fl. oz. } & 2.00 \text { tbsp. } \\ 5 \text { gal. } & 14.7 \text { f1. oz. } & 3.00 \text { tbsp. } \\ 10 \text { gal. } & 29.4 \text { fl. oz. } & 6.00 \text { tbsp. } \\ 15 \text { gal. } & 2.8 \text { pts. } & 9.00 \text { tbsp. } \\ 20 \text { gal. } & 3.7 \text { pts. } & 12.00 \text { tbsp. } \\ 25 \text { gal. } & 2.3 \text { qts. } & 15.00 \text { tbsp. } \\ 50 \text { gal. } & 4.6 \text { qts. } & 1.84 \text { cups } \\ 75 \text { gal. } & 6.9 \text { qts. } & 2.76 \text { cups } \\ 00 \text { gal. } & 9.19 \text { qts. } & 3.67 \text { cups } \\ 00 \text { gal. } & 18.37 \text { qts. } & 7.35 \text { cups }\end{array}$

Dry: $1 \mathrm{lb} . / 100 \mathrm{gal}=1 \mathrm{oz} . / 6.25 \mathrm{gal}=0.5 \mathrm{oz} . / 3 \mathrm{gal} .=4.5 \mathrm{gm} . / \mathrm{gal}$

Liquid: $1 \mathrm{pt} . / 100 \mathrm{gal} .=1 \mathrm{fl} .0 \mathrm{z} . / 6.25 \mathrm{gal}=0.5 \mathrm{fl} .0 \mathrm{z} . / 3 \mathrm{gal}=1 \mathrm{tsp} . / \mathrm{gal}$.

$1 \mathrm{qt} . / 100 \mathrm{gal} .=10 \mathrm{tsp} . / 5 \mathrm{gal} .=2 \mathrm{tbsp} . / 3 \mathrm{gal} .=2 \mathrm{tsp} \cdot / \mathrm{gal}$.

$1 \mathrm{gal} . / 100 \mathrm{gal} .=0.75$ cup \& $4 \mathrm{tsp} . / 5 \mathrm{gal} .=0.5 \mathrm{cup} / 3 \mathrm{gal} .=8 \mathrm{tsp} . / \mathrm{gal}$. 


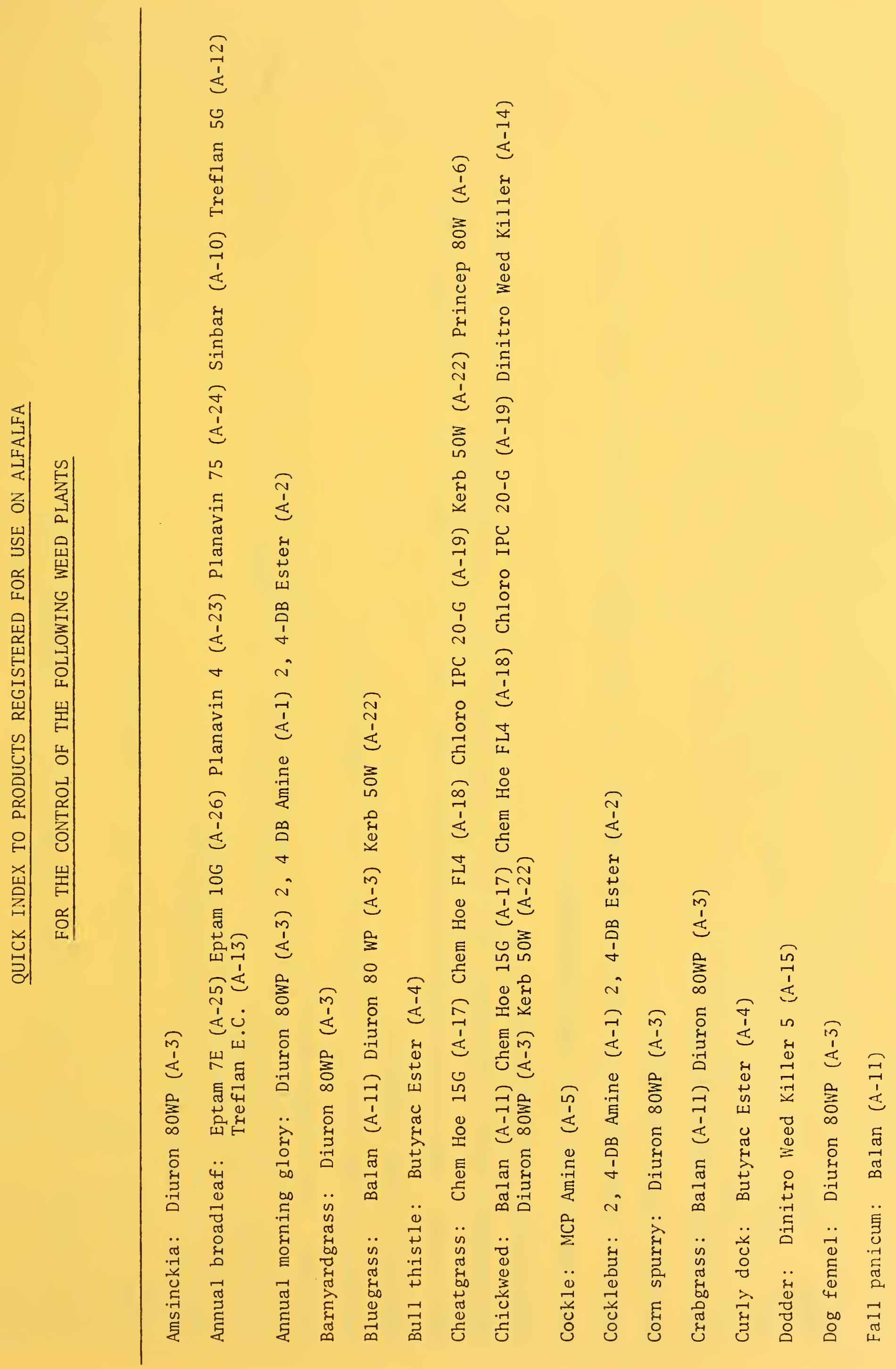




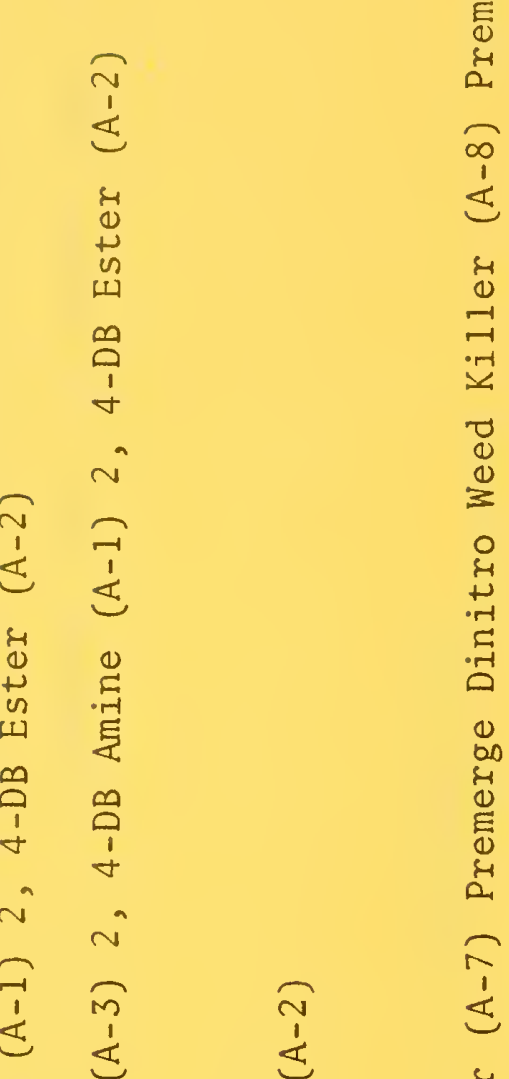

(1)

छั

$\infty$
1
$\substack{5 \\ 5}$

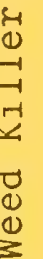

त) क ह

ह $\frac{1}{2} \quad 4$

4 告

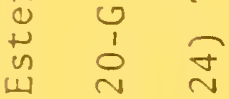

m 013

I 10

ن)

न हु

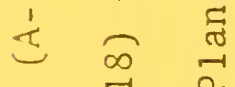

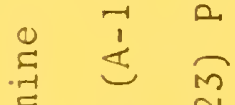

是 寸

药 出

$\begin{array}{ll}1 & 0 \\ 1 & 0\end{array}$

i E

ก ह

है $\frac{7}{1}$

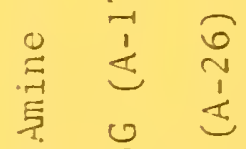

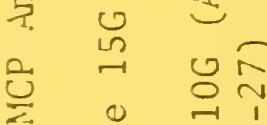

20 논

Э 이 튠

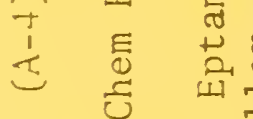

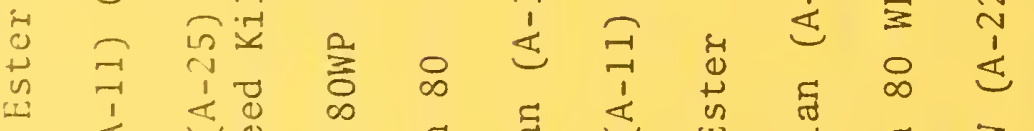

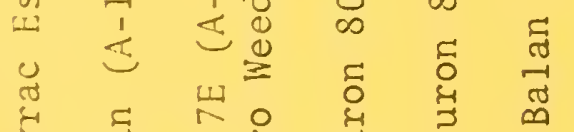

苛

$\frac{1}{5}$

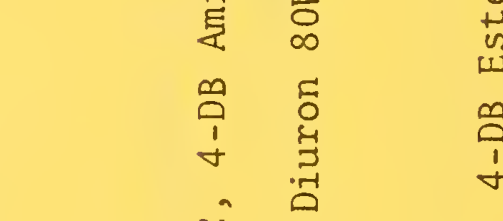

离

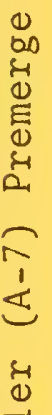

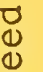

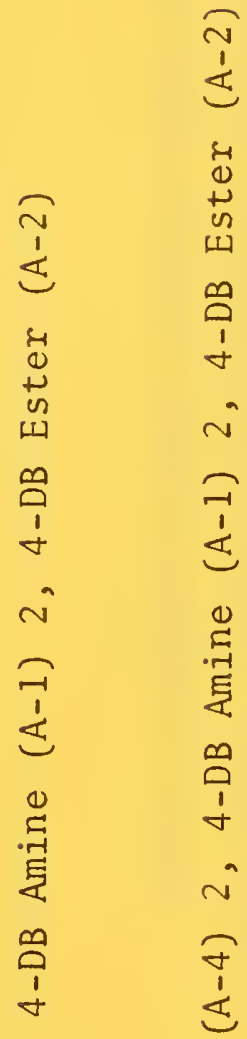

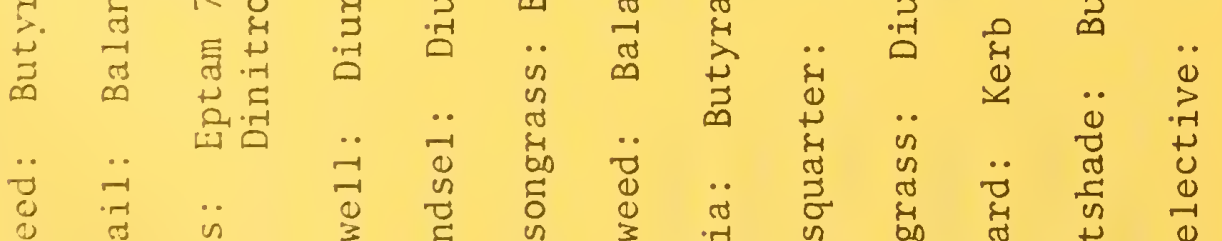

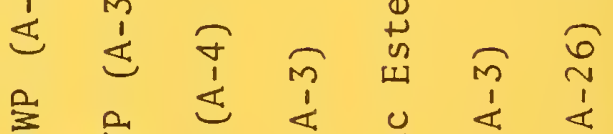
䓂

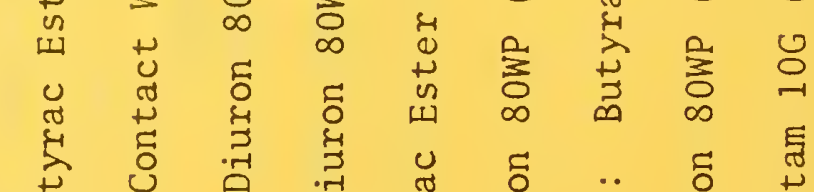




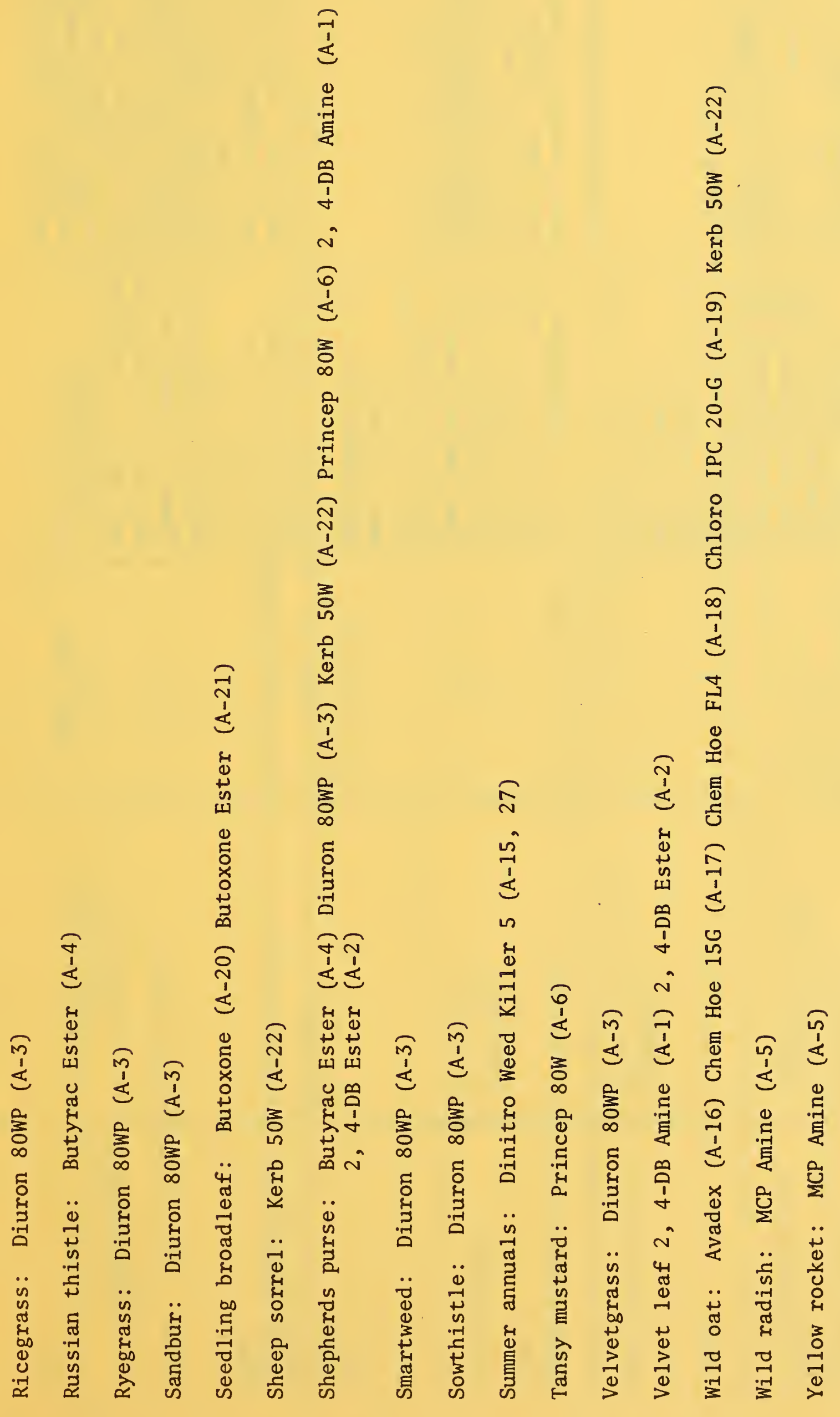




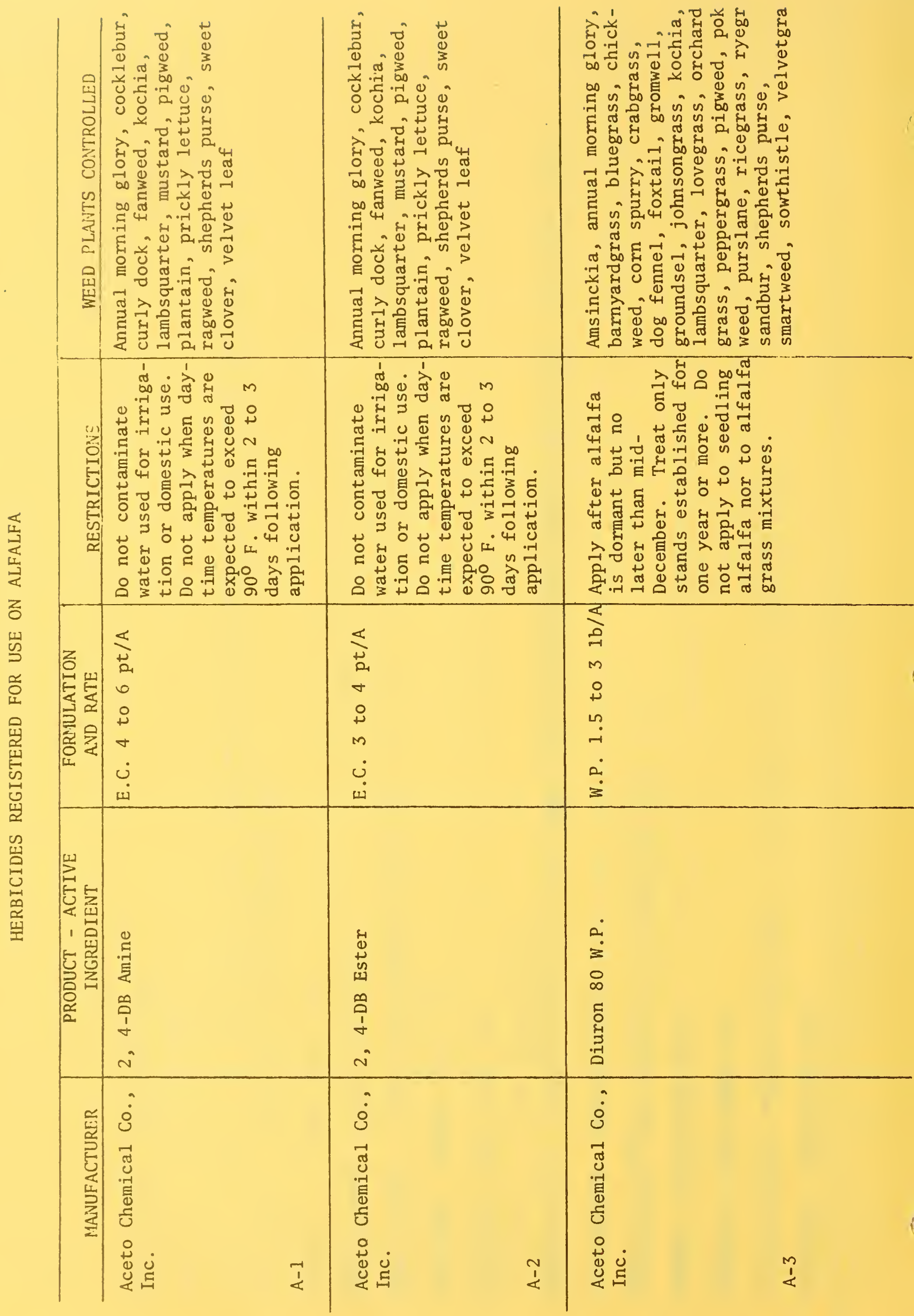




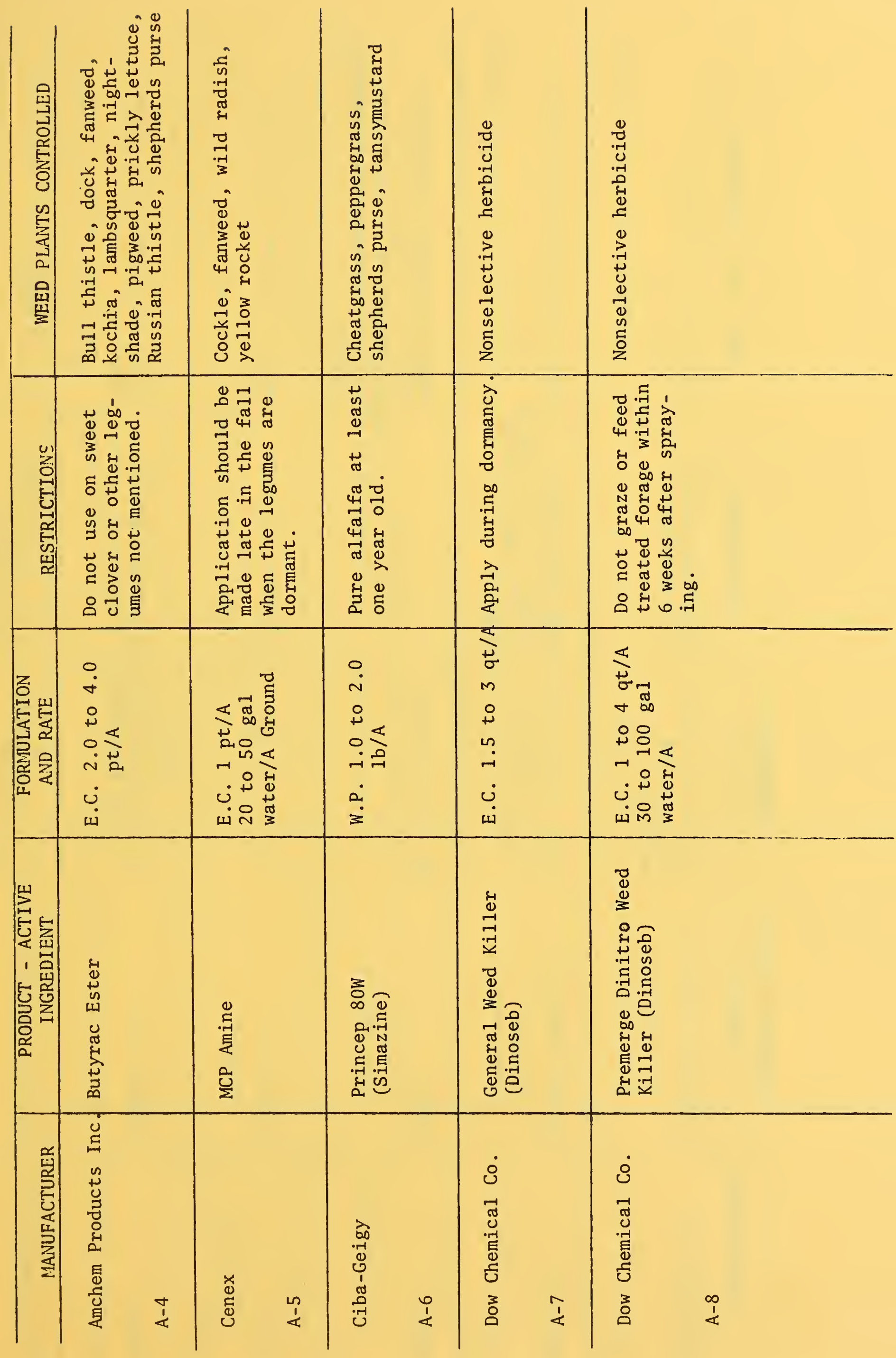




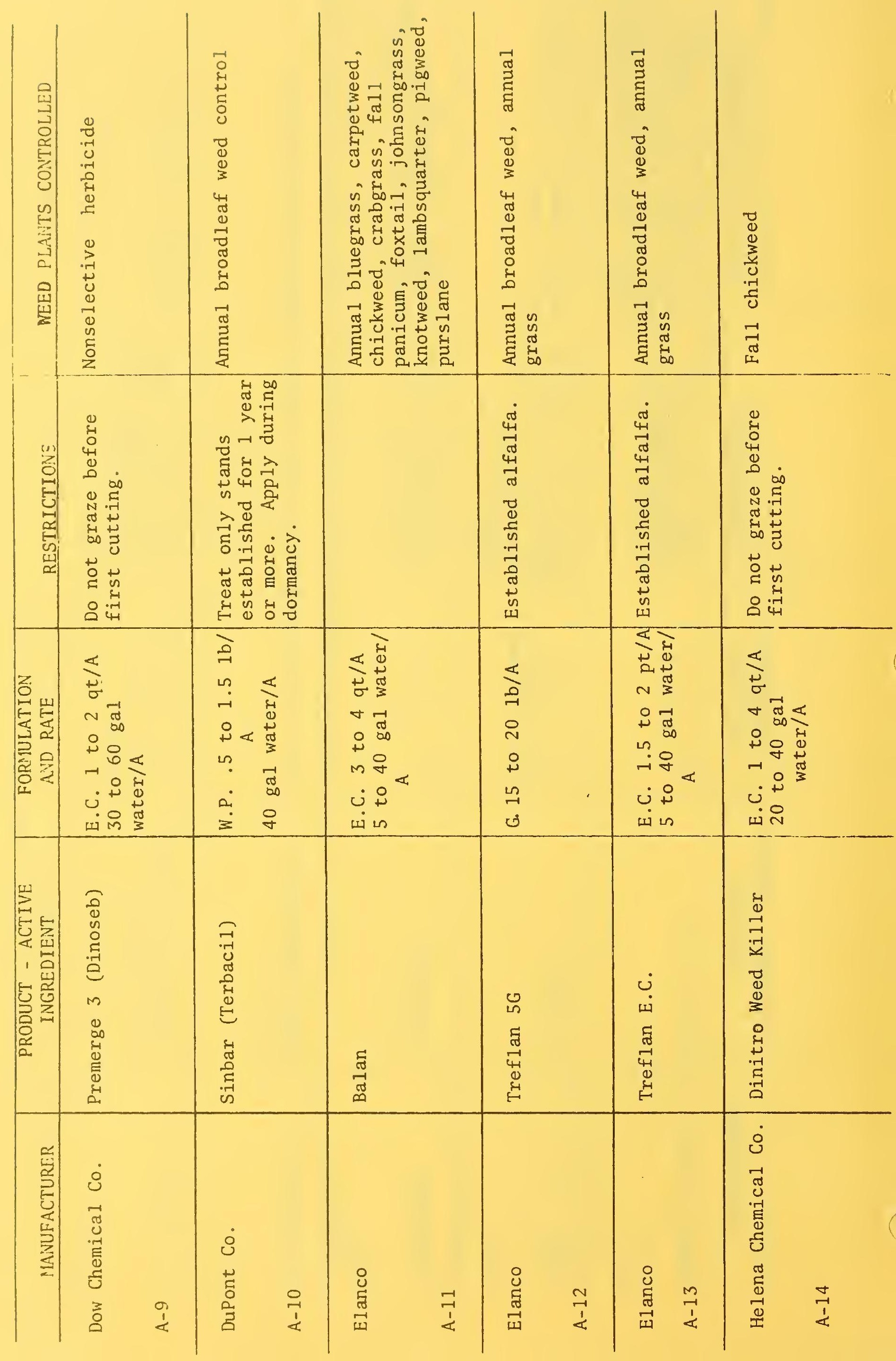




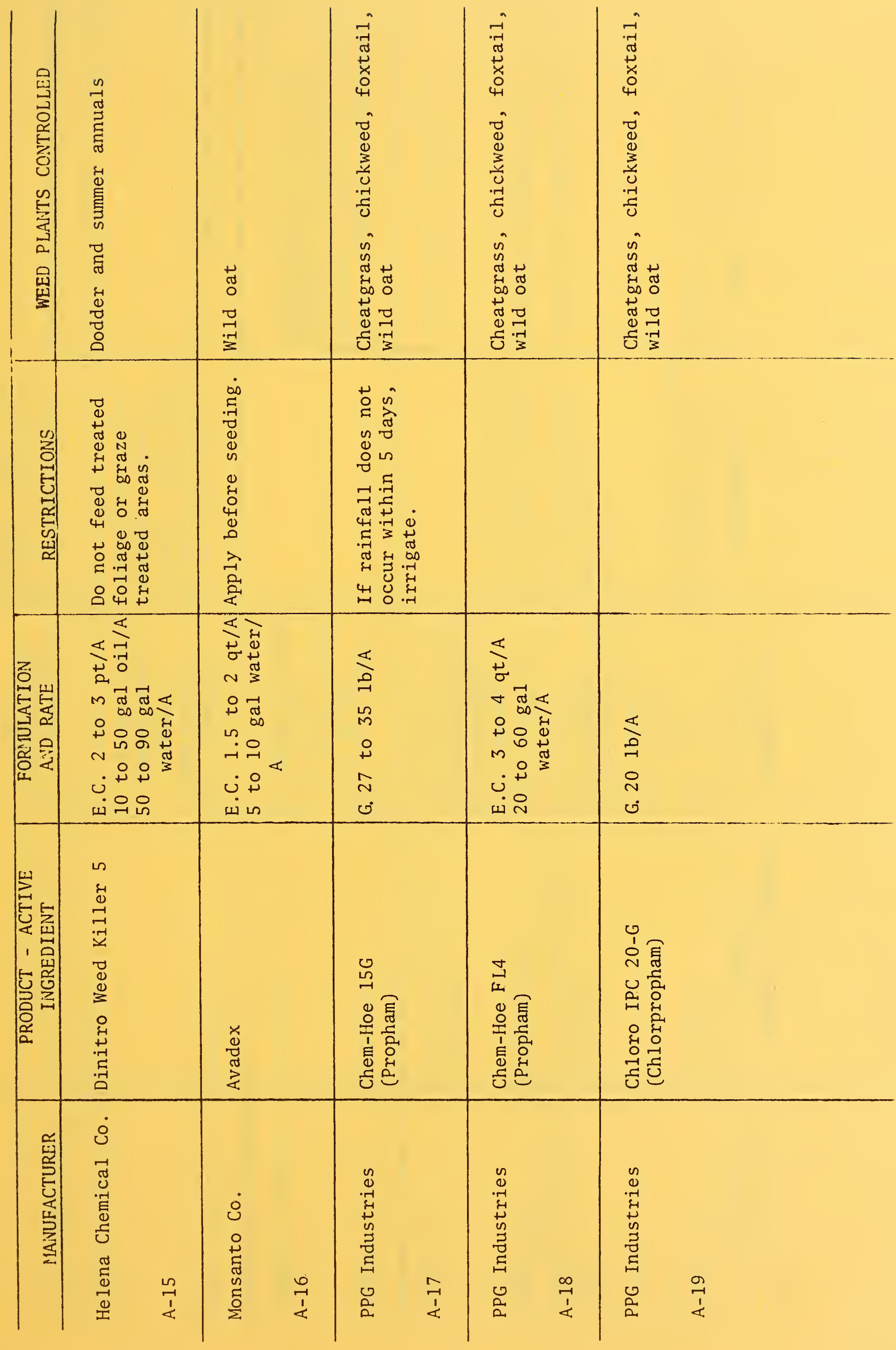




\begin{tabular}{|c|c|c|c|c|c|}
\hline 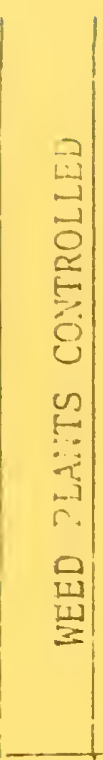 & 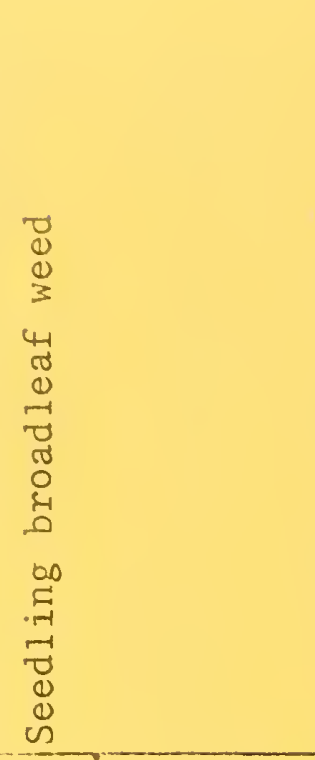 & 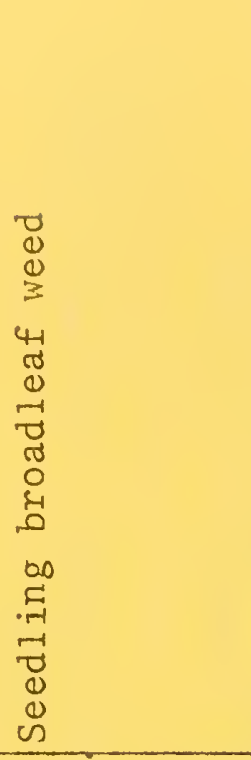 & 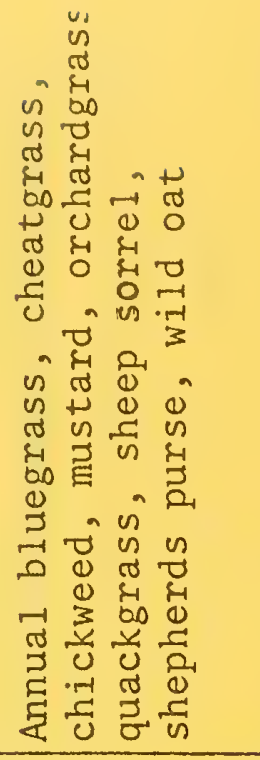 & 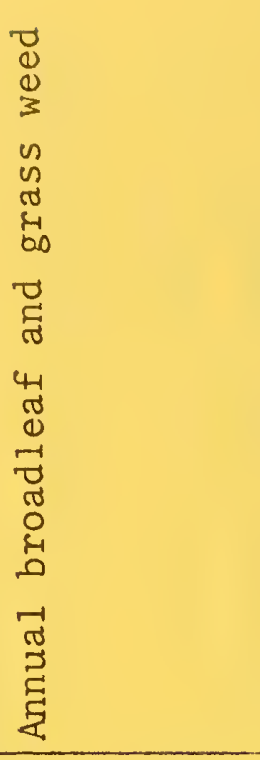 & 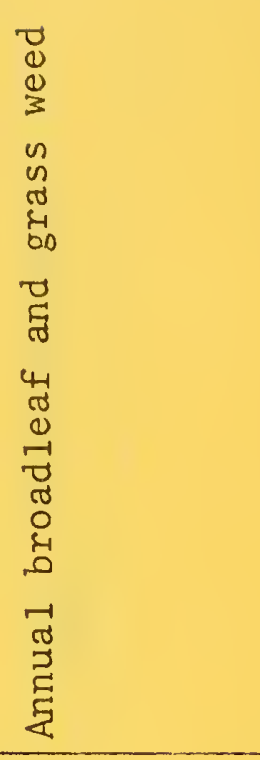 \\
\hline 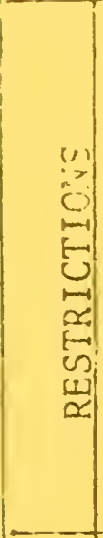 & 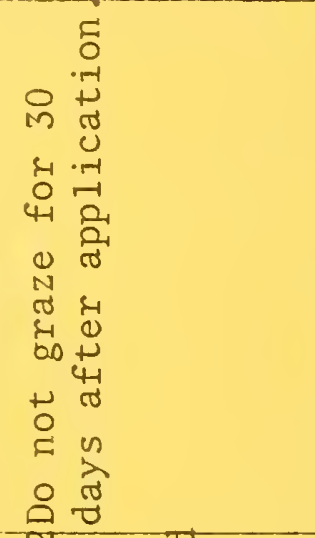 & 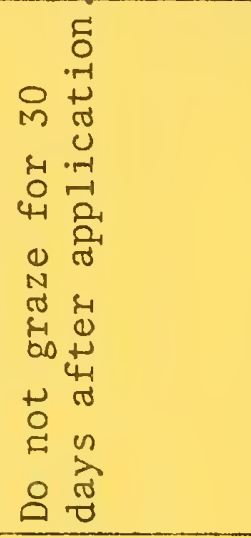 & & 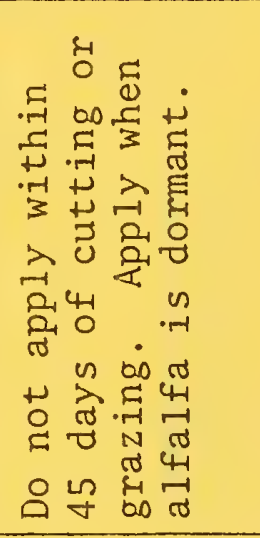 & 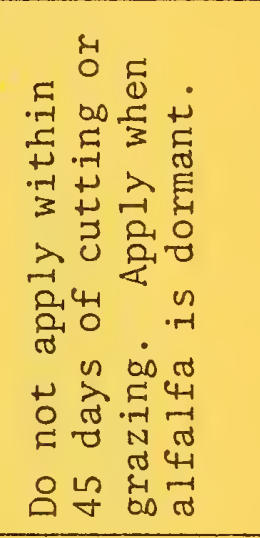 \\
\hline 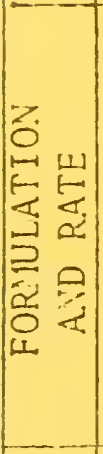 & 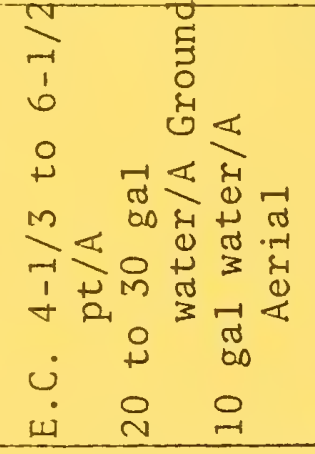 & 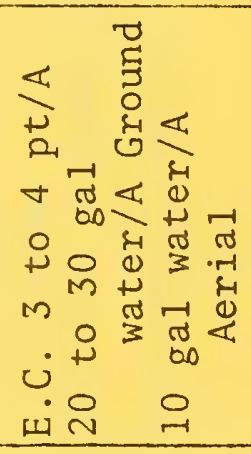 & 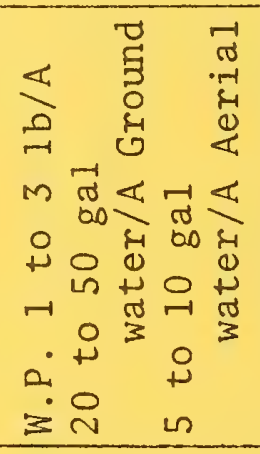 & 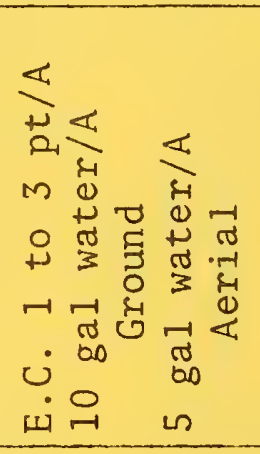 & 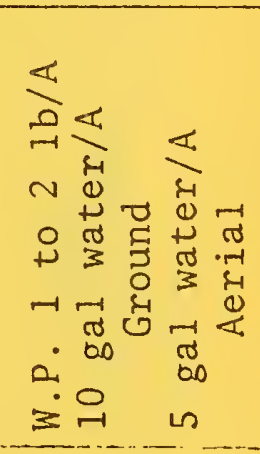 \\
\hline 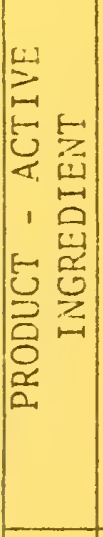 & 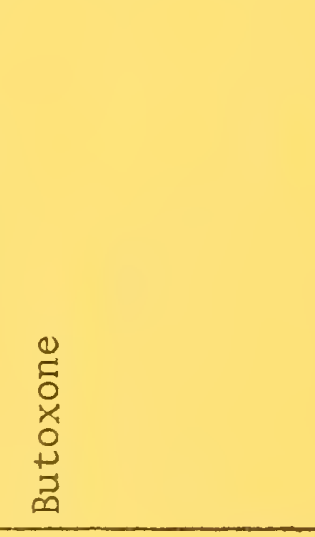 & 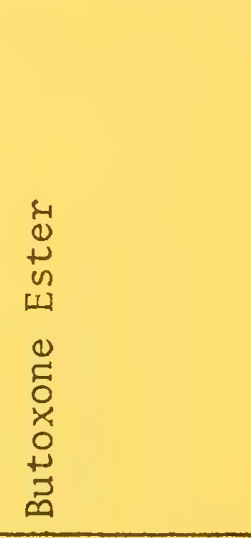 & 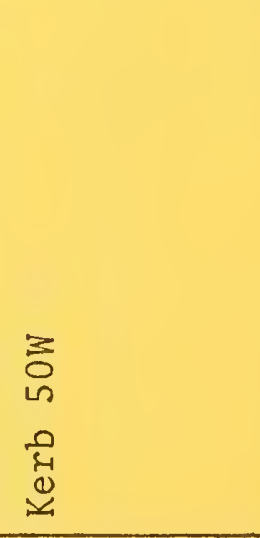 & 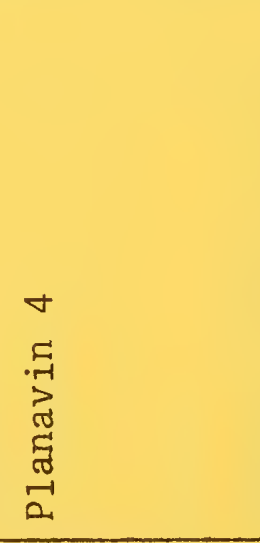 & 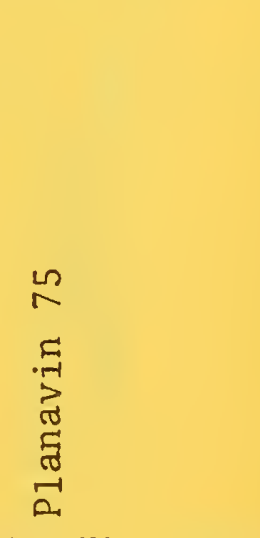 \\
\hline 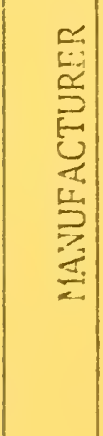 & 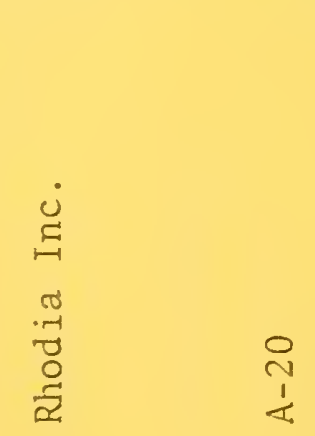 & 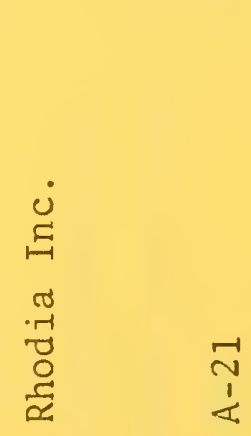 & 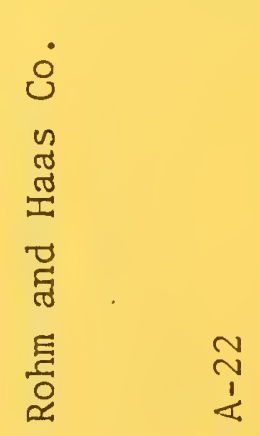 & 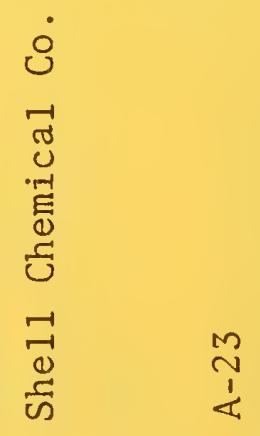 & 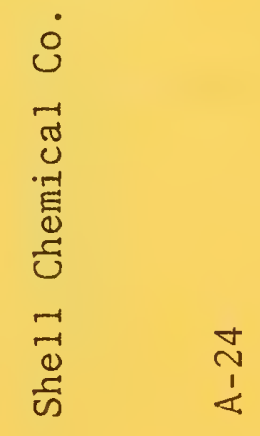 \\
\hline
\end{tabular}




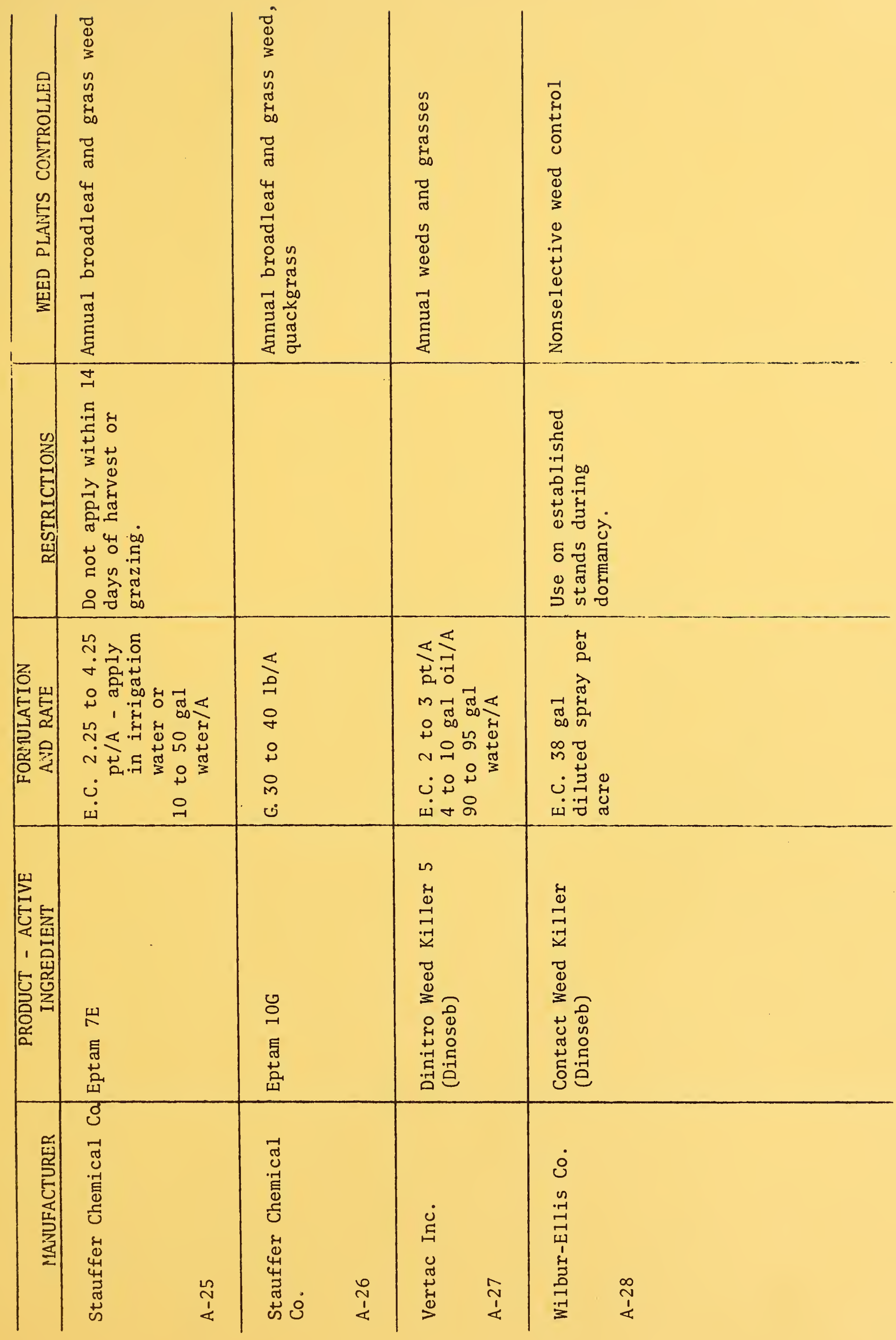






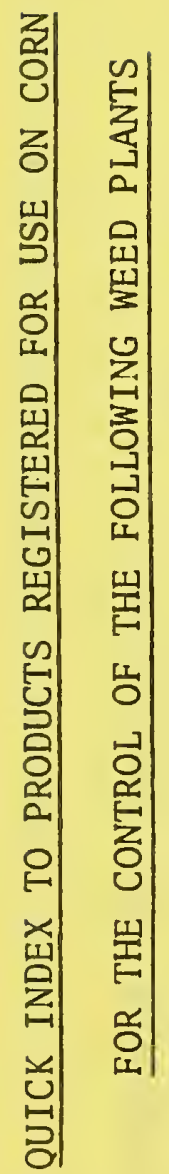

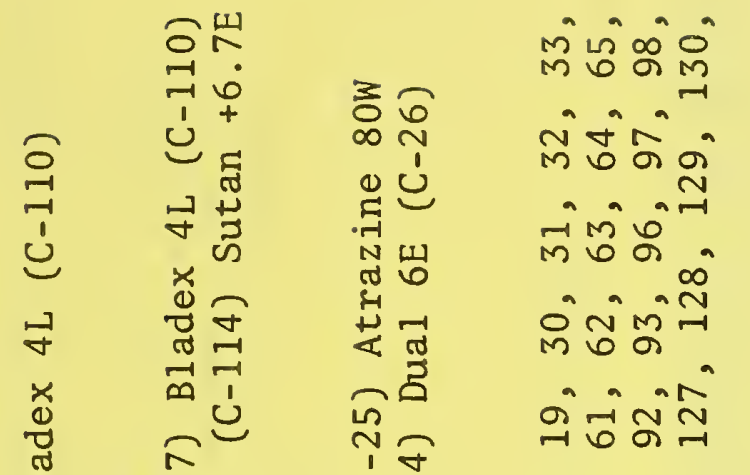

ซ

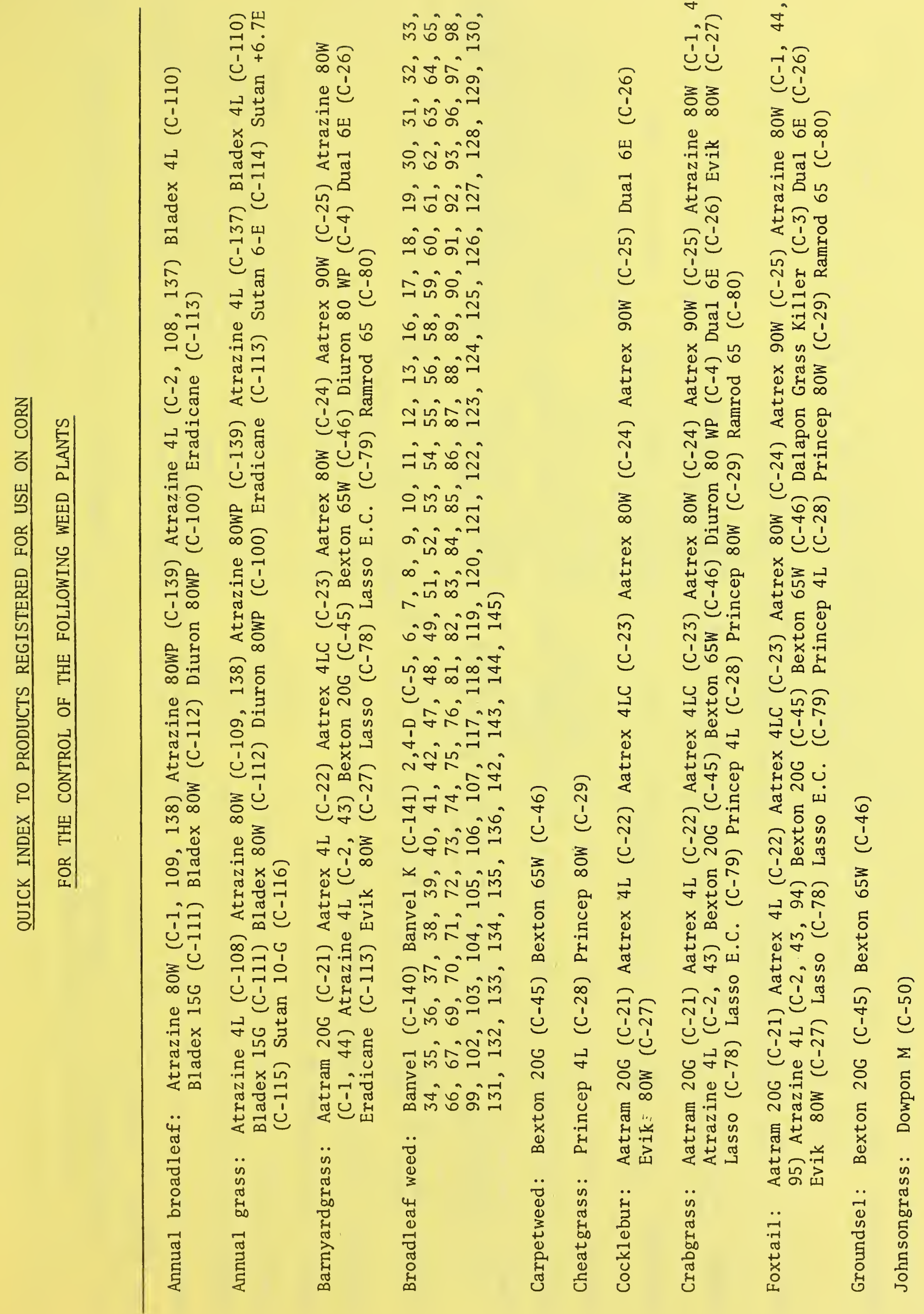




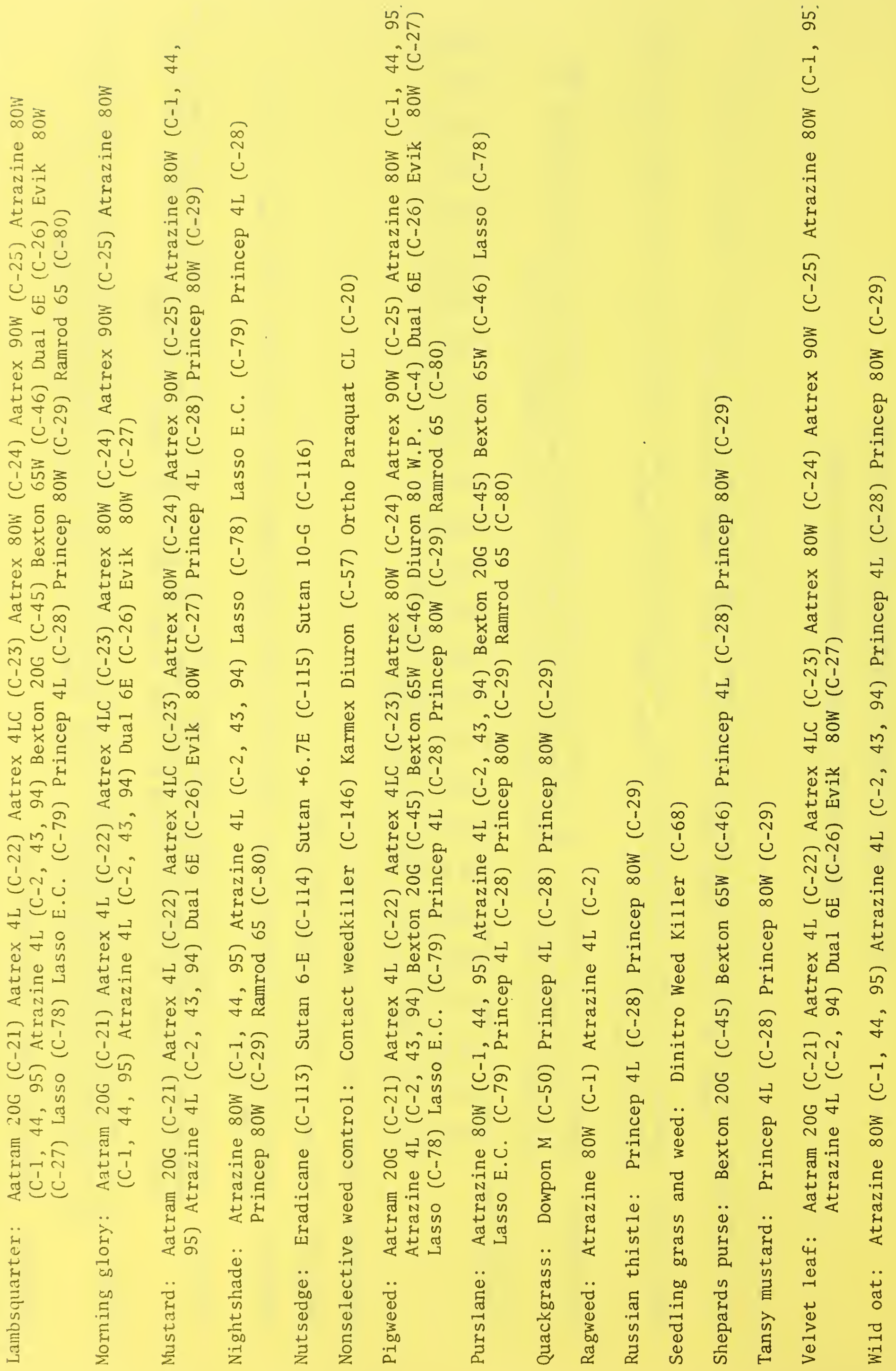




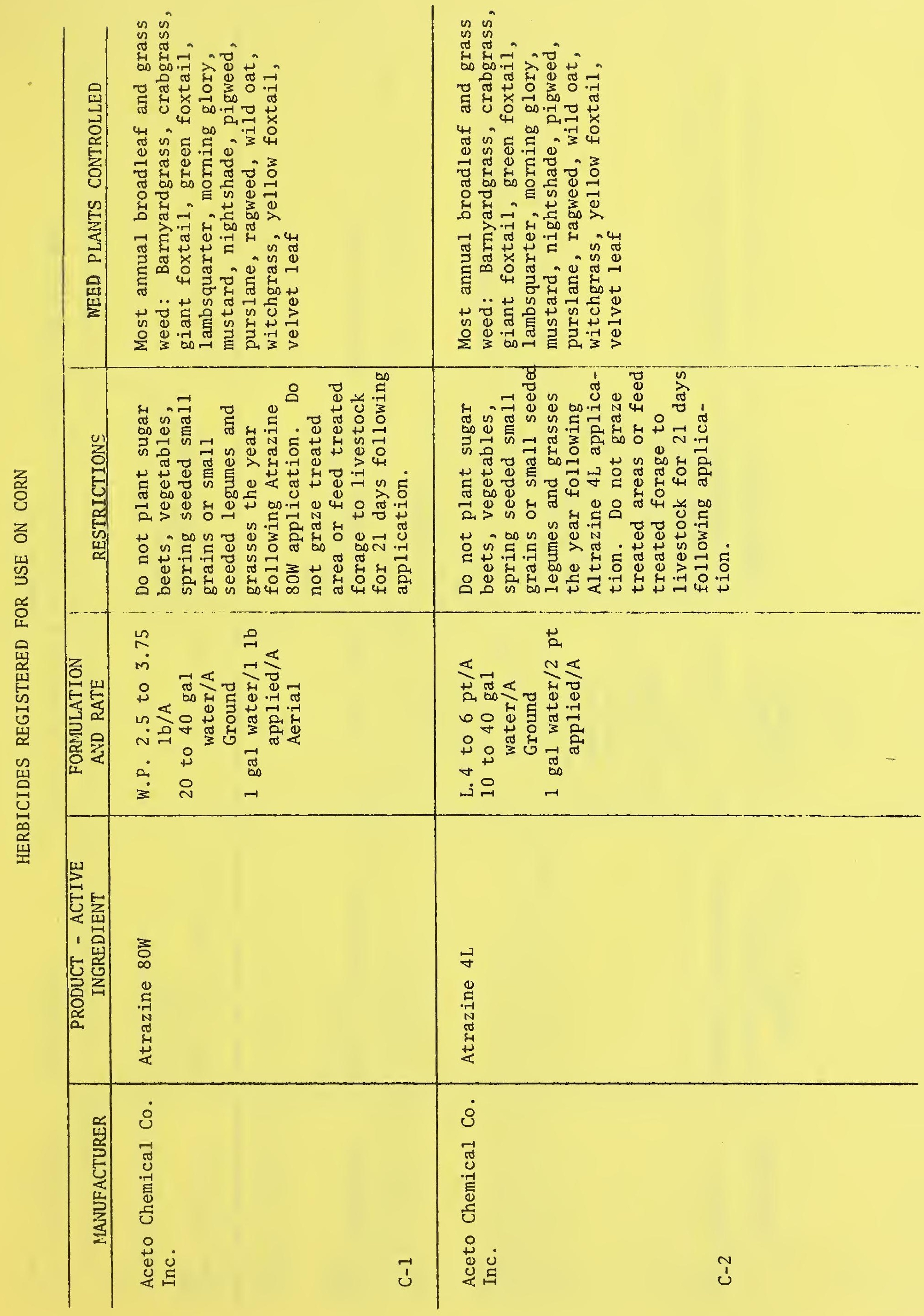




\begin{tabular}{|c|c|c|c|c|c|}
\hline 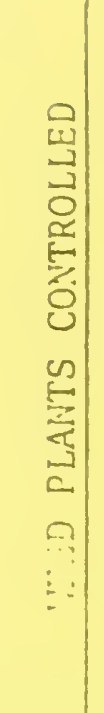 & 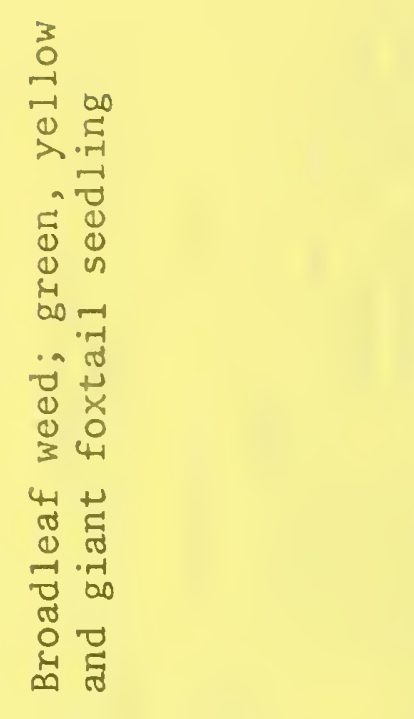 & 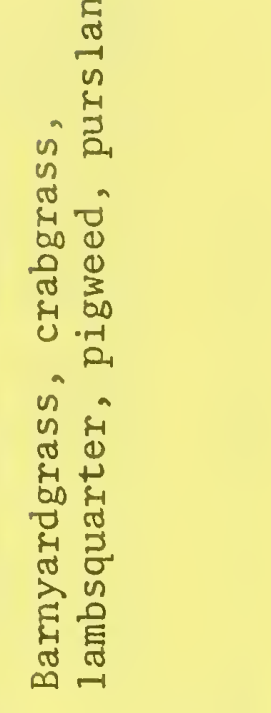 & 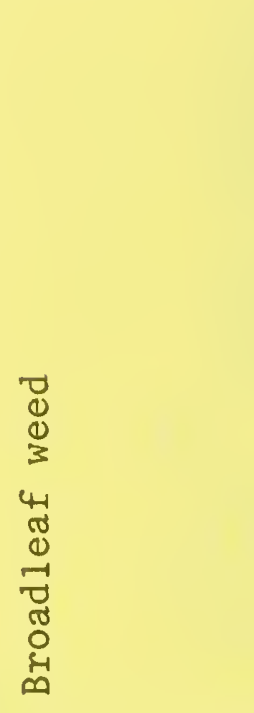 & 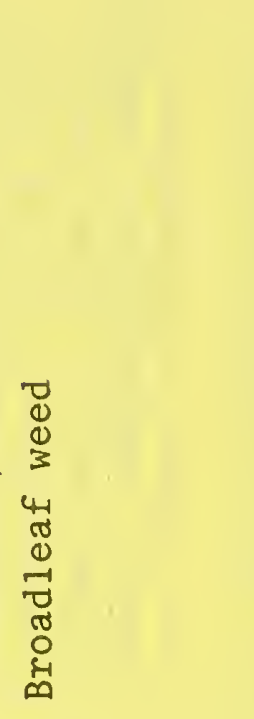 & 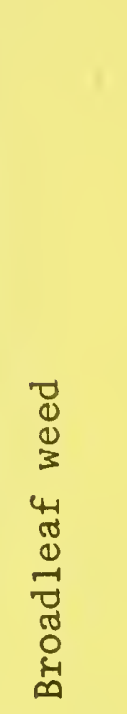 \\
\hline 党: & 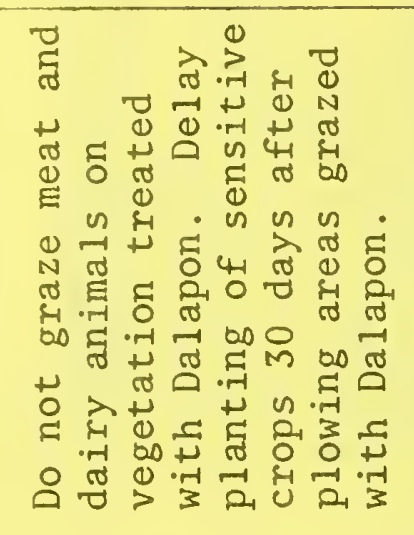 & 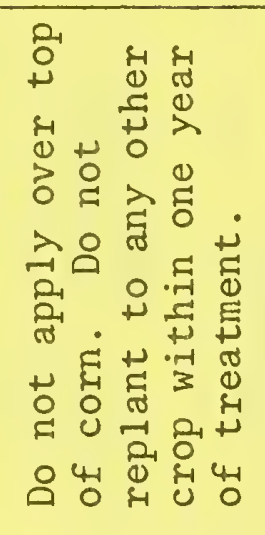 & 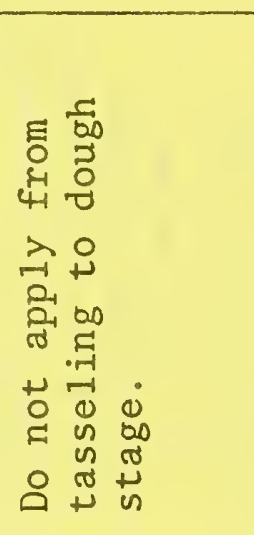 & 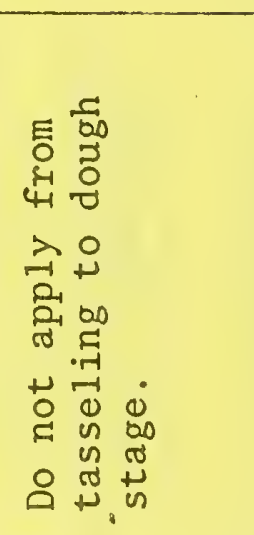 & 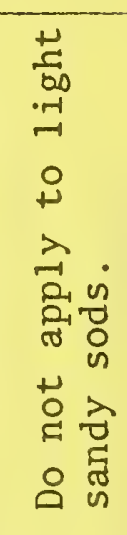 \\
\hline 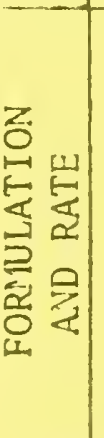 & 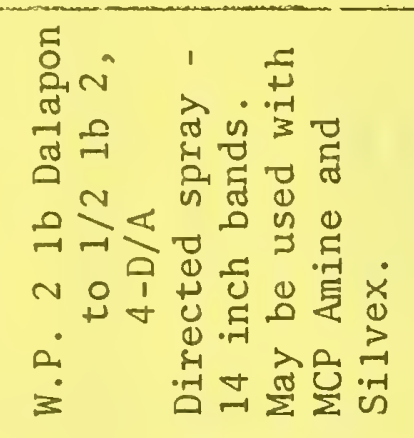 & 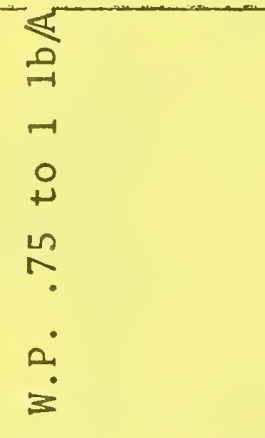 & 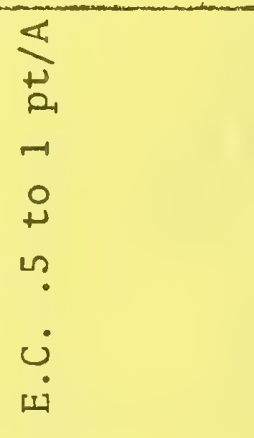 & 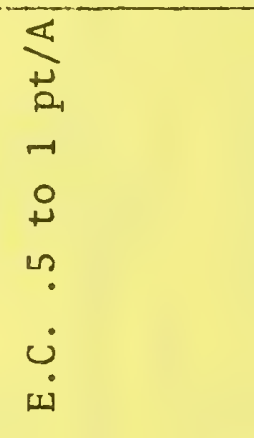 & 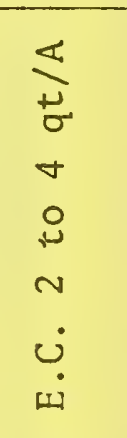 \\
\hline 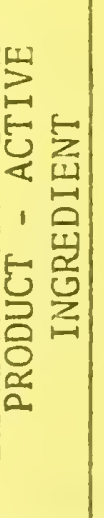 & 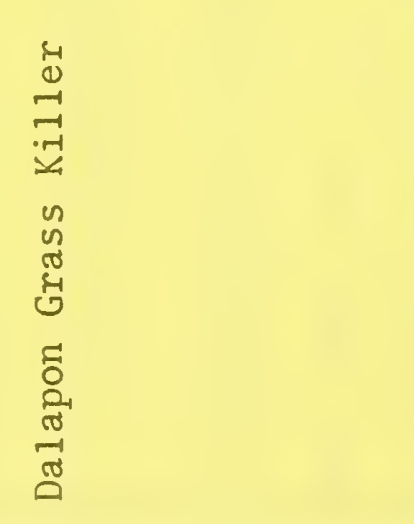 & 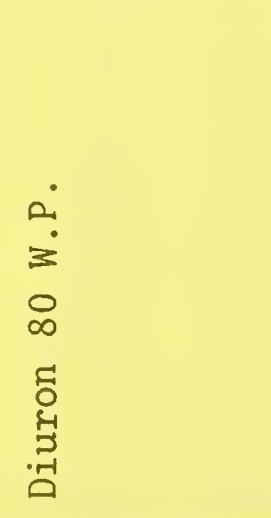 & 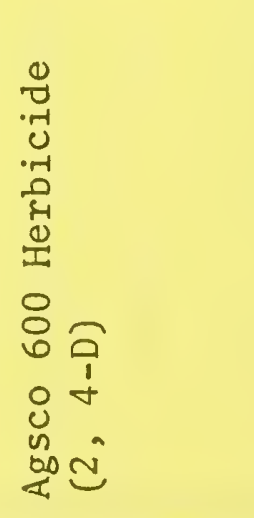 & 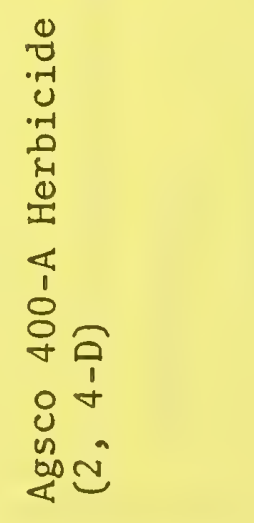 & 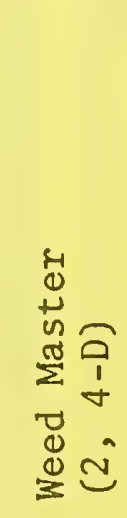 \\
\hline 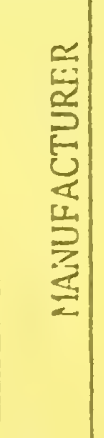 & 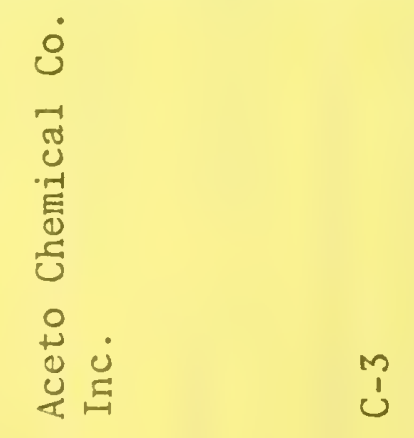 & 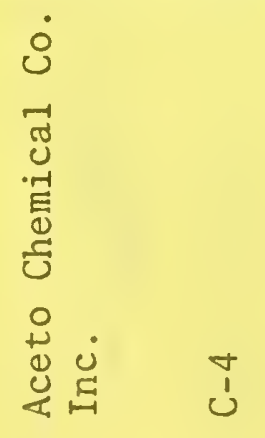 & 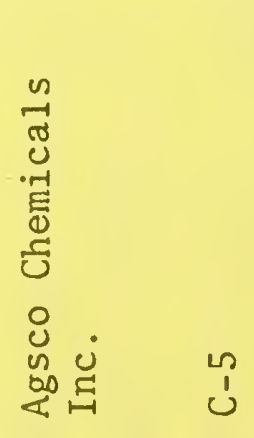 & 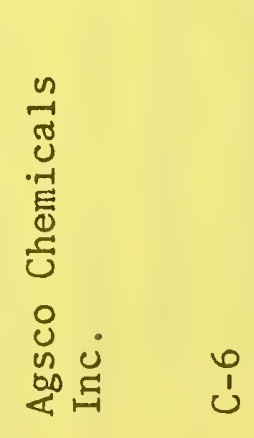 & 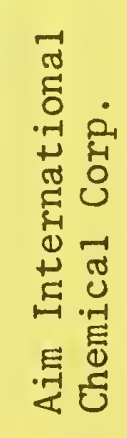 \\
\hline
\end{tabular}




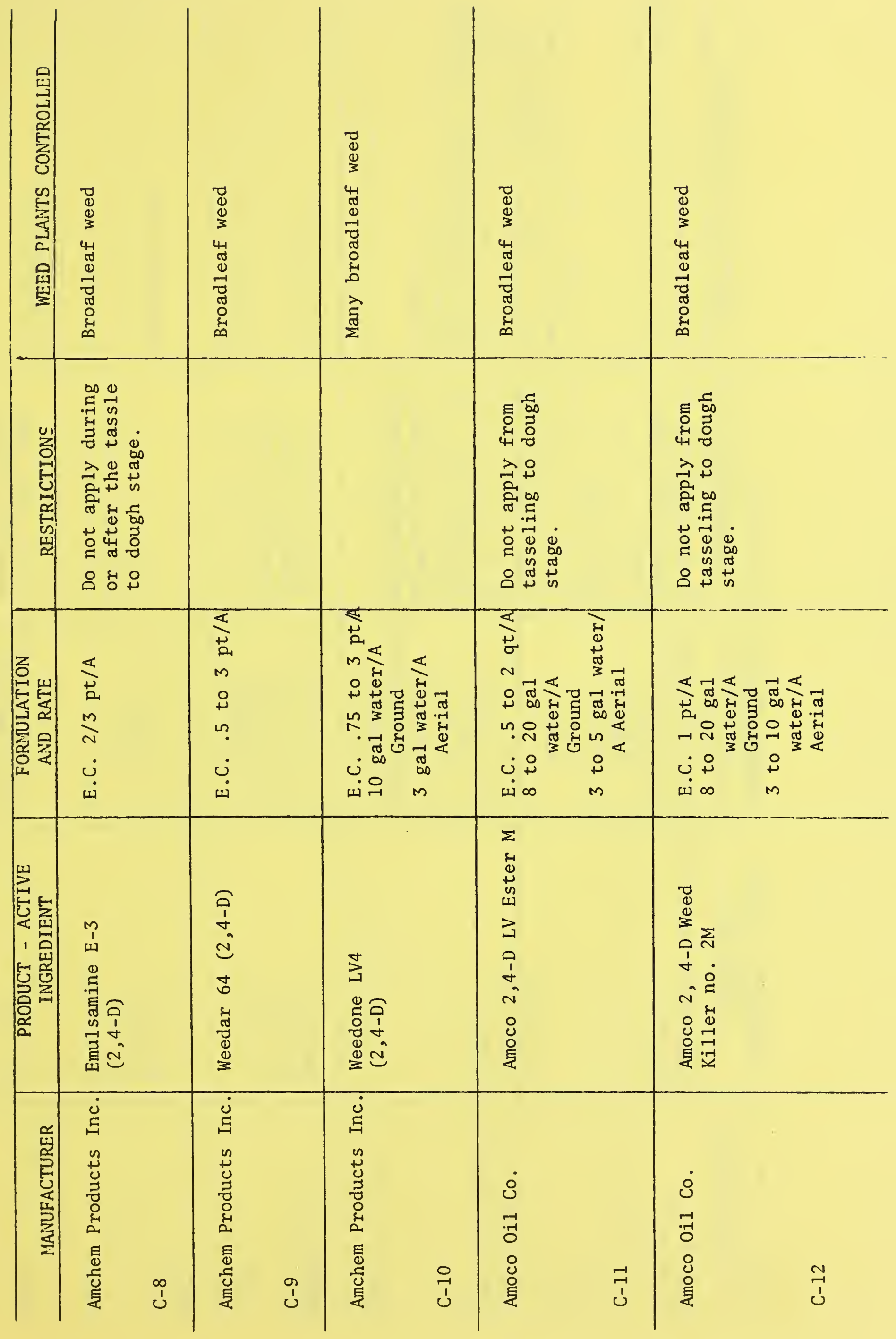




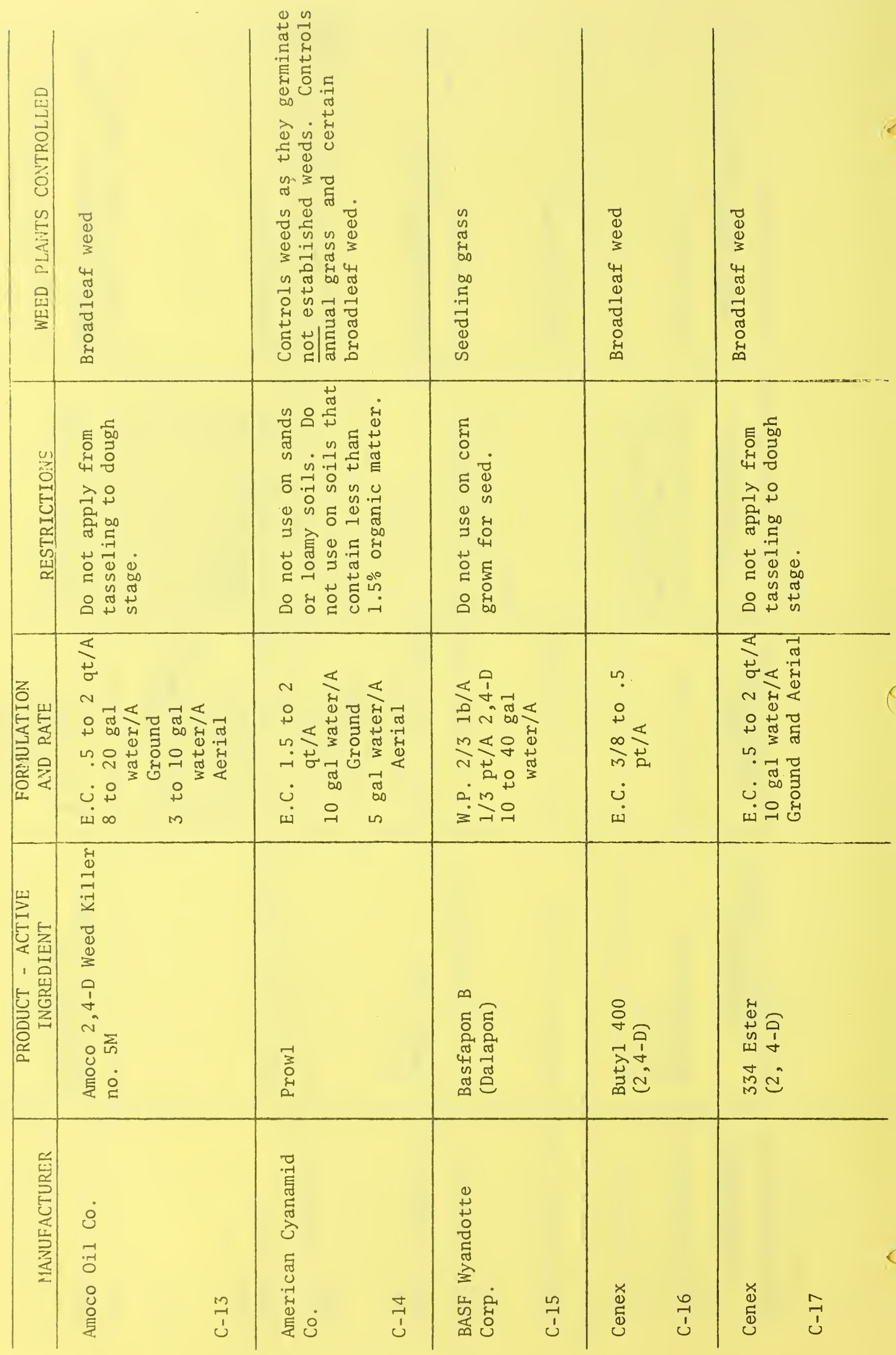




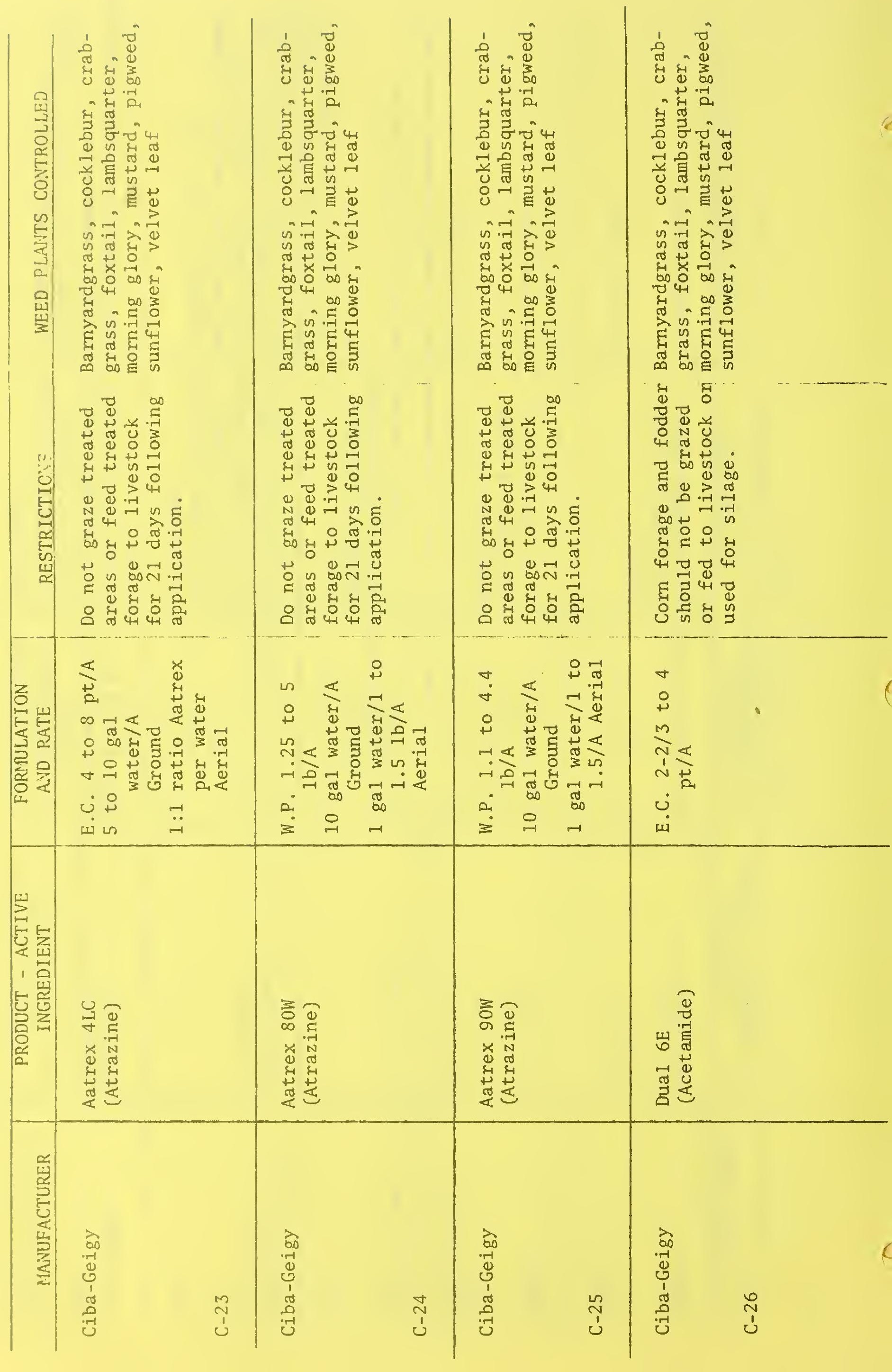




\begin{tabular}{|c|c|c|c|c|c|}
\hline 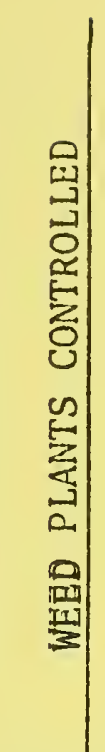 & 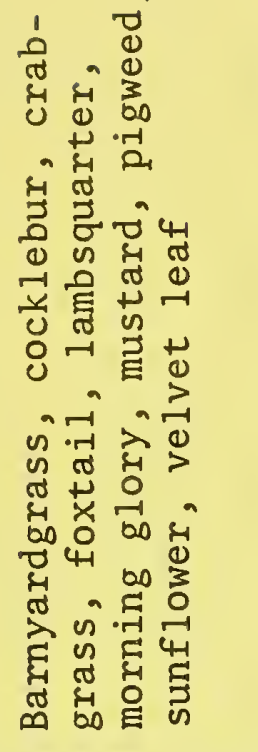 & 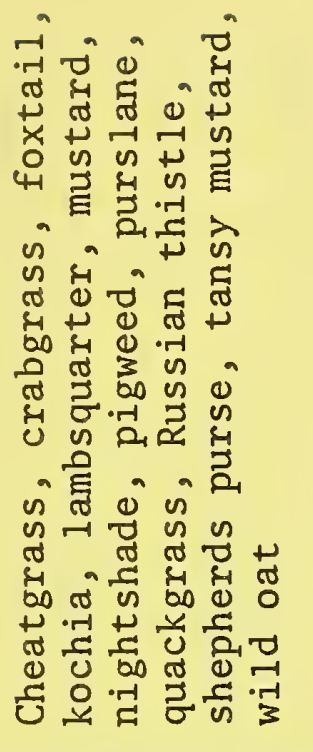 & 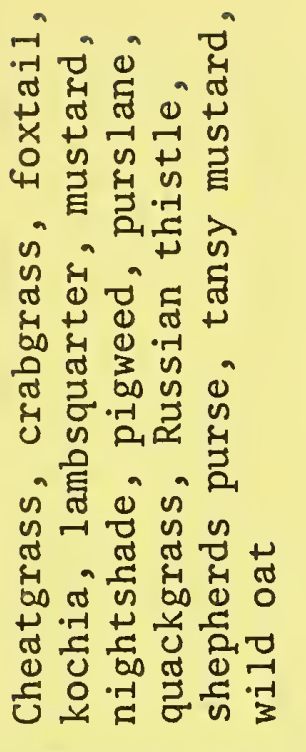 & 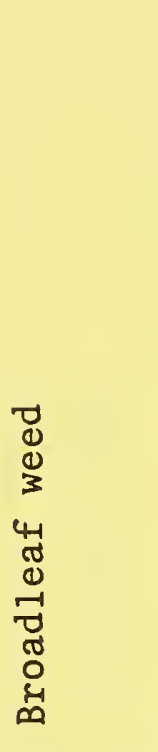 & \\
\hline 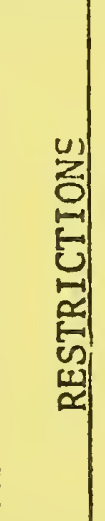 & 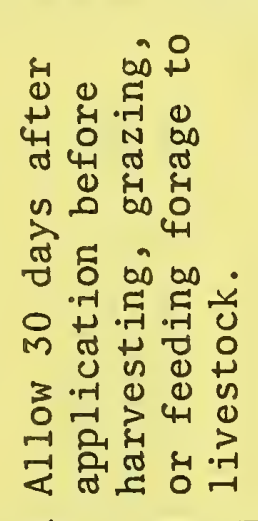 & 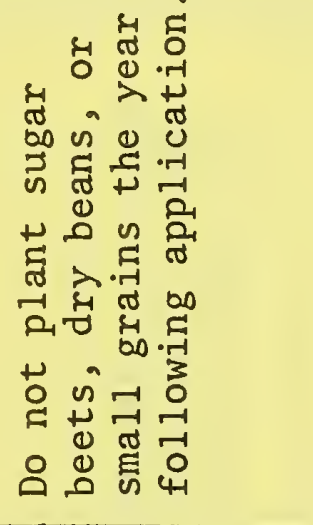 & 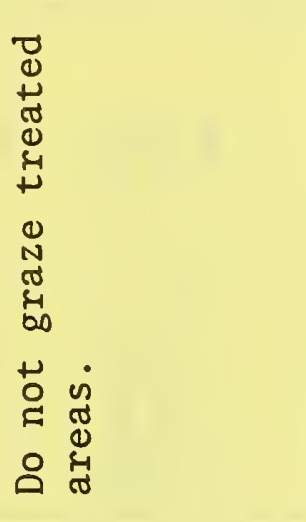 & 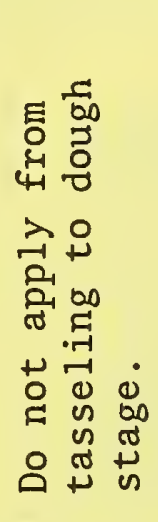 & \\
\hline 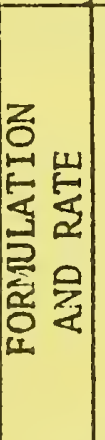 & 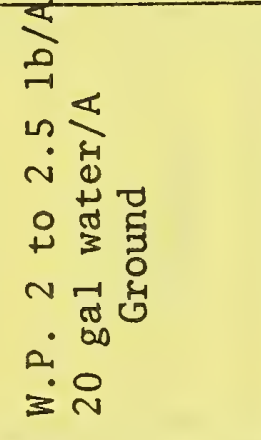 & 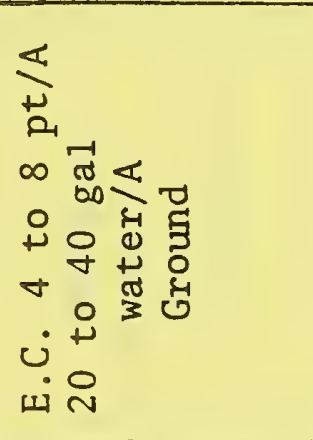 & 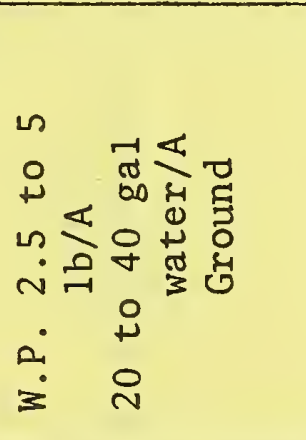 & 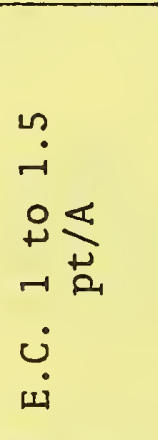 & \\
\hline 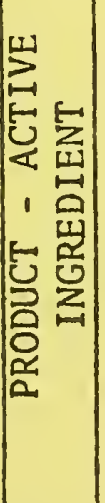 & 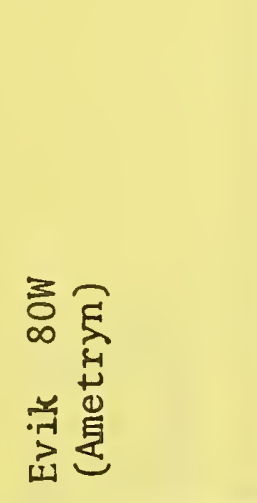 & 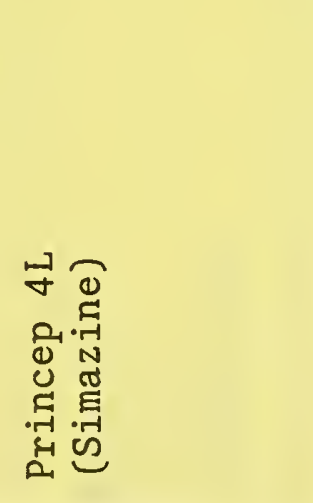 & 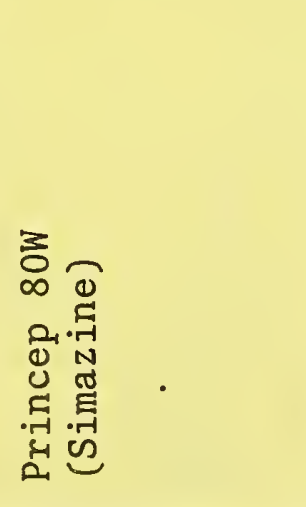 & 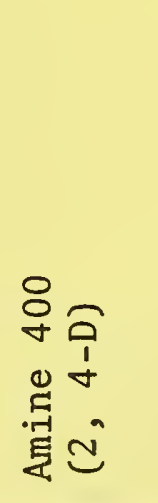 & \\
\hline 点 & 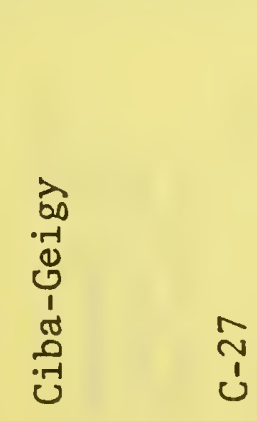 & $\begin{array}{l}\text {. } \\
.0 \\
0 \\
0 \\
1 \\
0 \\
0 \\
0 \\
0 \\
0\end{array}$ & 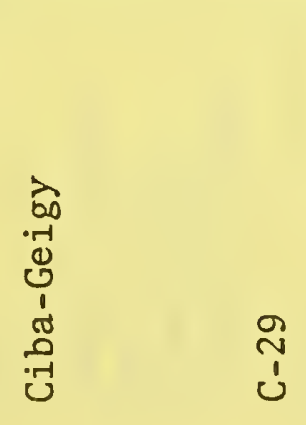 & 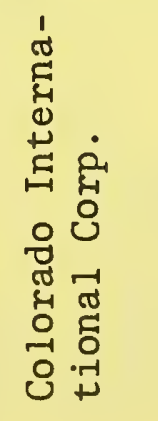 & $\begin{array}{l}0 \\
\text { m } \\
\dot{1}\end{array}$ \\
\hline
\end{tabular}




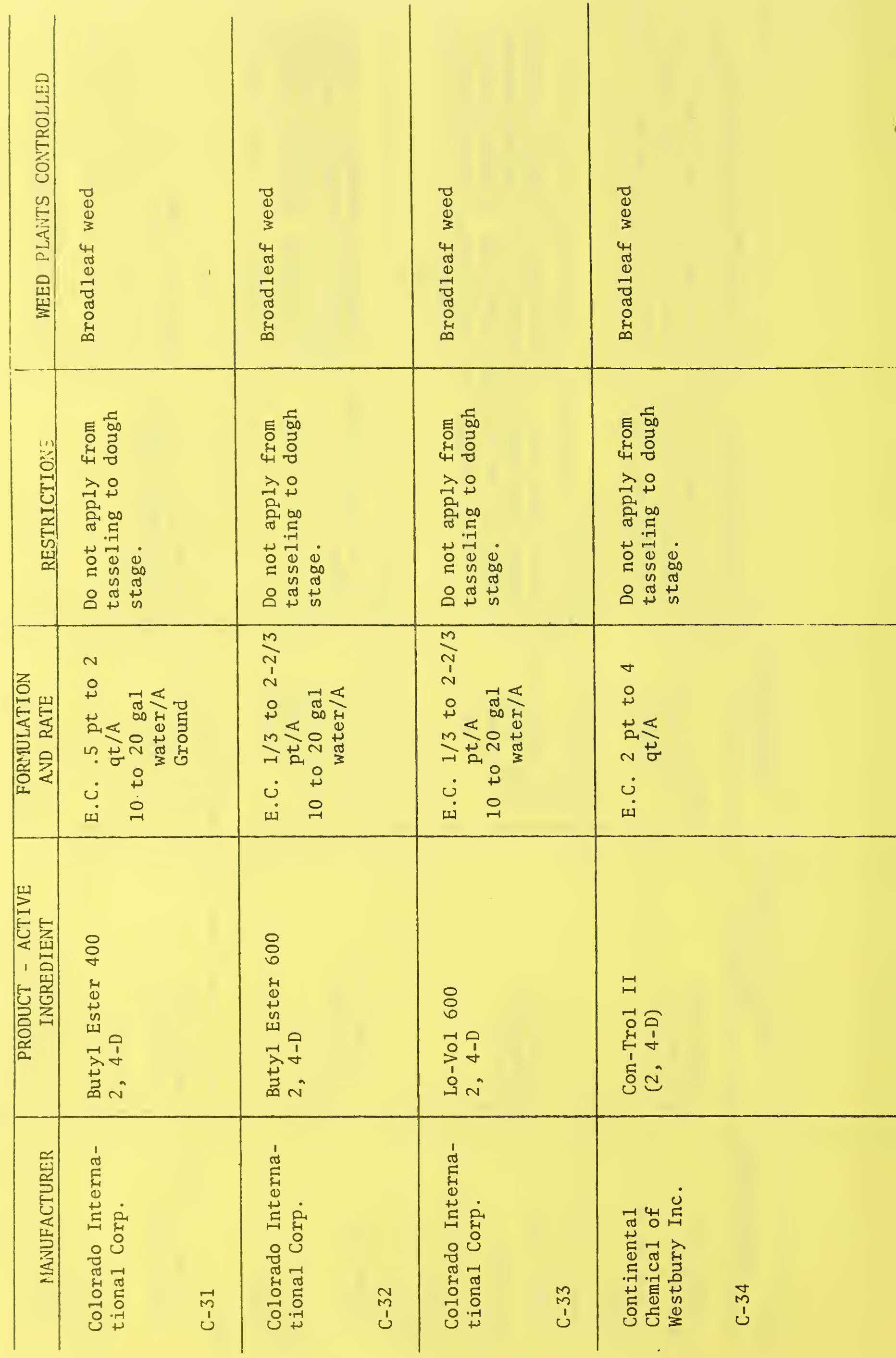




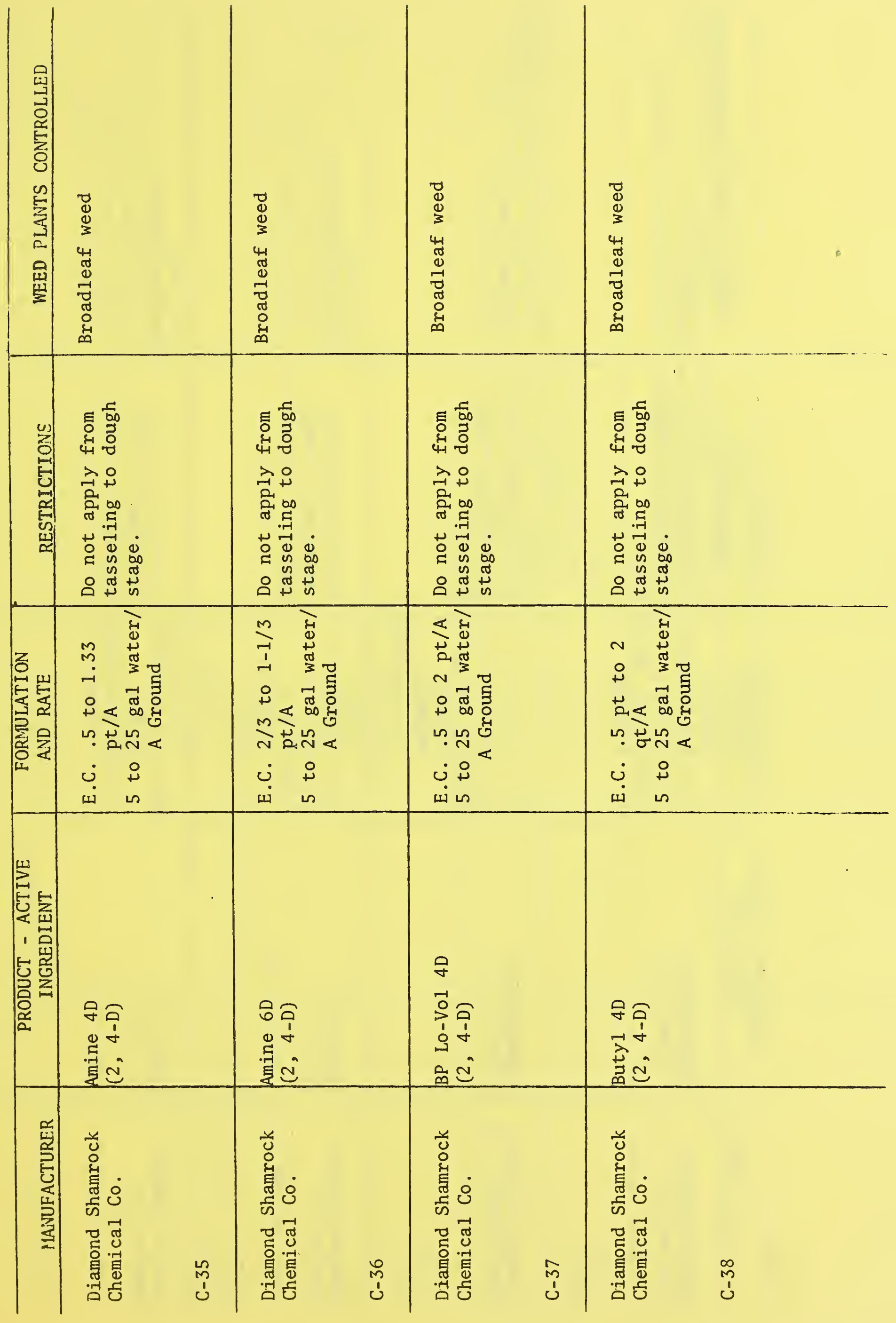




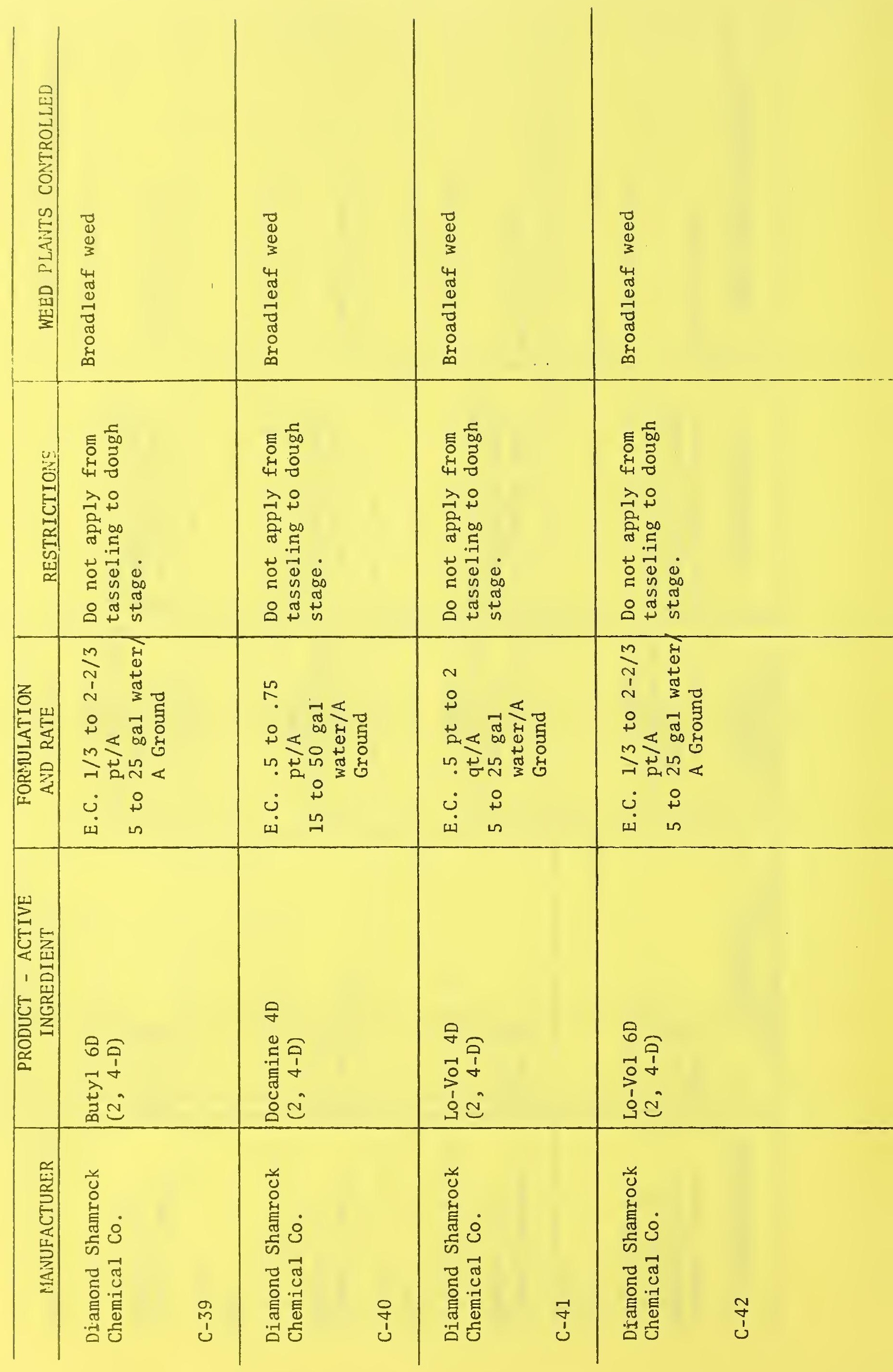




\begin{tabular}{|c|c|c|c|c|}
\hline 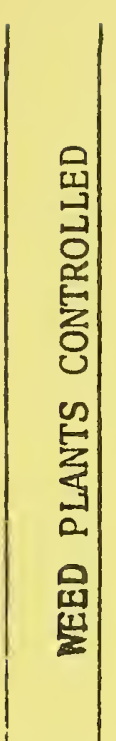 & 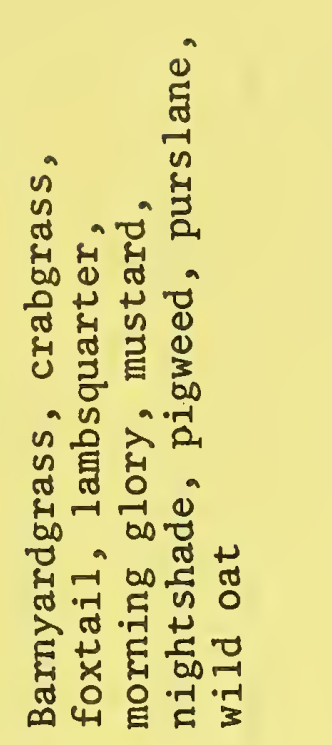 & 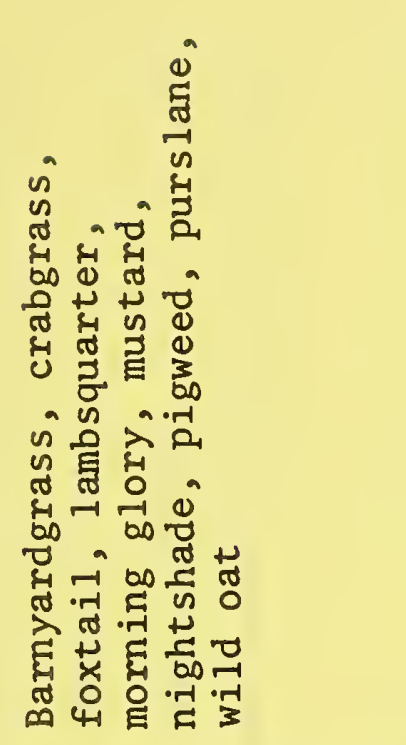 & 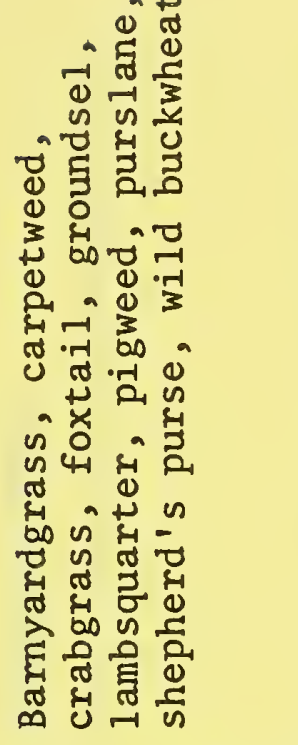 & 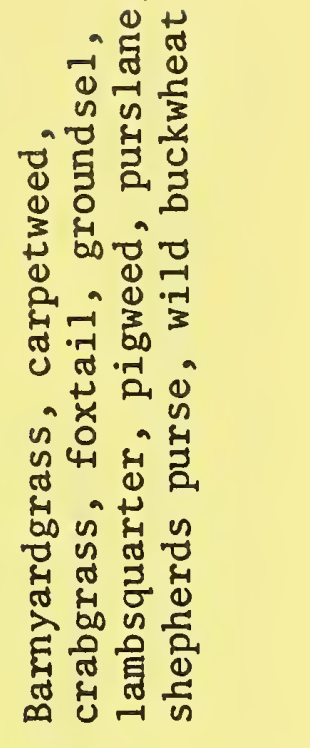 \\
\hline 을 & 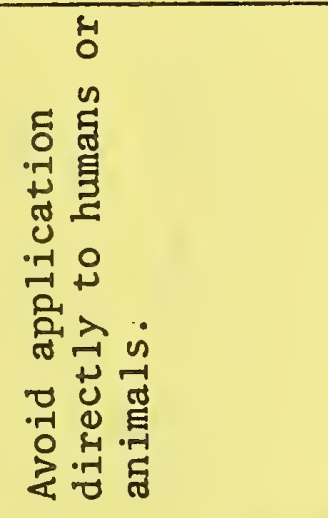 & 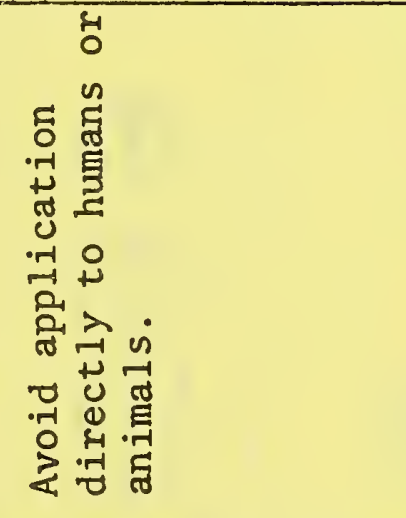 & 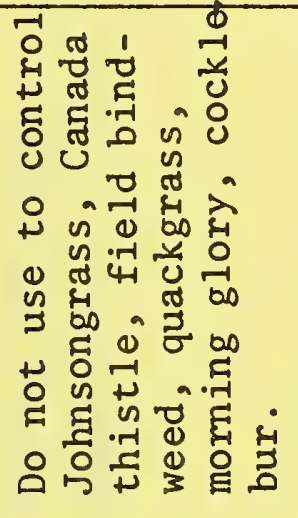 & 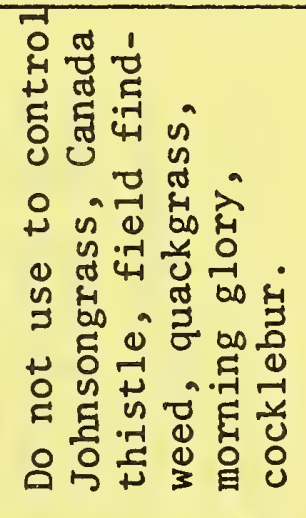 \\
\hline 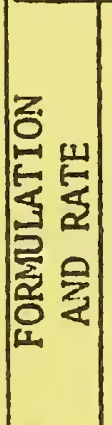 & 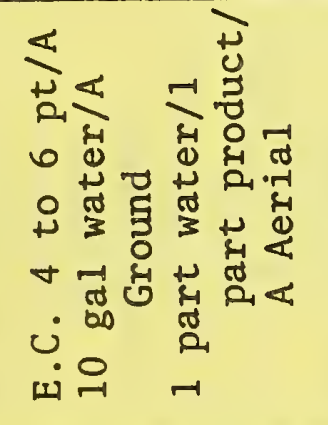 & $\begin{array}{l}m \\
\sim \\
\dot{n}\end{array}$ & 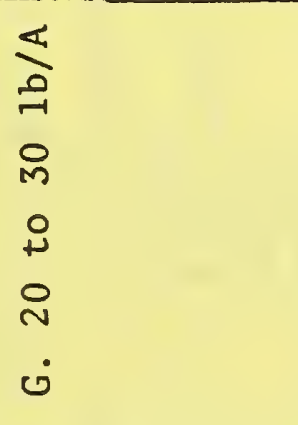 & 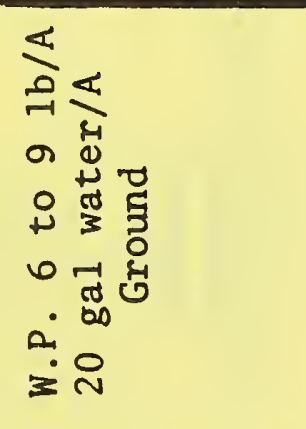 \\
\hline 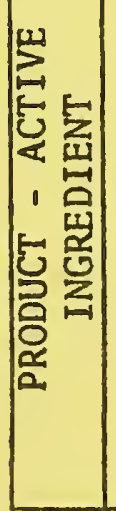 & 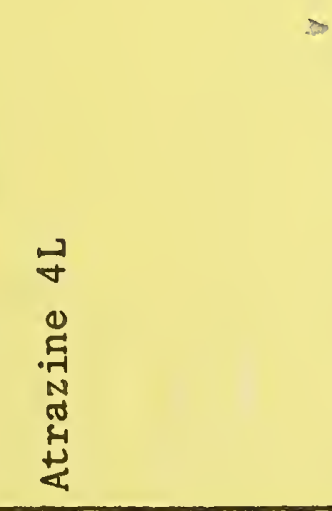 & 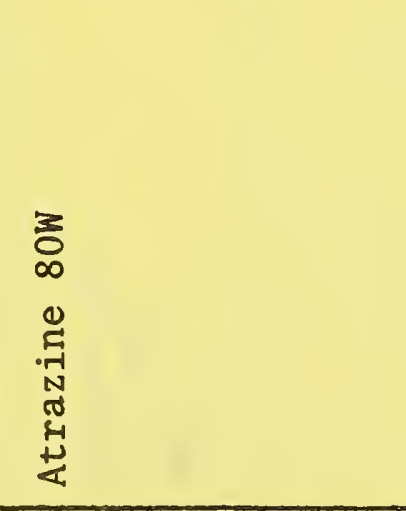 & 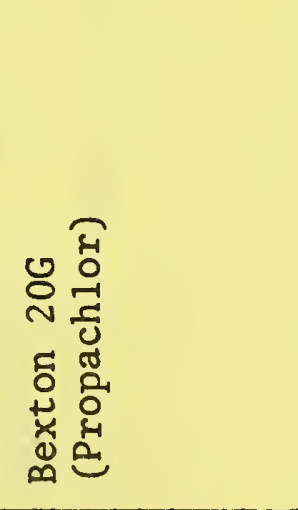 & 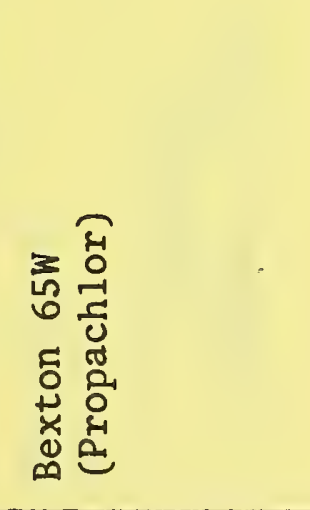 \\
\hline 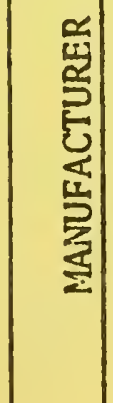 & 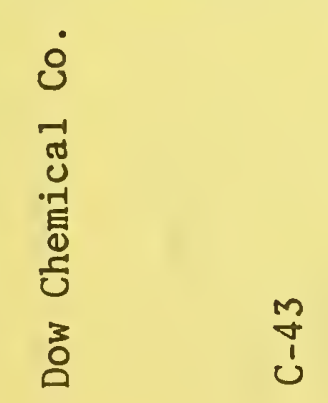 & 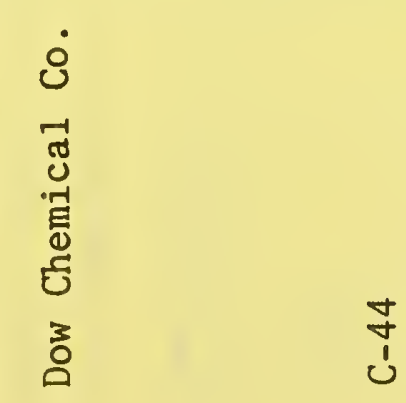 & 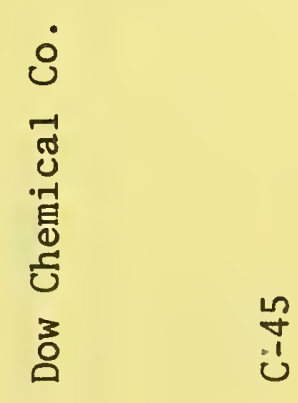 & 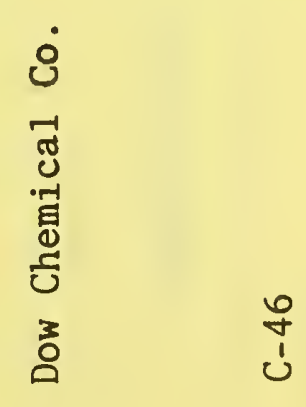 \\
\hline
\end{tabular}




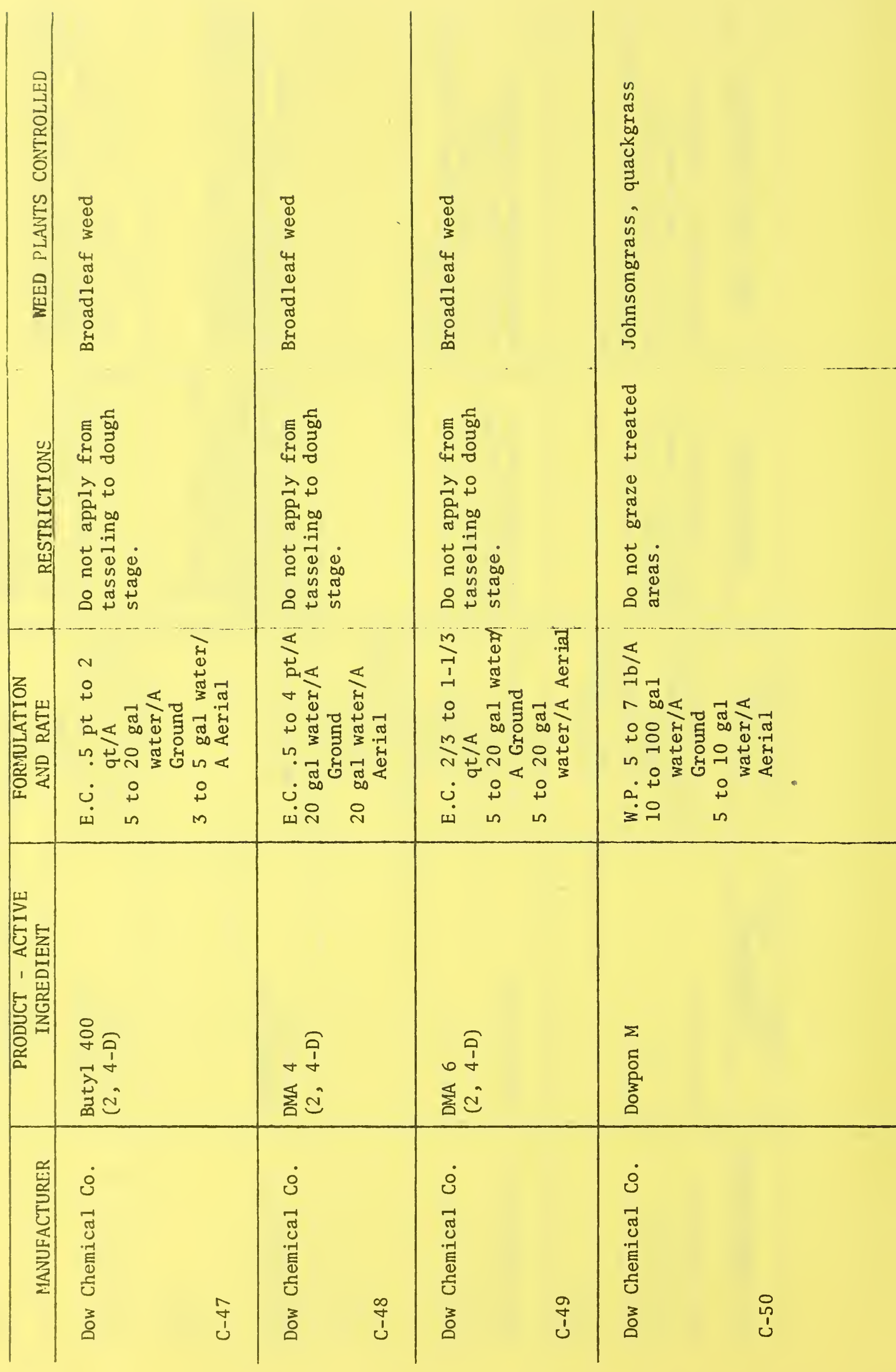




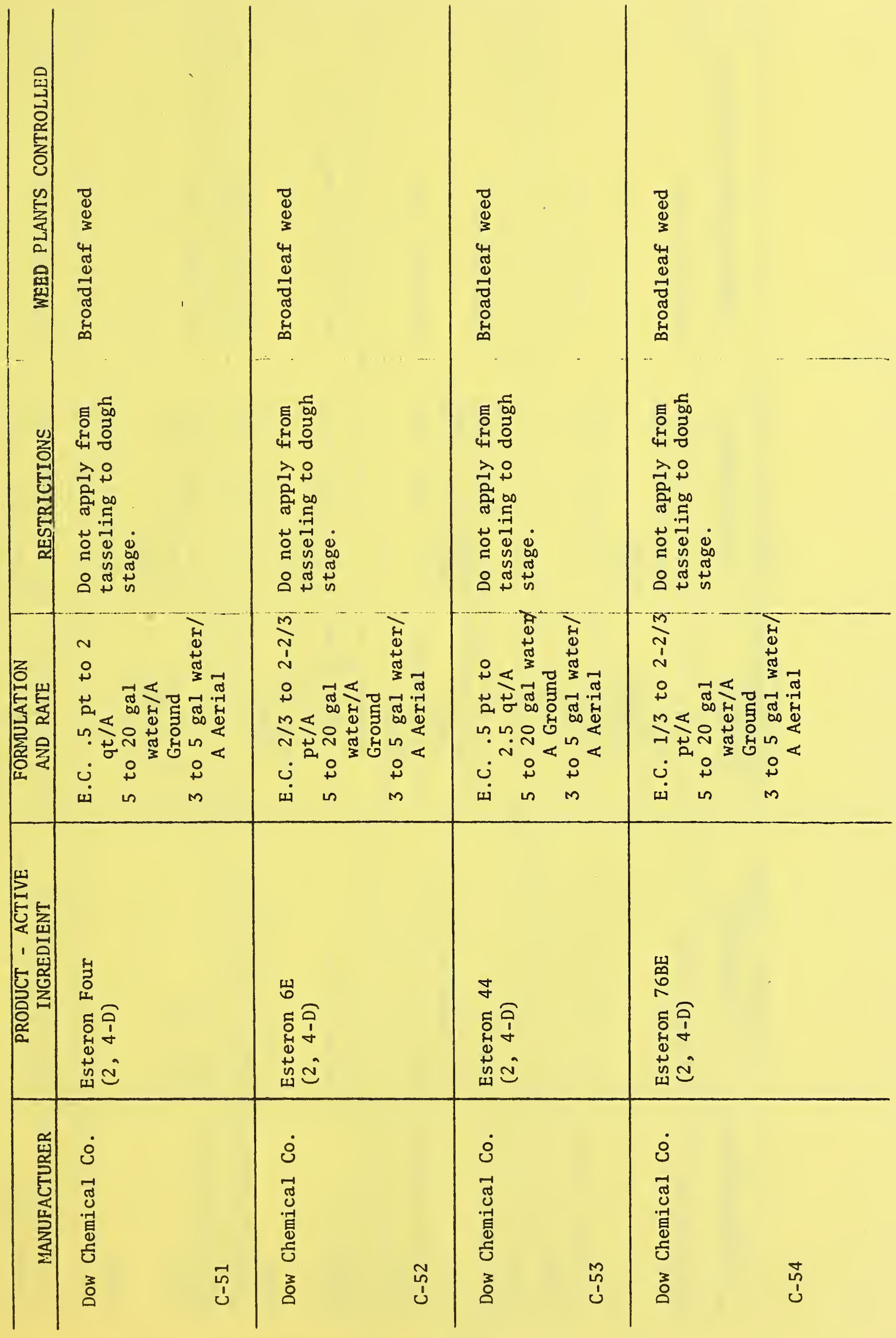




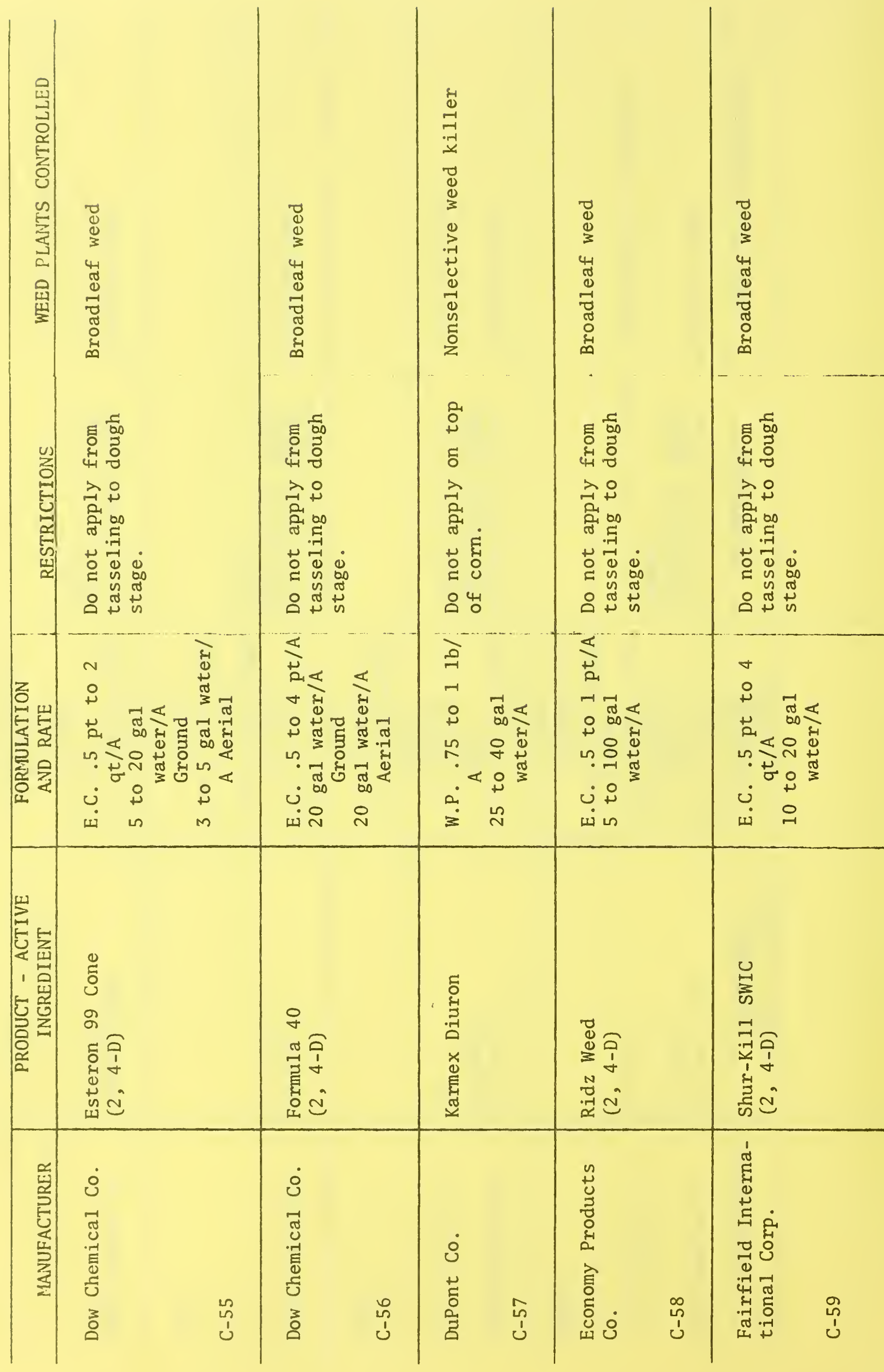




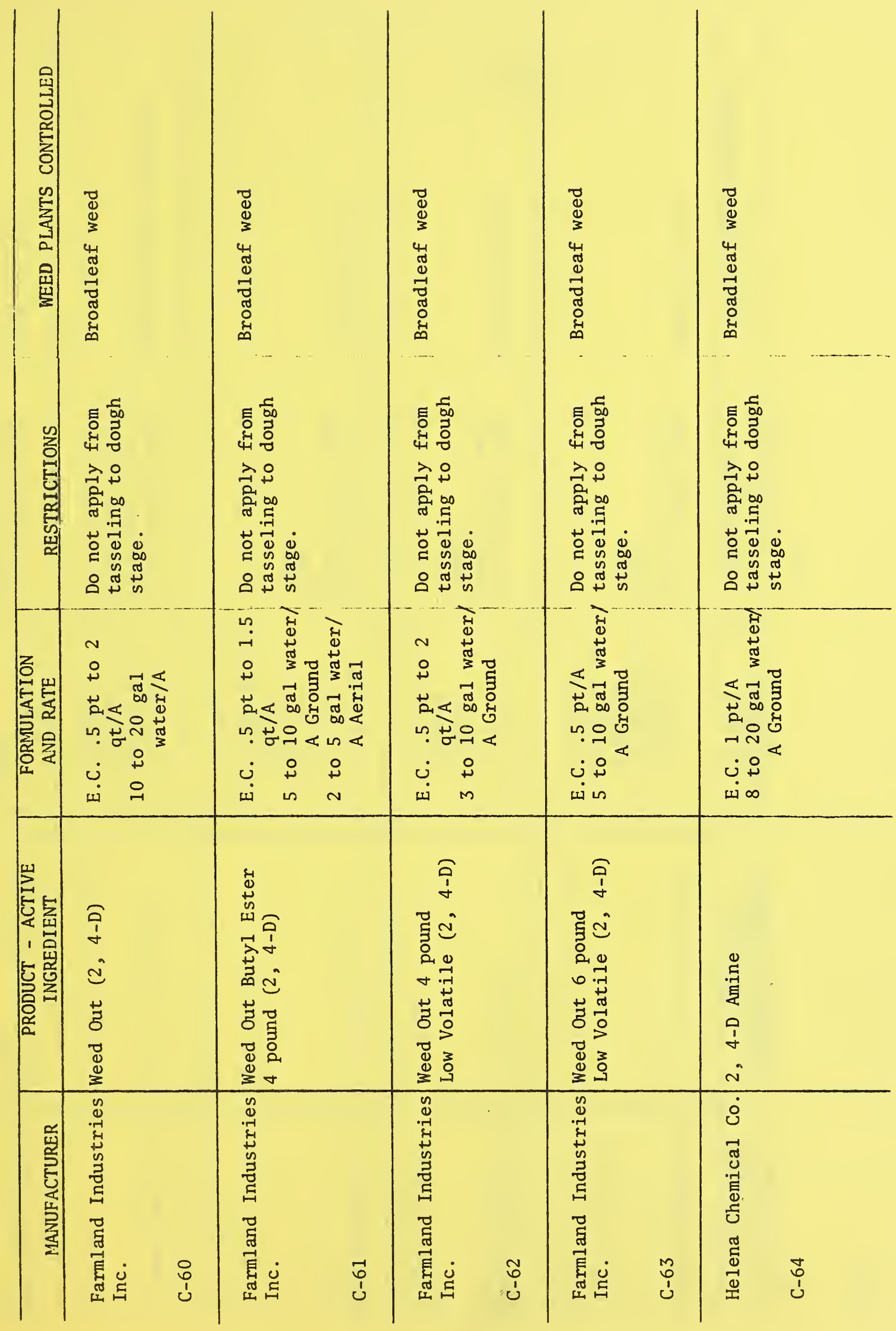




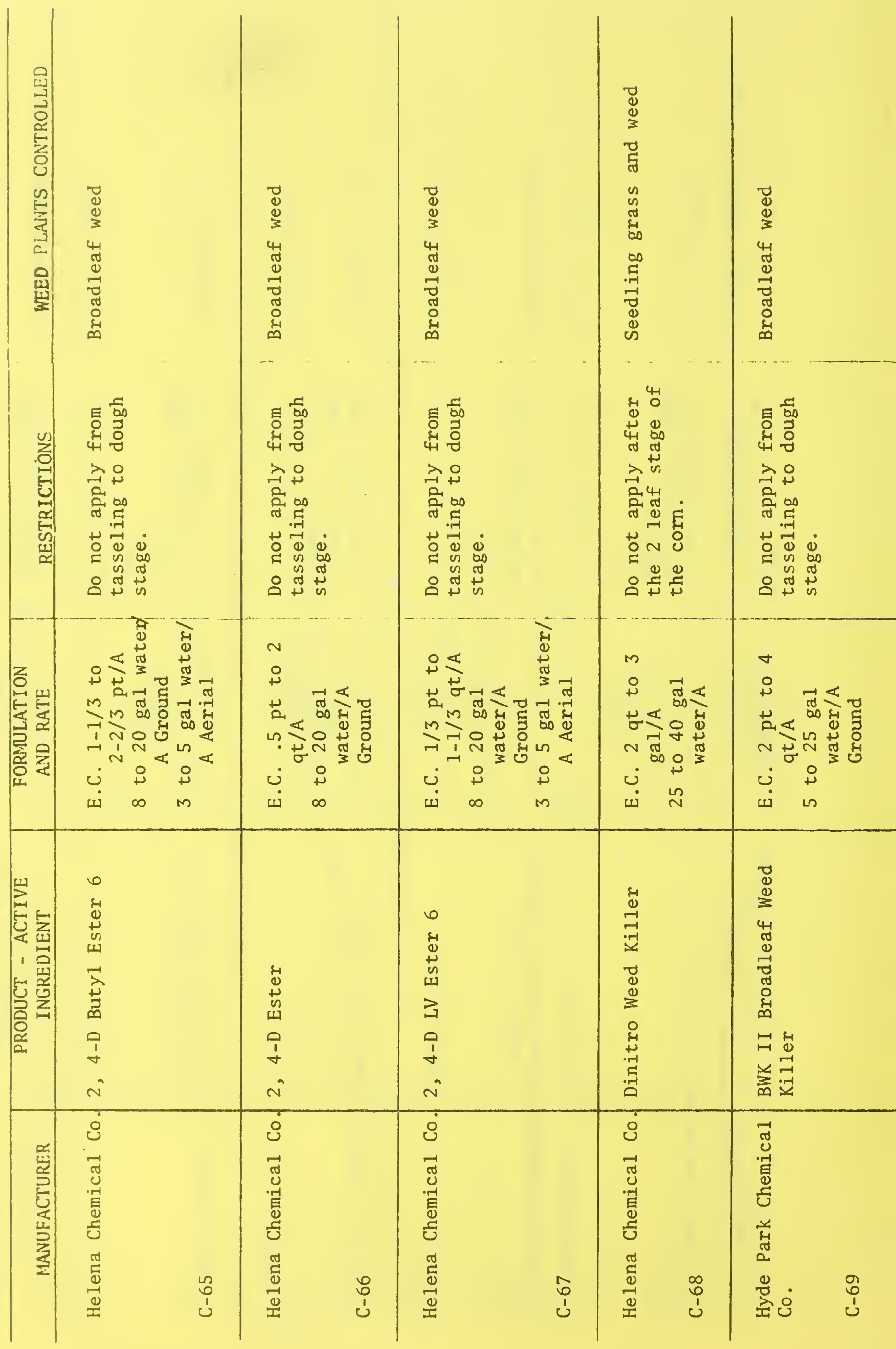




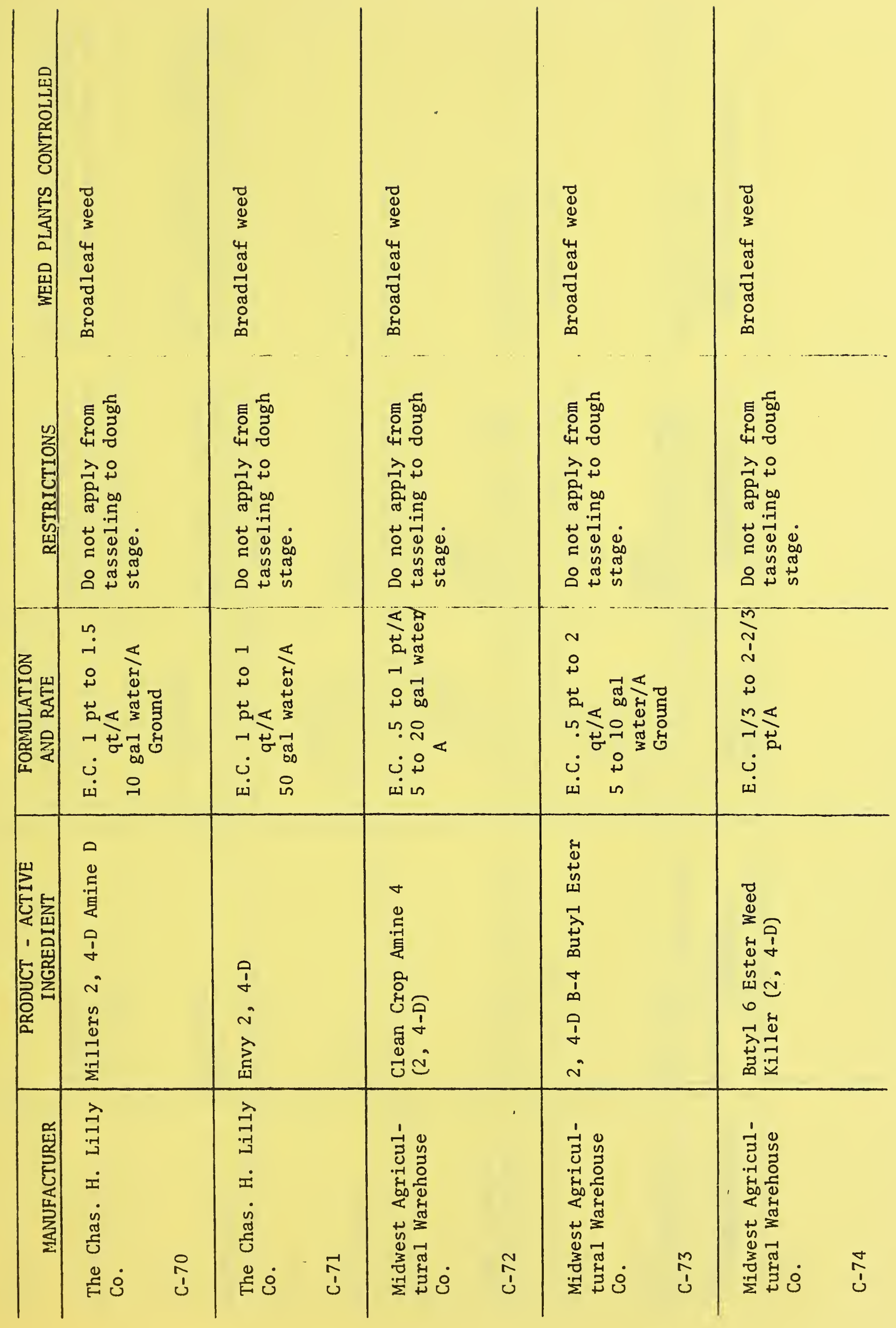




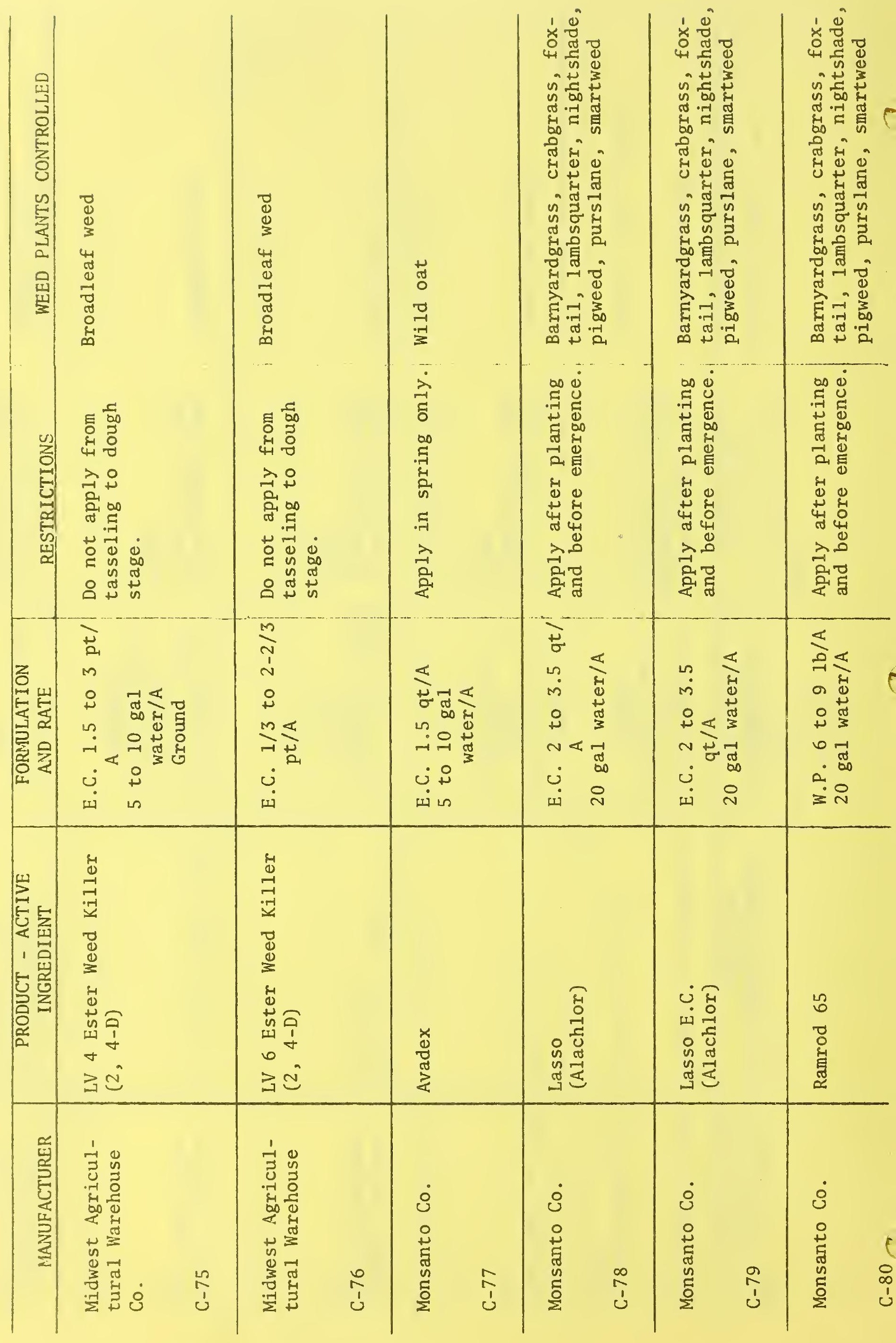




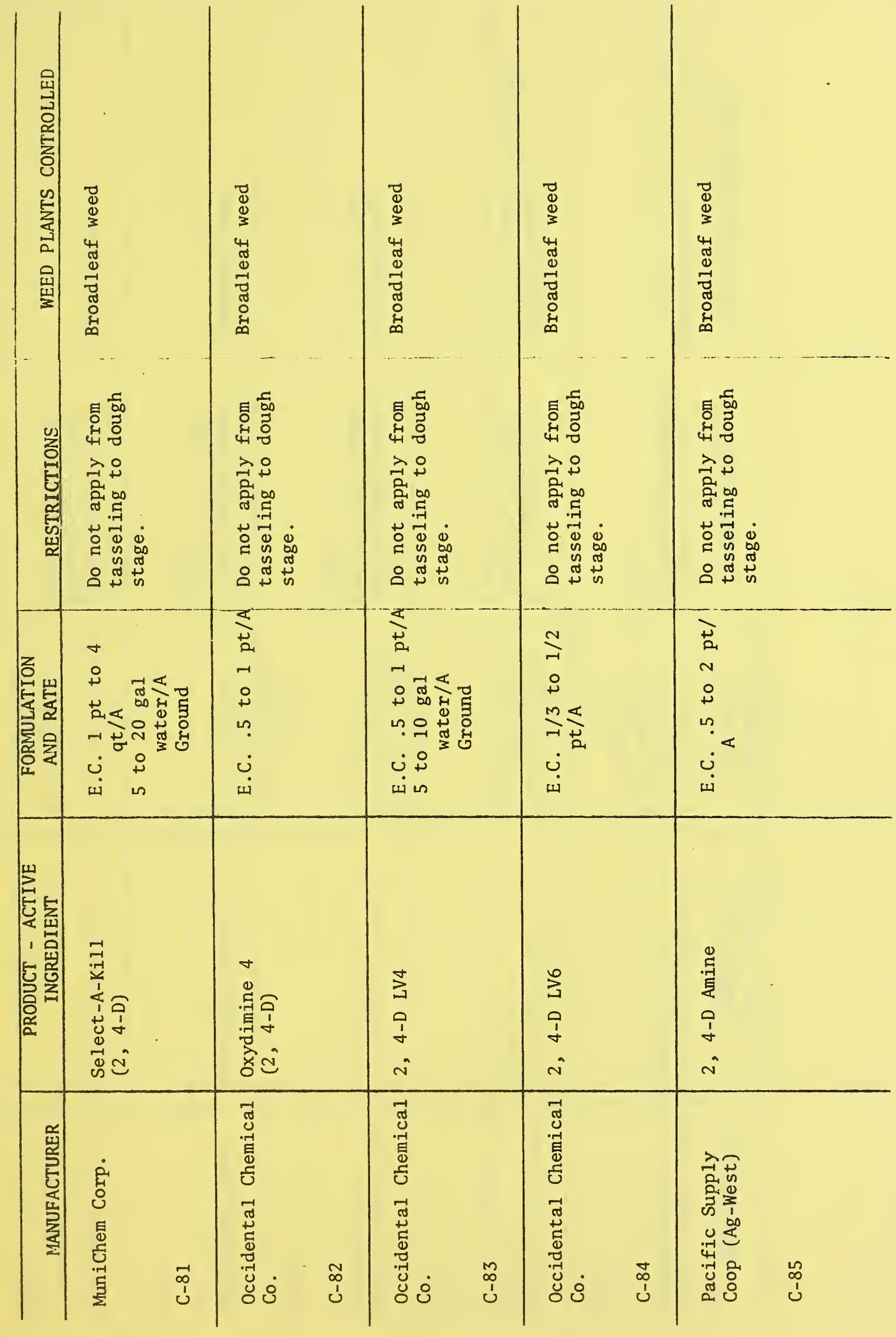




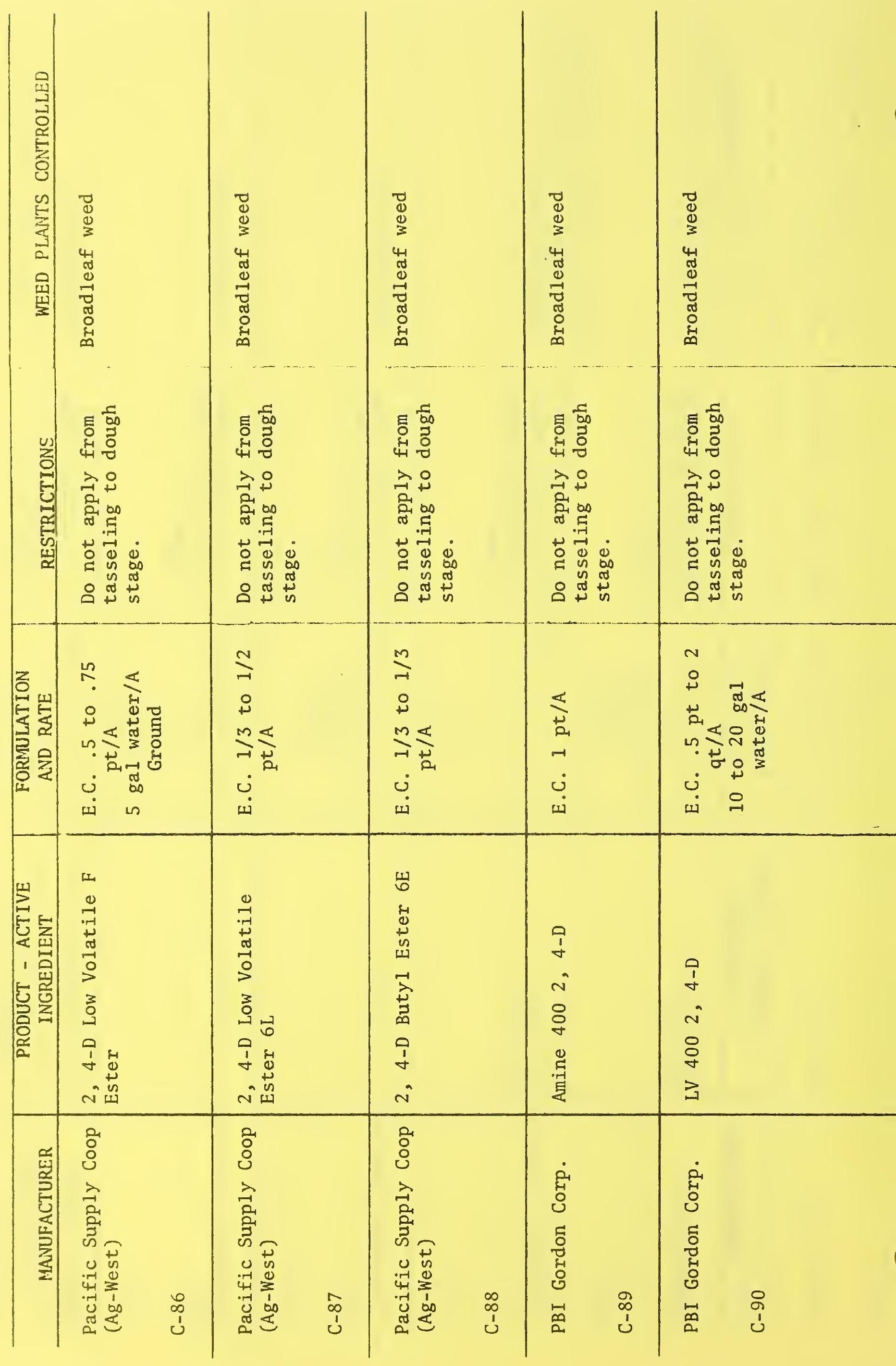




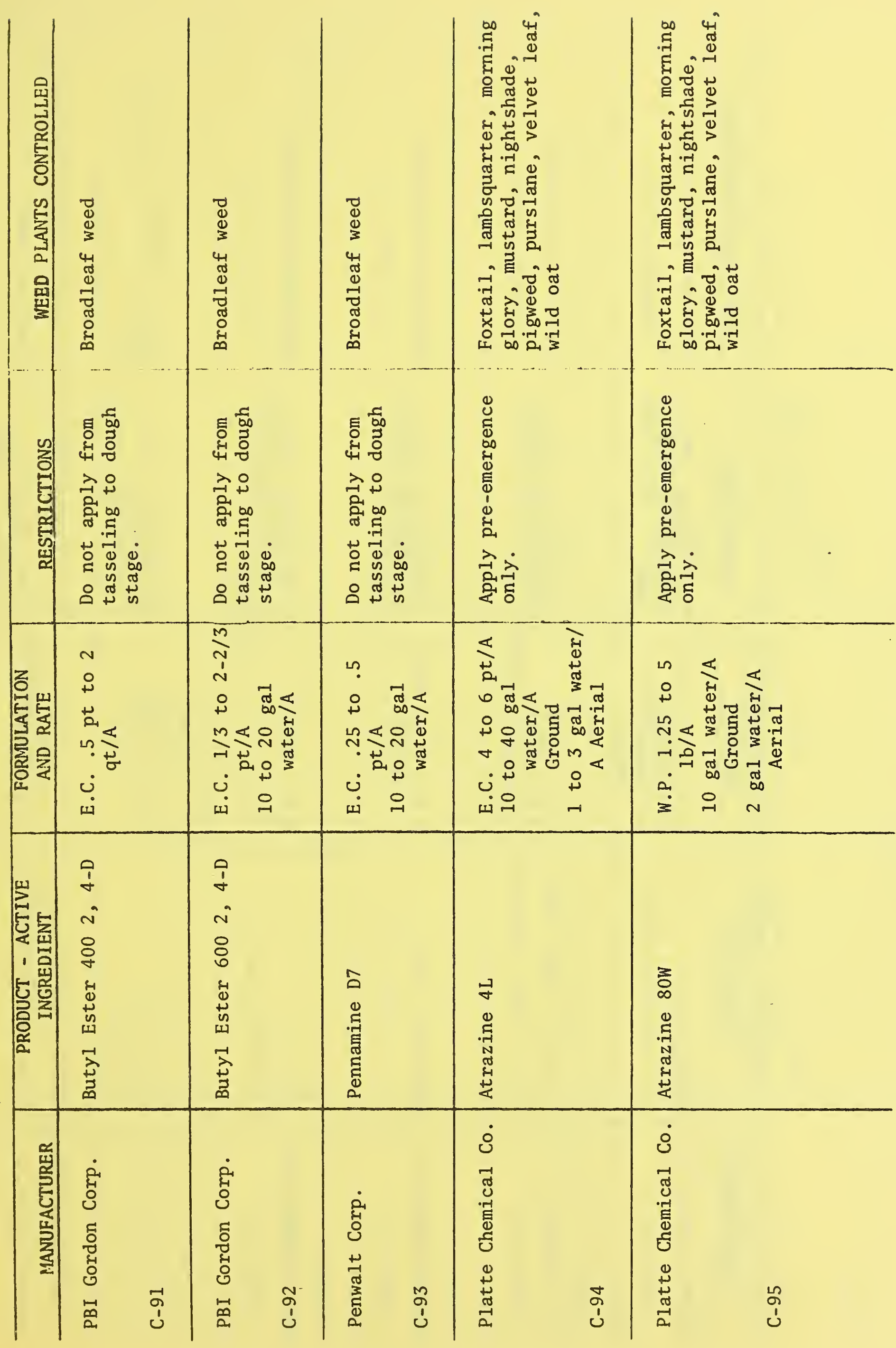




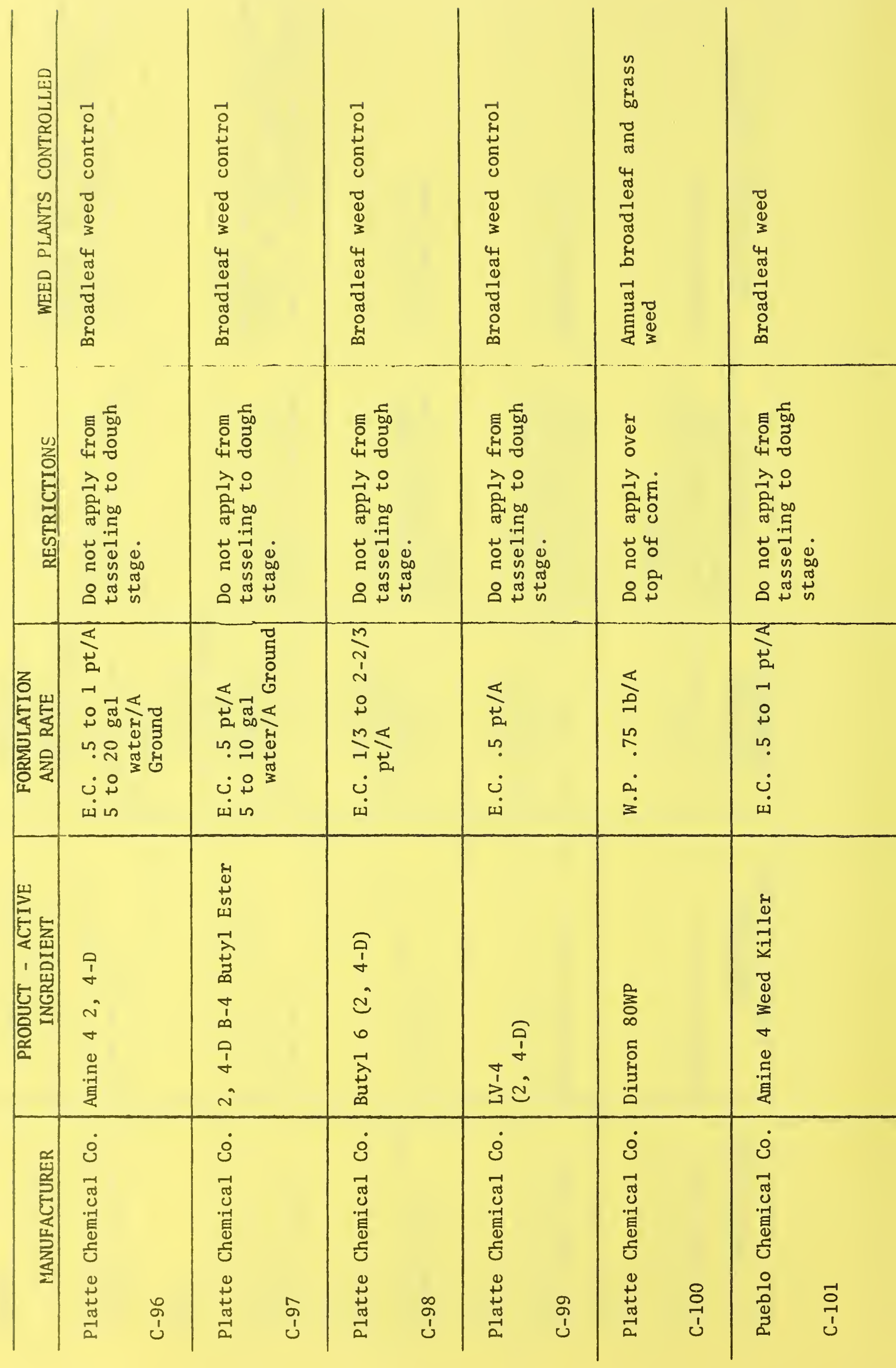




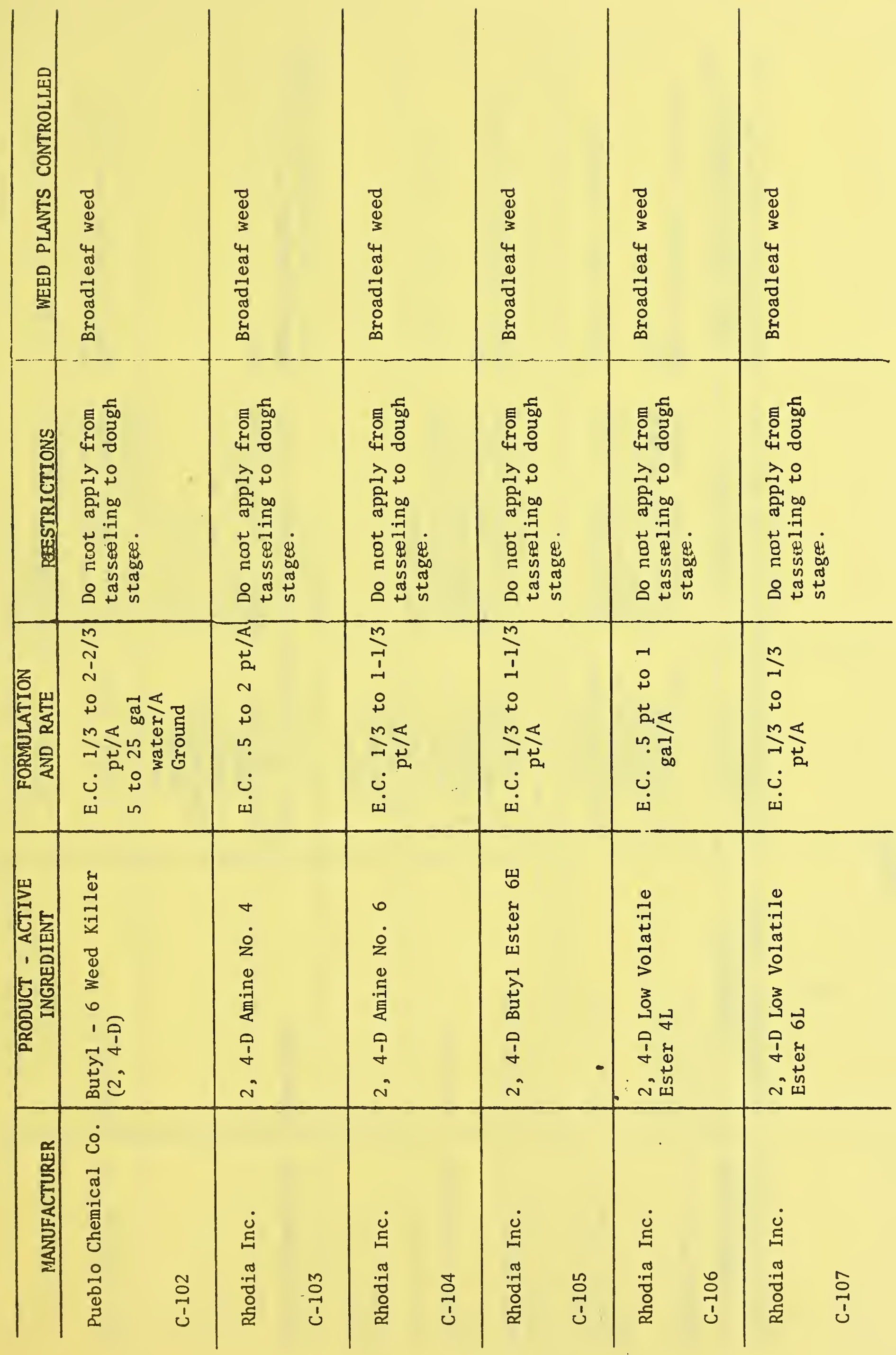




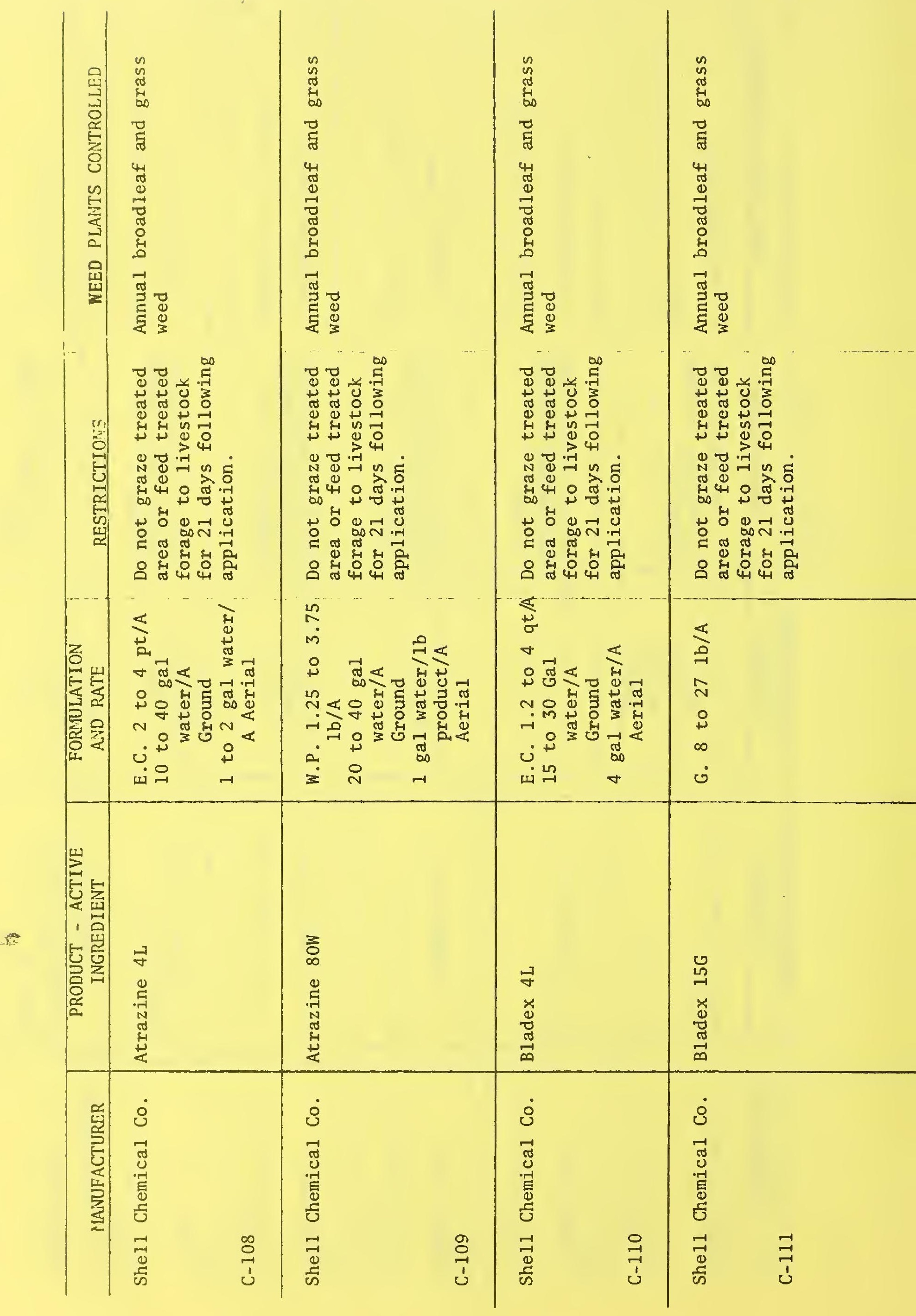




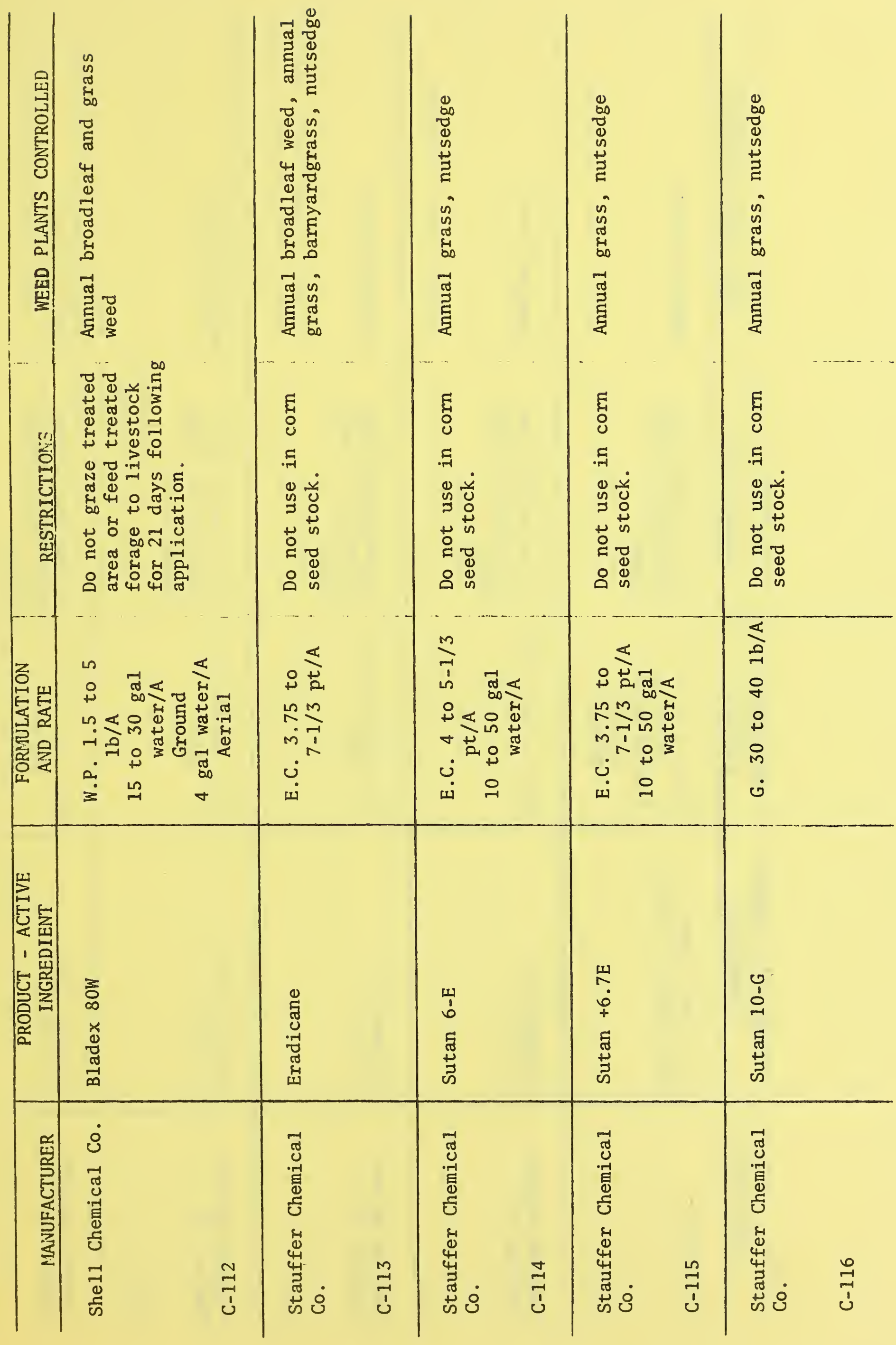




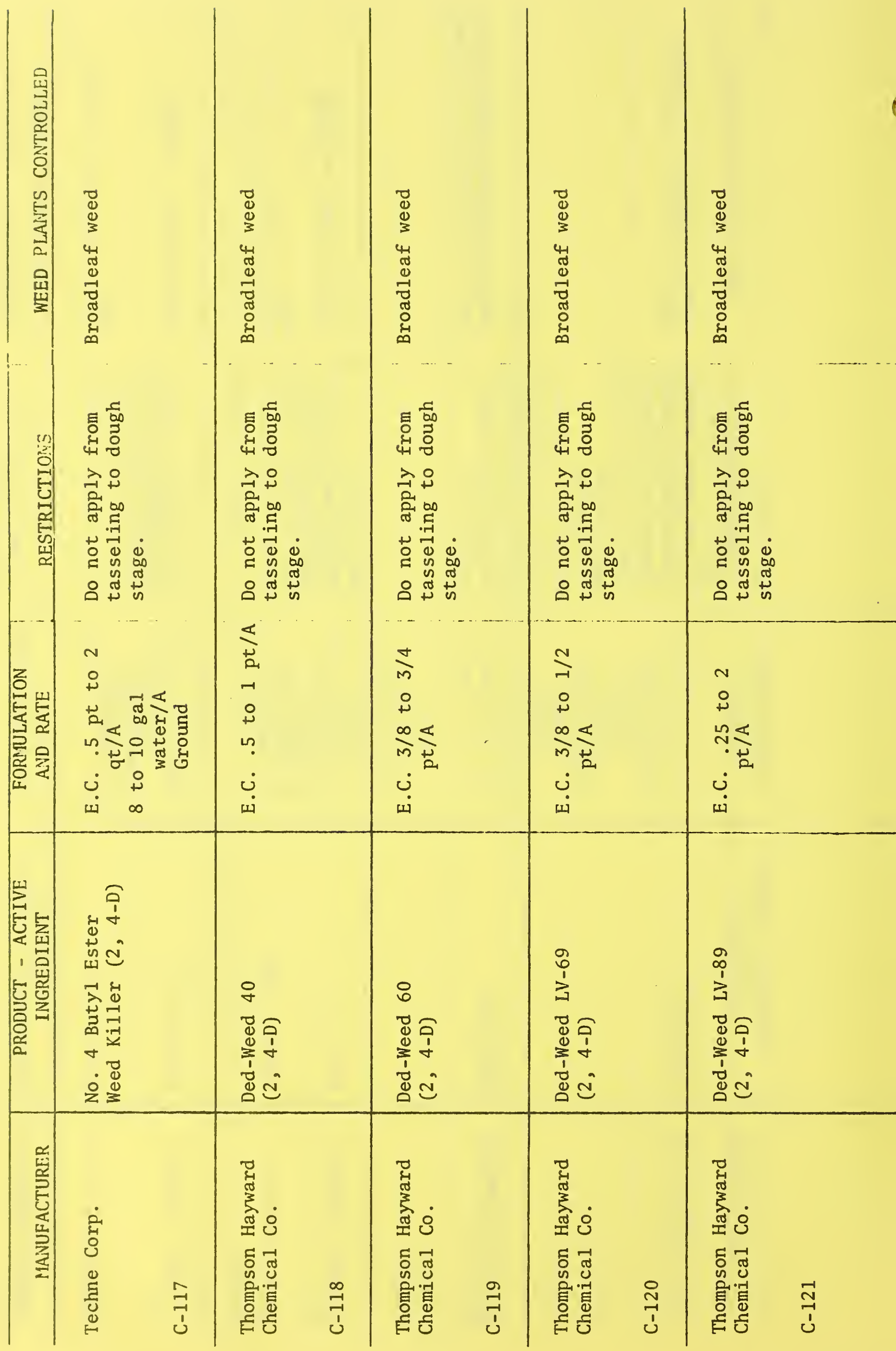




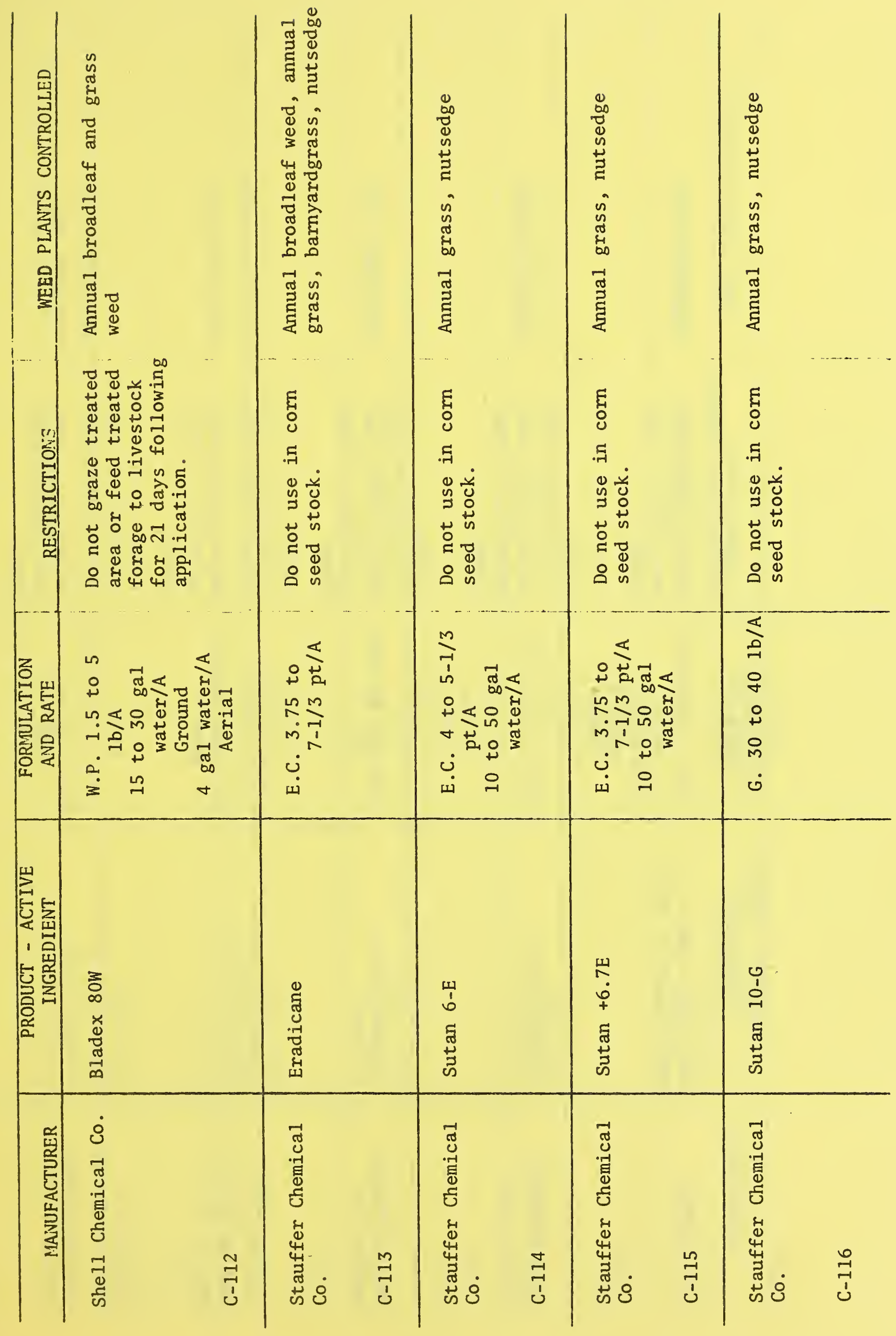




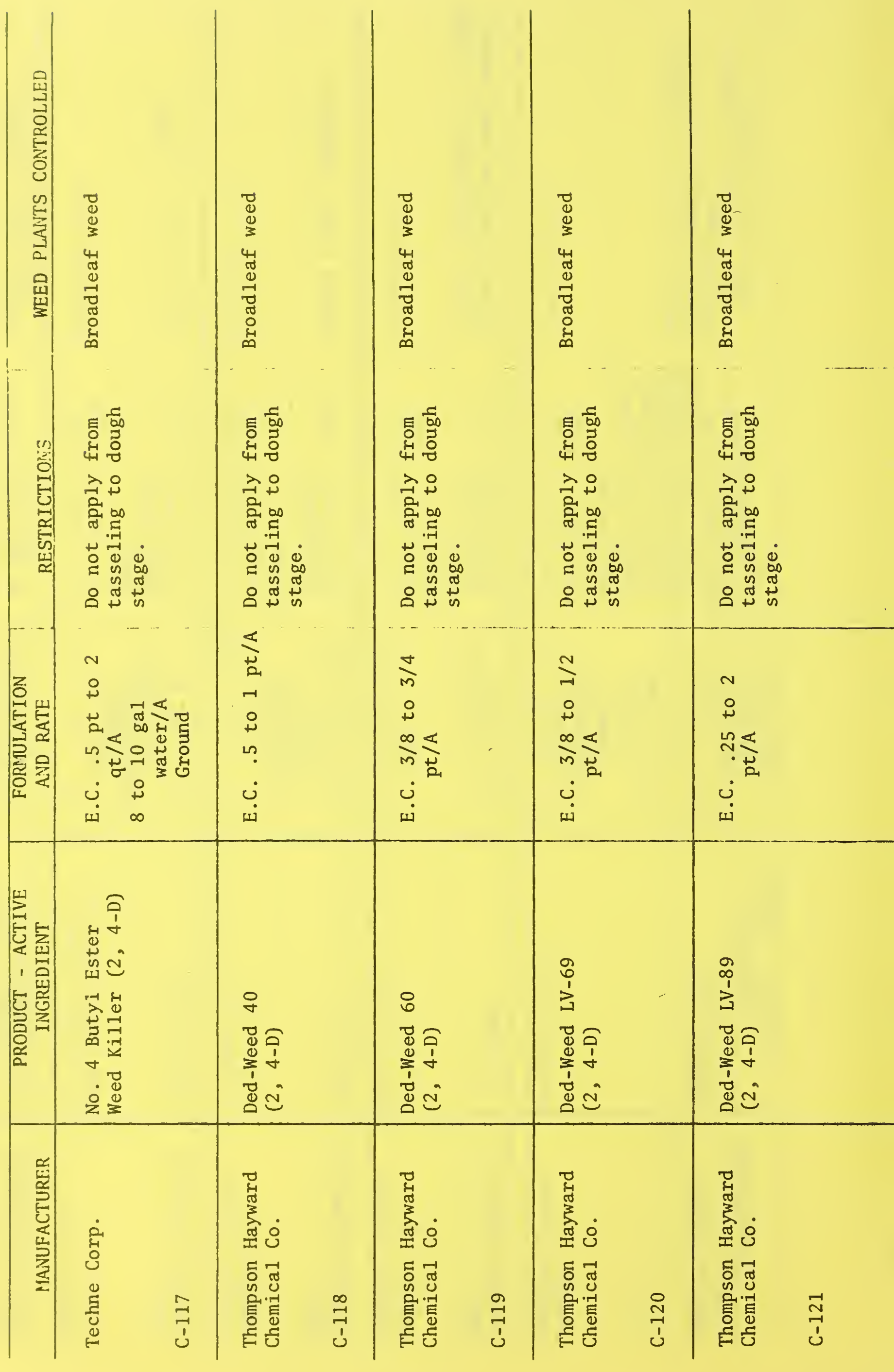




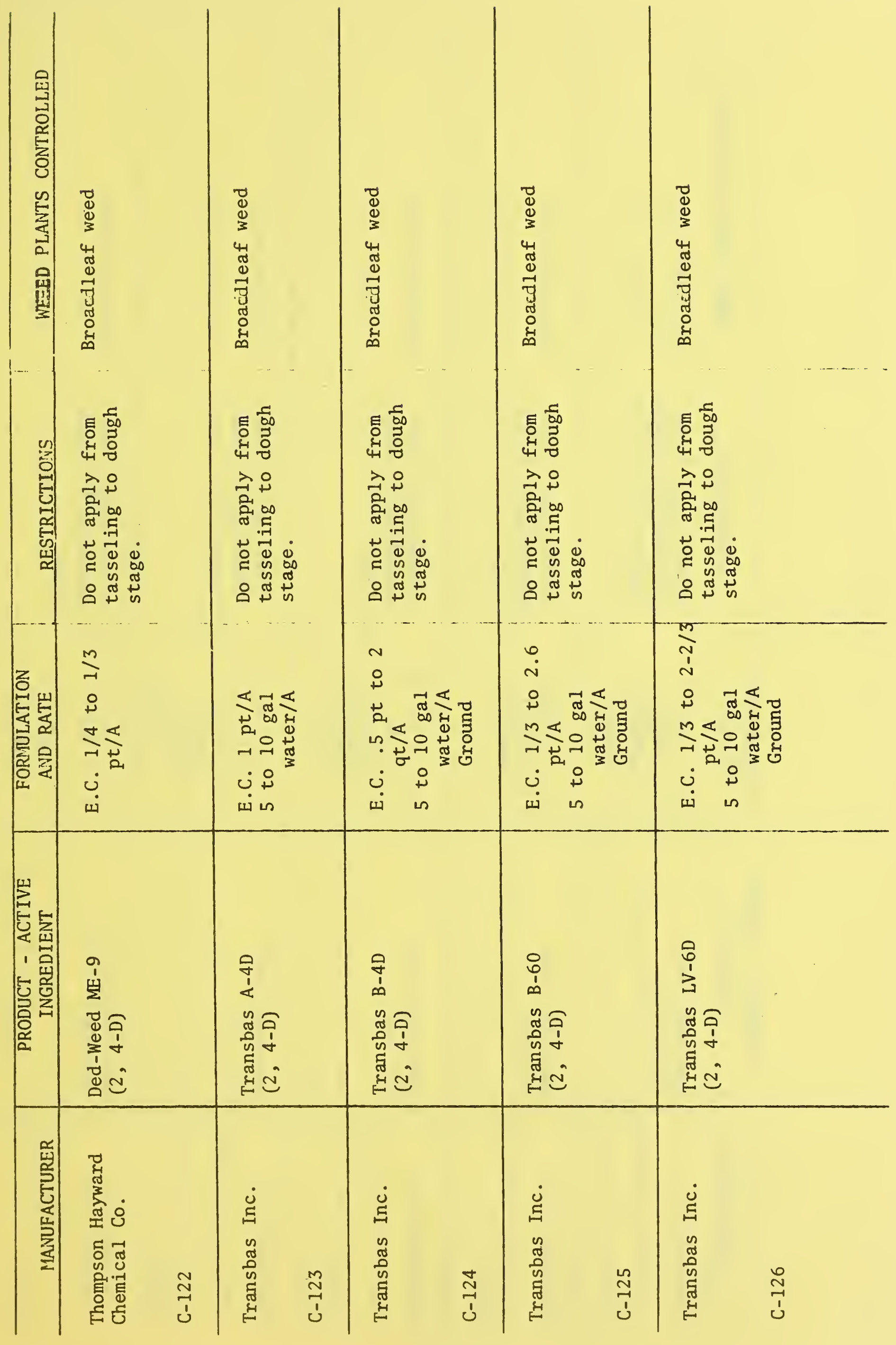




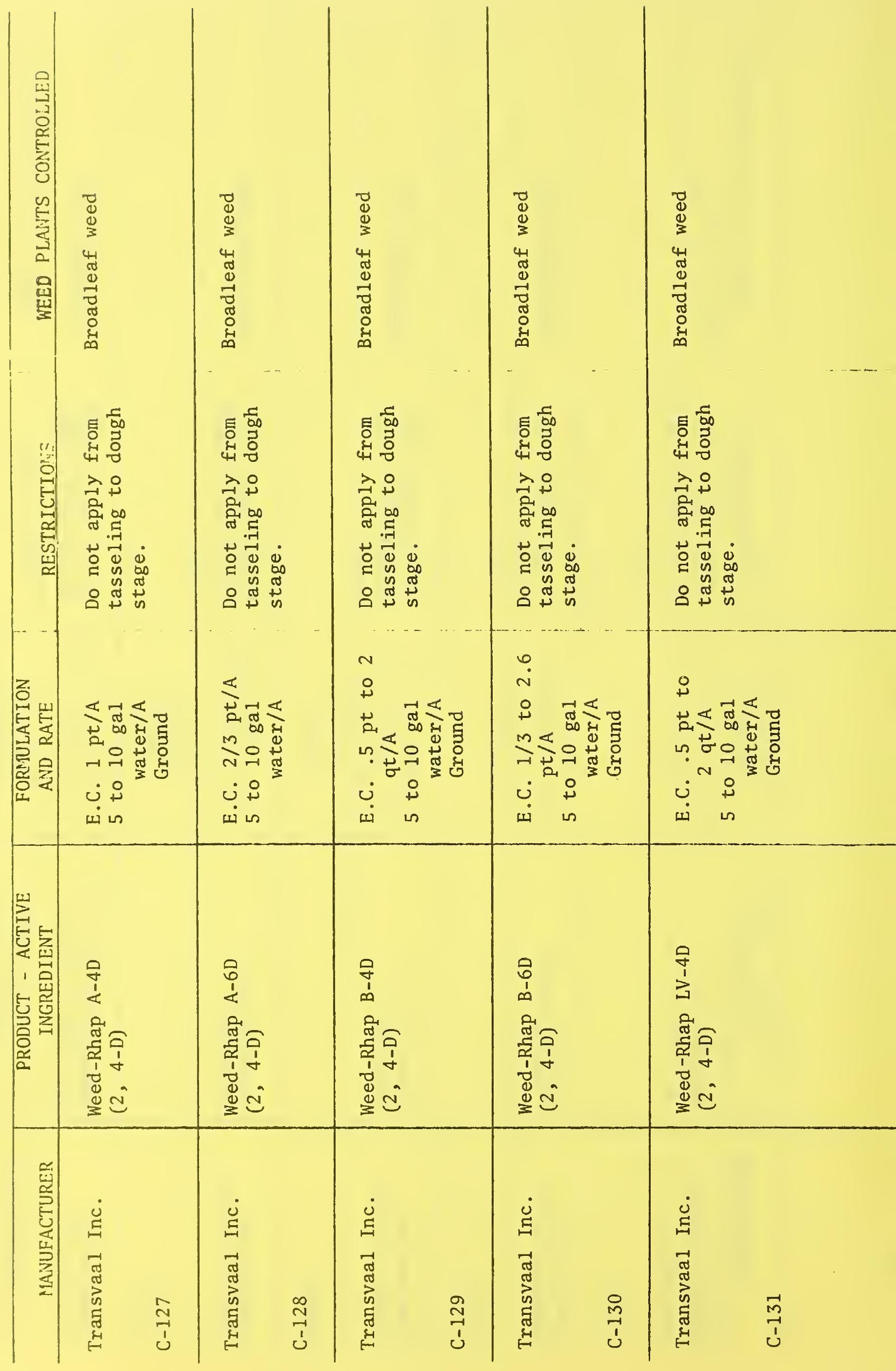




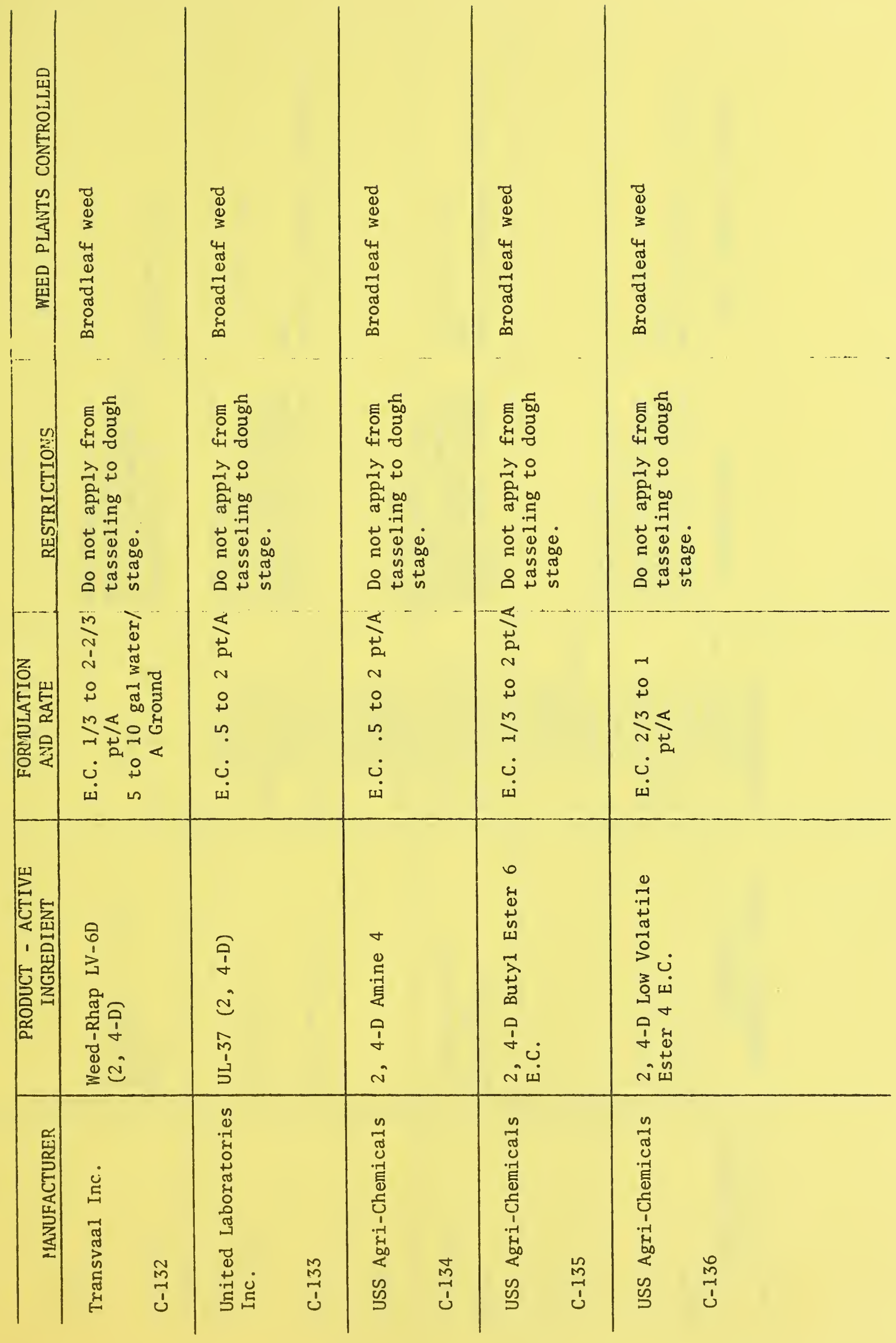




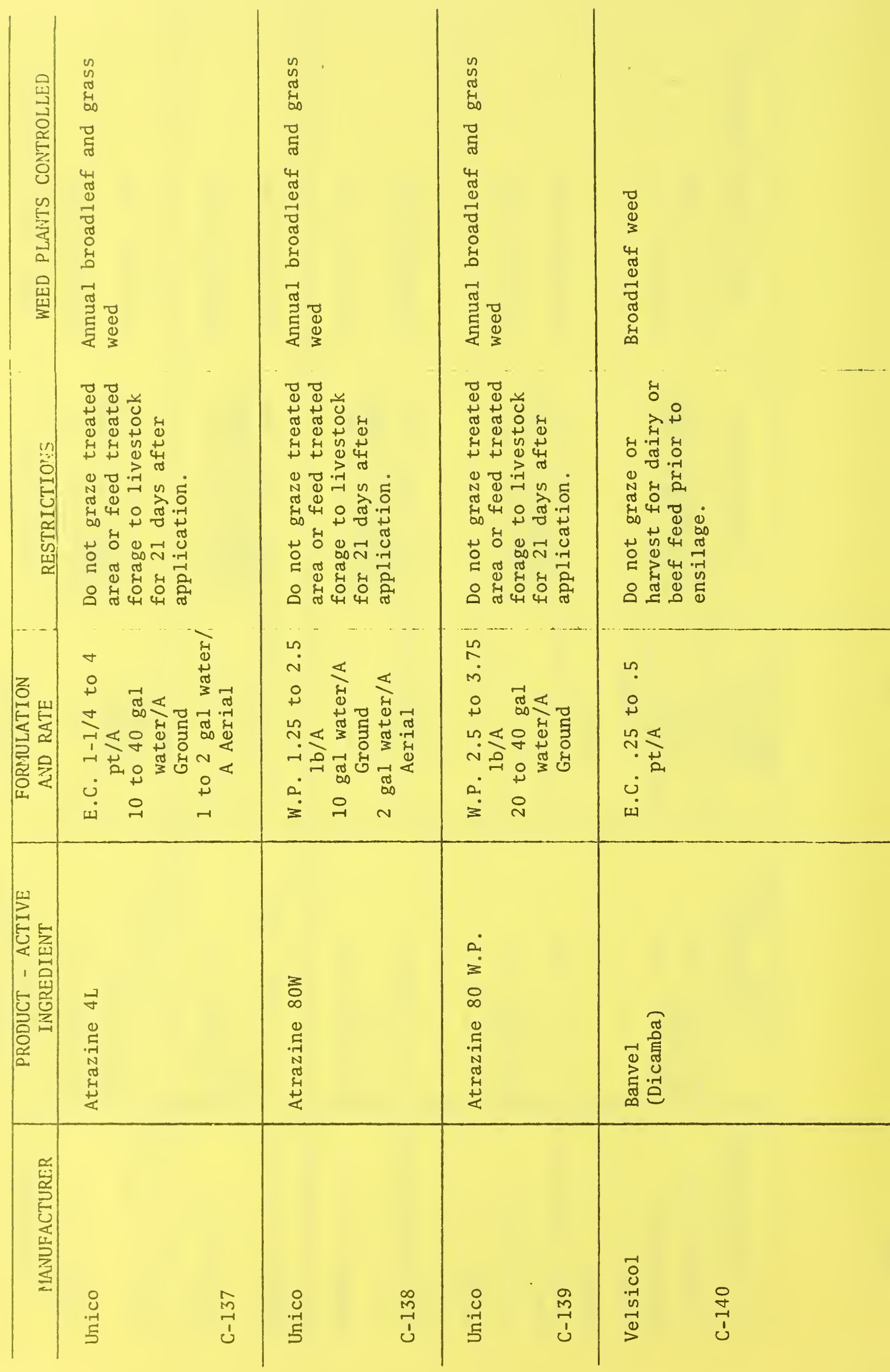




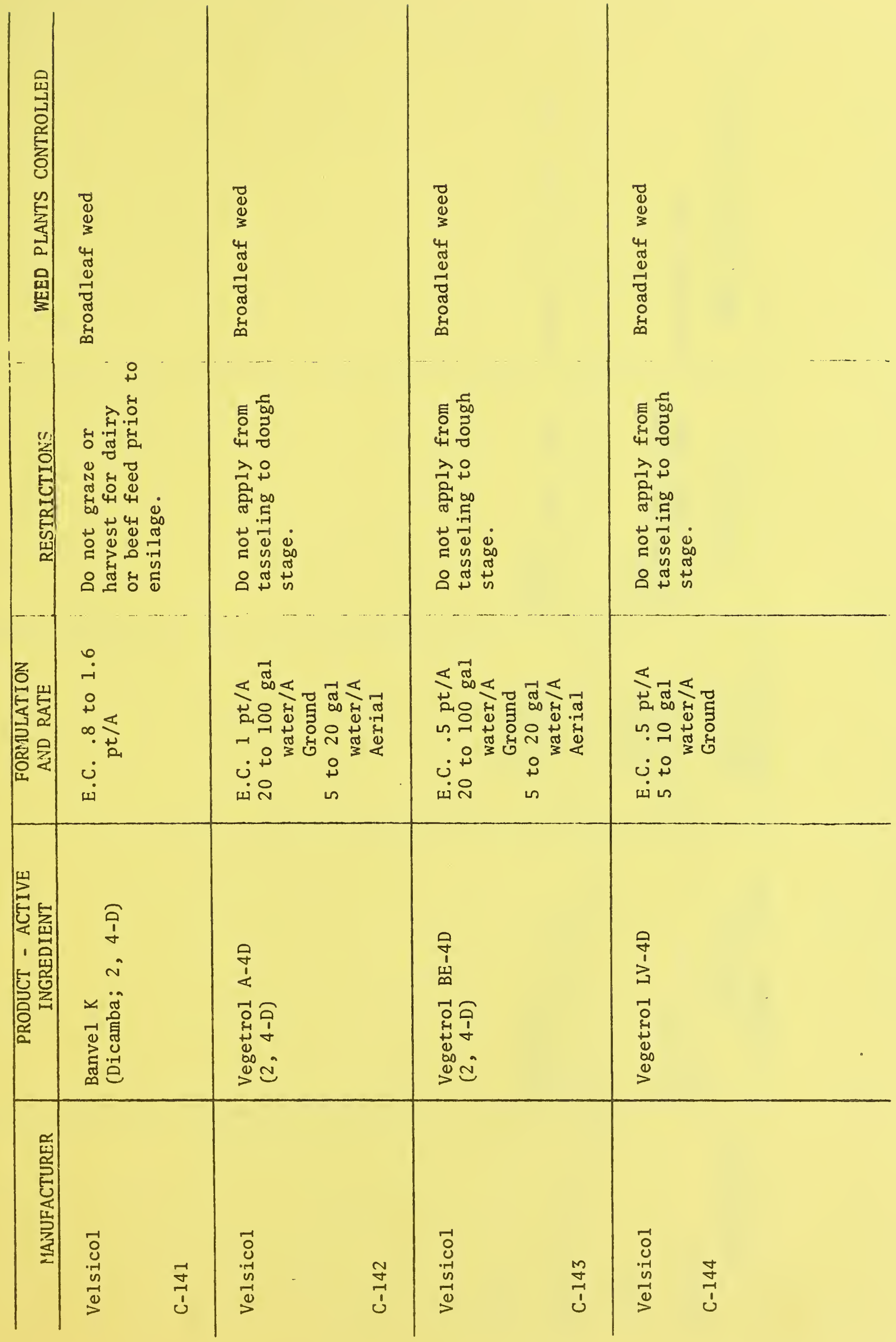




\begin{tabular}{|c|c|c|}
\hline 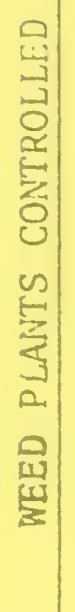 & 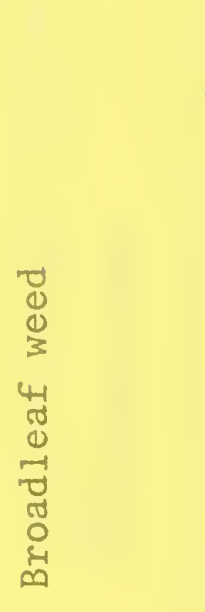 & 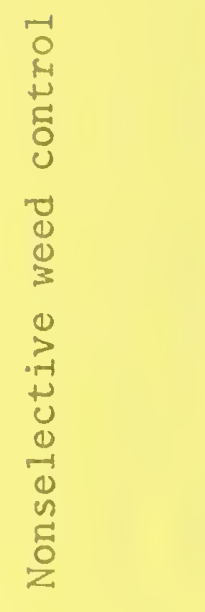 \\
\hline 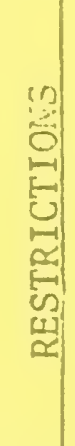 & 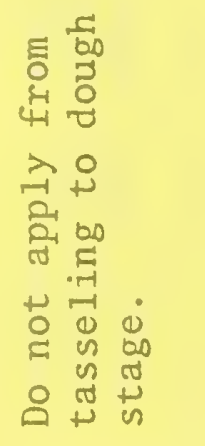 & 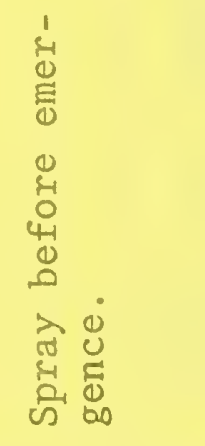 \\
\hline 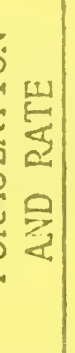 & 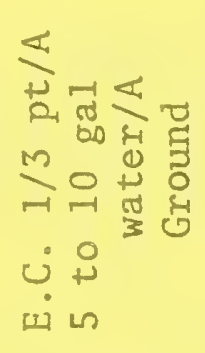 & 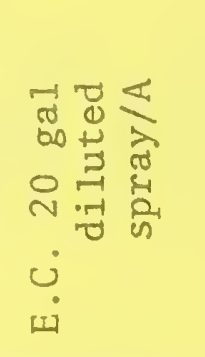 \\
\hline 氙 & 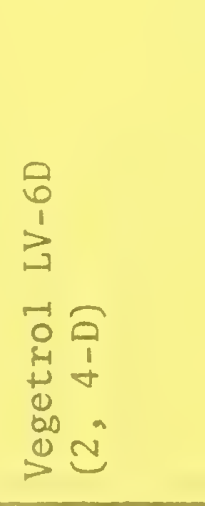 & 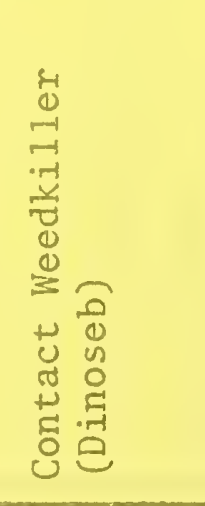 \\
\hline 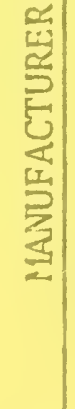 & 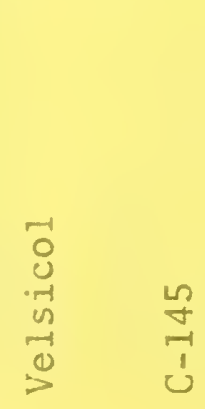 & 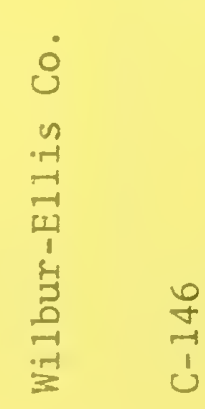 \\
\hline
\end{tabular}





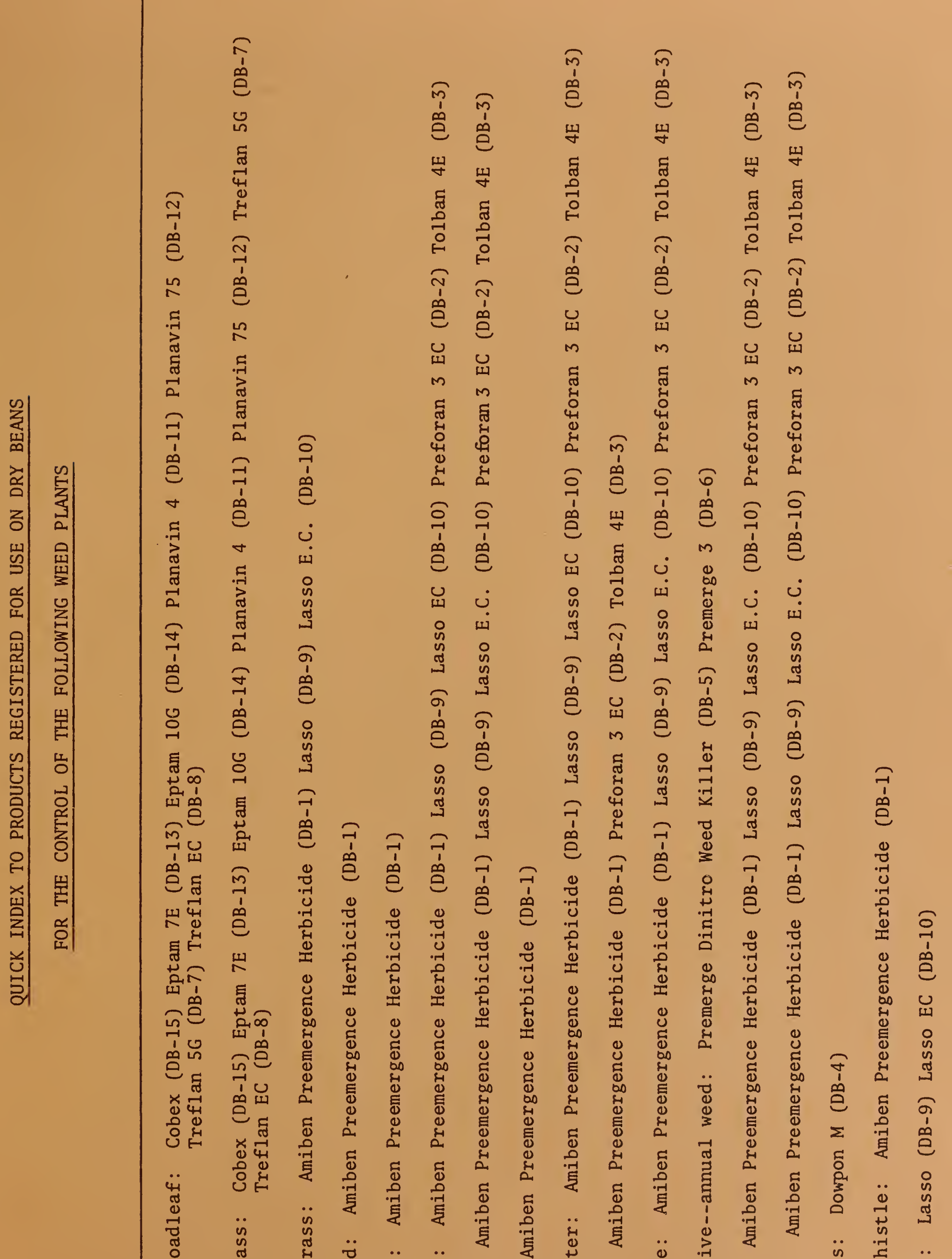

䱛

ए

总令

的急总金

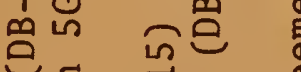

政

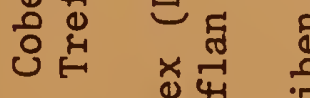

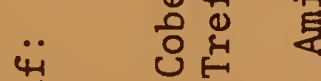

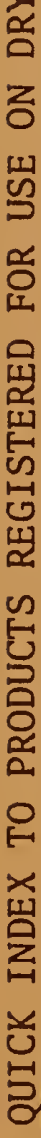

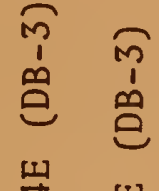

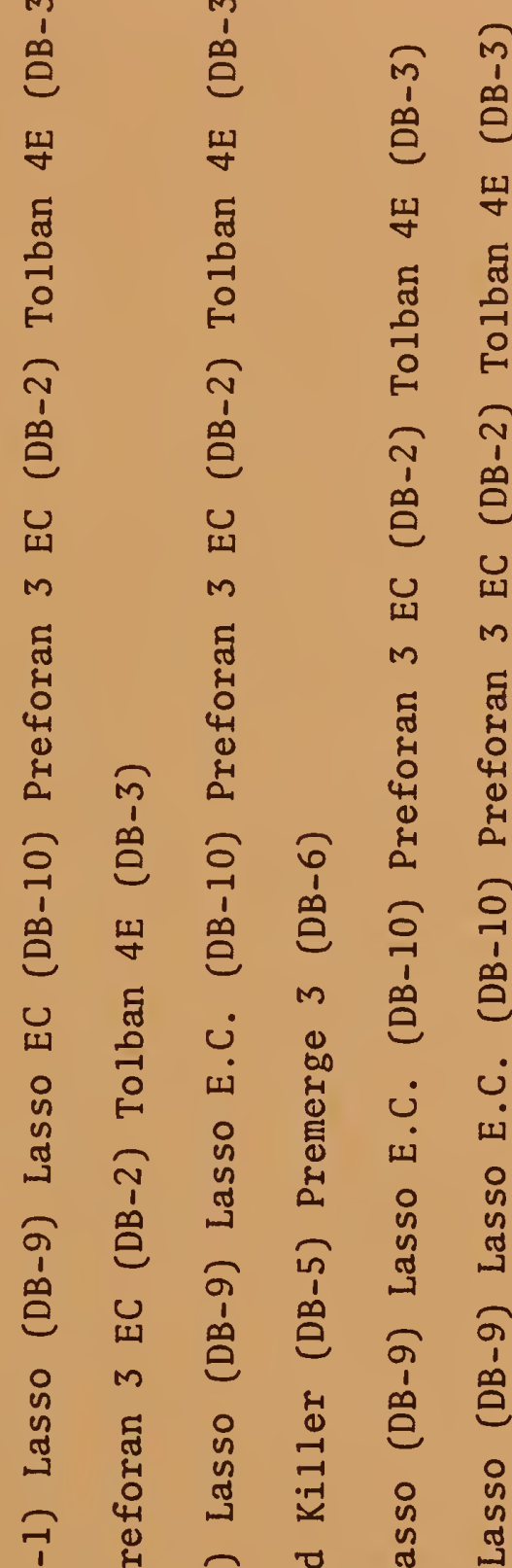

a

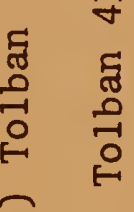

气ิ

势

鄠

क्ष

ఏे

e

政

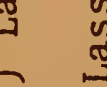

仓ิ

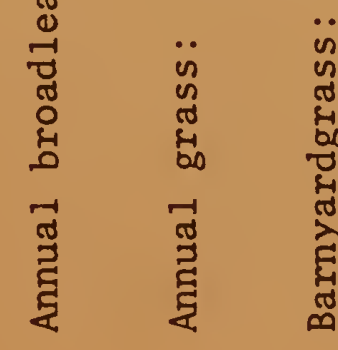

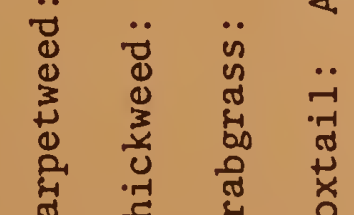
家

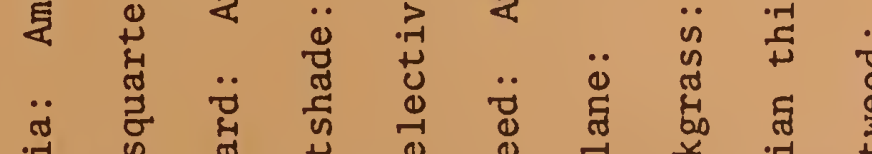

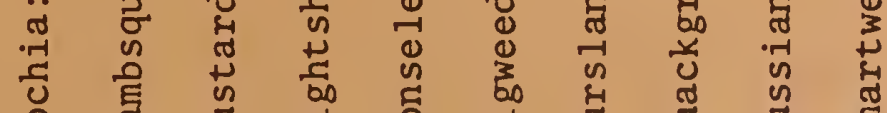




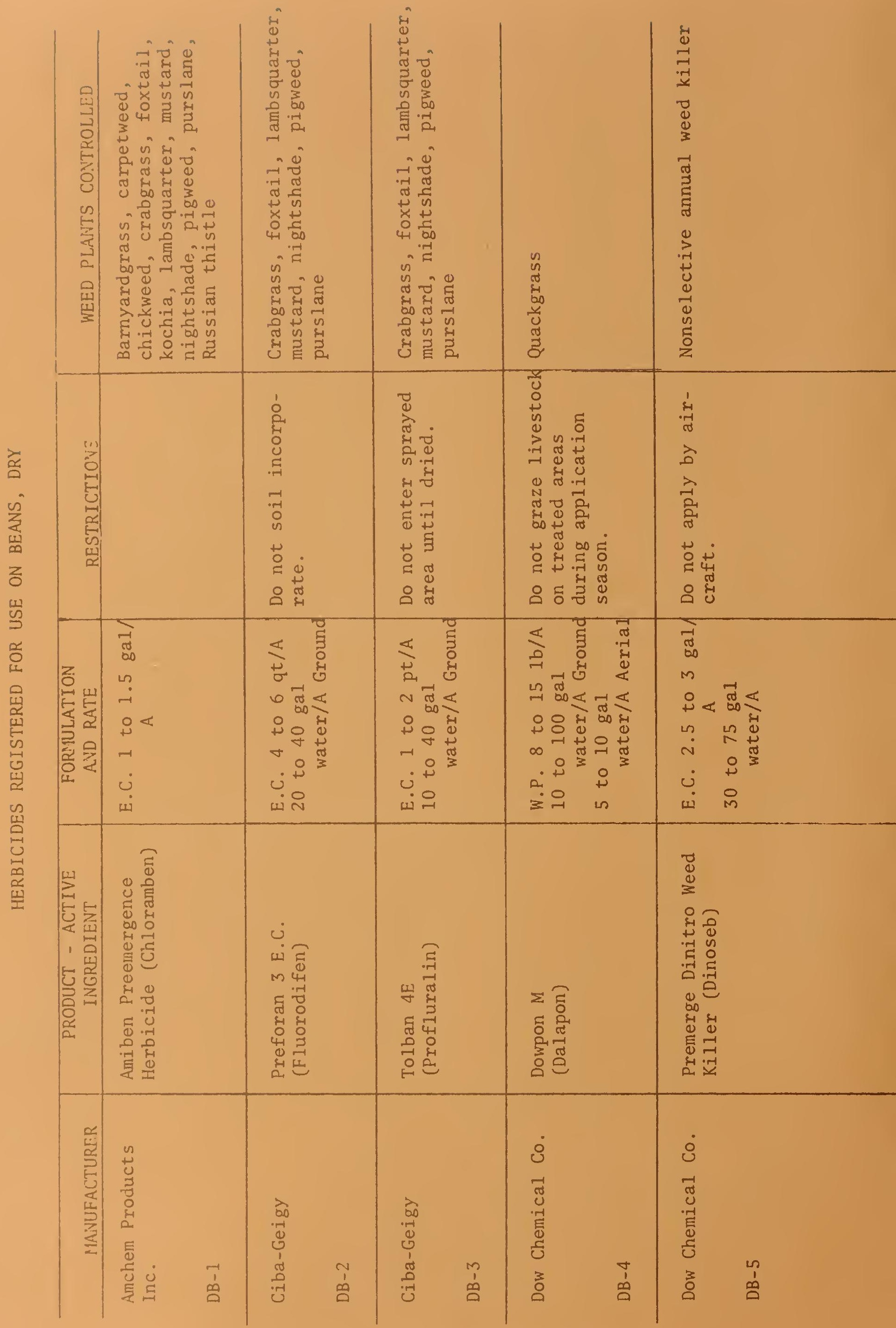




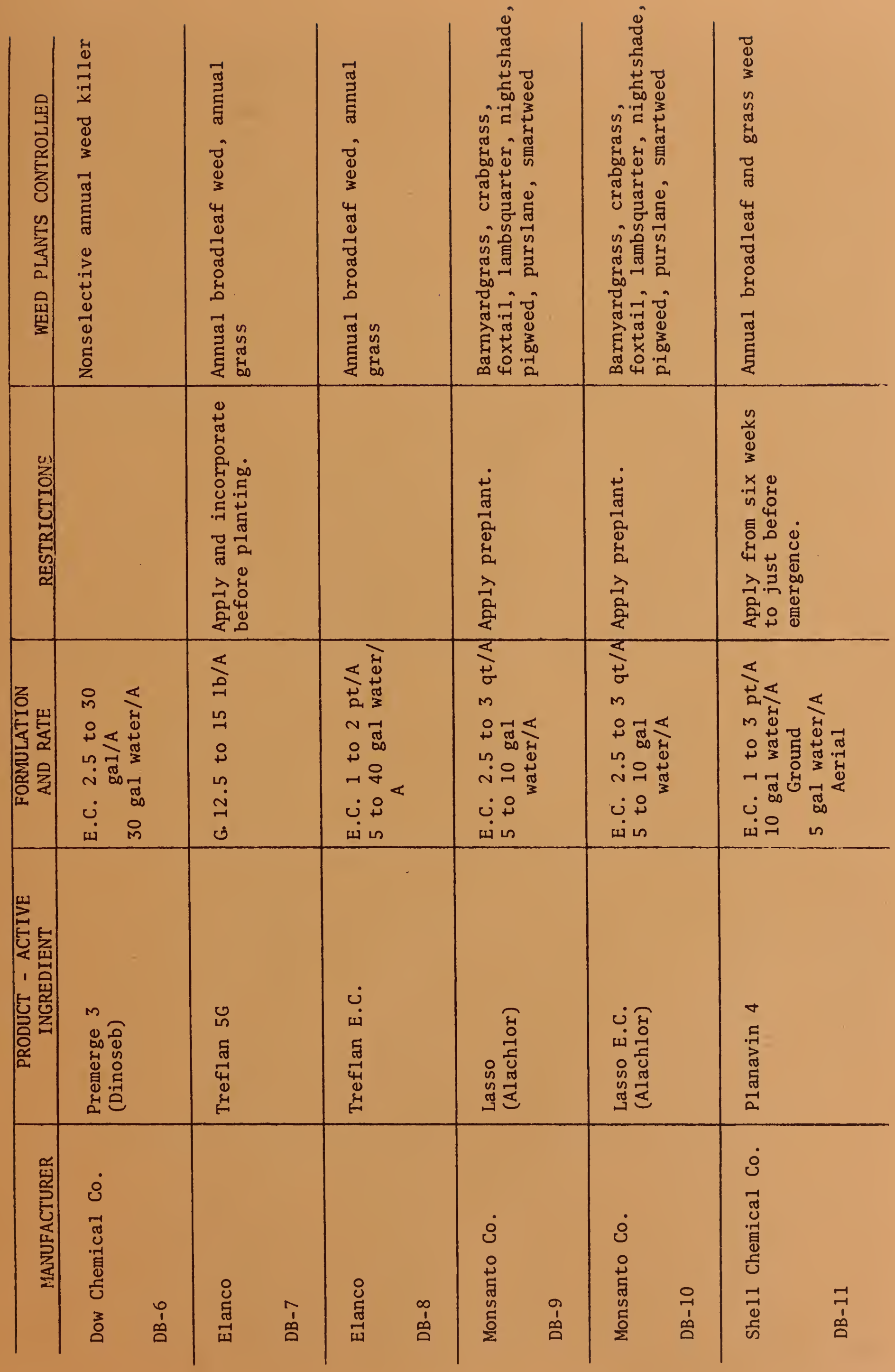




\begin{tabular}{|c|c|c|c|c|}
\hline 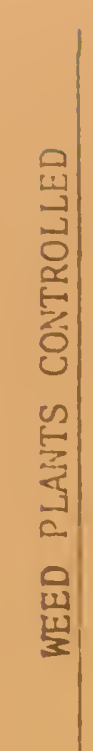 & 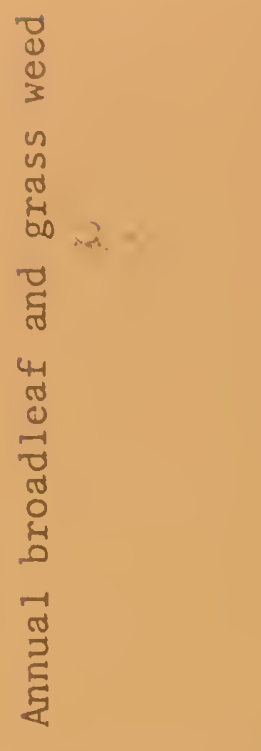 & 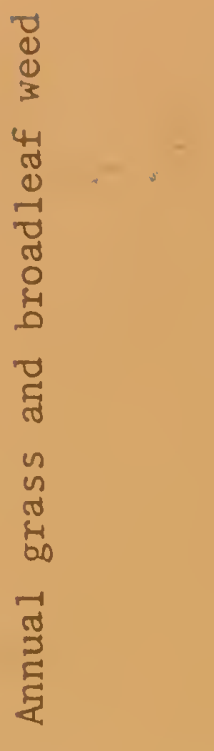 & 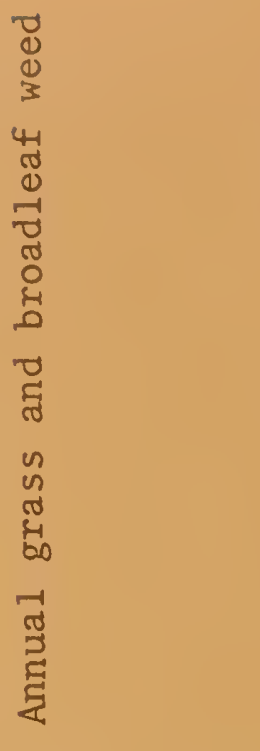 & 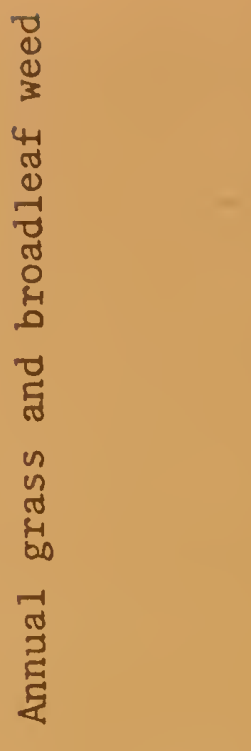 \\
\hline 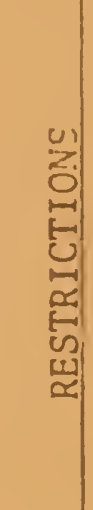 & 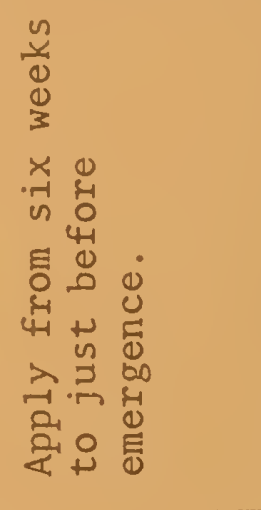 & 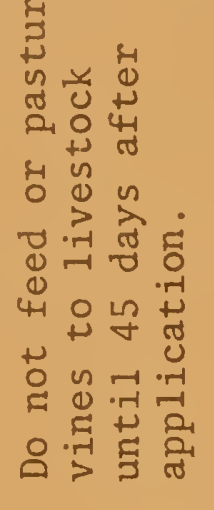 & 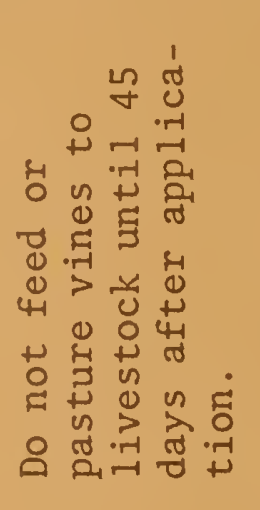 & \\
\hline 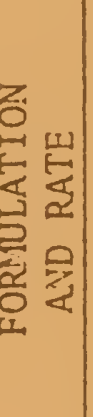 & 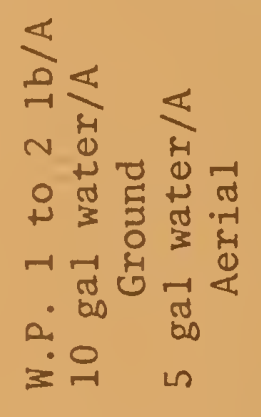 & 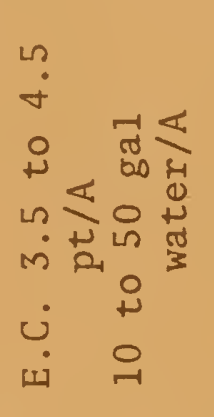 & 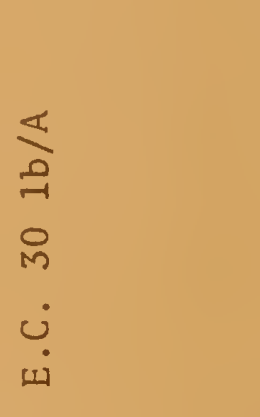 & 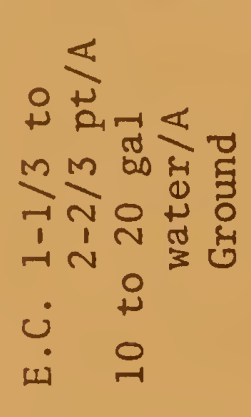 \\
\hline 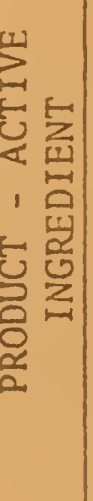 & 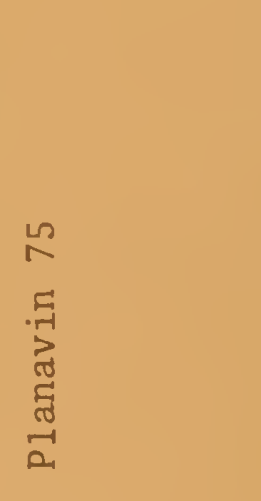 & 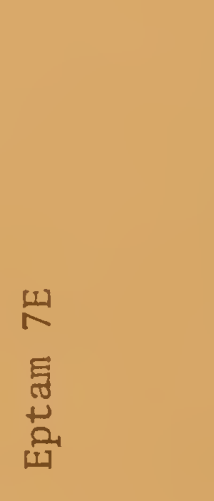 & 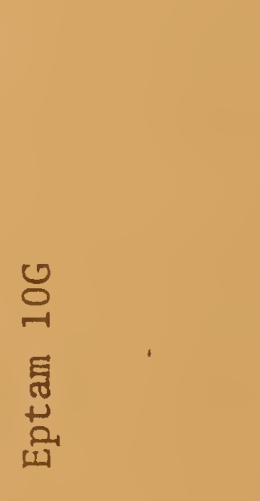 & 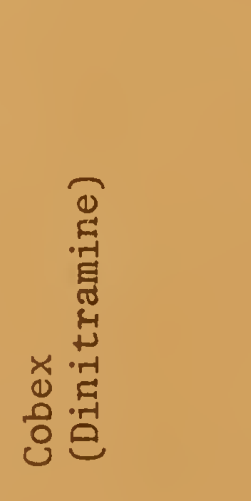 \\
\hline 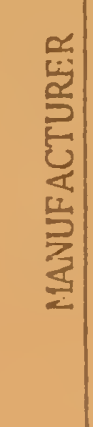 & 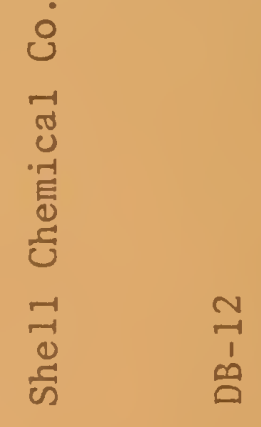 & 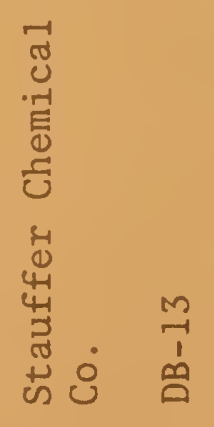 & 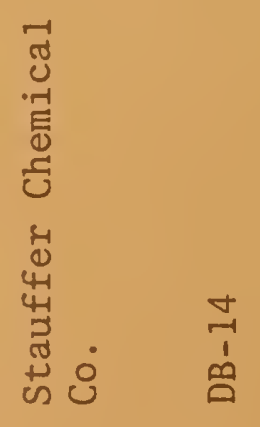 & 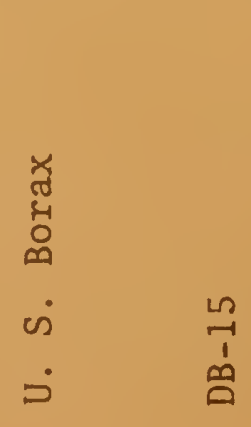 \\
\hline
\end{tabular}




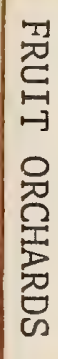




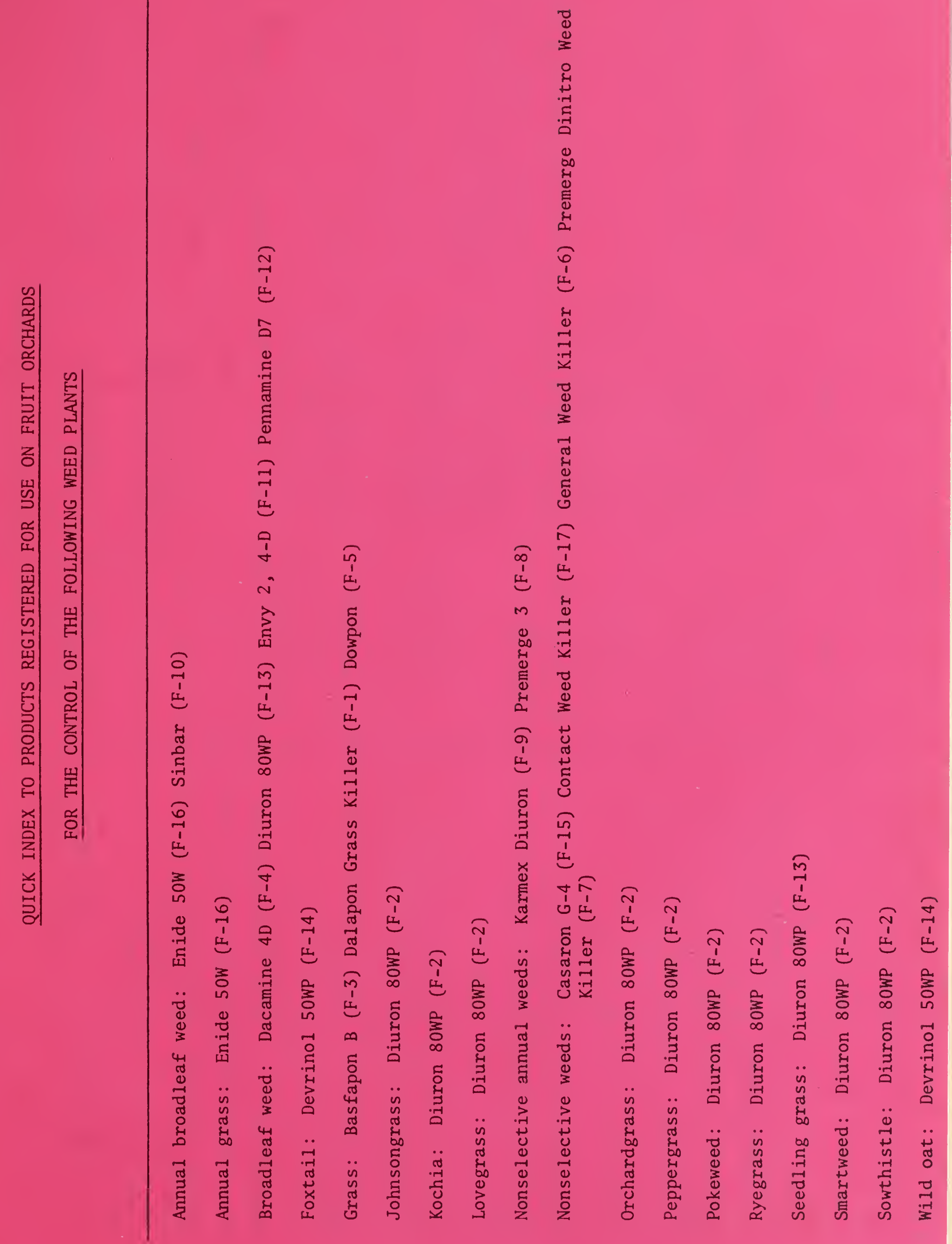


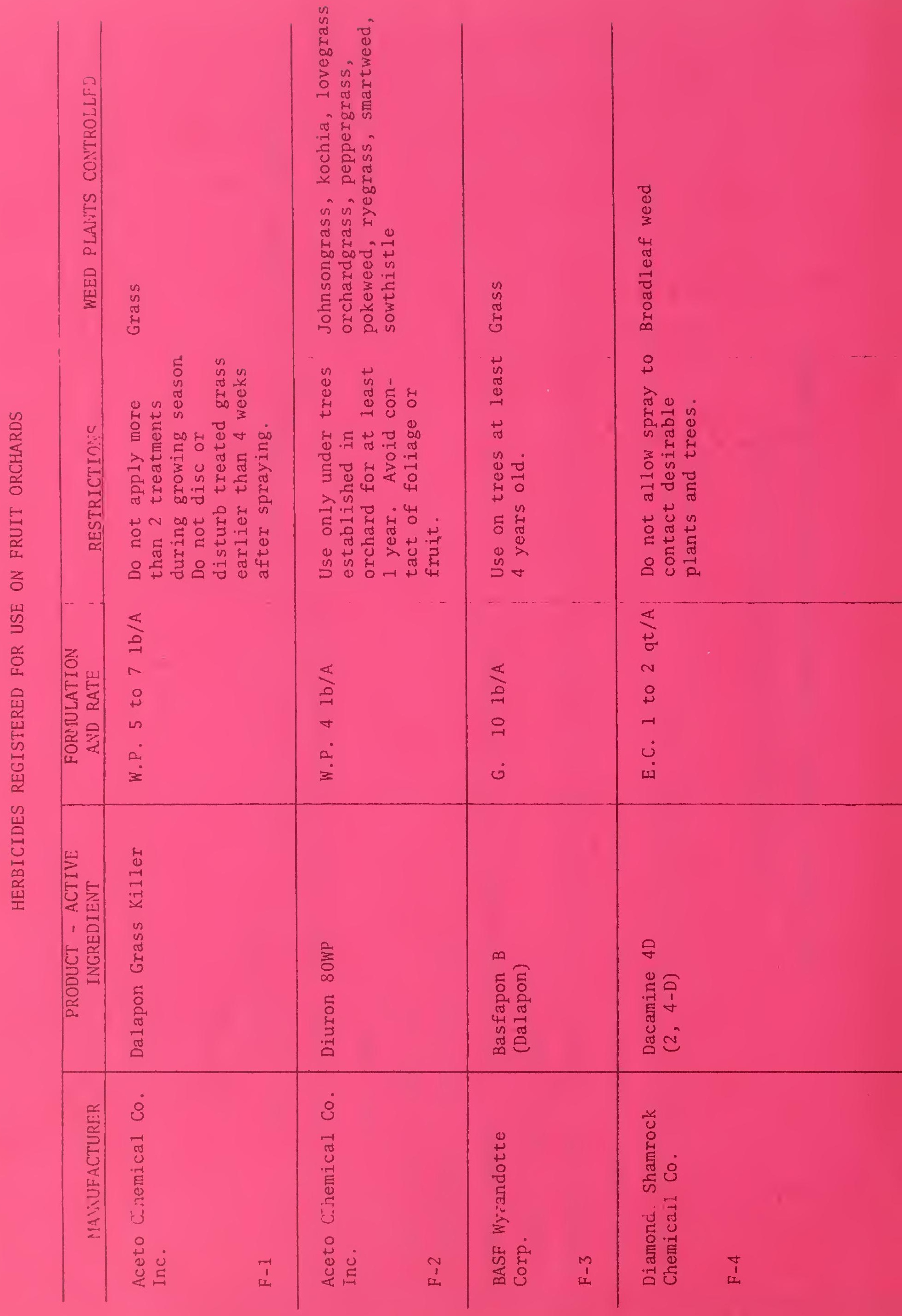




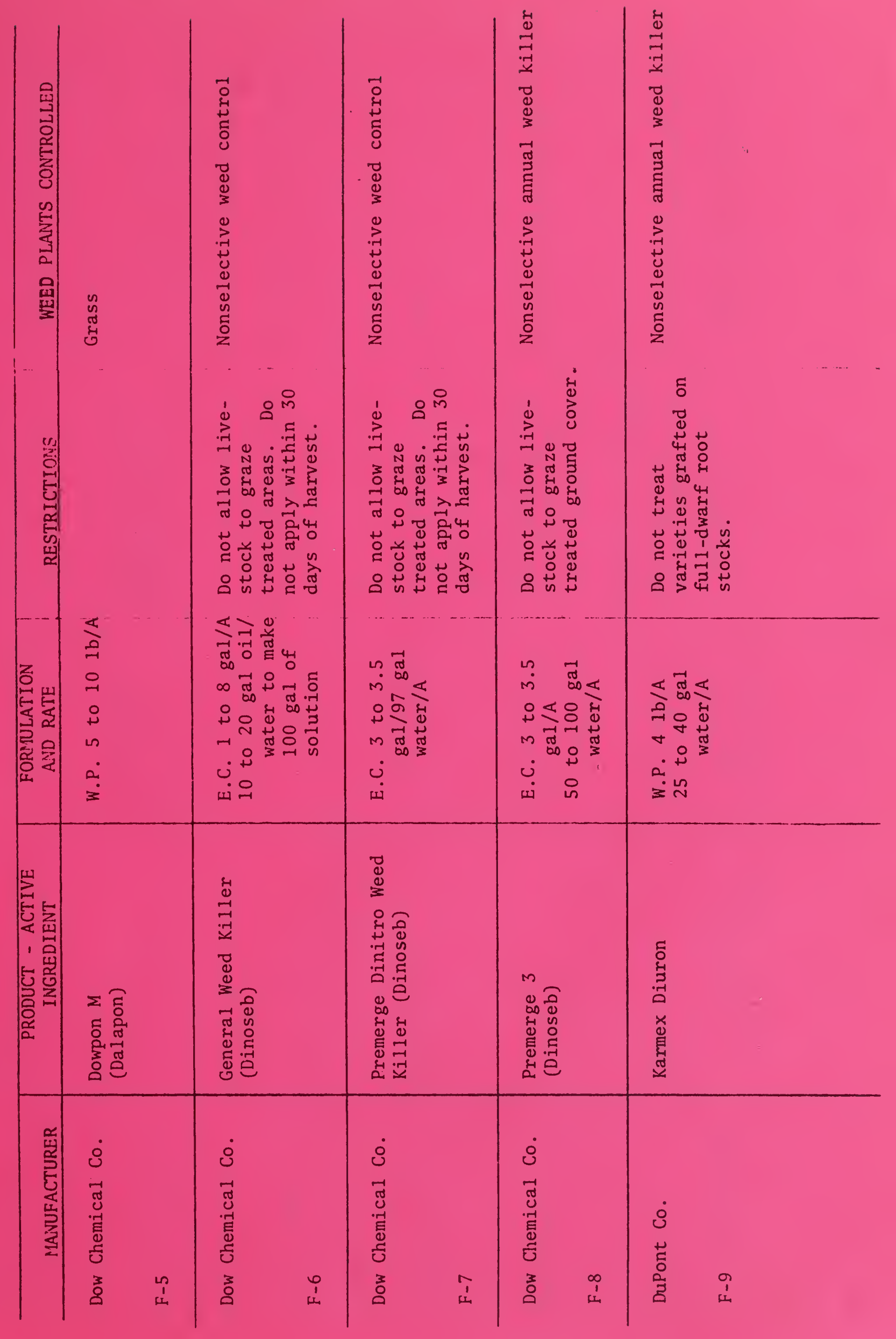




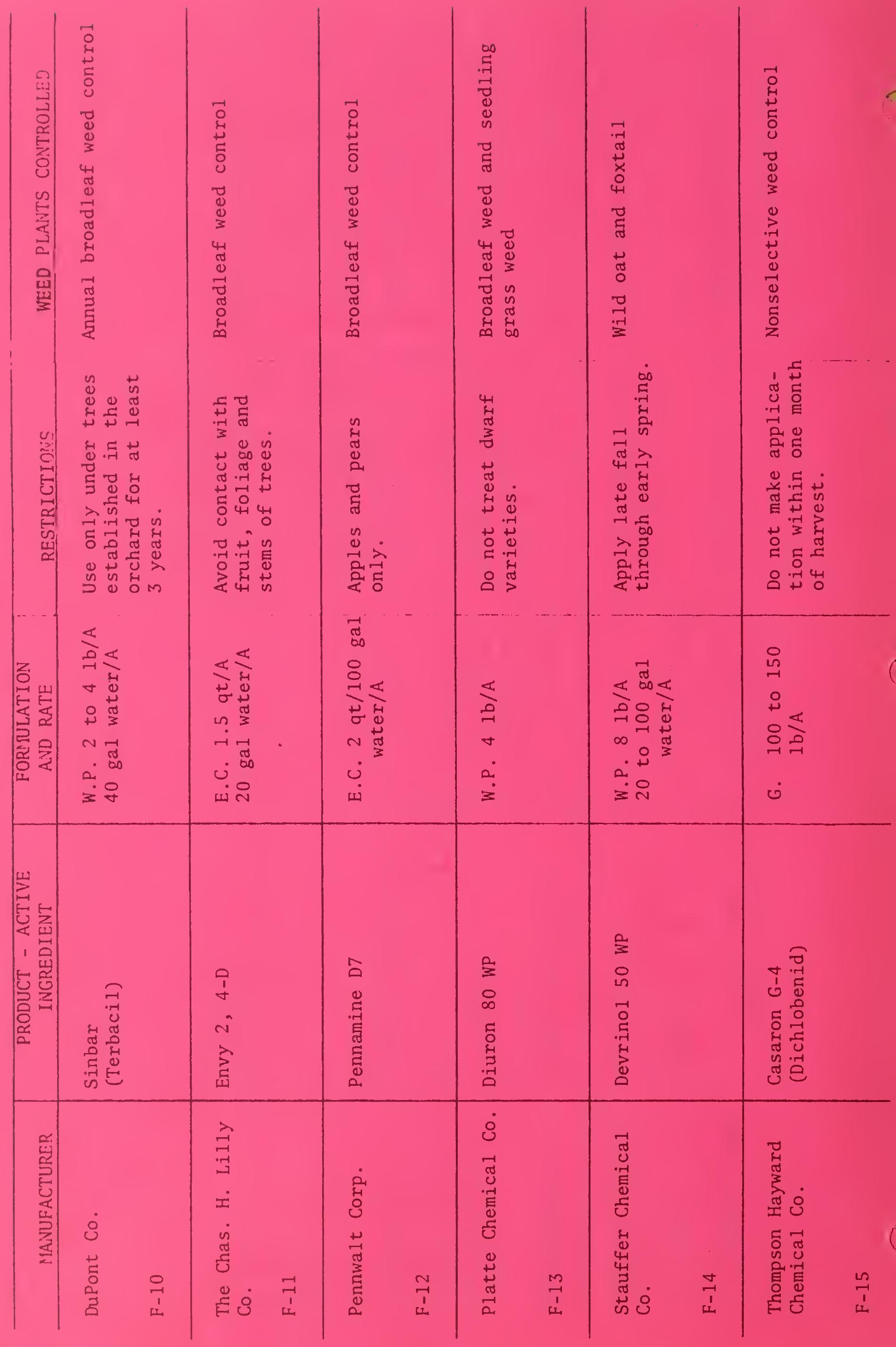




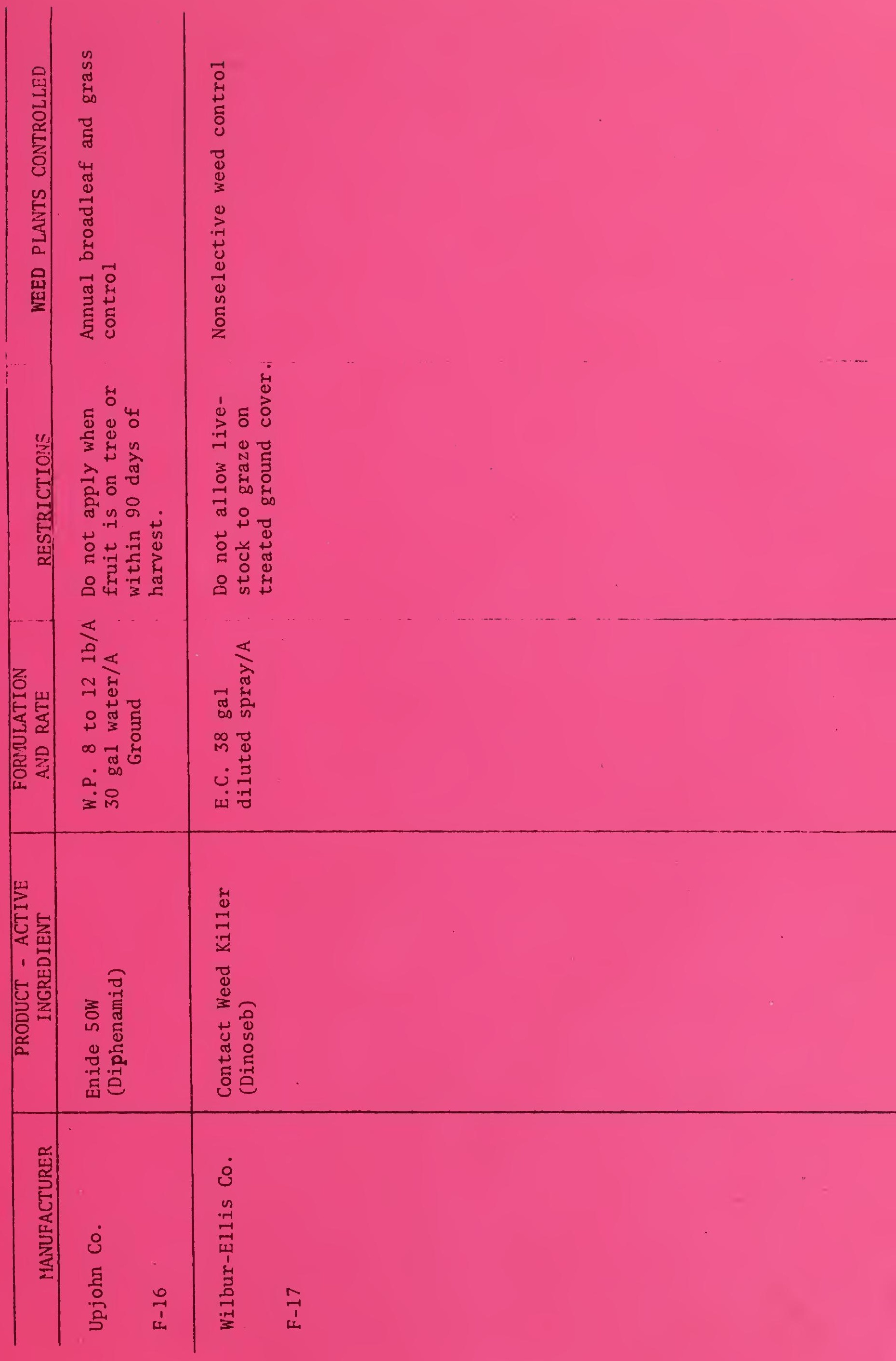





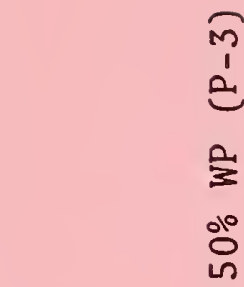

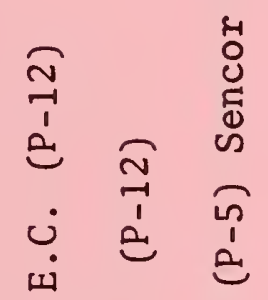

岳

总 蛋

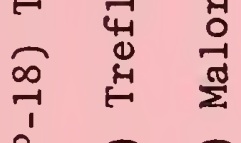

$\begin{array}{lll}2 & 0 & 0 \\ 0 & 1 & 1 \\ 0 & 2 & a\end{array}$

氙

兽

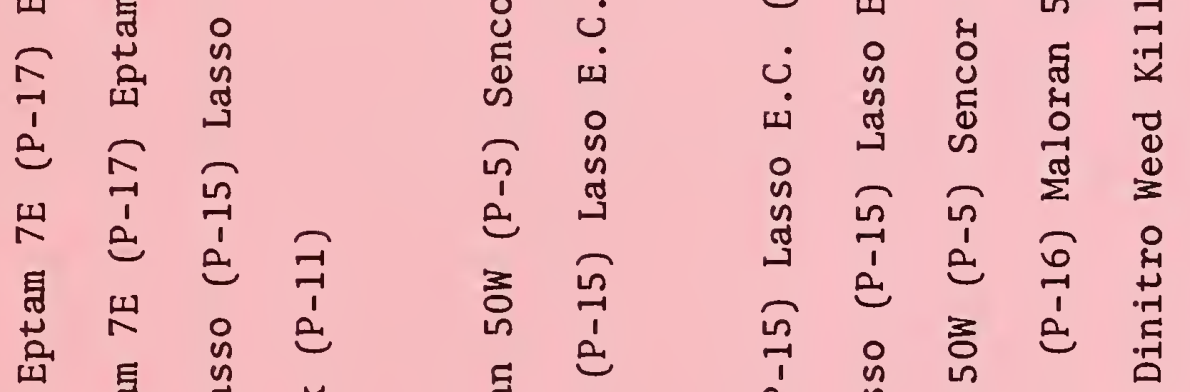

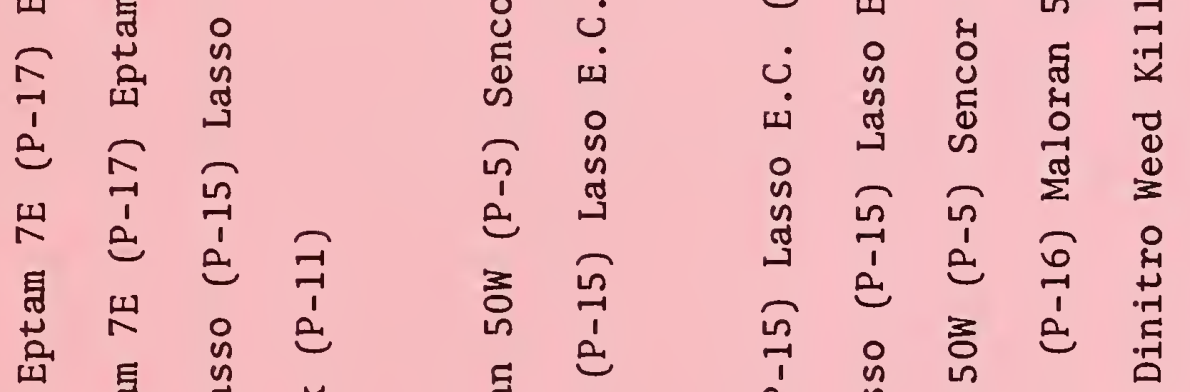

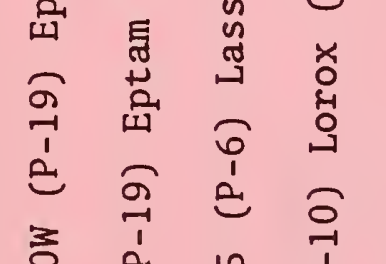

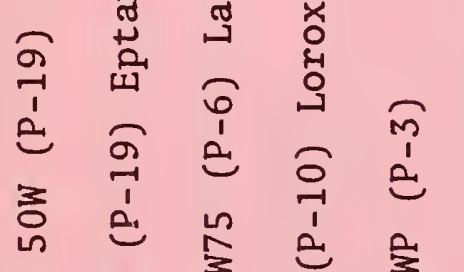

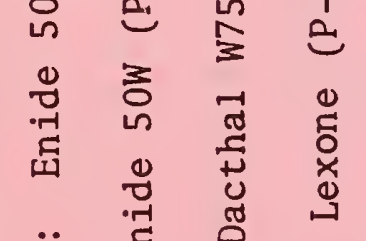

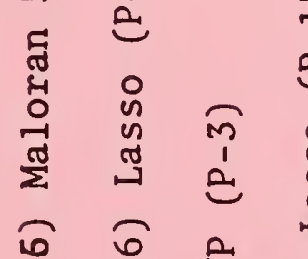

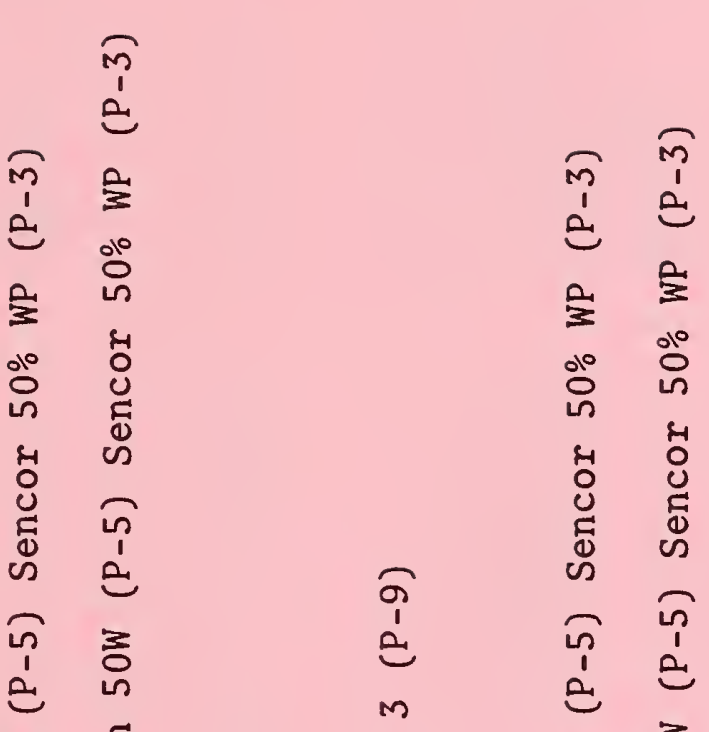

o f z z

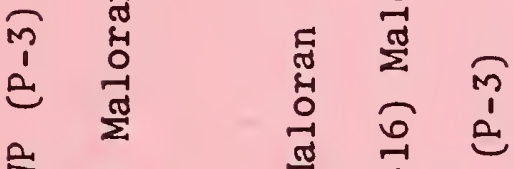

ப)

更

대용

(2)

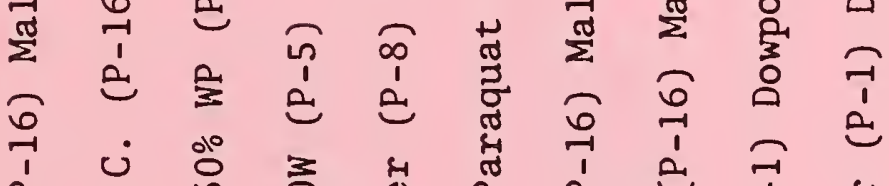

อ फ के

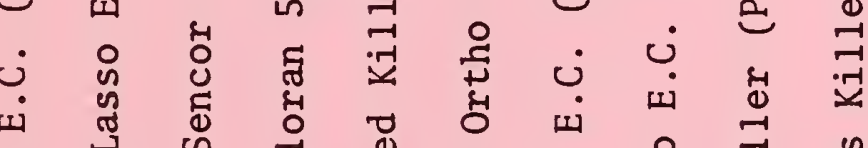

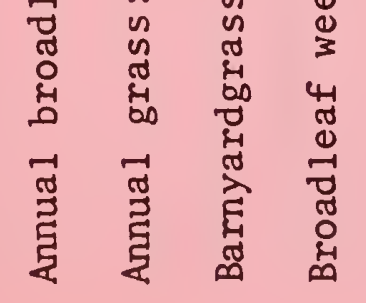

$\ddot{\varpi}$ 㝏

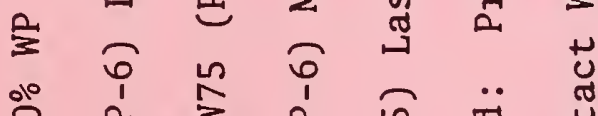

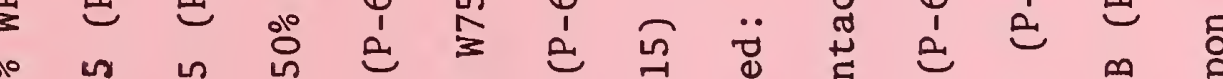




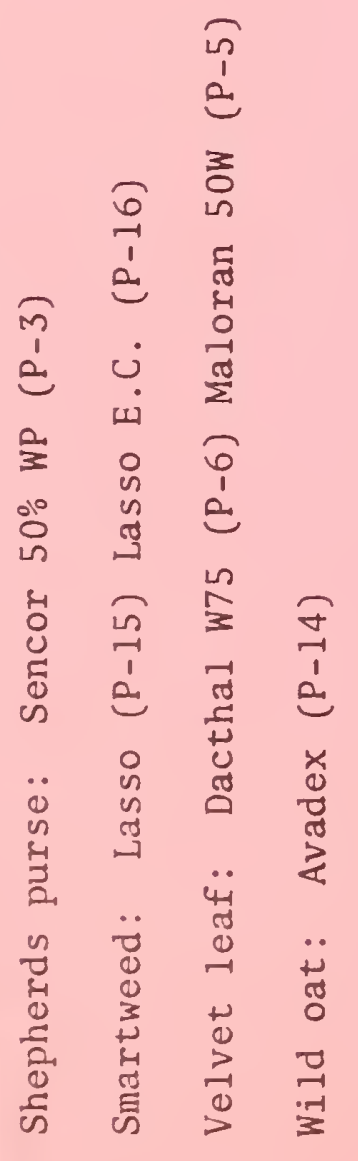




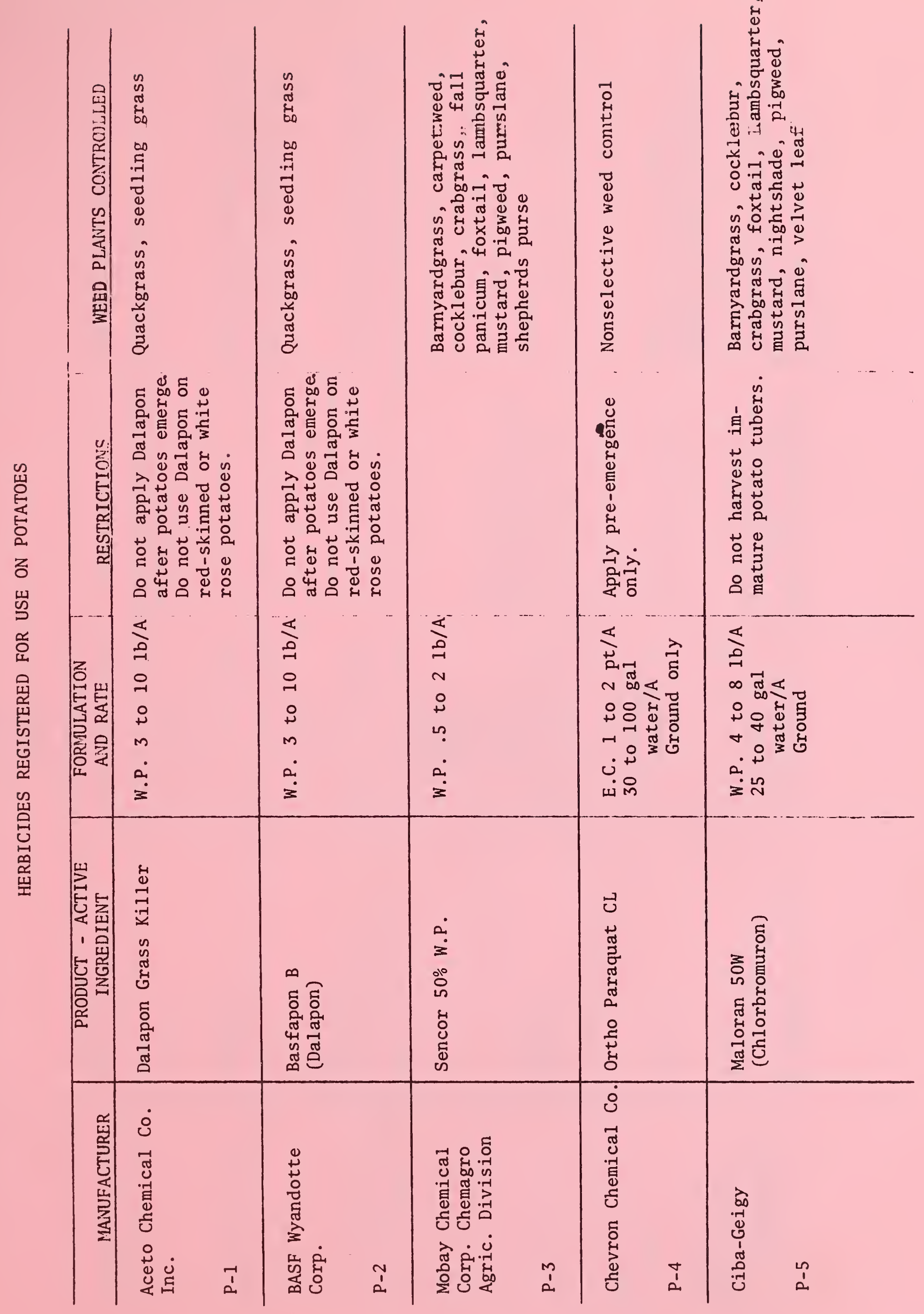




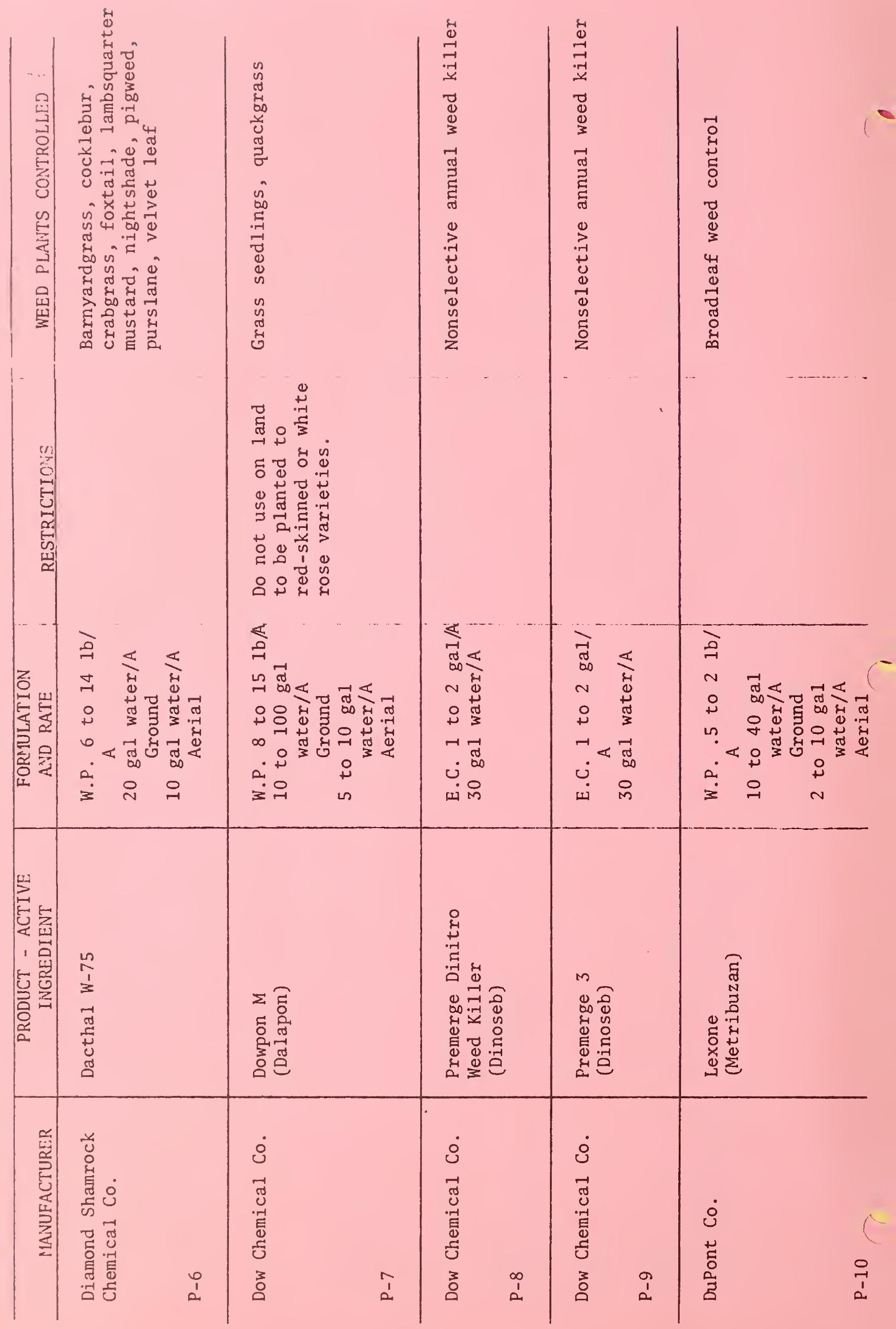




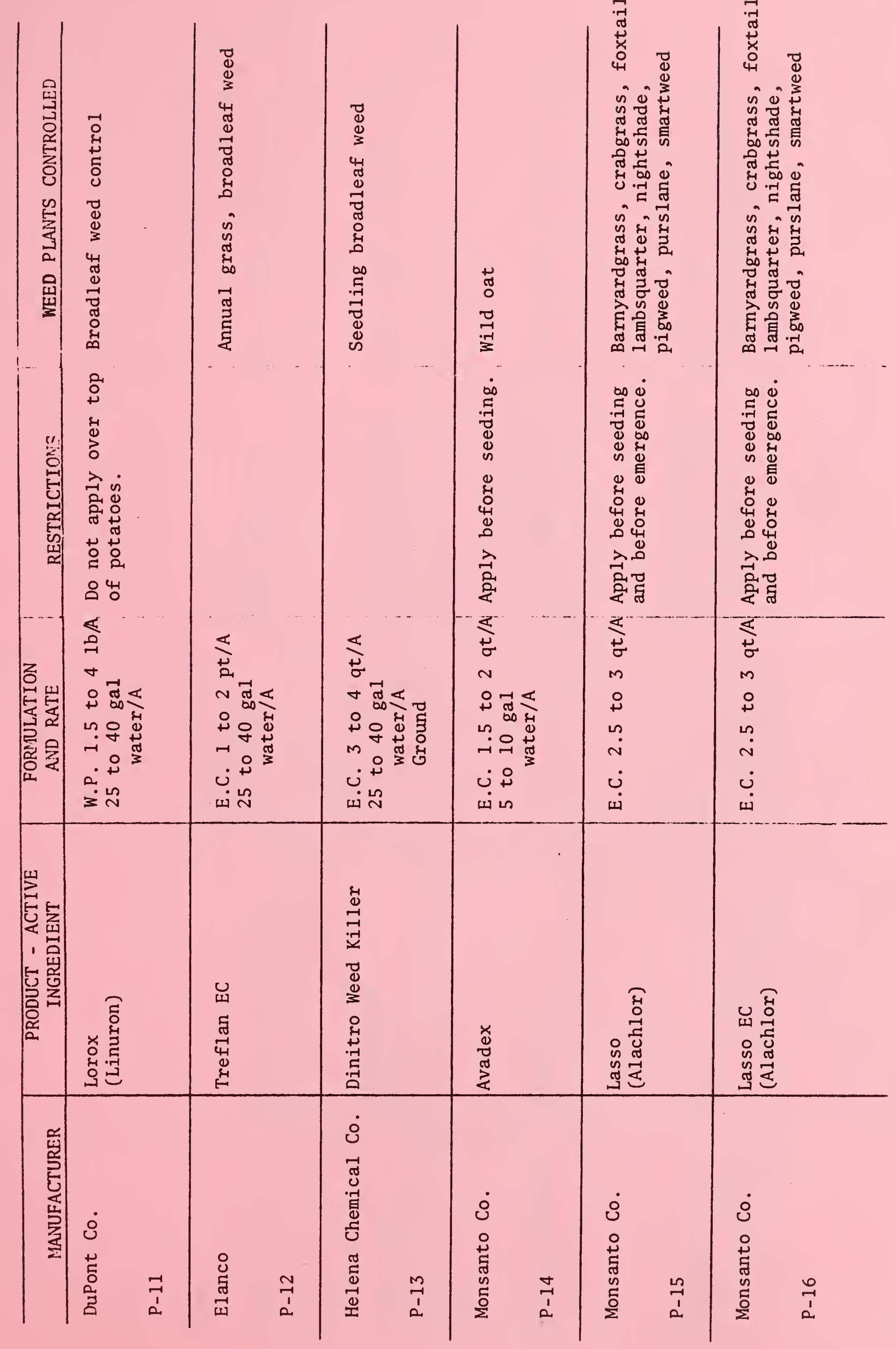




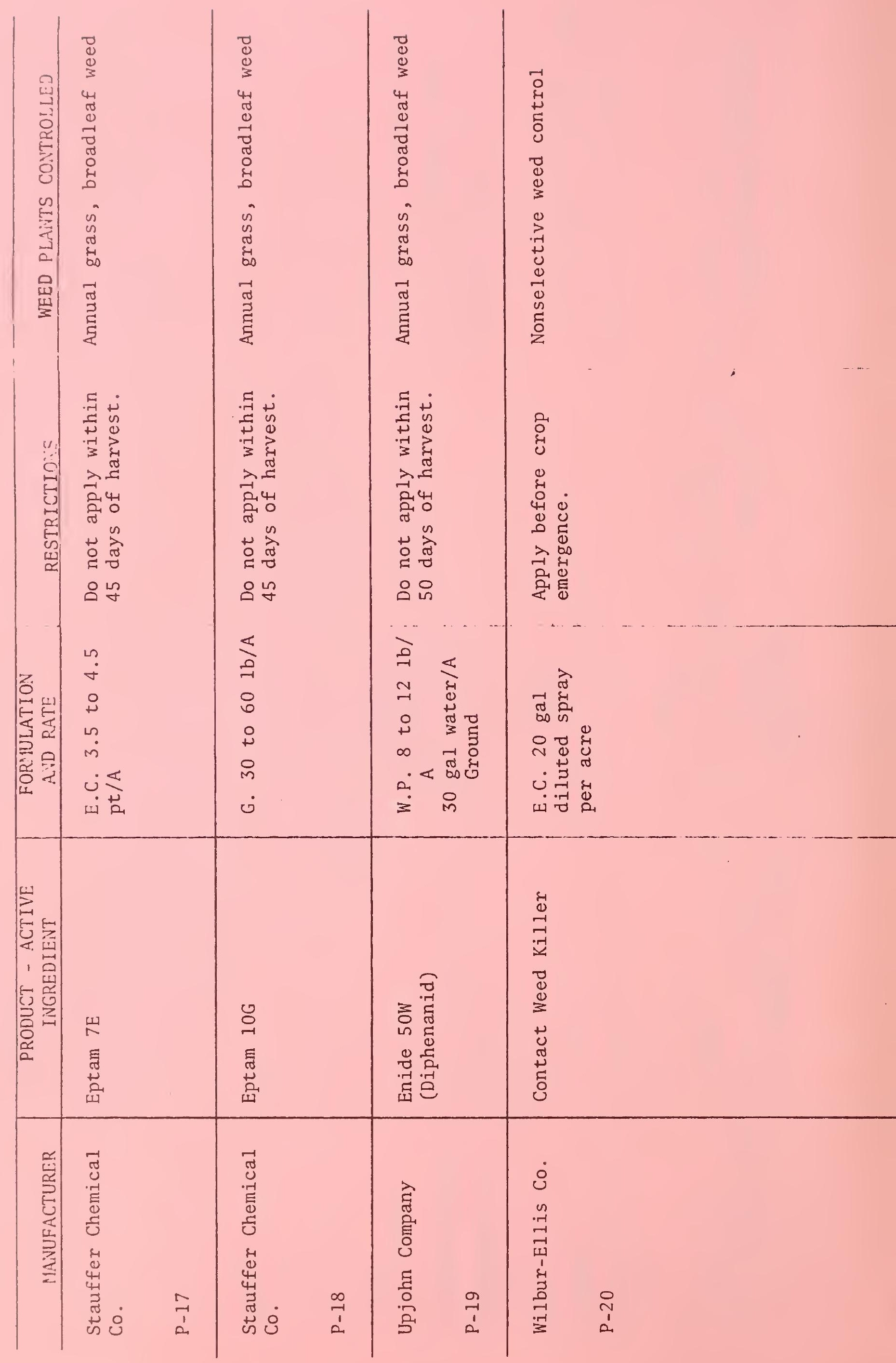





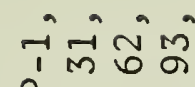

乎 용저

İ 00 in

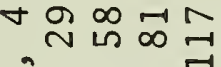

त ธก ${ }^{\infty}$ is 80

$\therefore a n$

ปñ

至

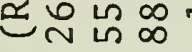

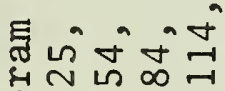

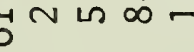

पैं⿰彳ं⿰彳

a $N$ in $\infty$

min.

ชิำ

Ni-i-

o 2 in $\infty$

तิ่ $0^{\circ} 0^{\circ}=$

次

n

$\infty \sim ⿻ \infty \infty^{\infty} \infty \infty^{\infty}$

$\infty$

ดับำ

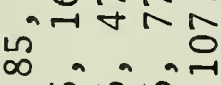

넥앙요

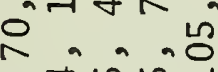

검 约

मेंतें

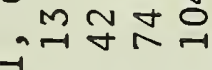

$\sim$ iñ

0

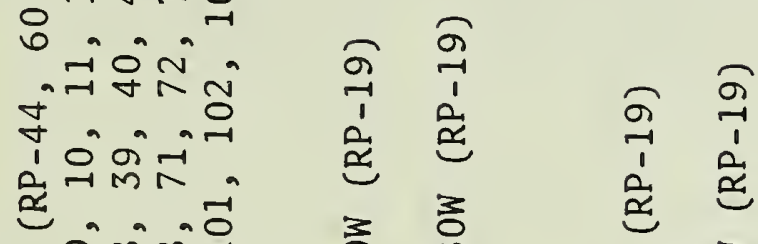

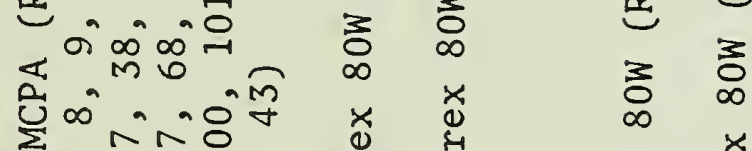

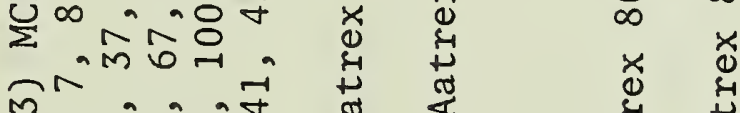

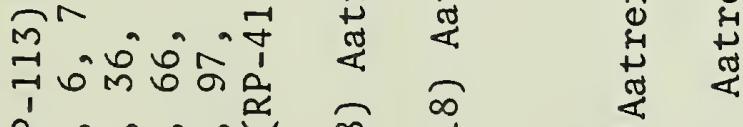

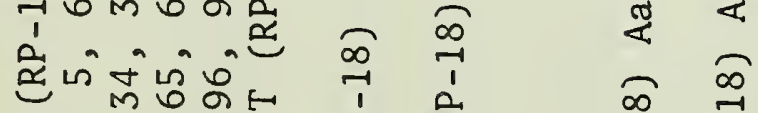

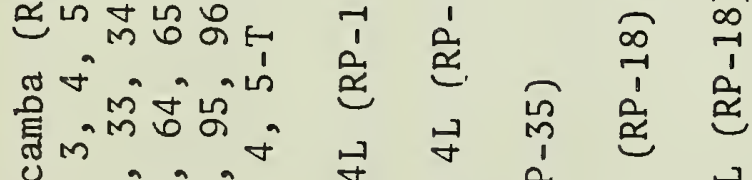

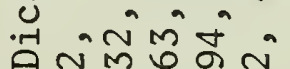

$\ddot{\vec{g}}$

अ $\times$ 品

离

4]

.

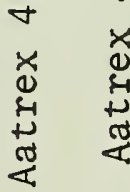




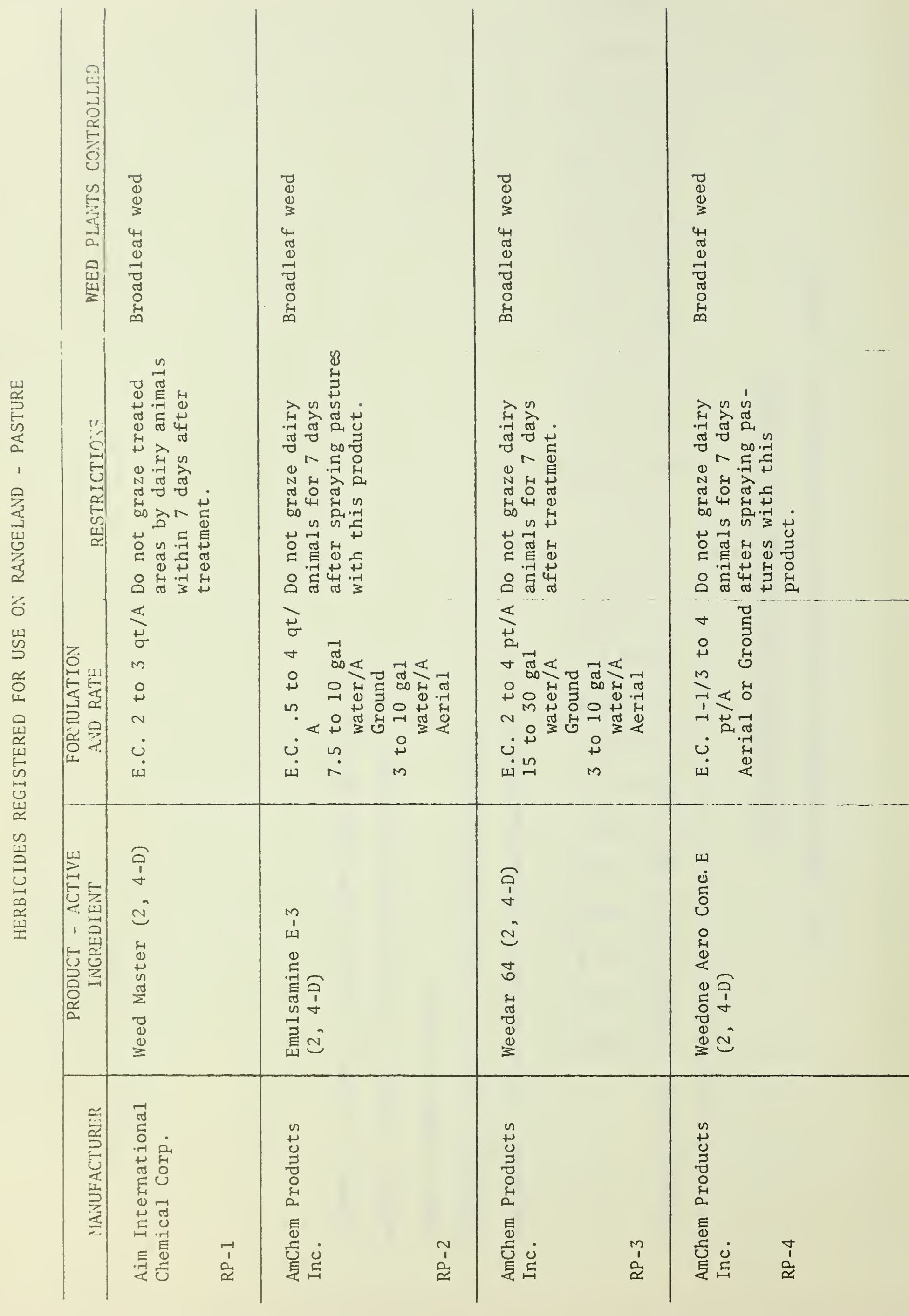




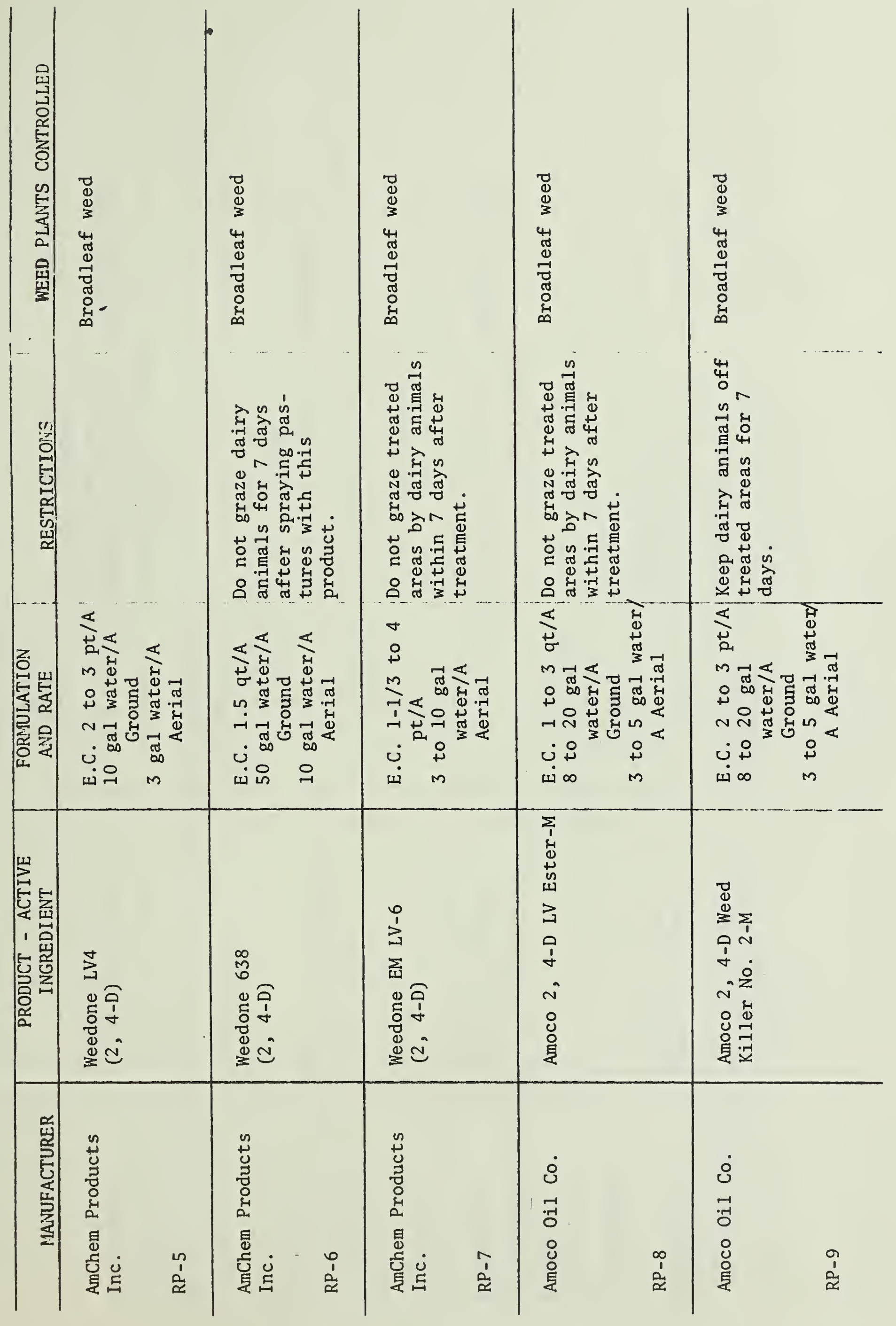




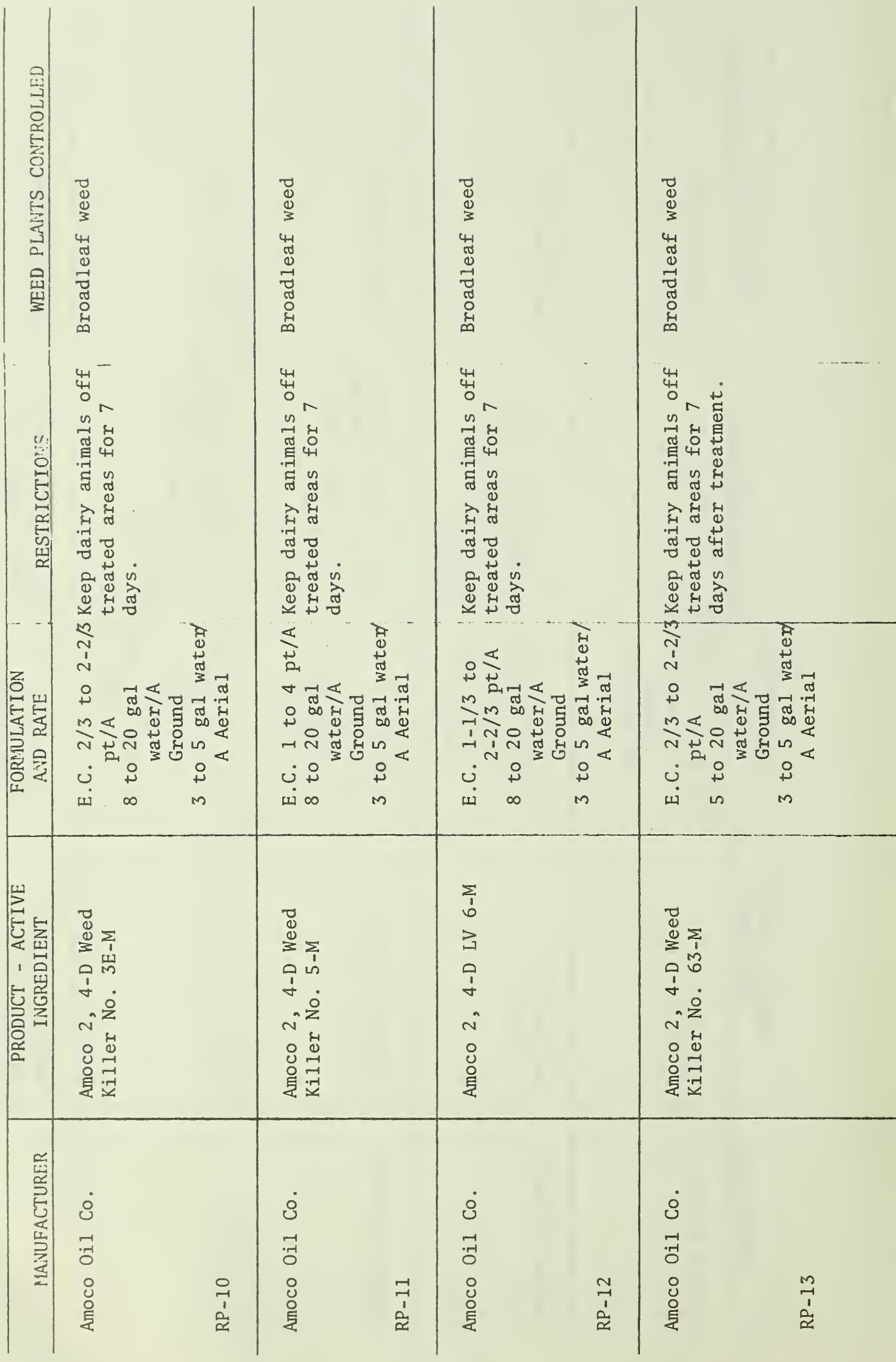




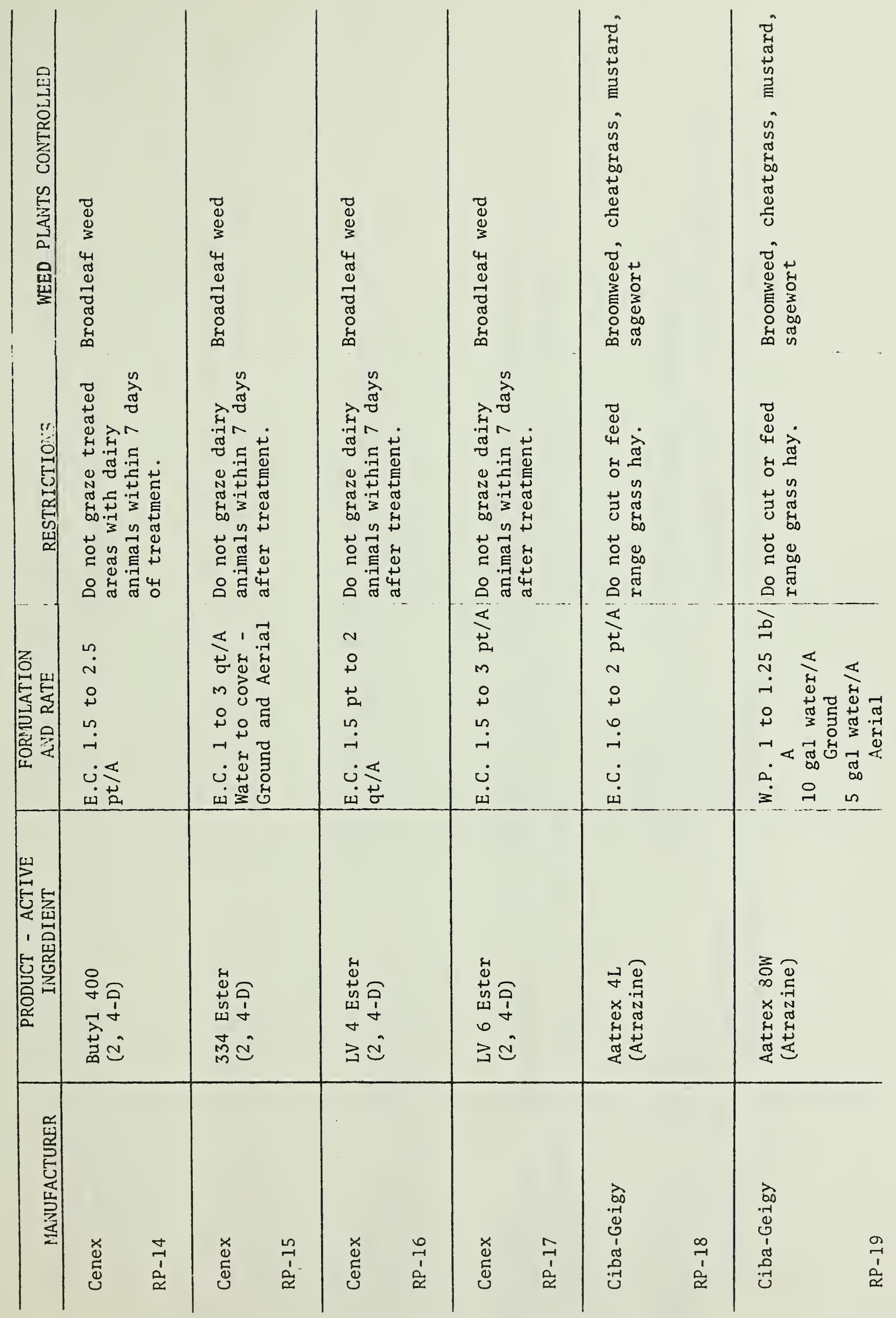




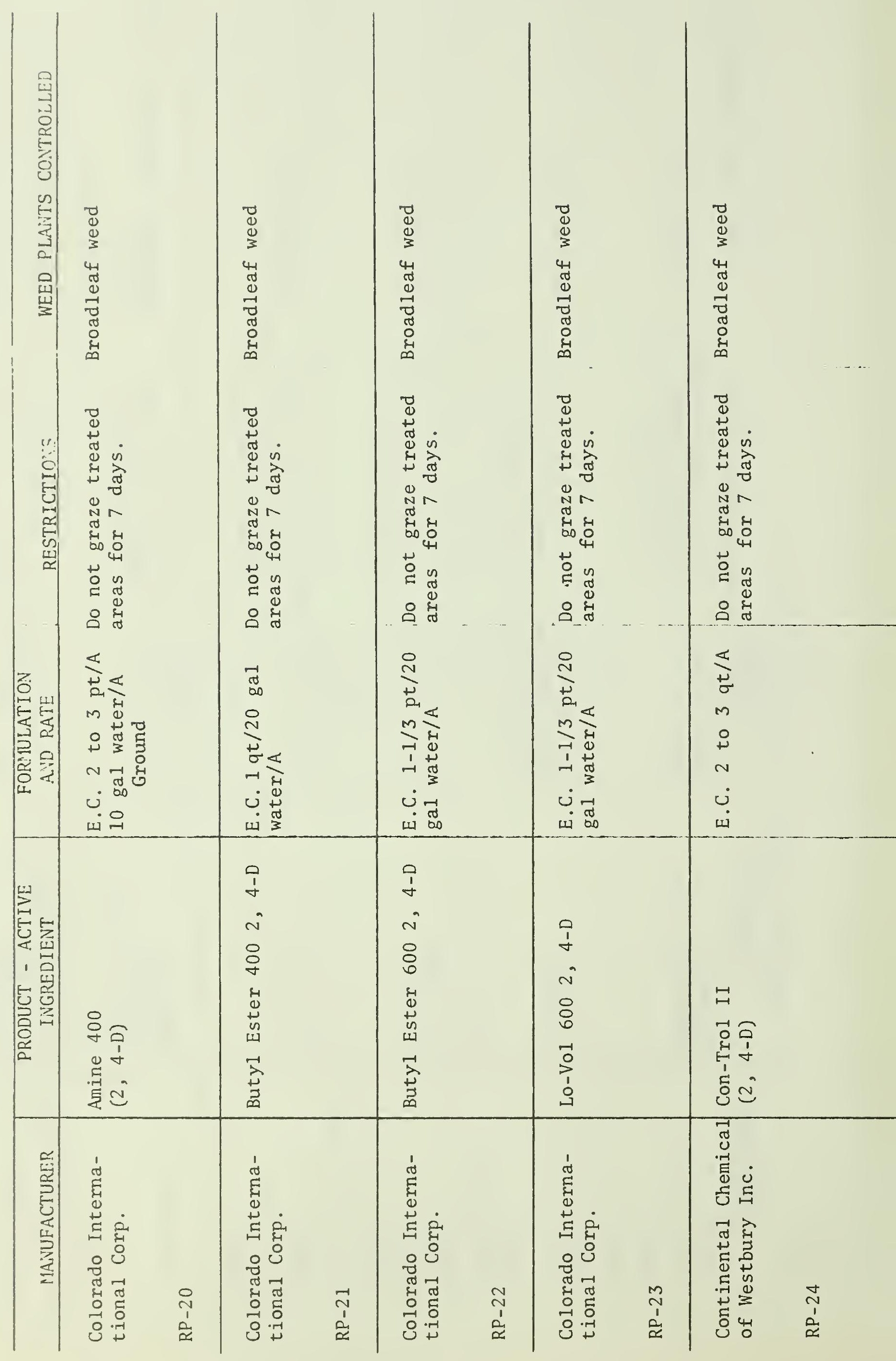




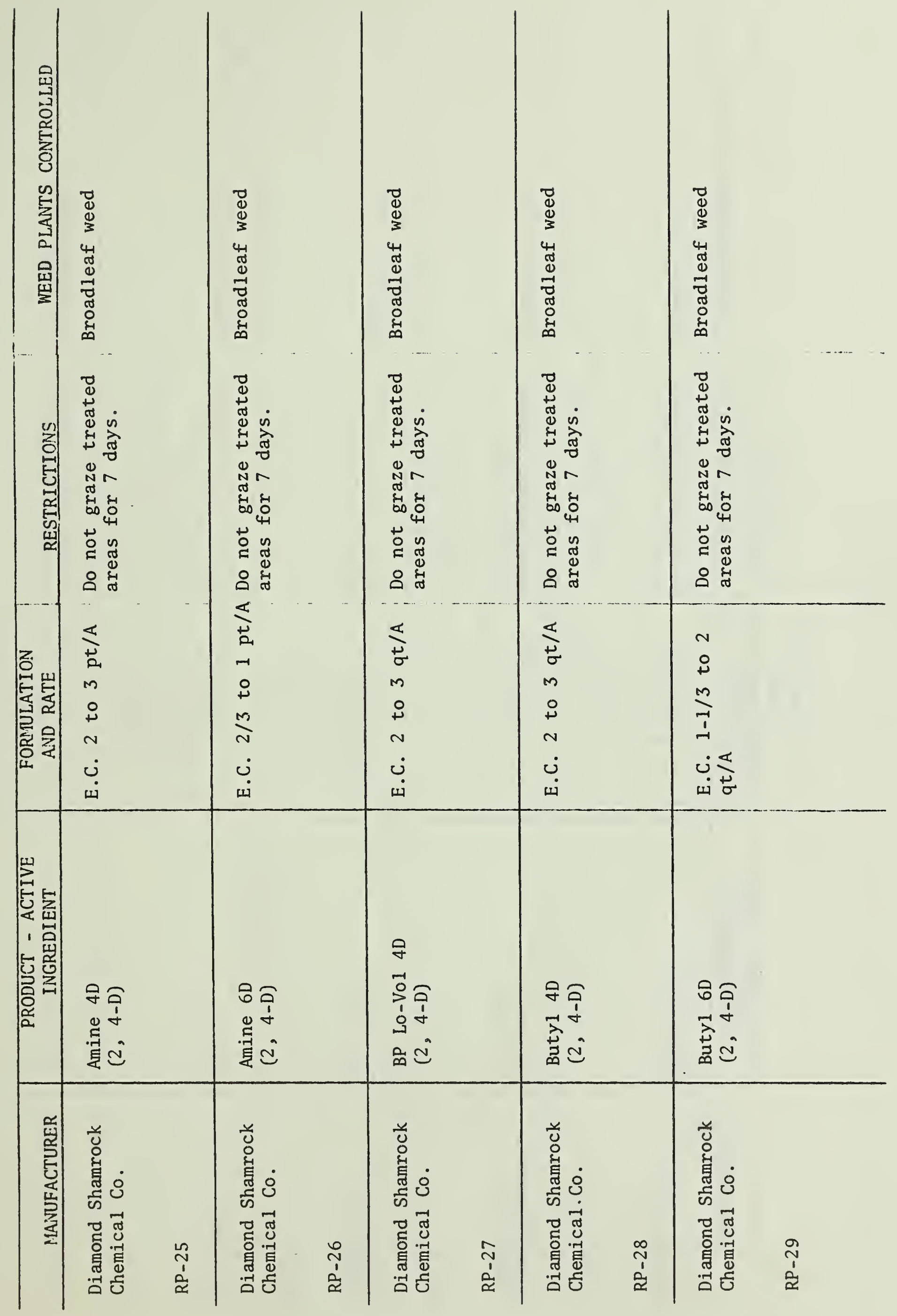




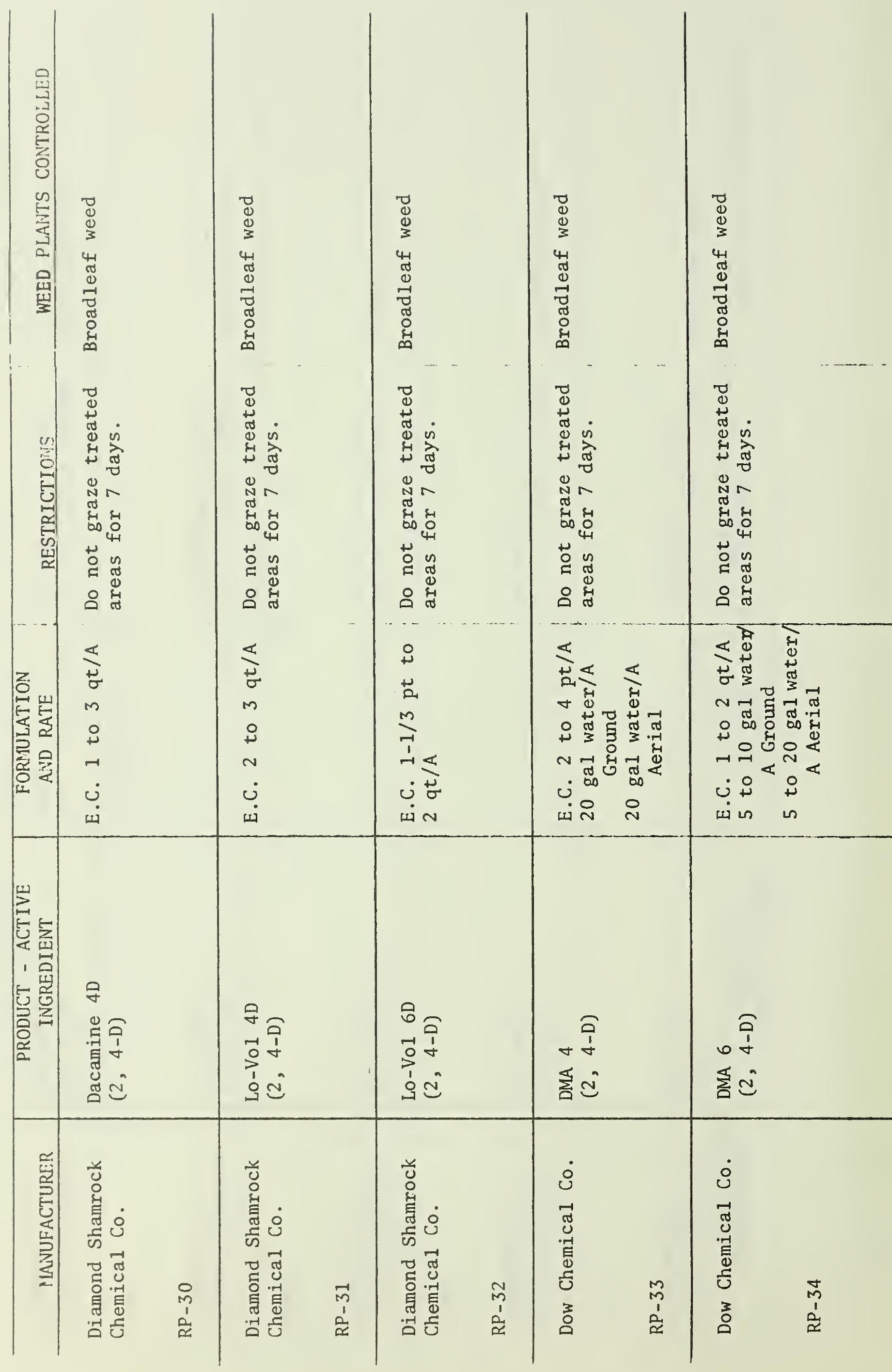




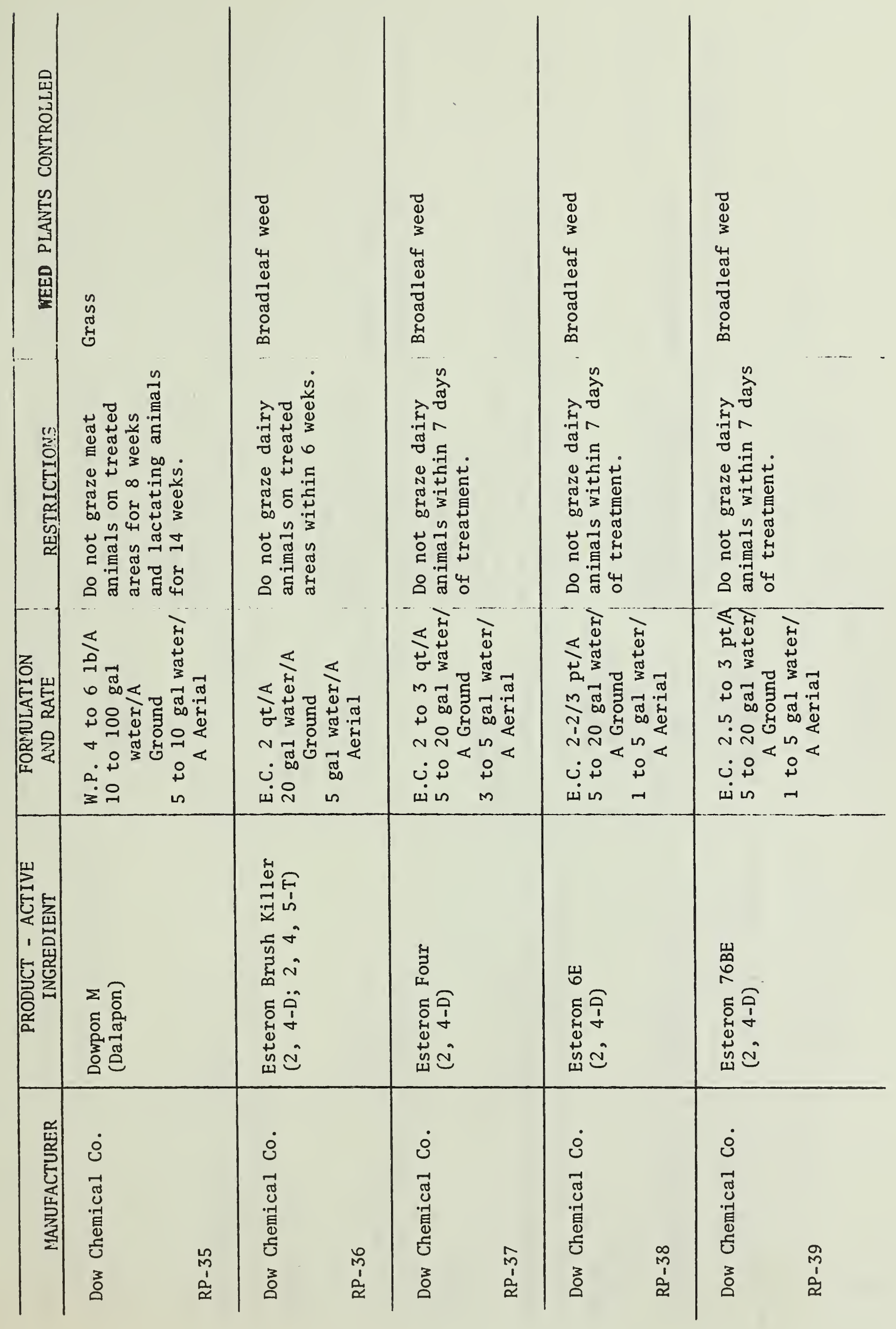




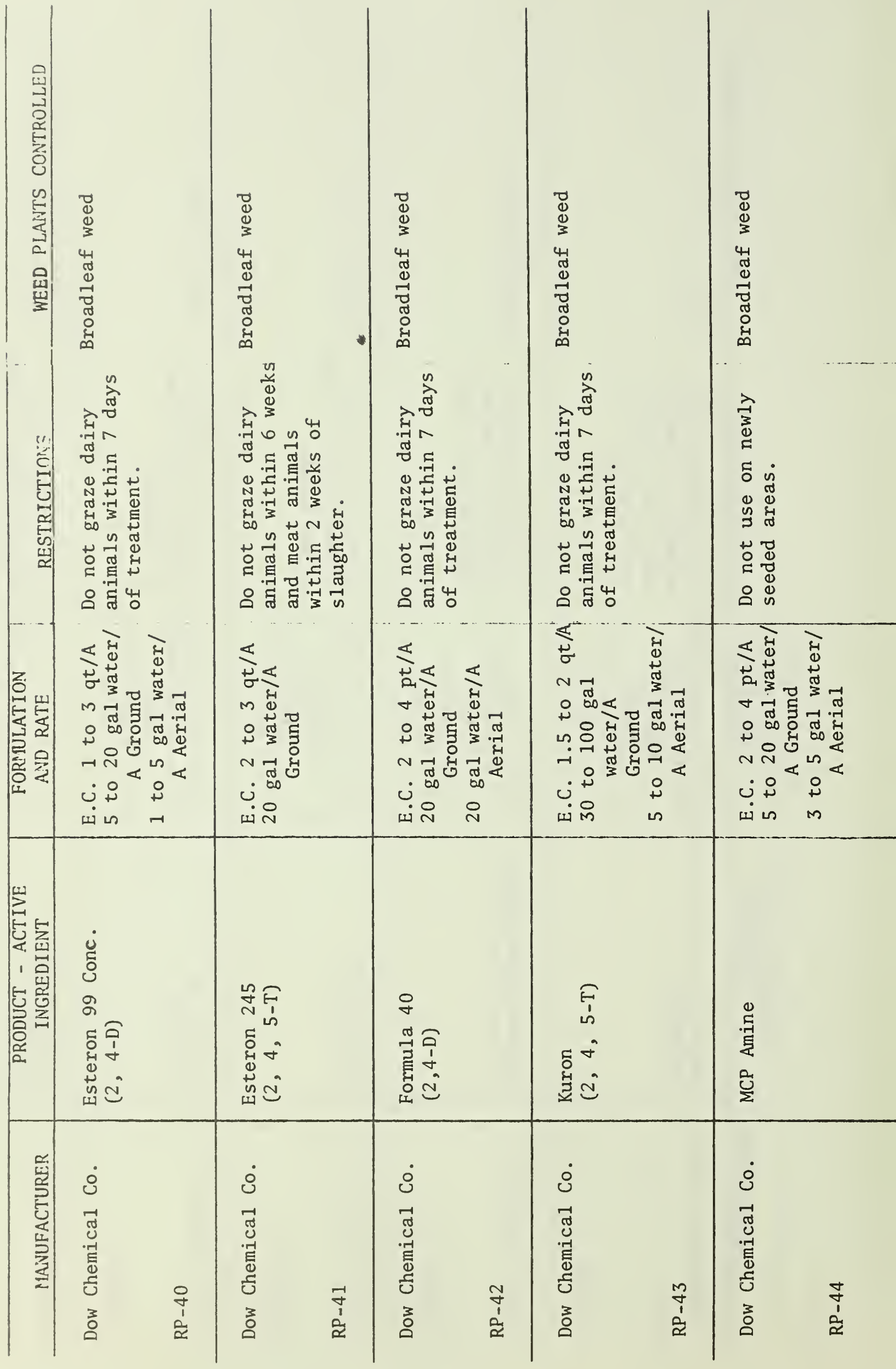




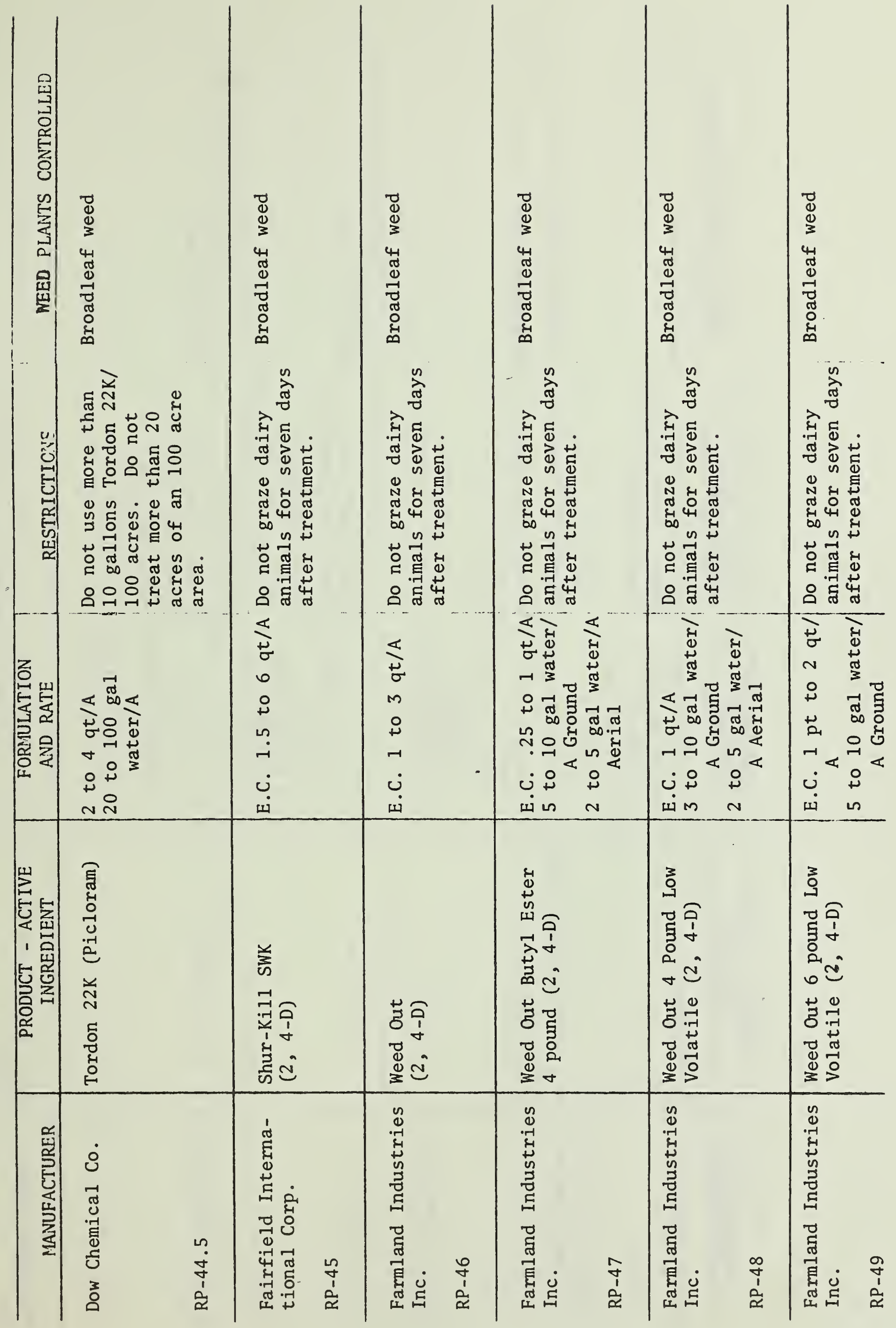




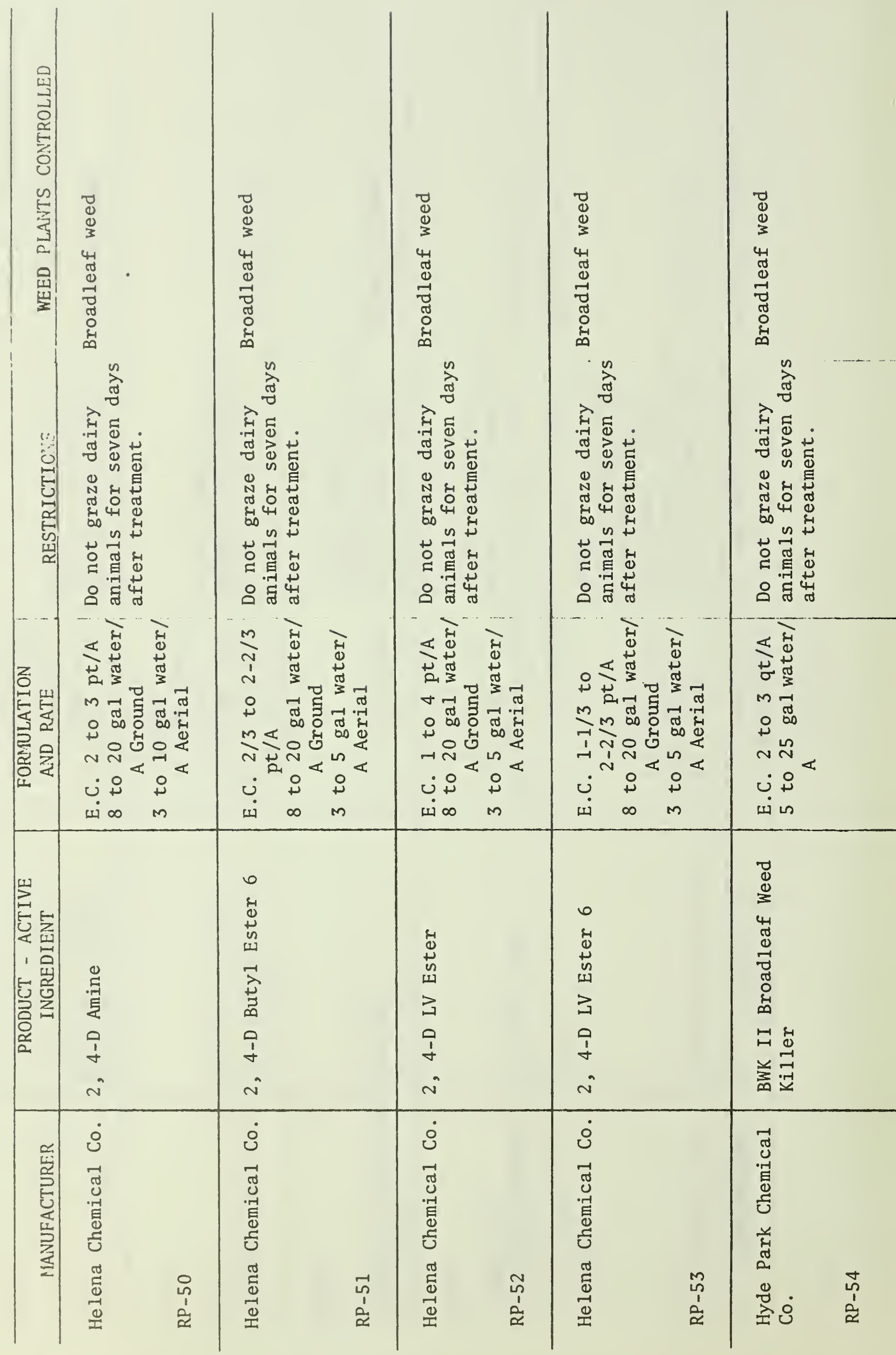




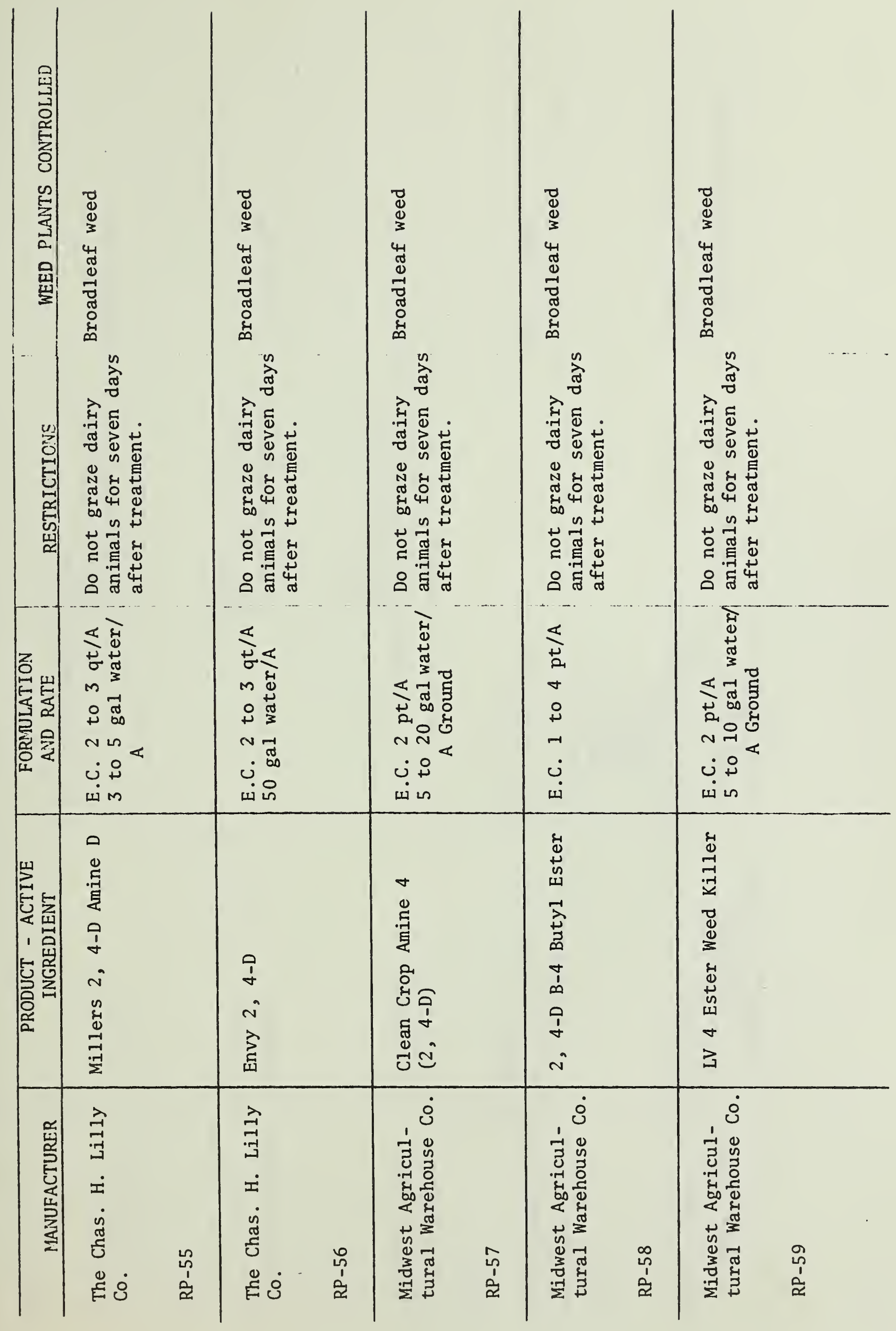




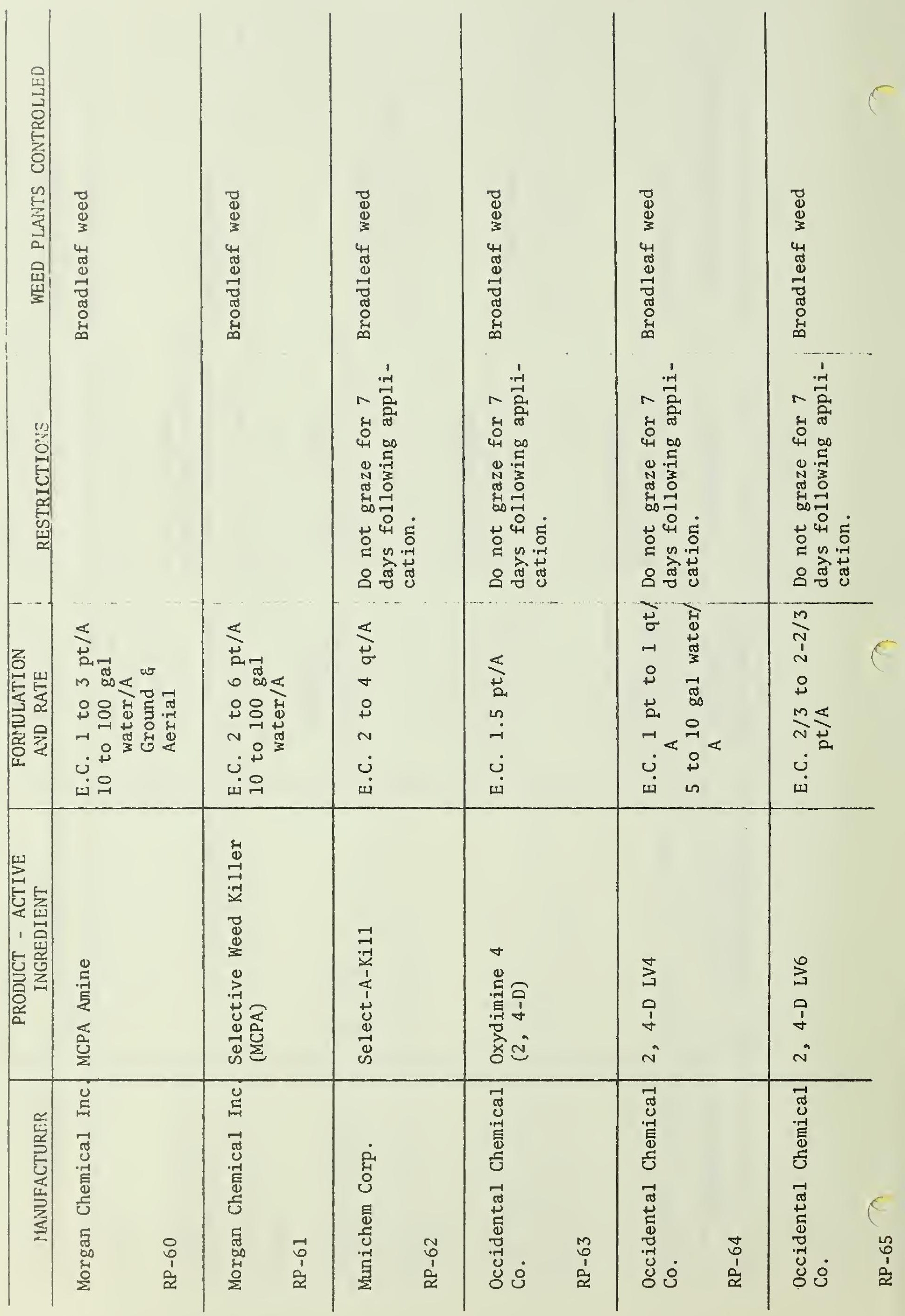




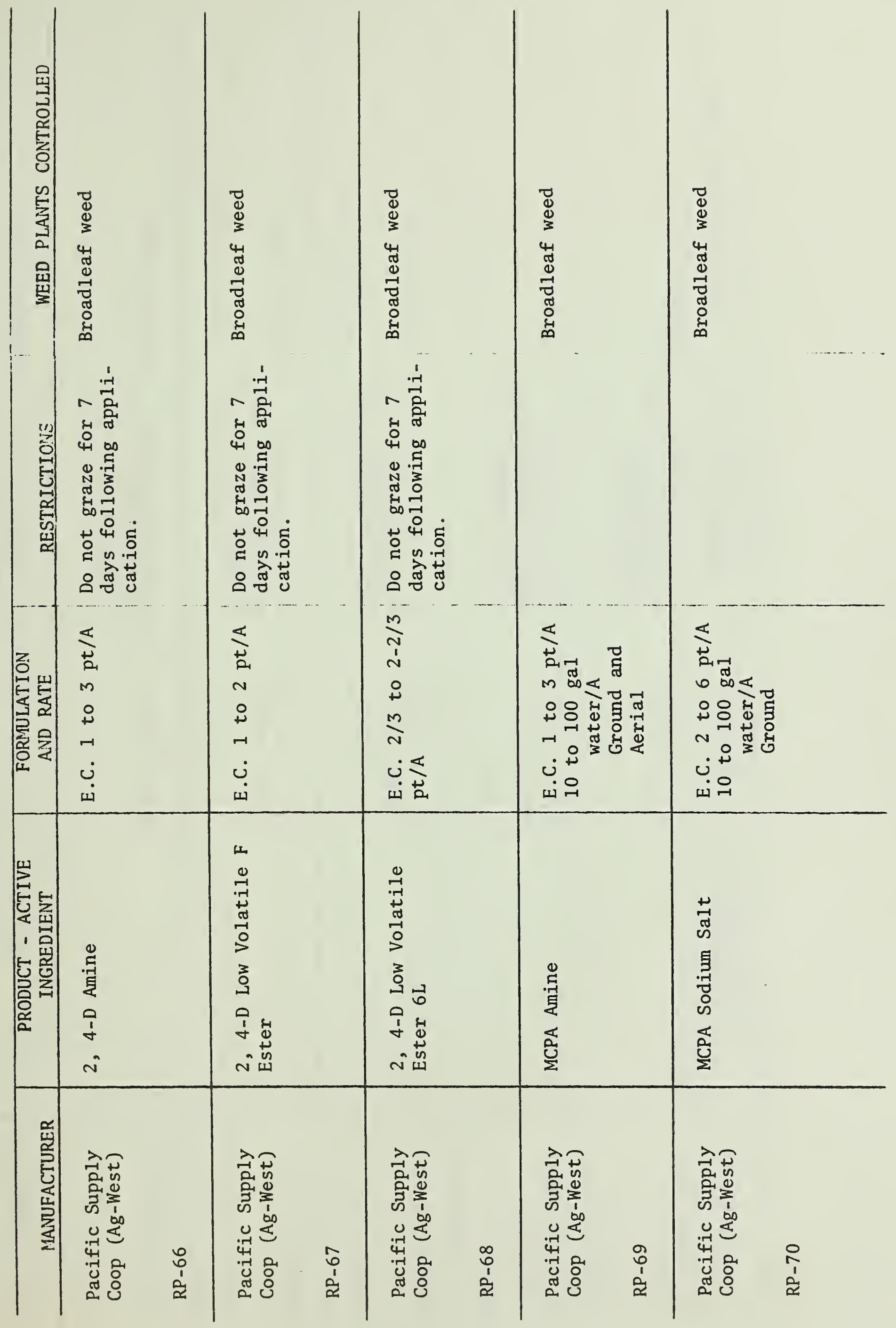




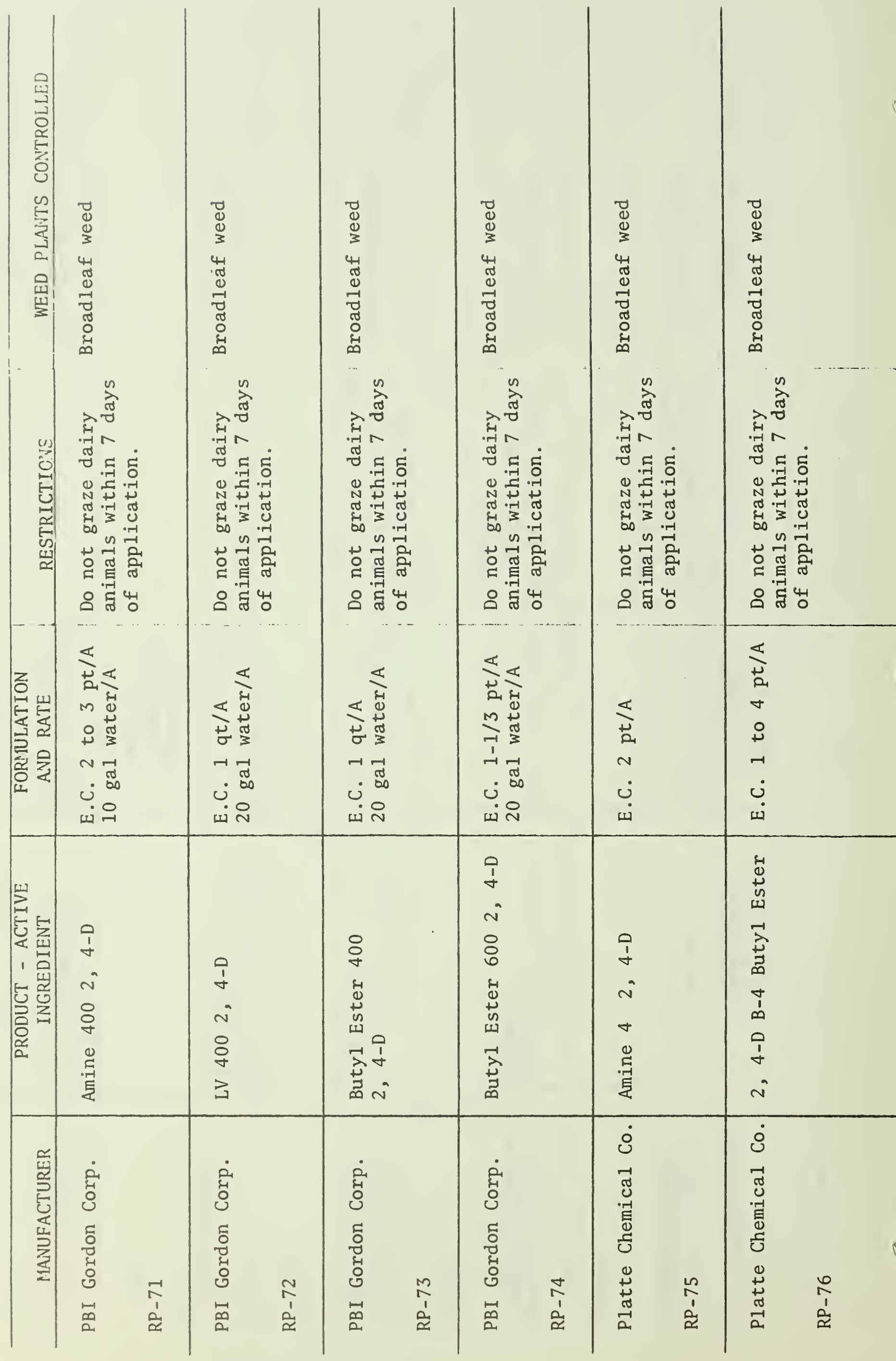




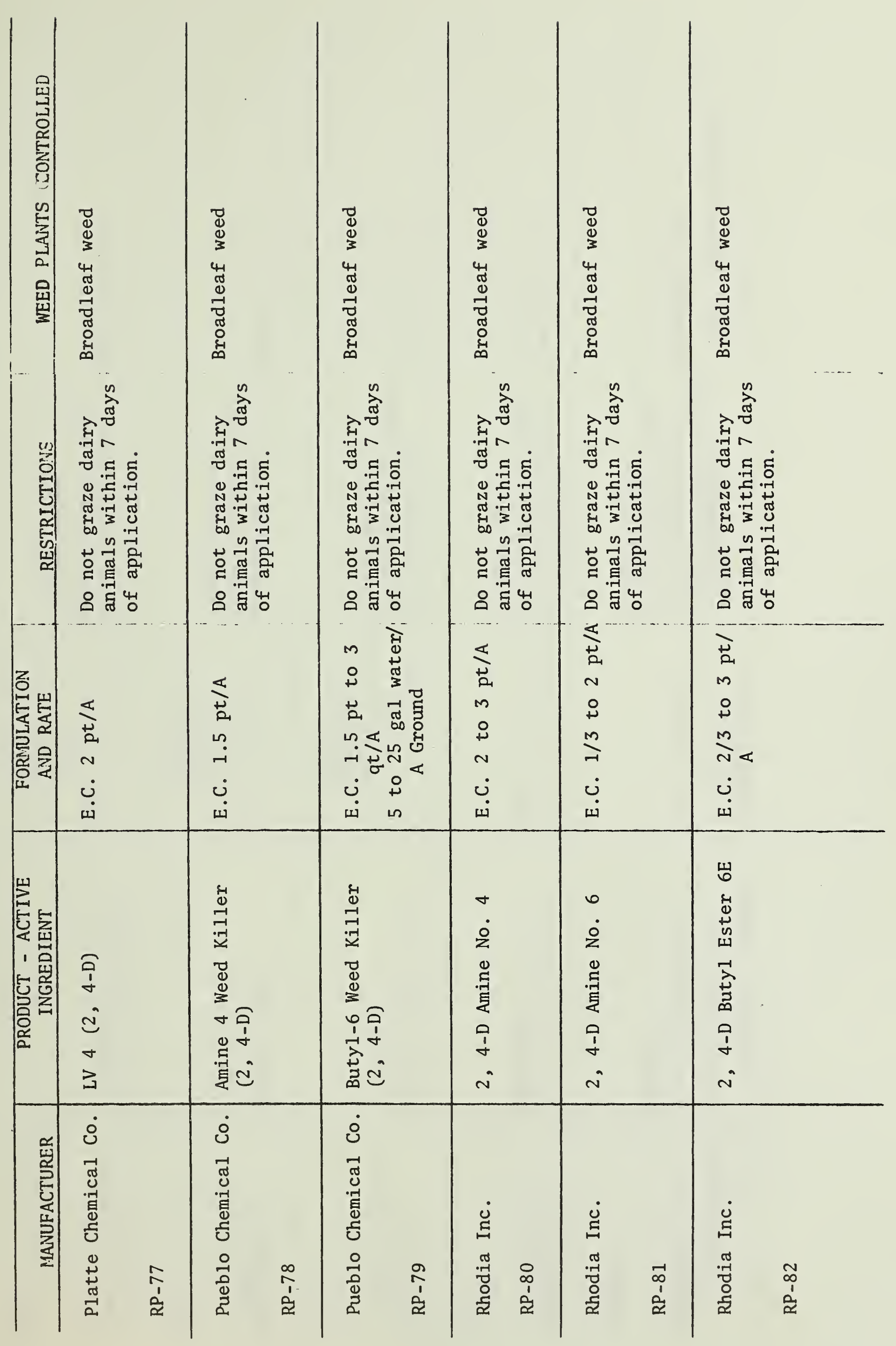




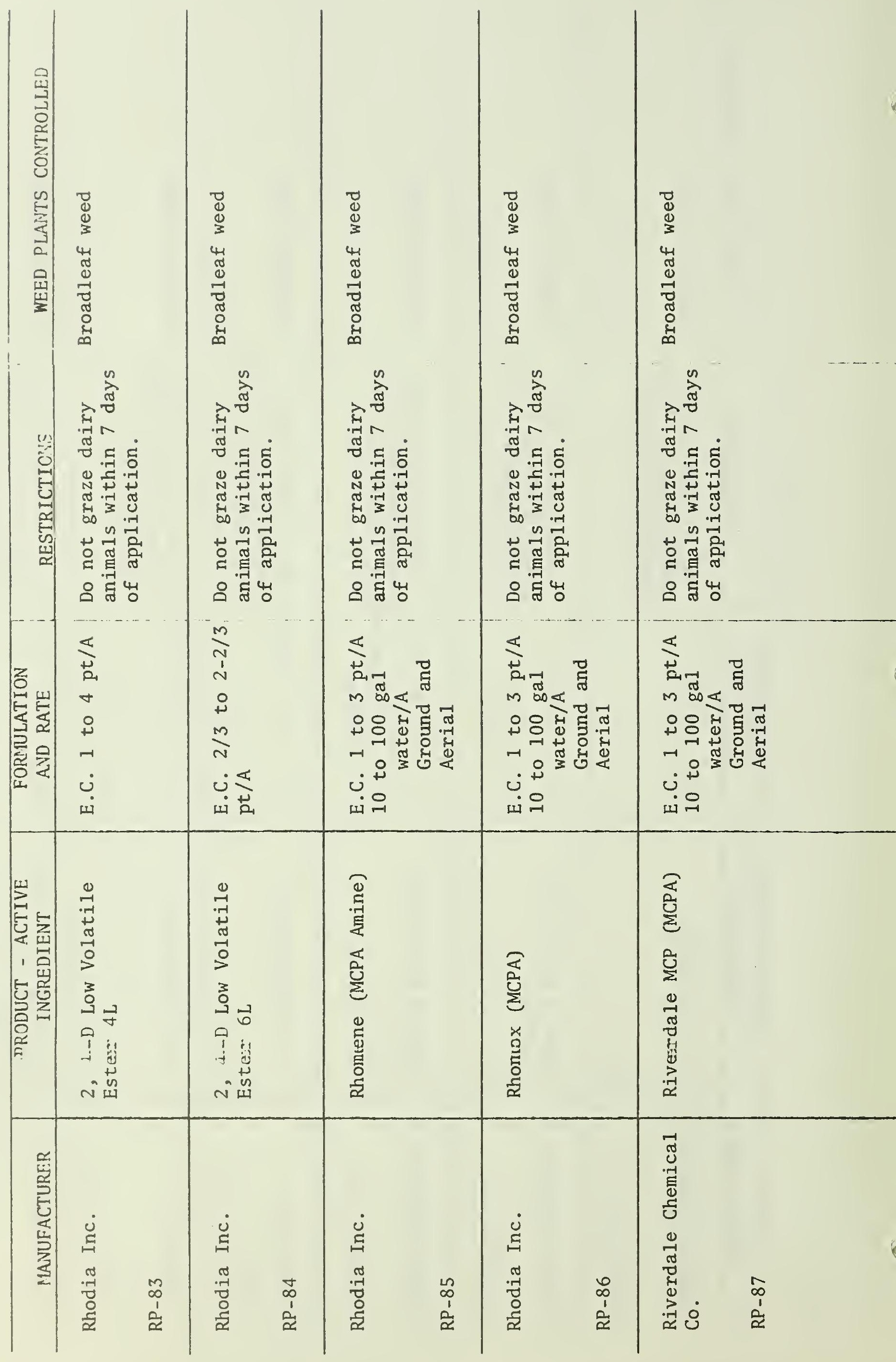




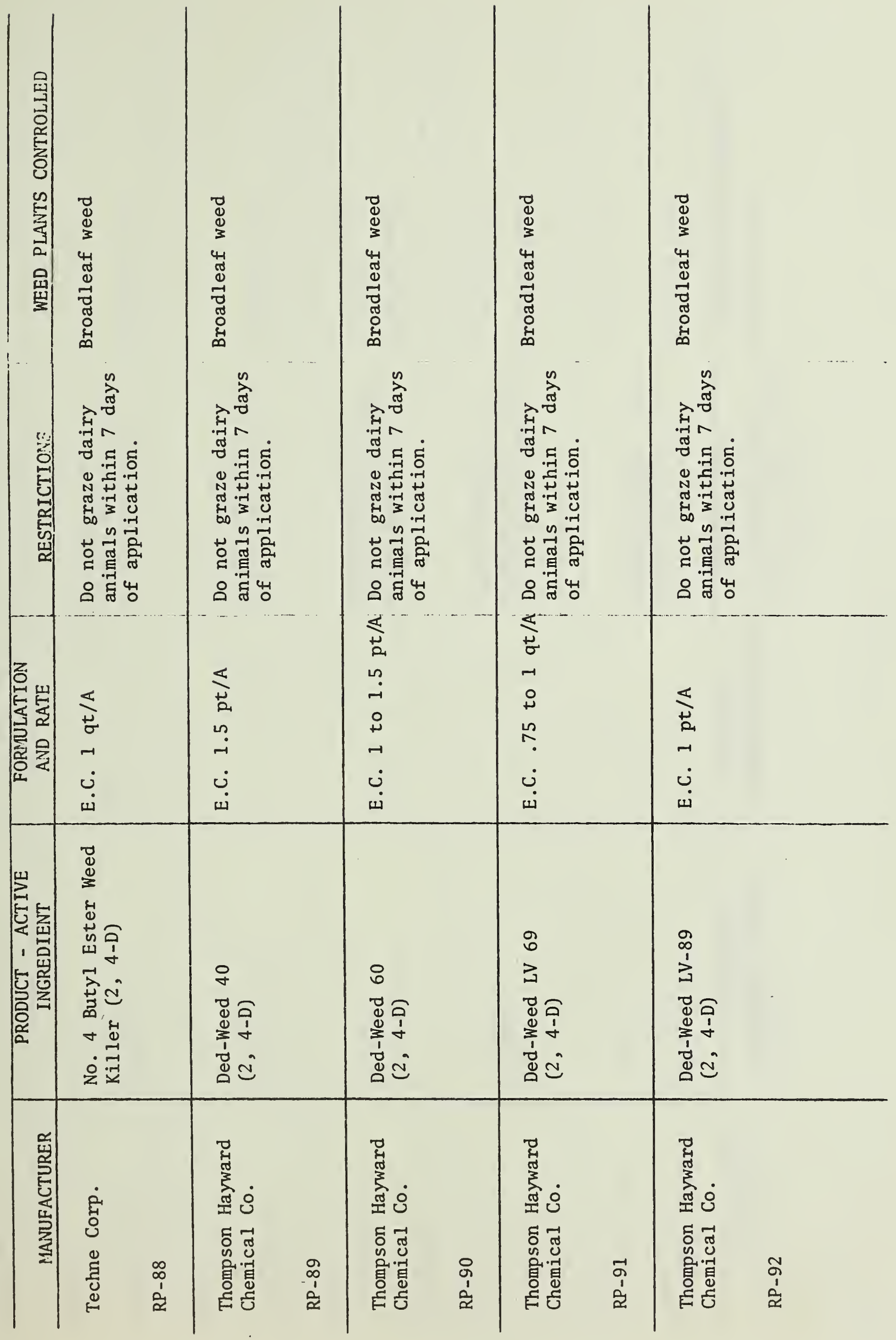




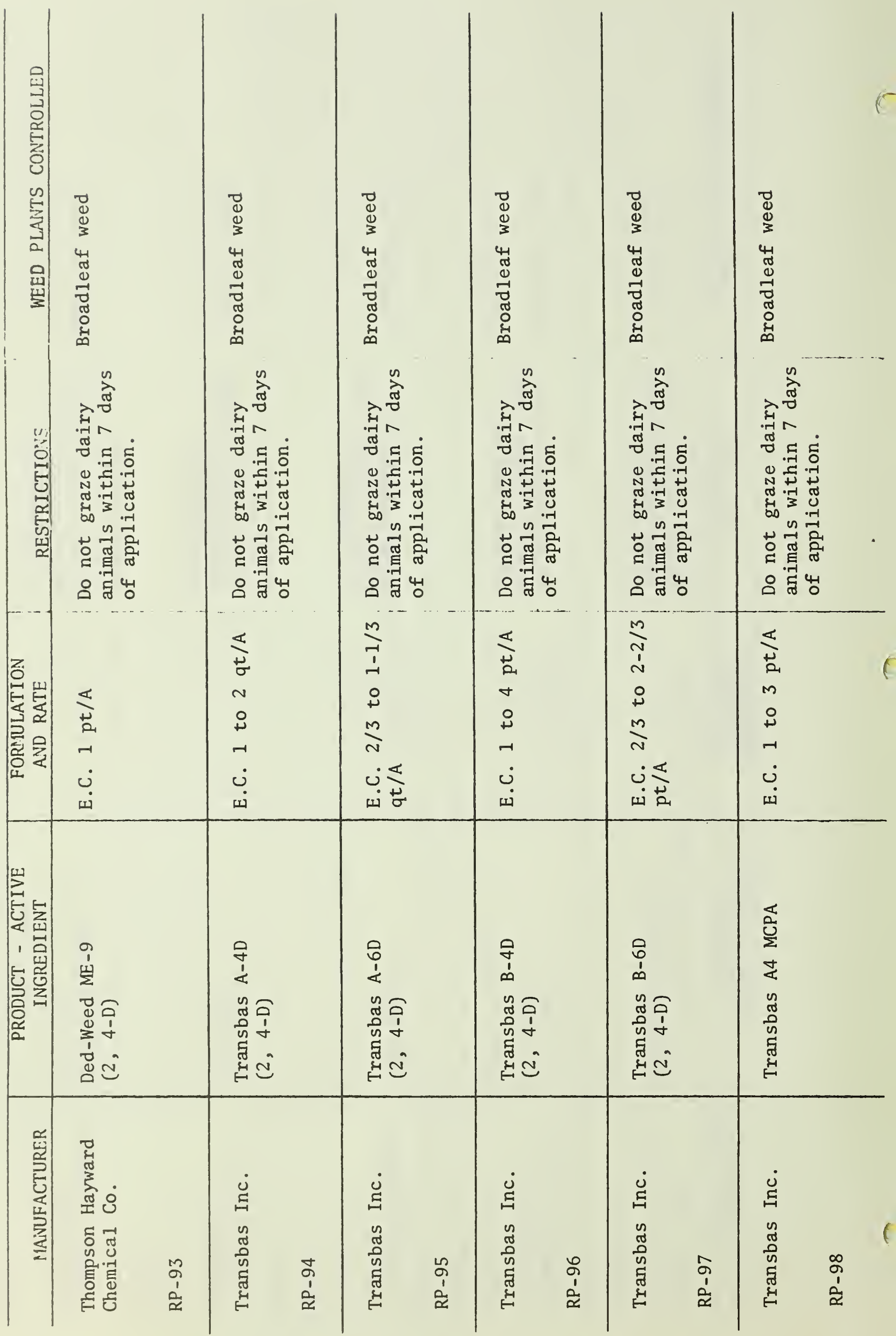




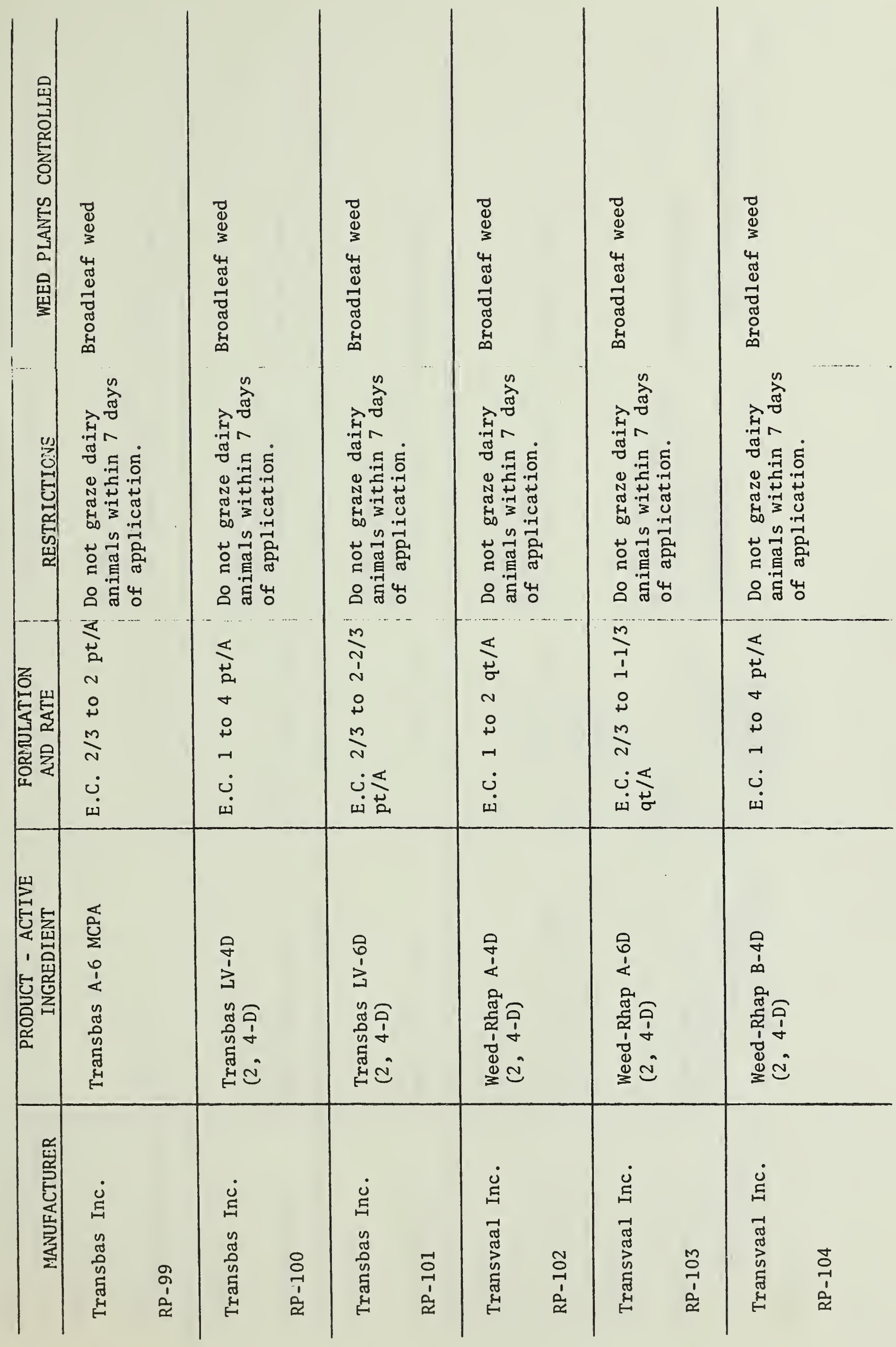




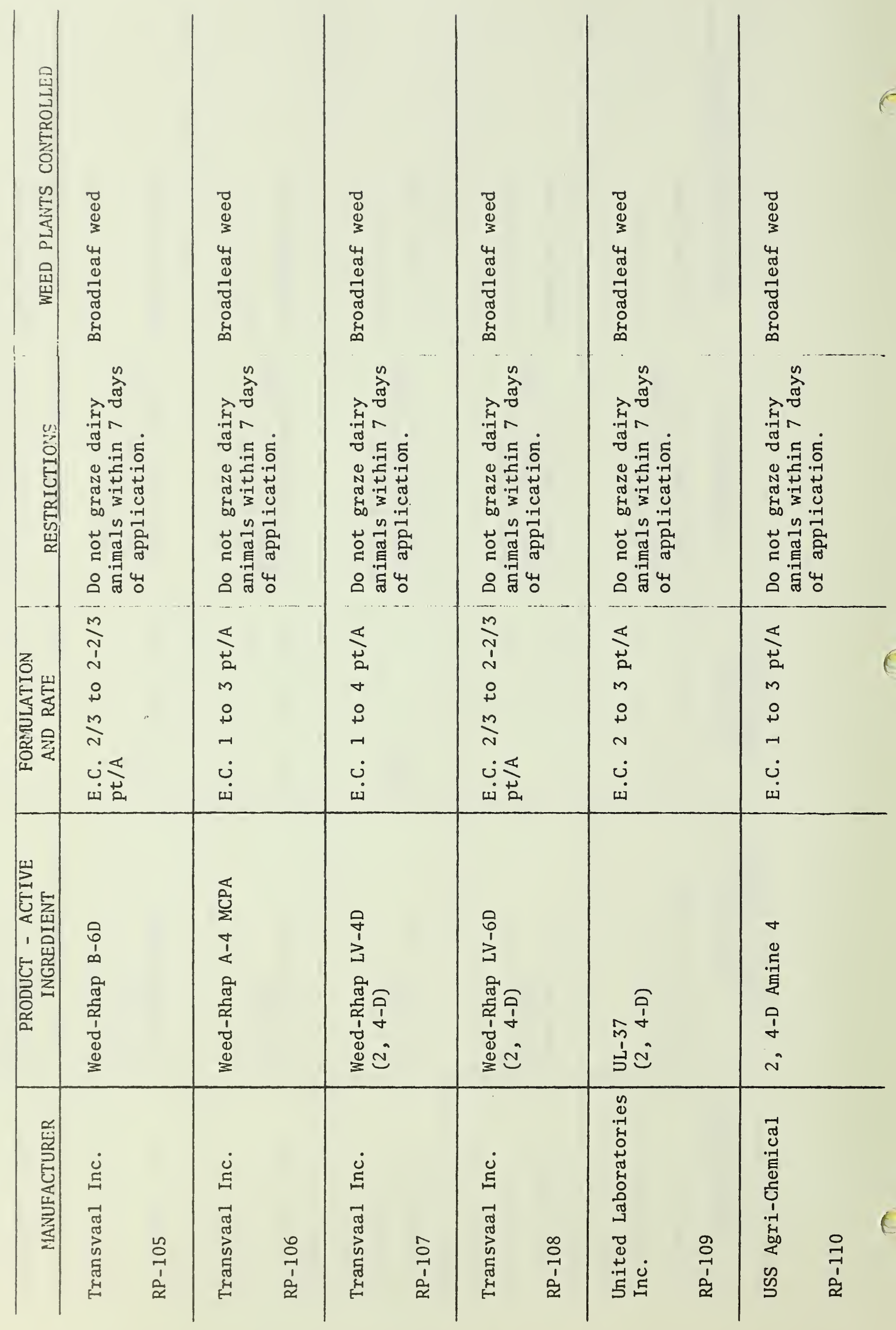




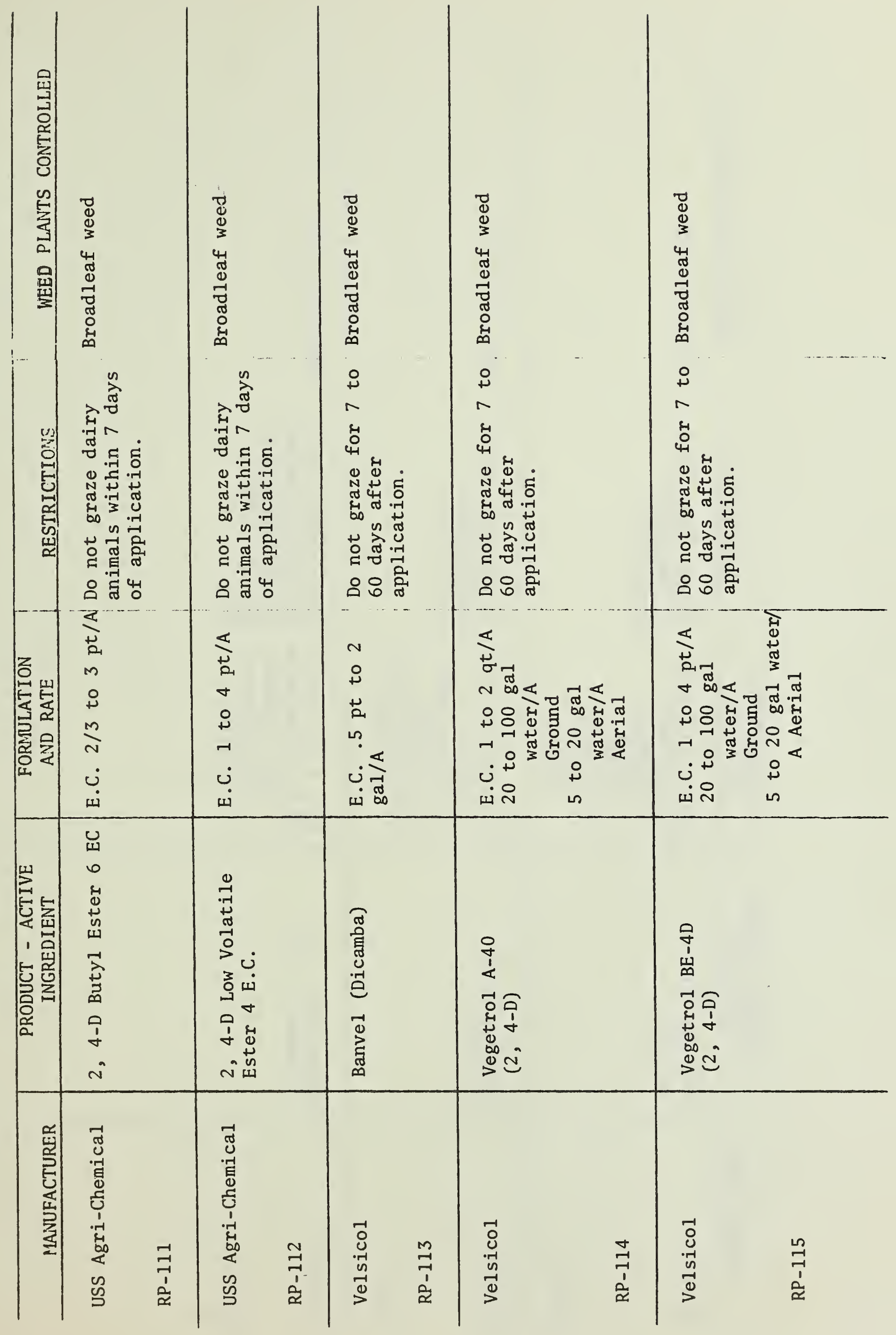




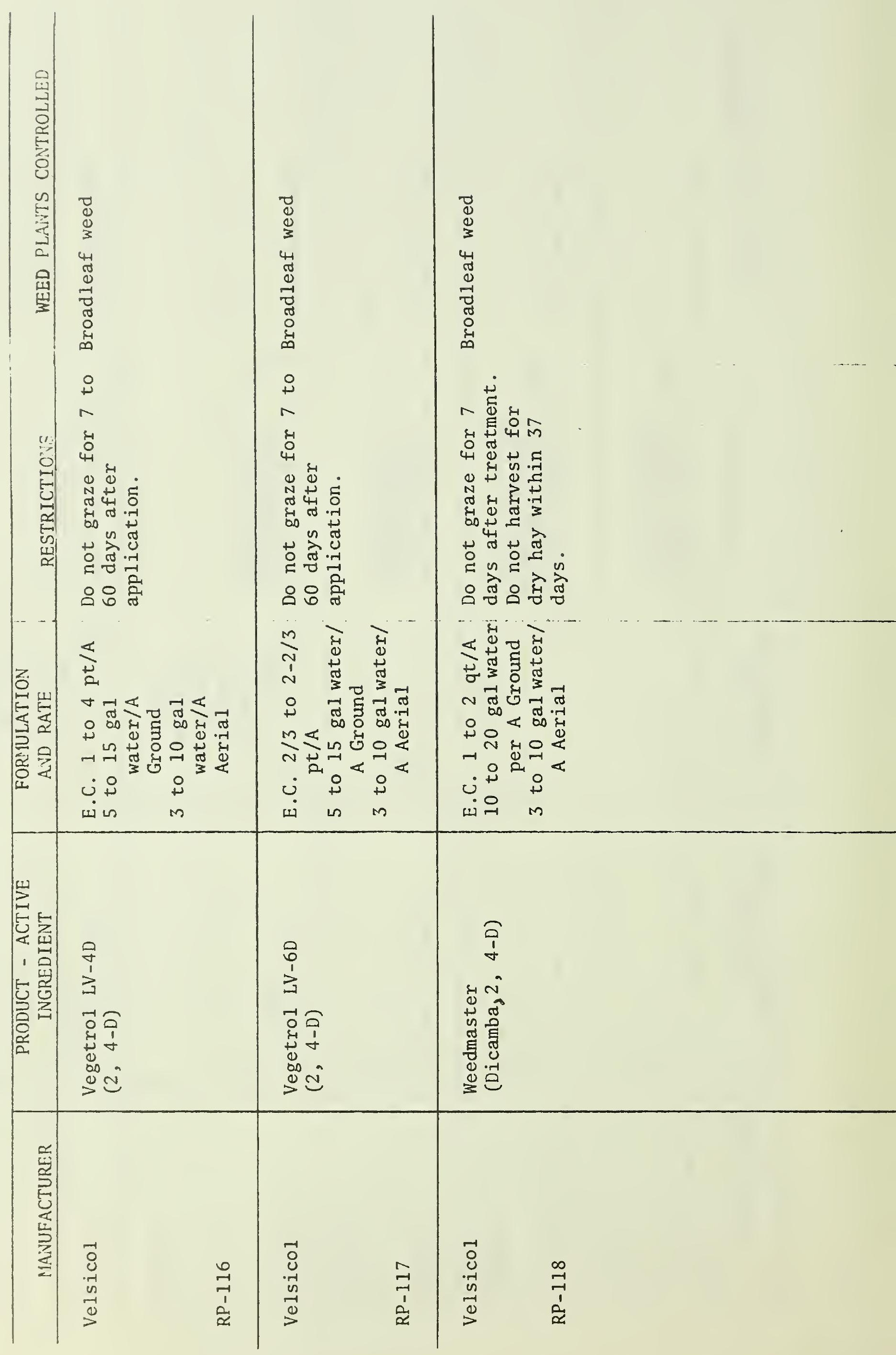



घंल

a a a a

원ำ

r

造

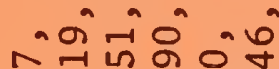

त्न

$\infty 0 \hat{0}$

ปे

नヘ

N

검 $\infty \sigma^{\infty}$

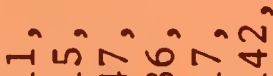

न

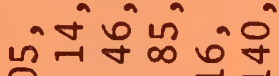

$\rightarrow$ a a न्न

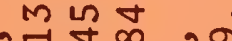

\& $m$

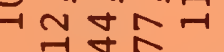

a a

a

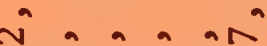

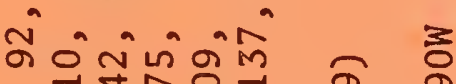

a क वे

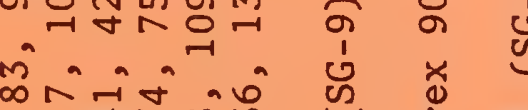

, $\forall+\infty$

$\infty 60 \hat{m}$

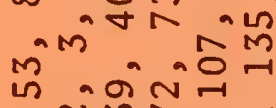

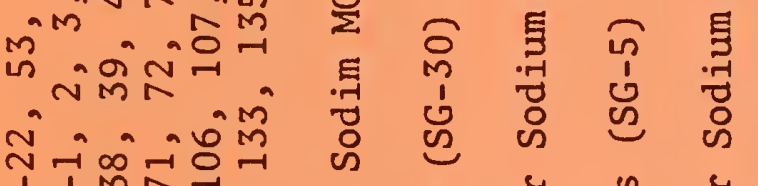

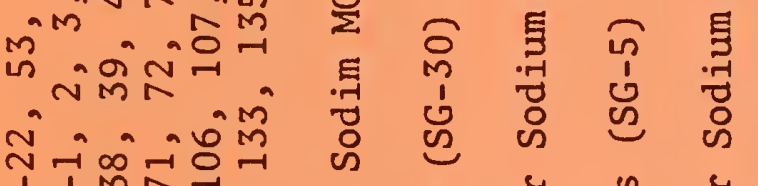

商

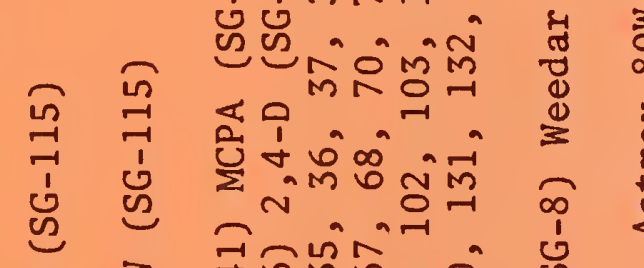

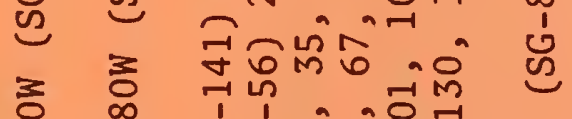

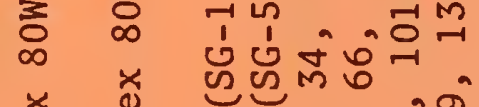

শ đ

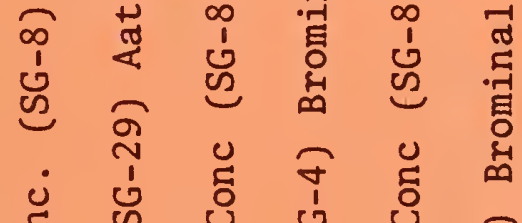

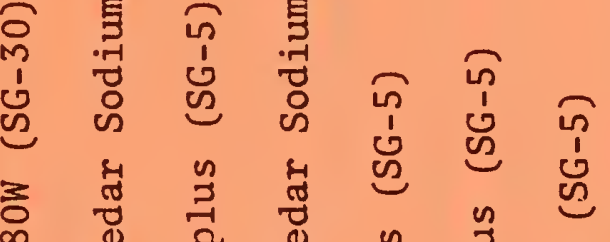

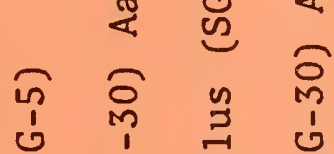

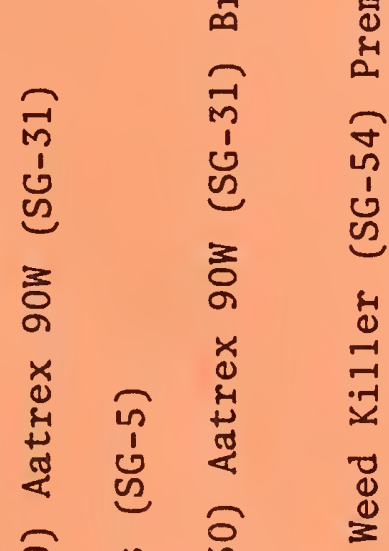

$\geq 0$

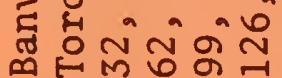

ن.

$\ddot{g}$

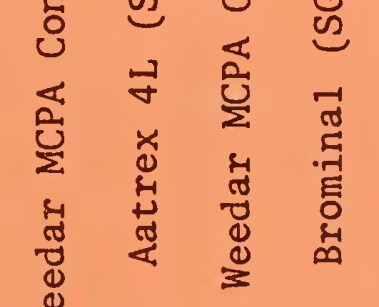

造

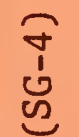

f

占

(1)

क ज्यें

न 3 年

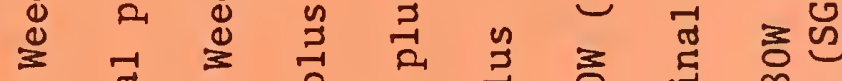

a

की

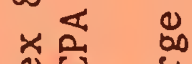

出

目 空

통

势

于 要目

啘

ลํำ

สุธ

居 总

氜

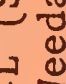

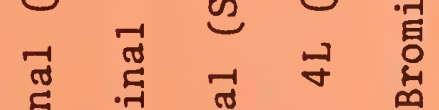

桴

×ळ

인

䨪

苜

帒

i. 


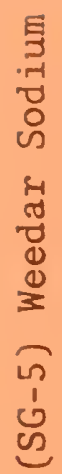

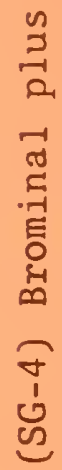

离

क

क्ञ

2
$\infty$
1
ల్

胥

署

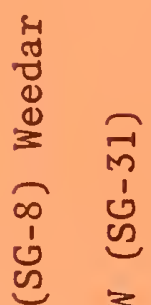

สิ

峁 $\sigma$ क

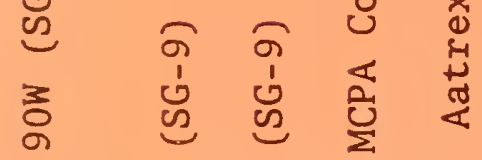

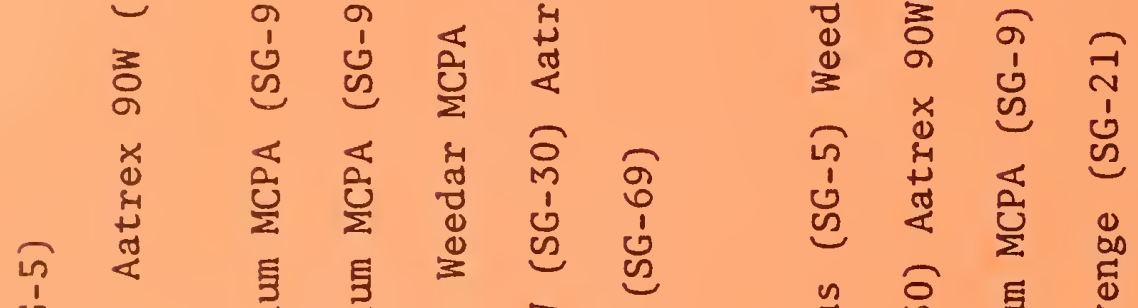

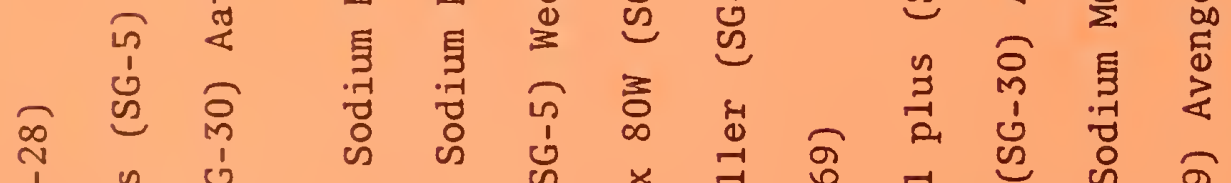

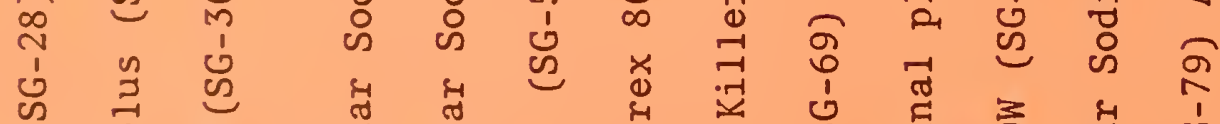

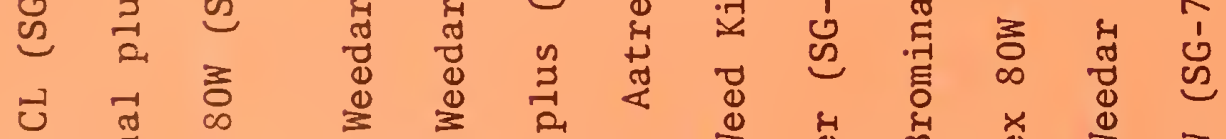

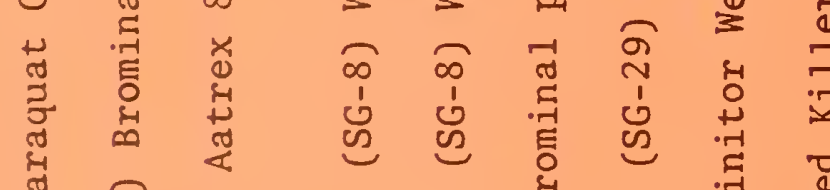

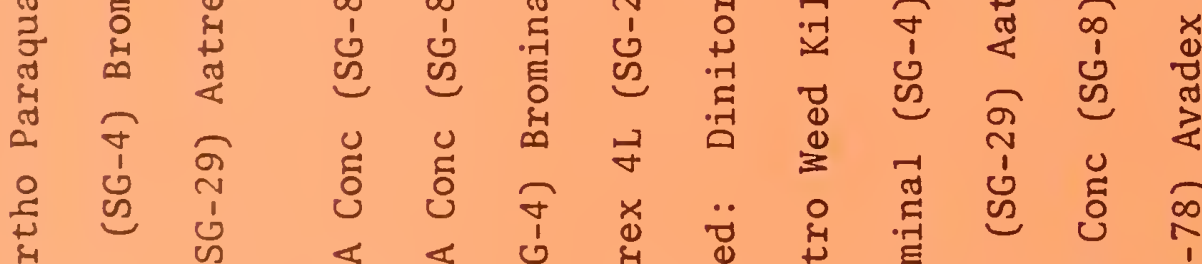

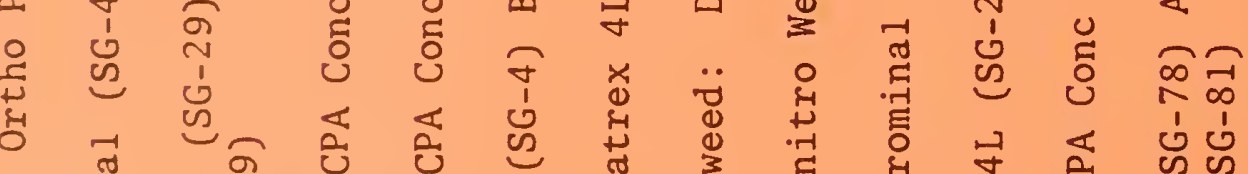

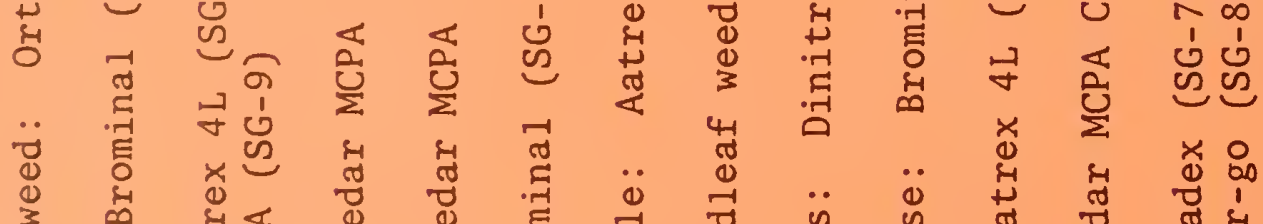

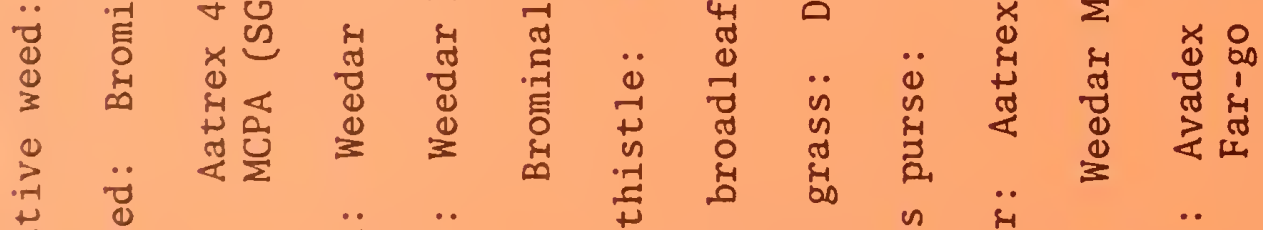

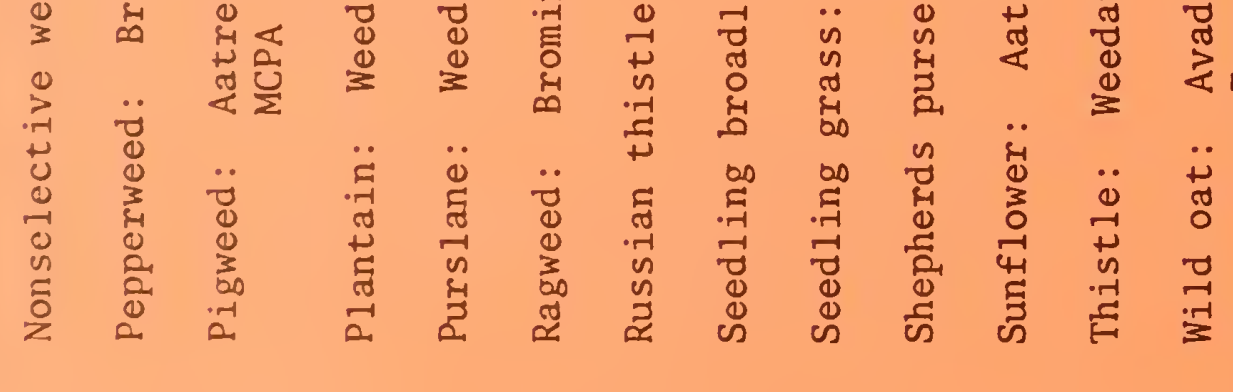




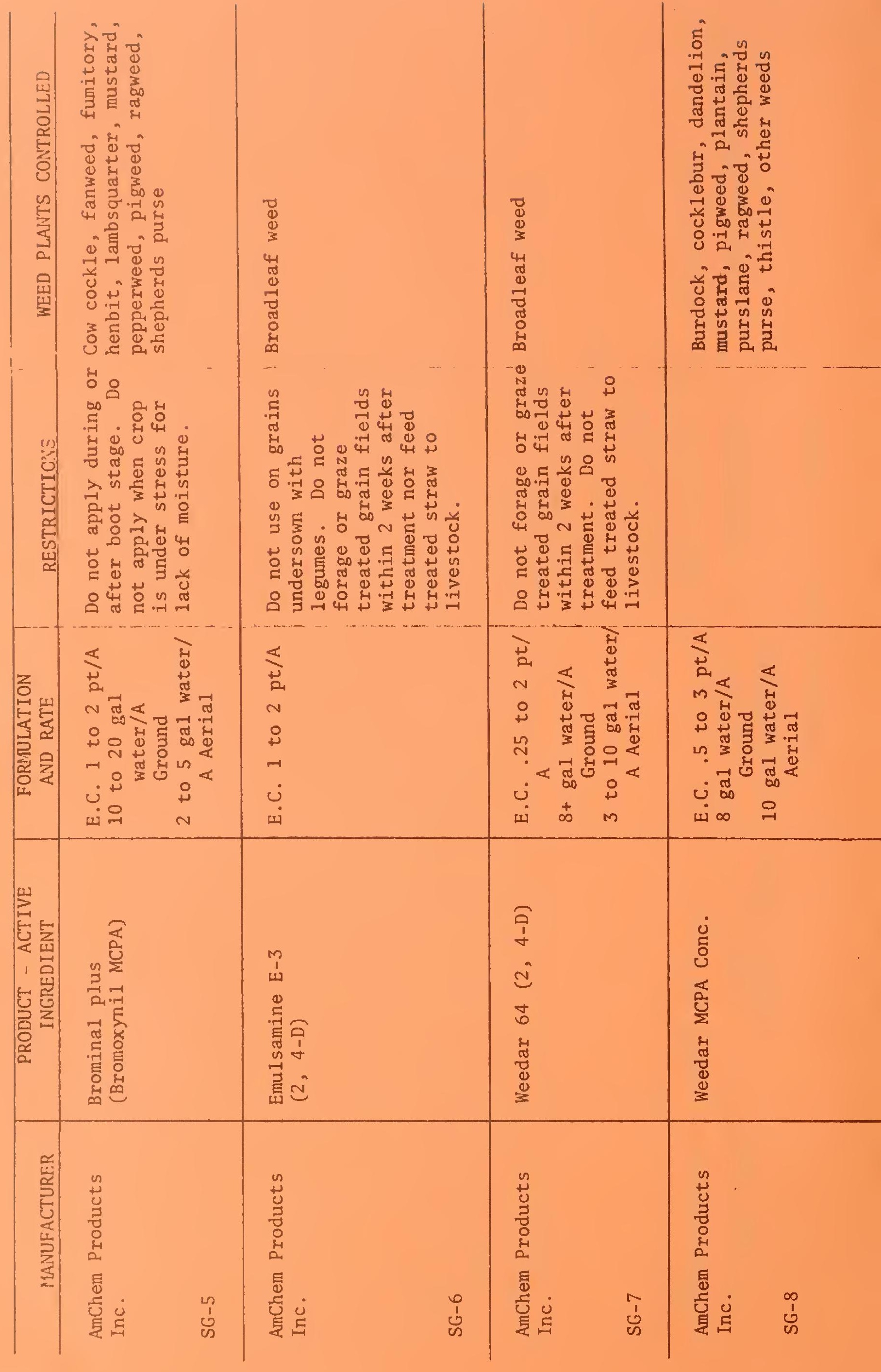




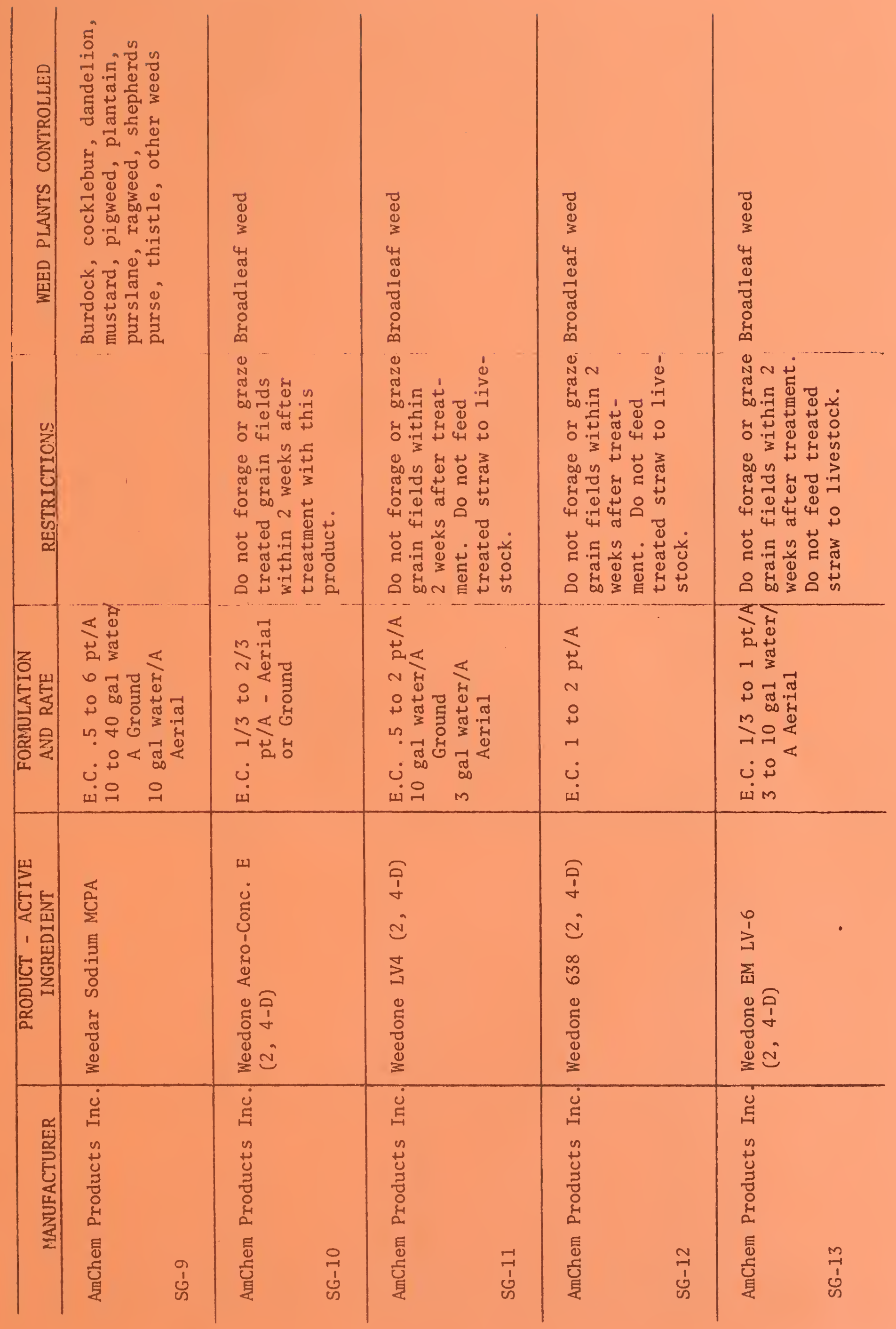




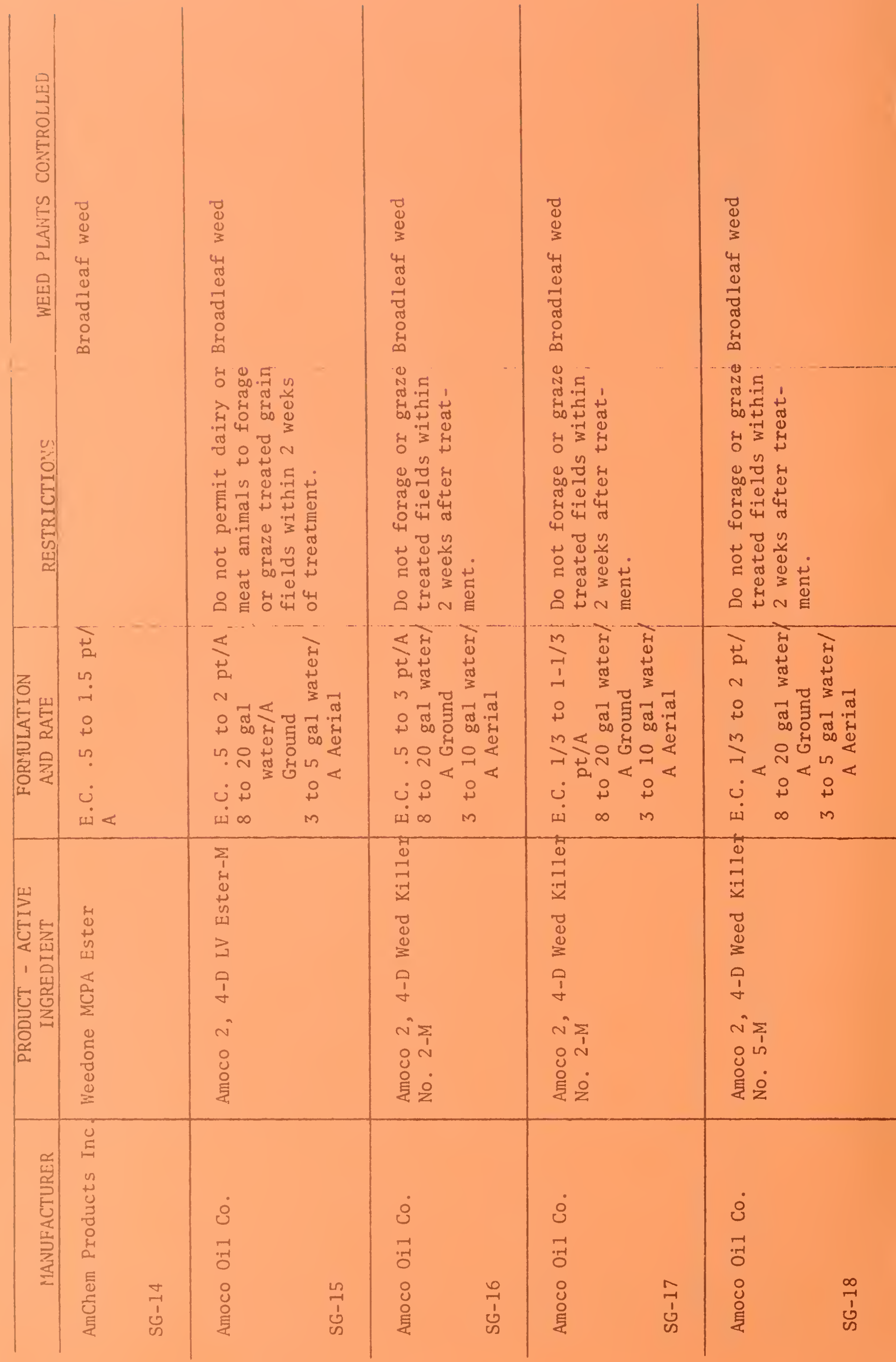




\begin{tabular}{|c|c|c|c|c|c|}
\hline 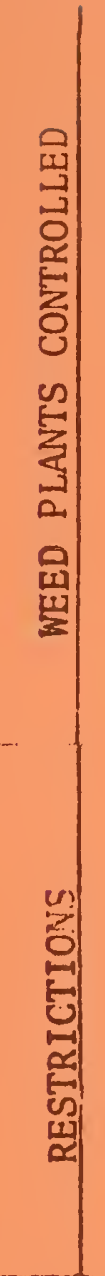 & 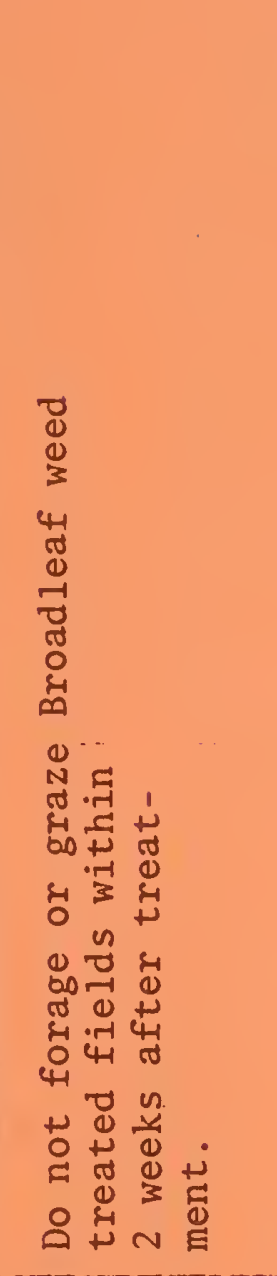 & 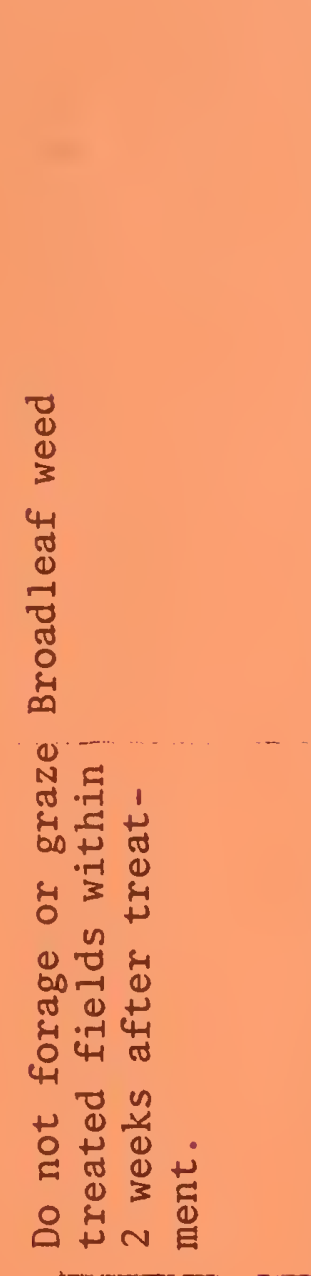 & 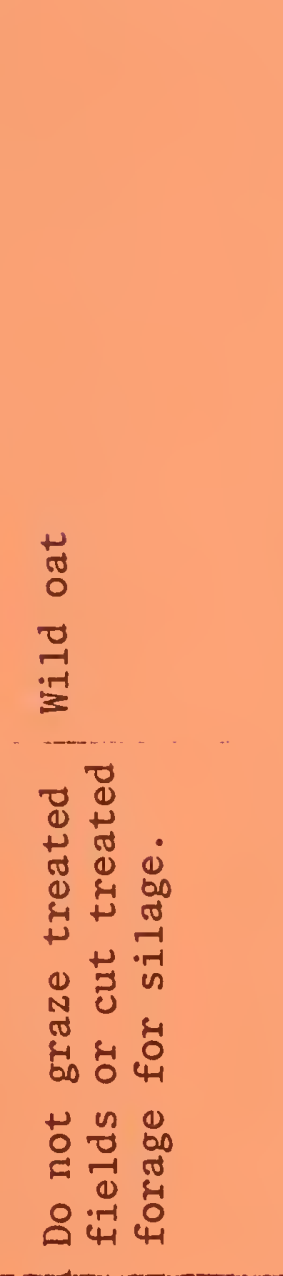 & 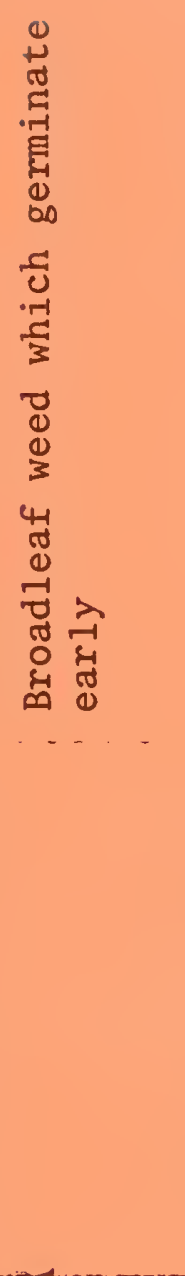 & 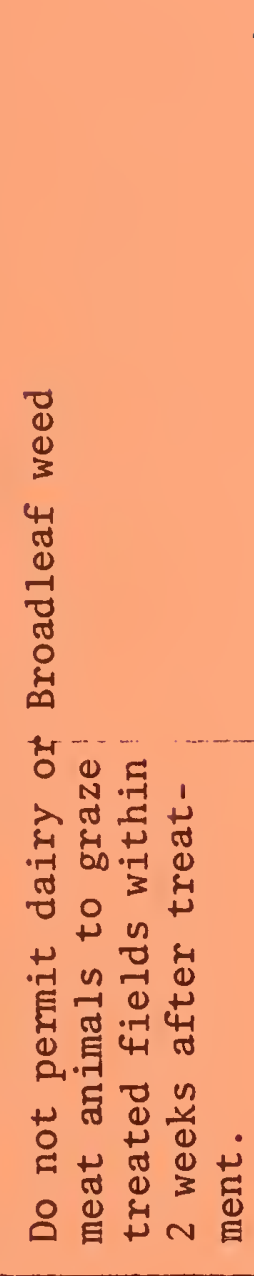 \\
\hline & 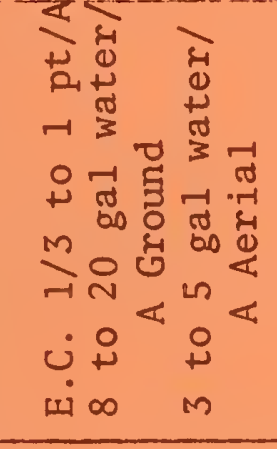 & 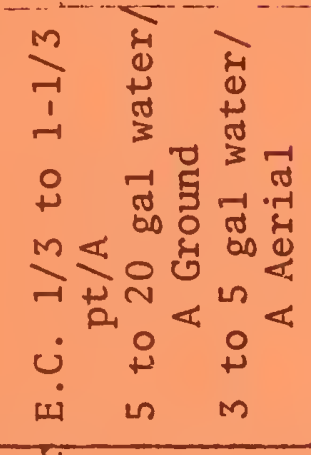 & 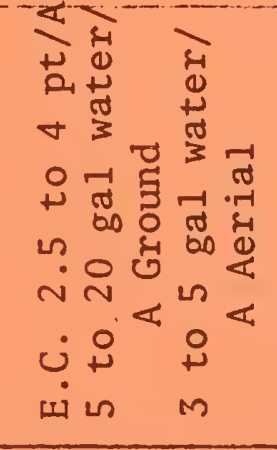 & 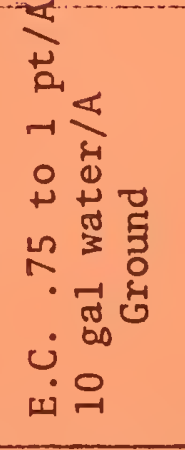 & 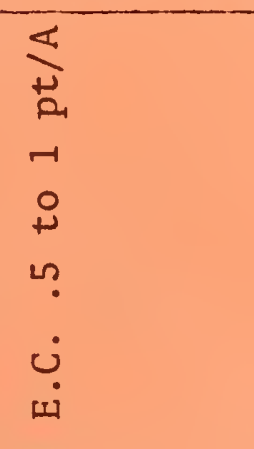 \\
\hline 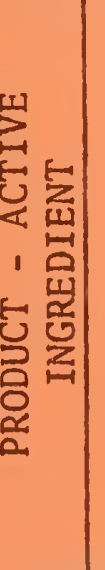 & 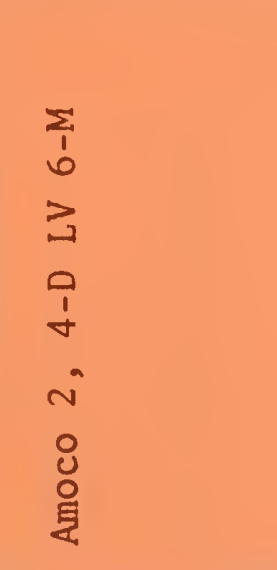 & 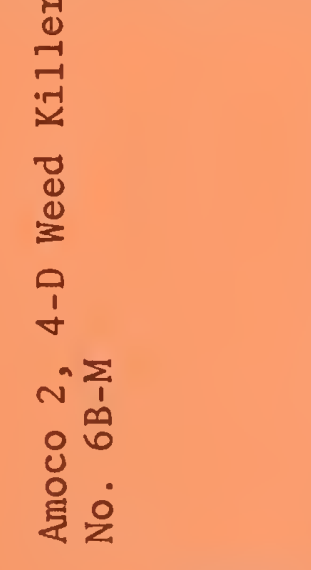 & 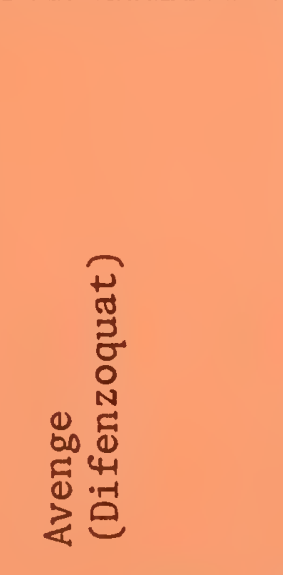 & 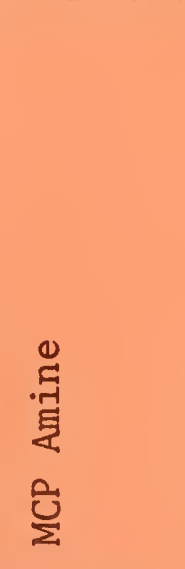 & 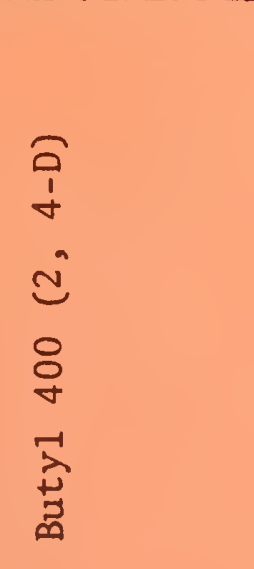 \\
\hline 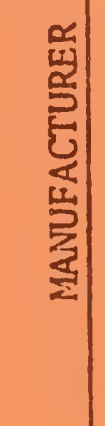 & 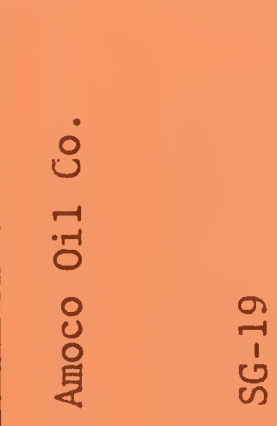 & $\begin{array}{l}\dot{0} \\
\dot{0} \\
\dot{0} \\
\dot{0} \\
0 \\
\dot{0}\end{array}$ & 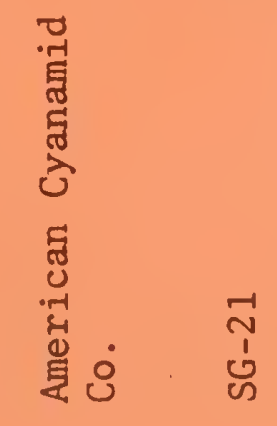 & $\begin{array}{l}\stackrel{㐅}{凶} \\
\stackrel{\Xi}{0}\end{array}$ & 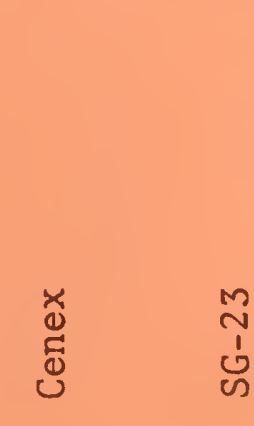 \\
\hline
\end{tabular}




\begin{tabular}{|c|c|c|c|c|c|}
\hline 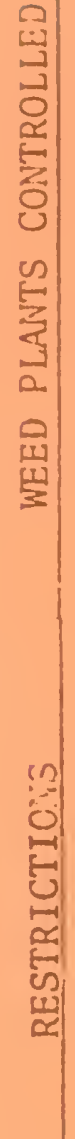 & 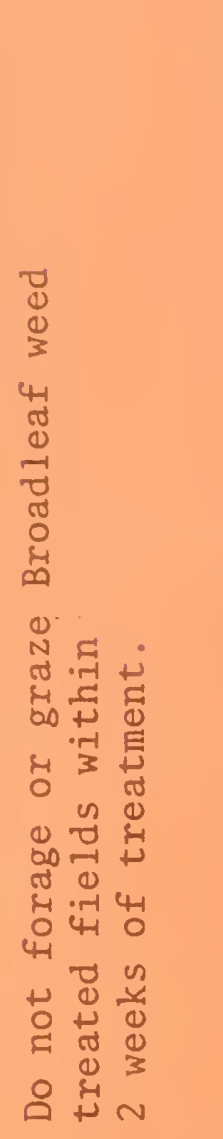 & 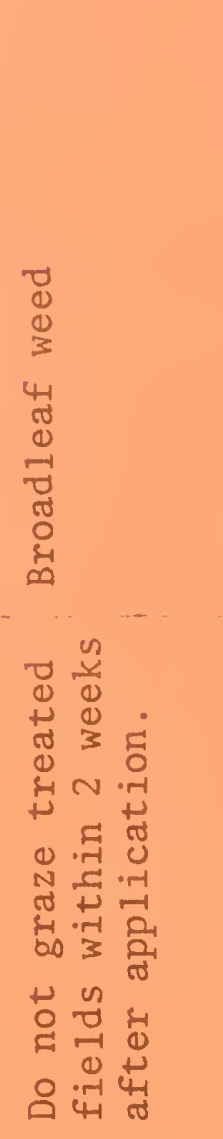 & 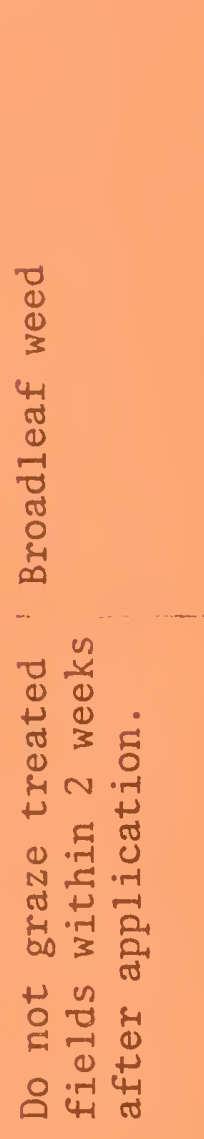 & 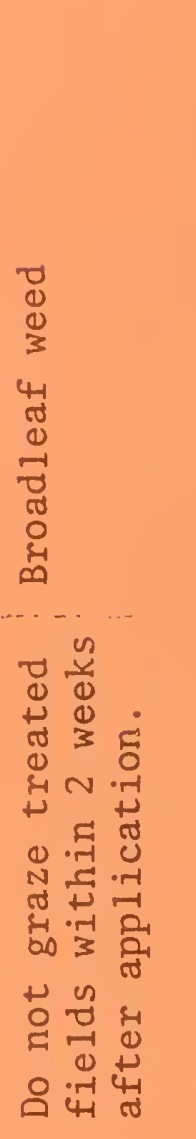 & 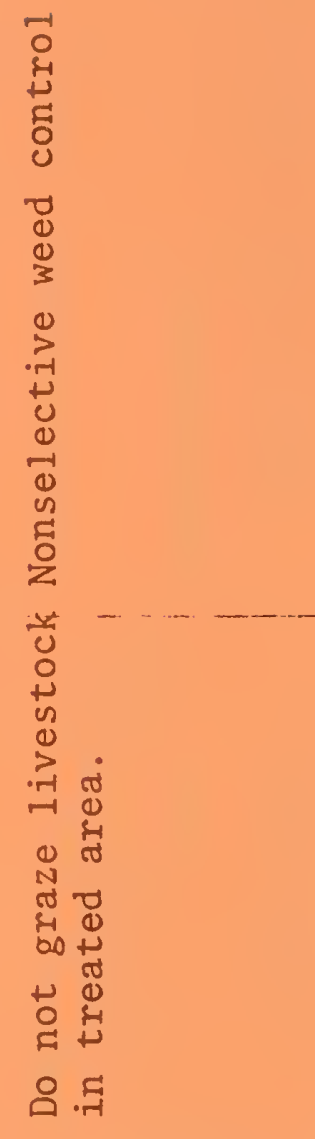 \\
\hline 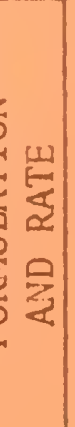 & 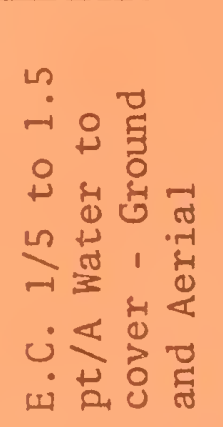 & 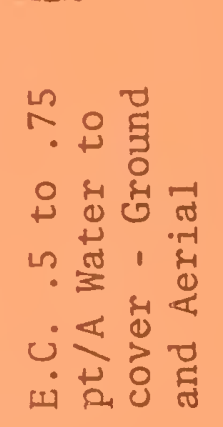 & 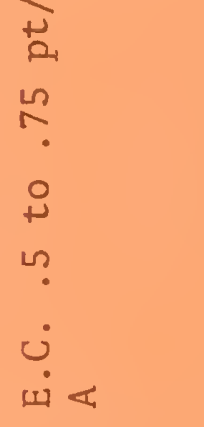 & 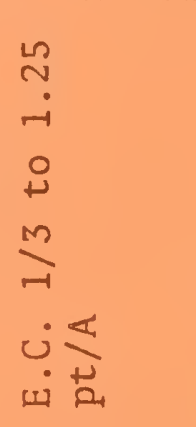 & 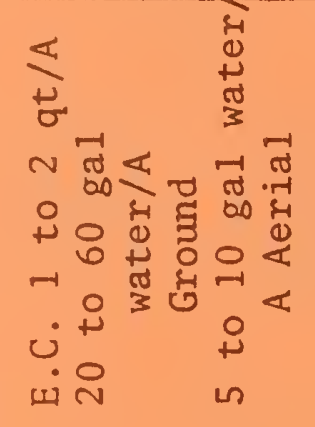 \\
\hline 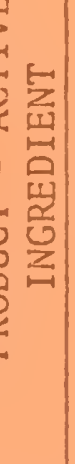 & 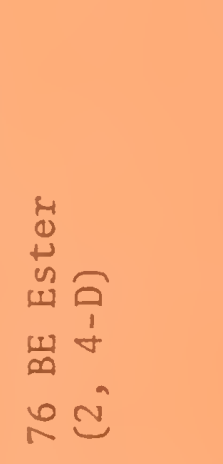 & 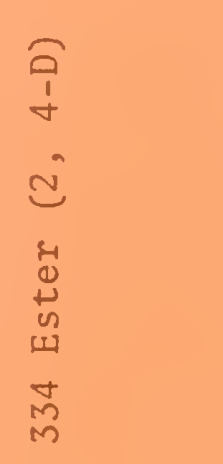 & 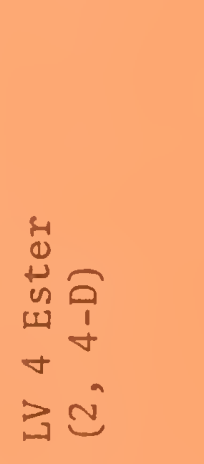 & 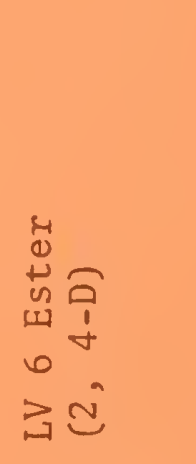 & 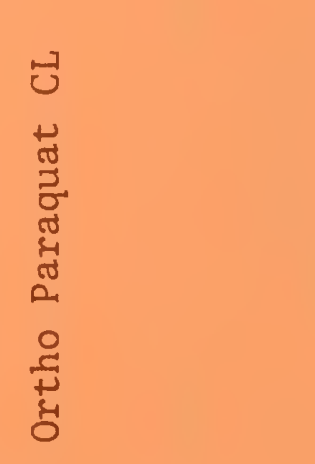 \\
\hline 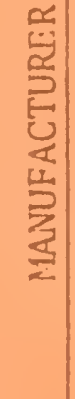 & 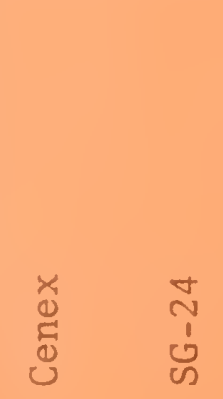 & 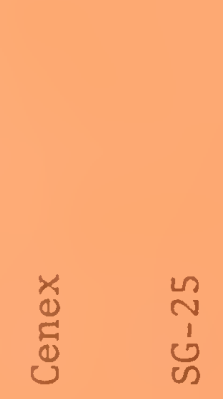 & 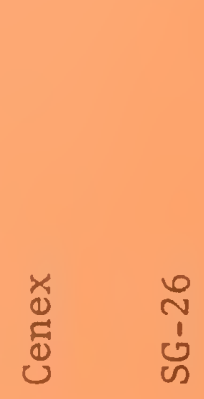 & 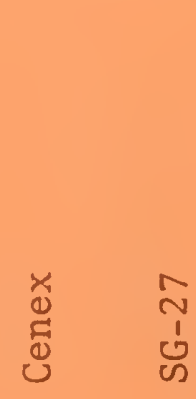 & 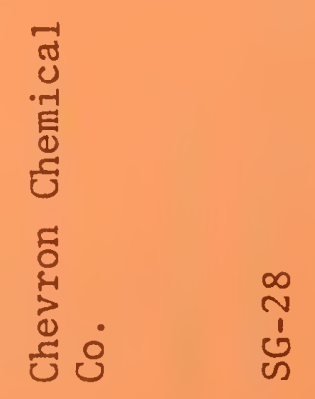 \\
\hline
\end{tabular}




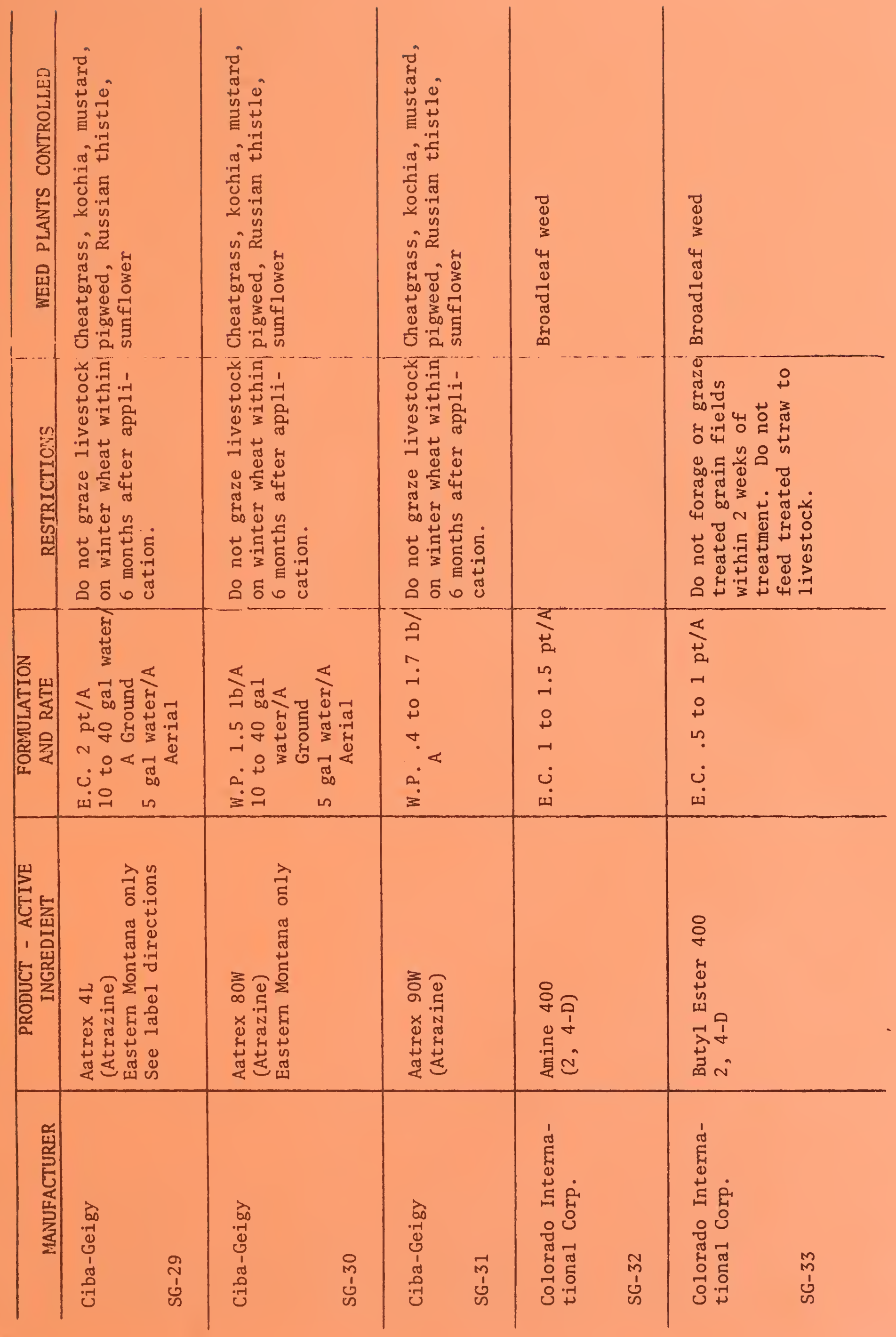




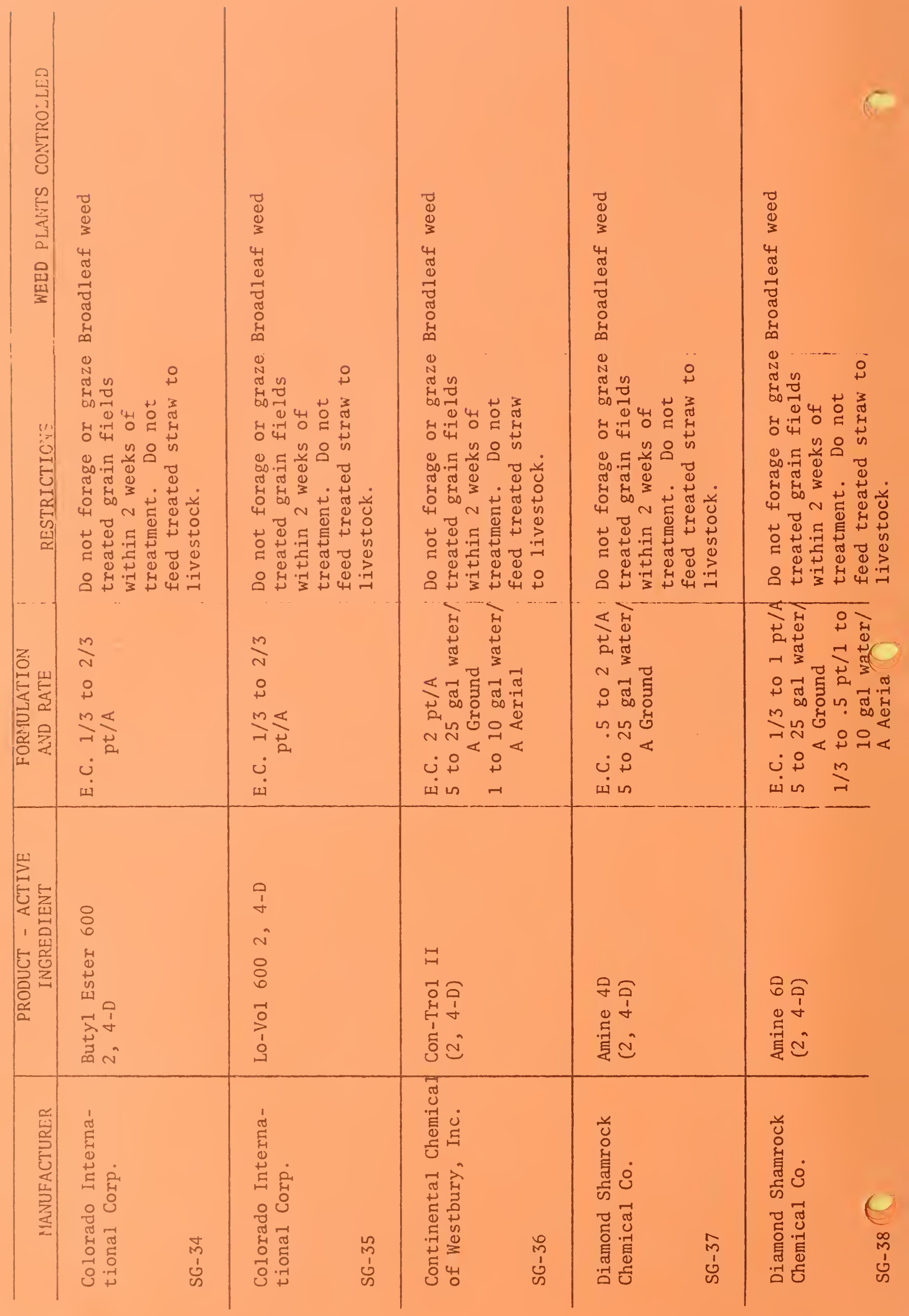




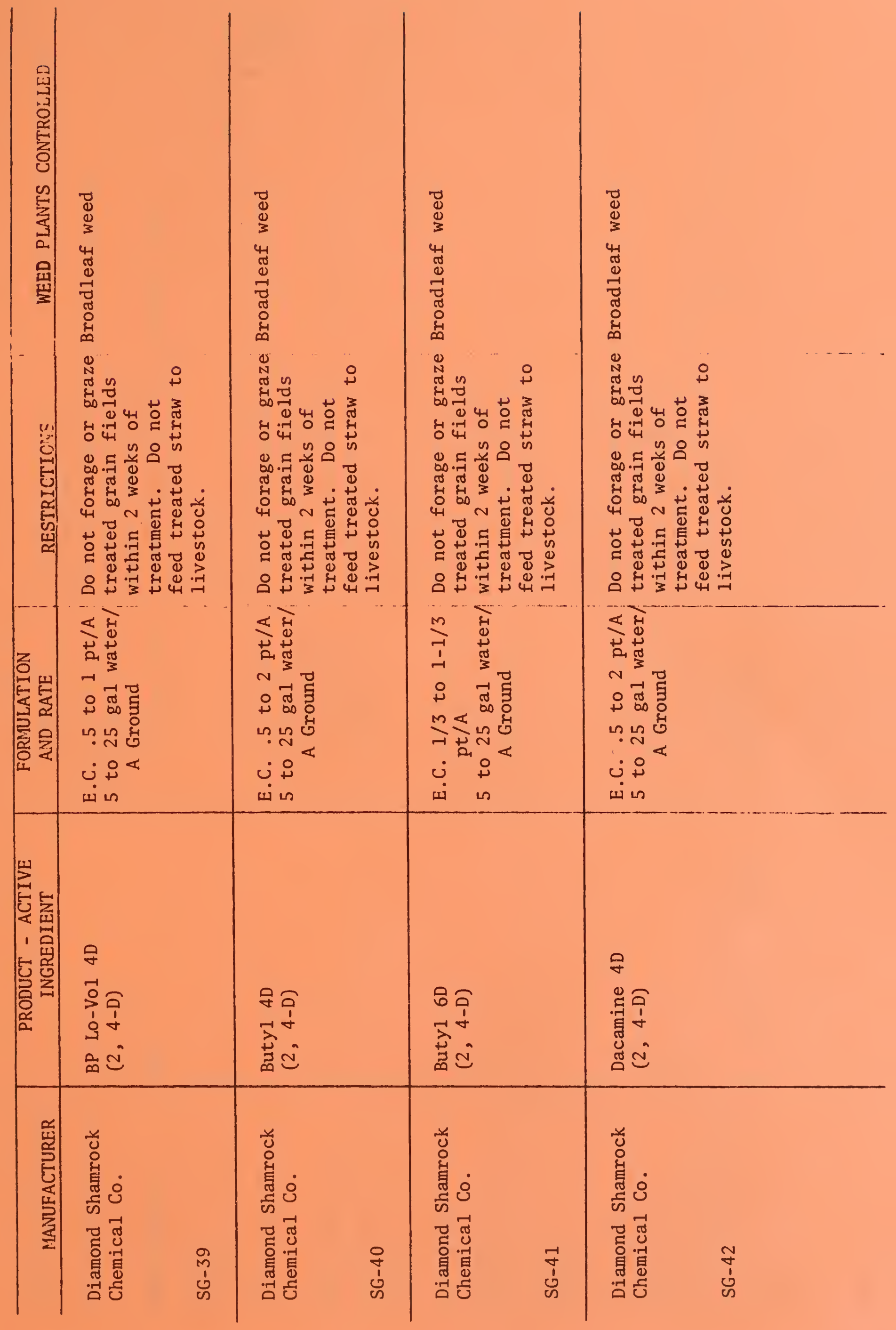




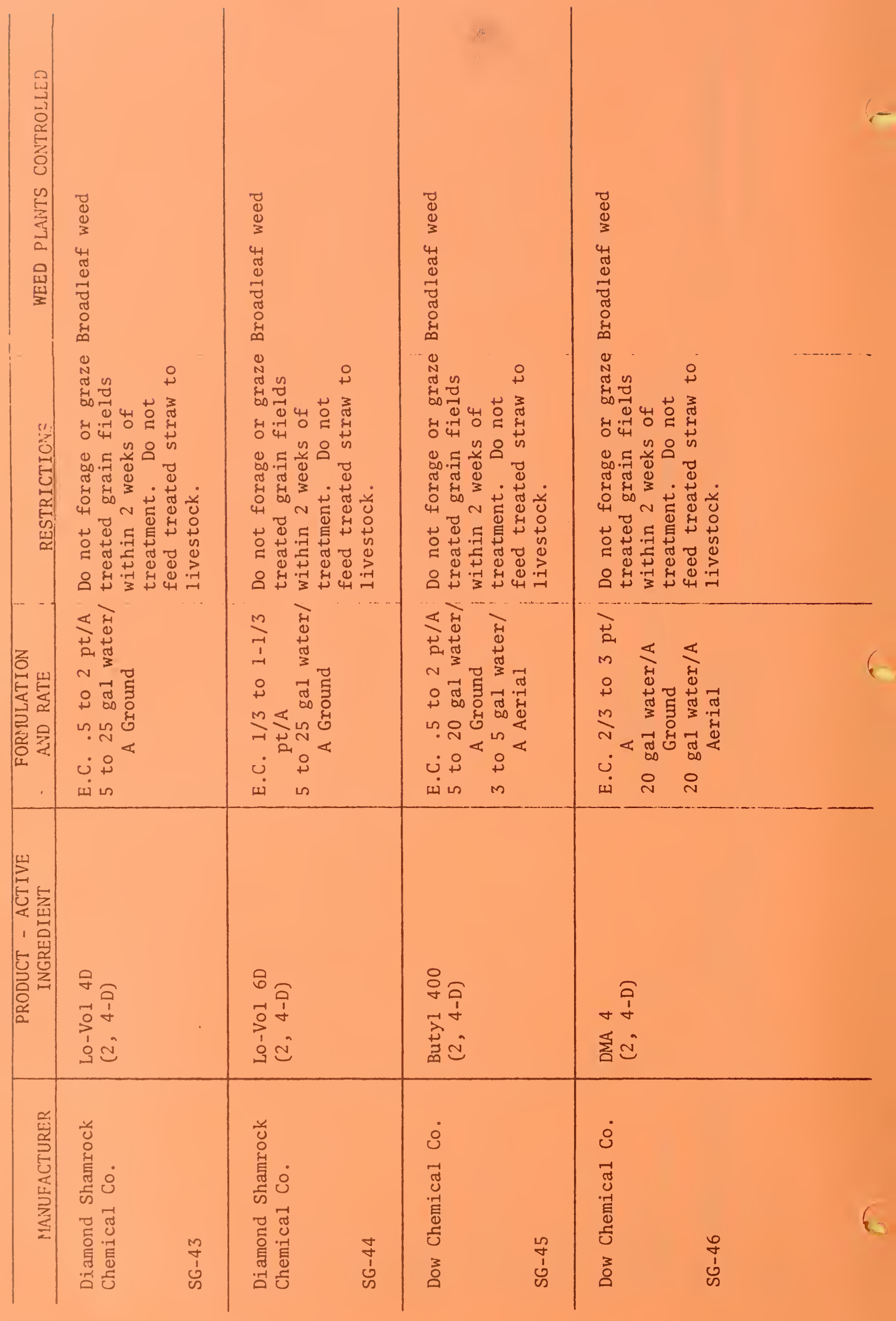




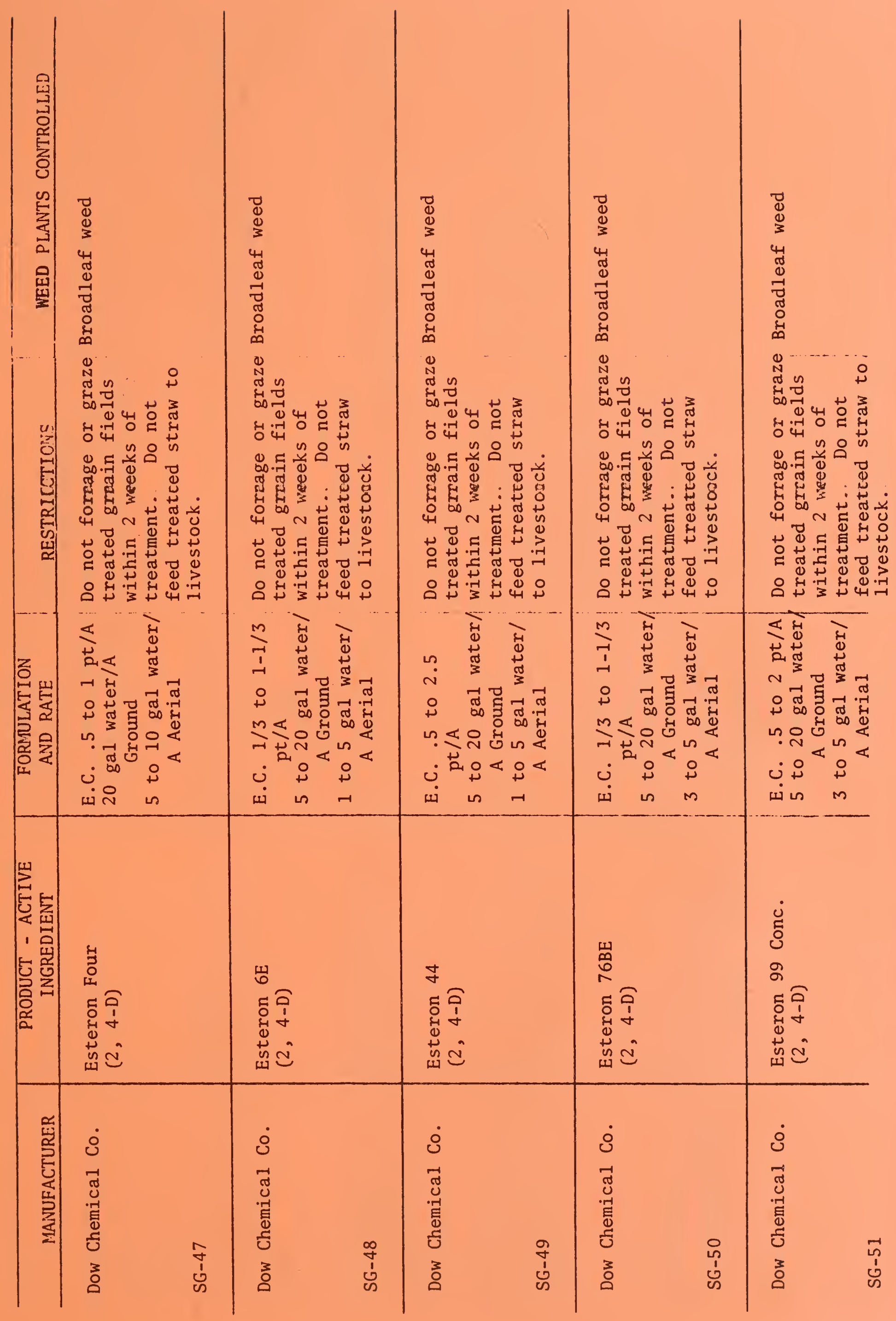




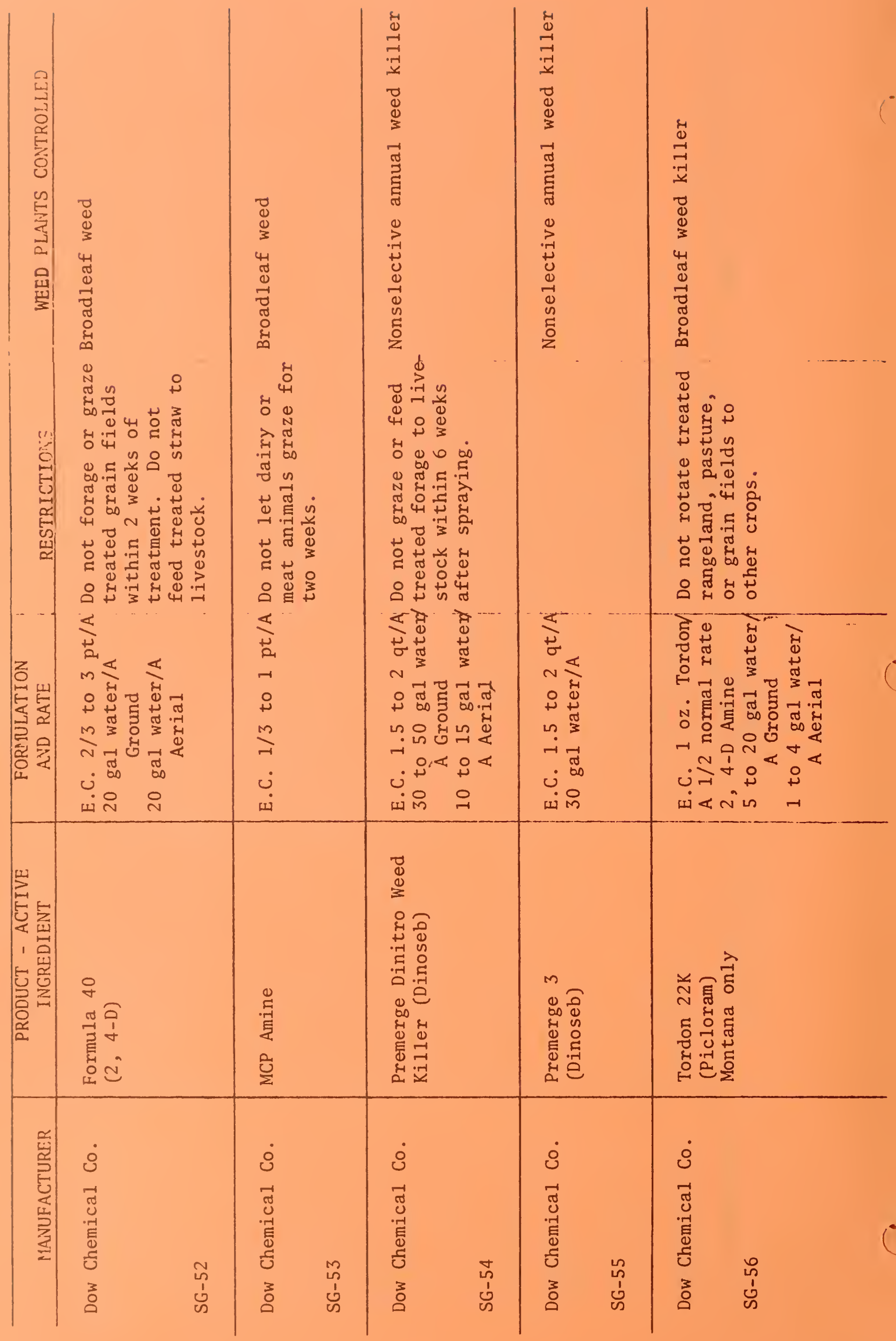




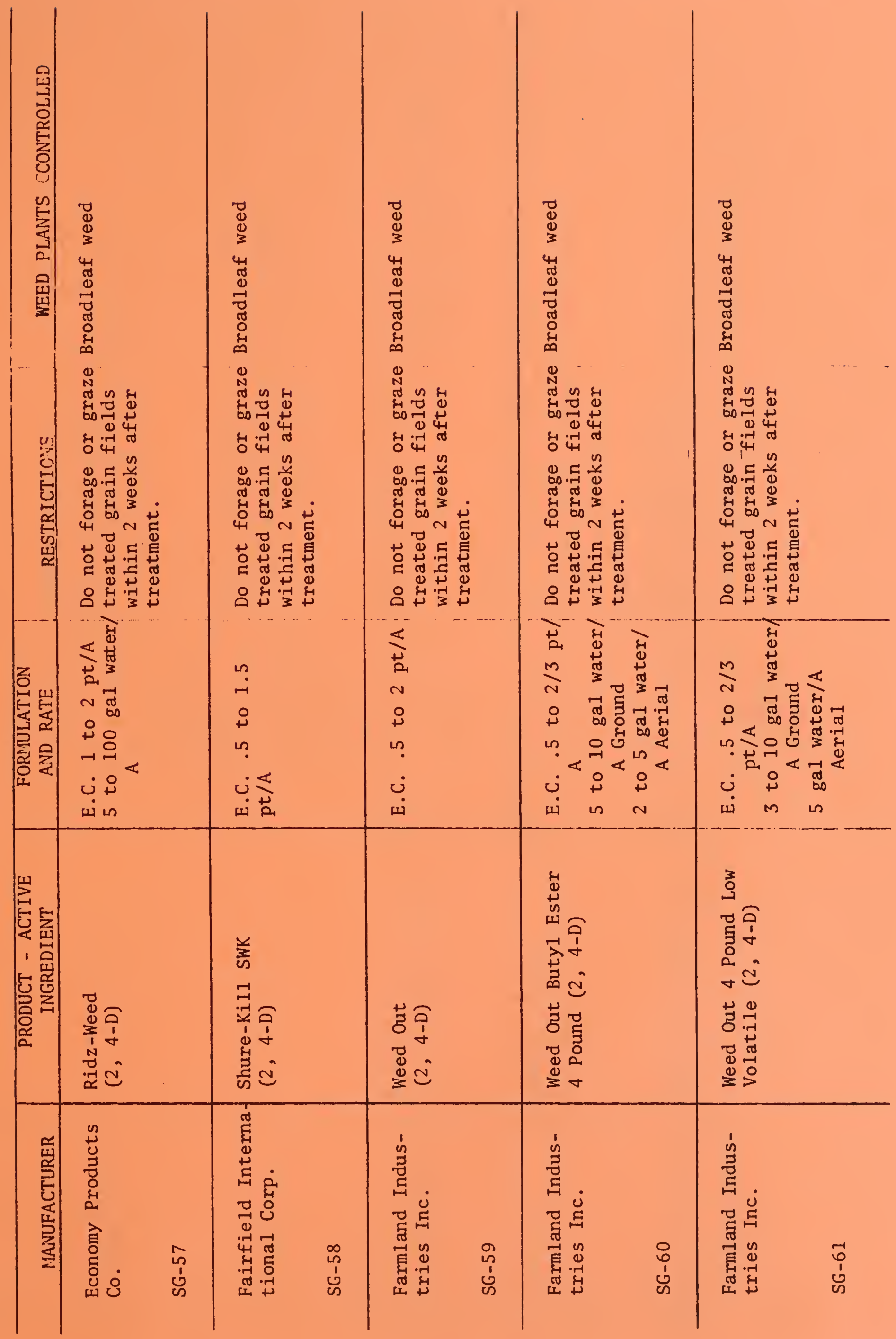




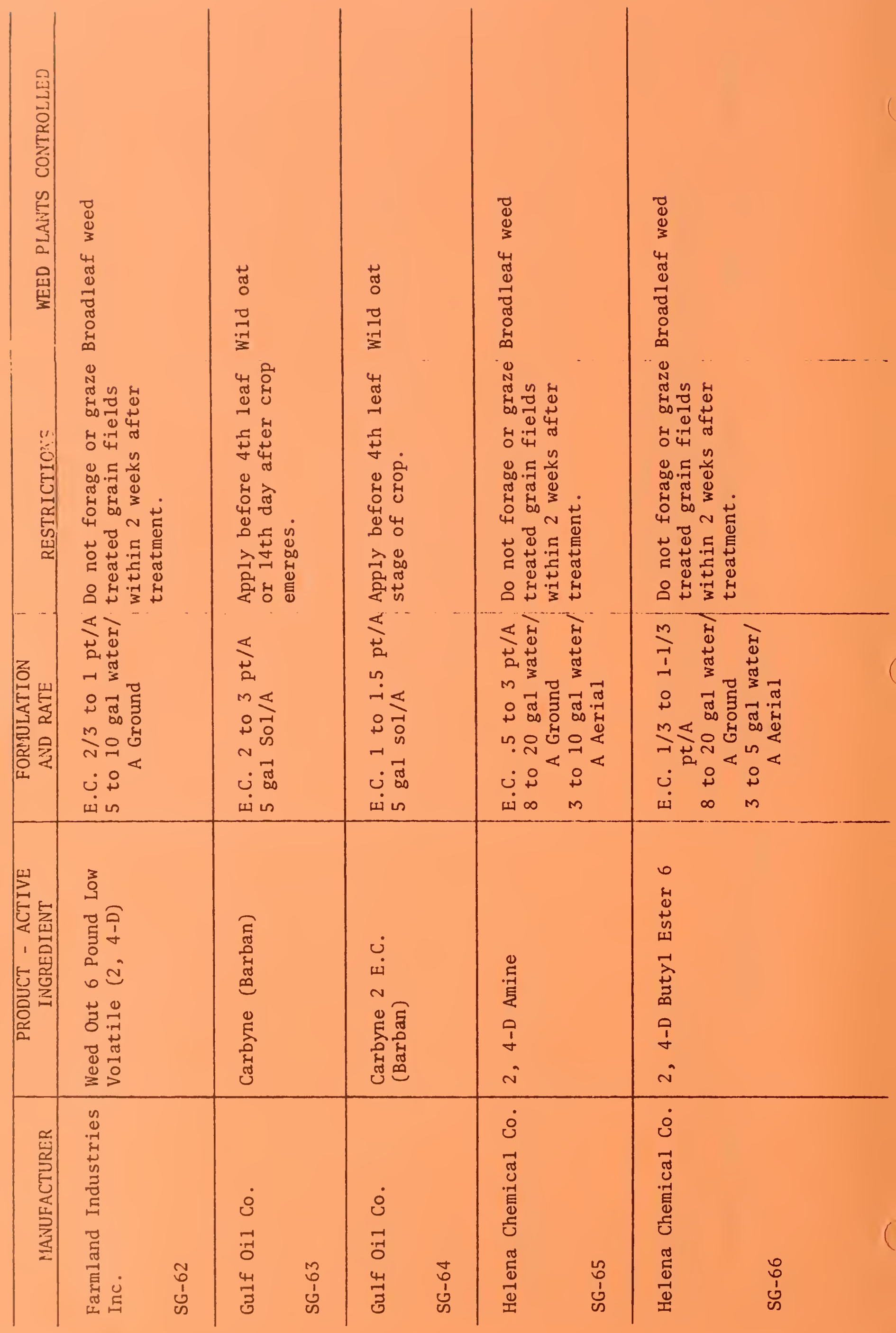




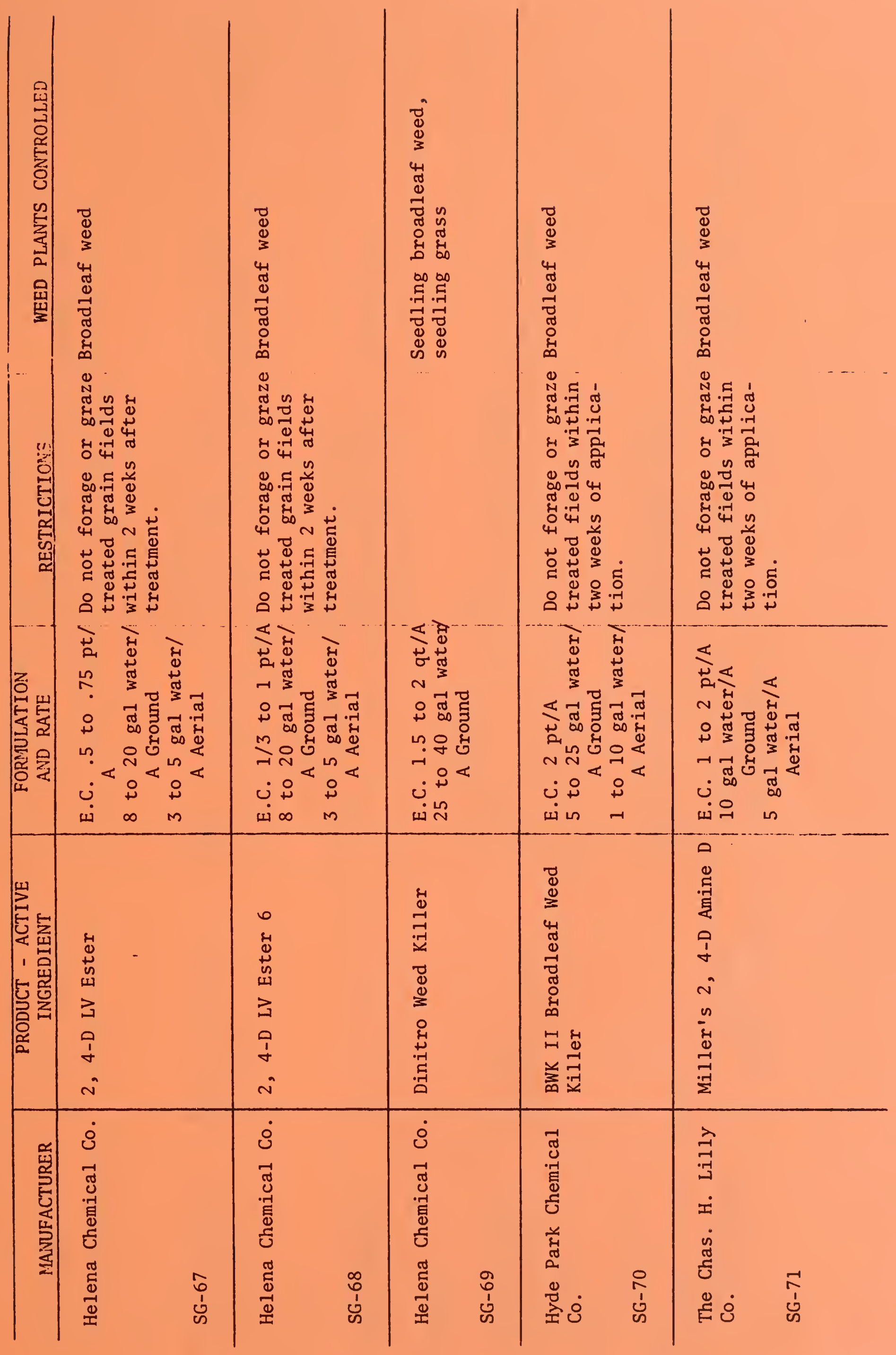




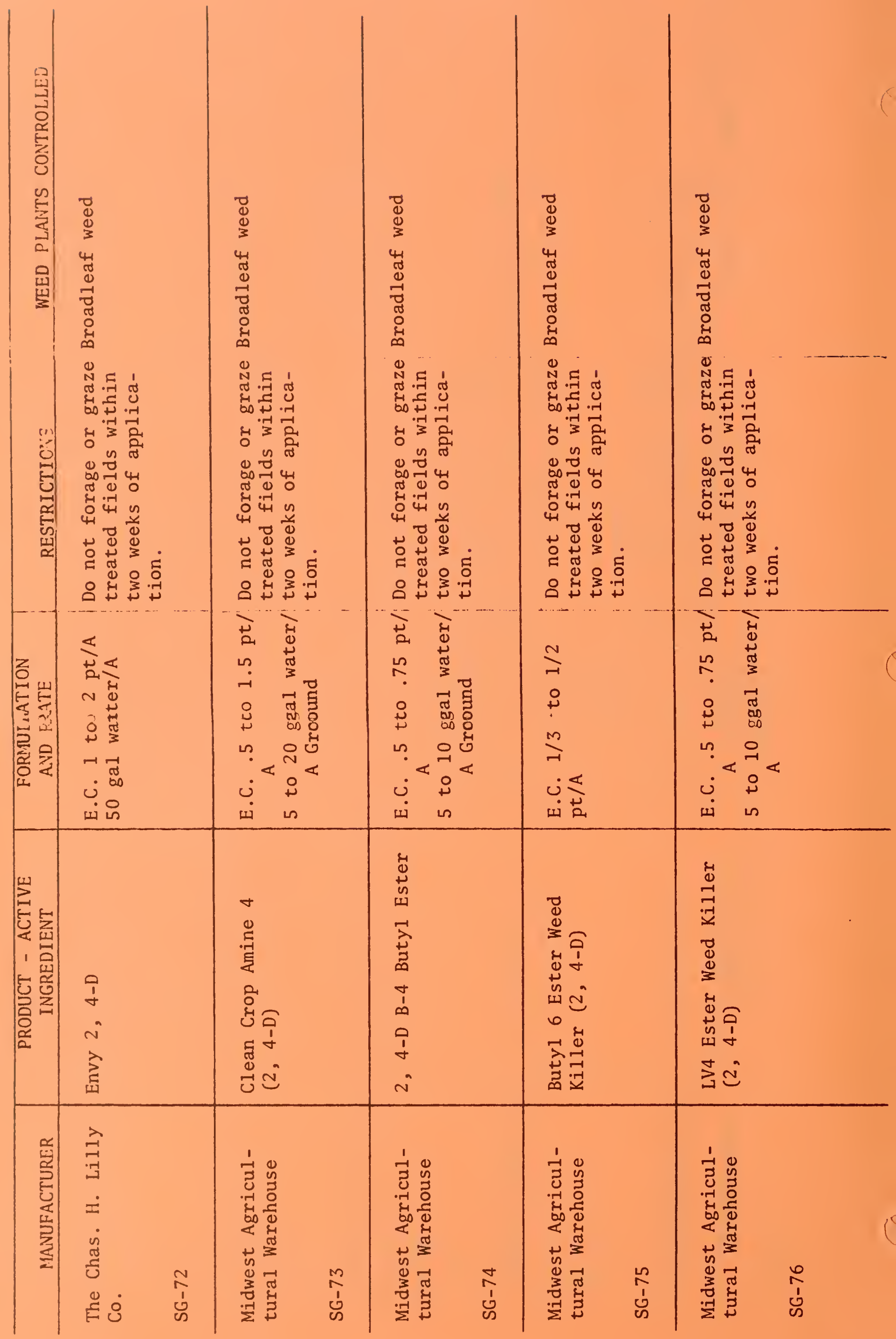




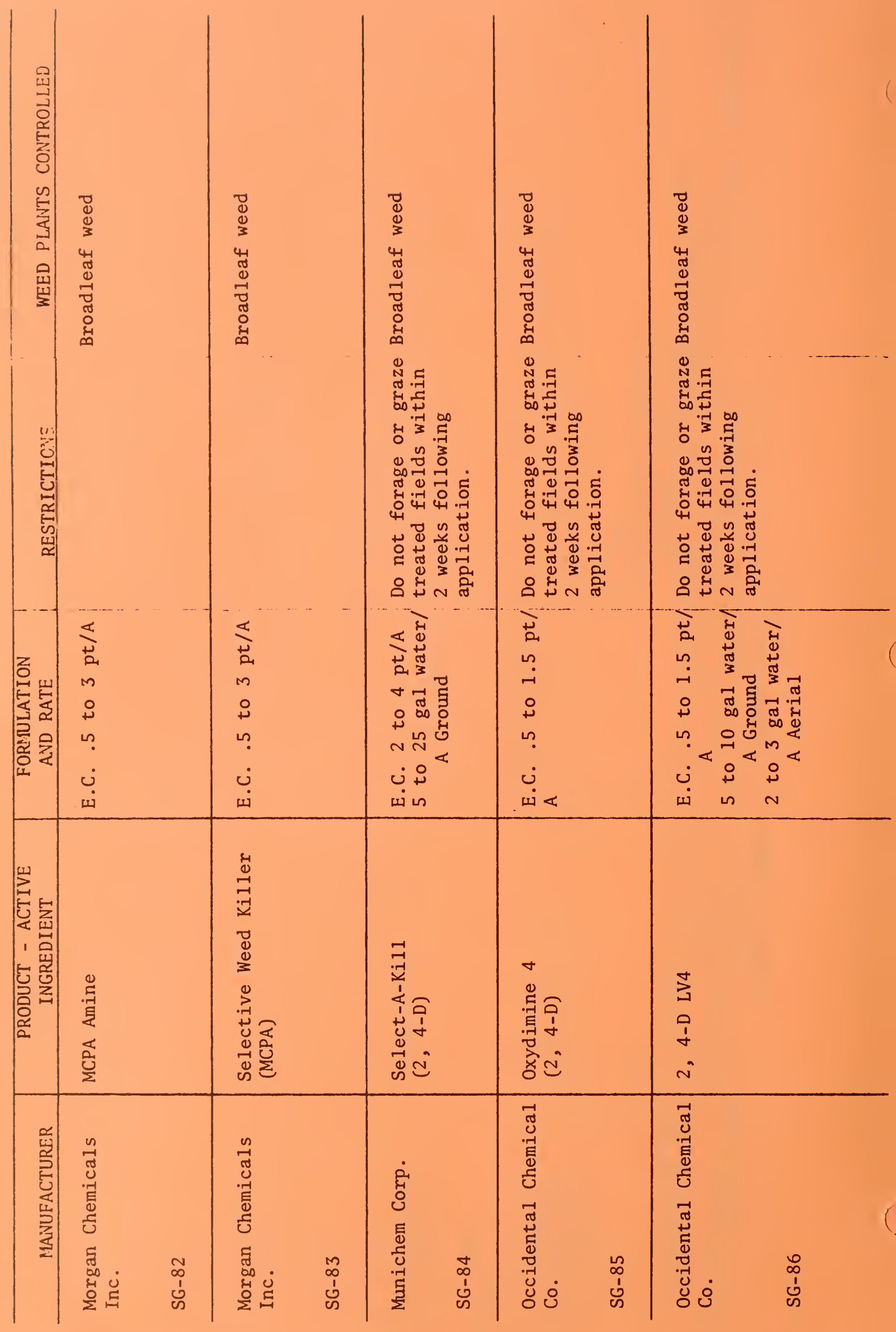




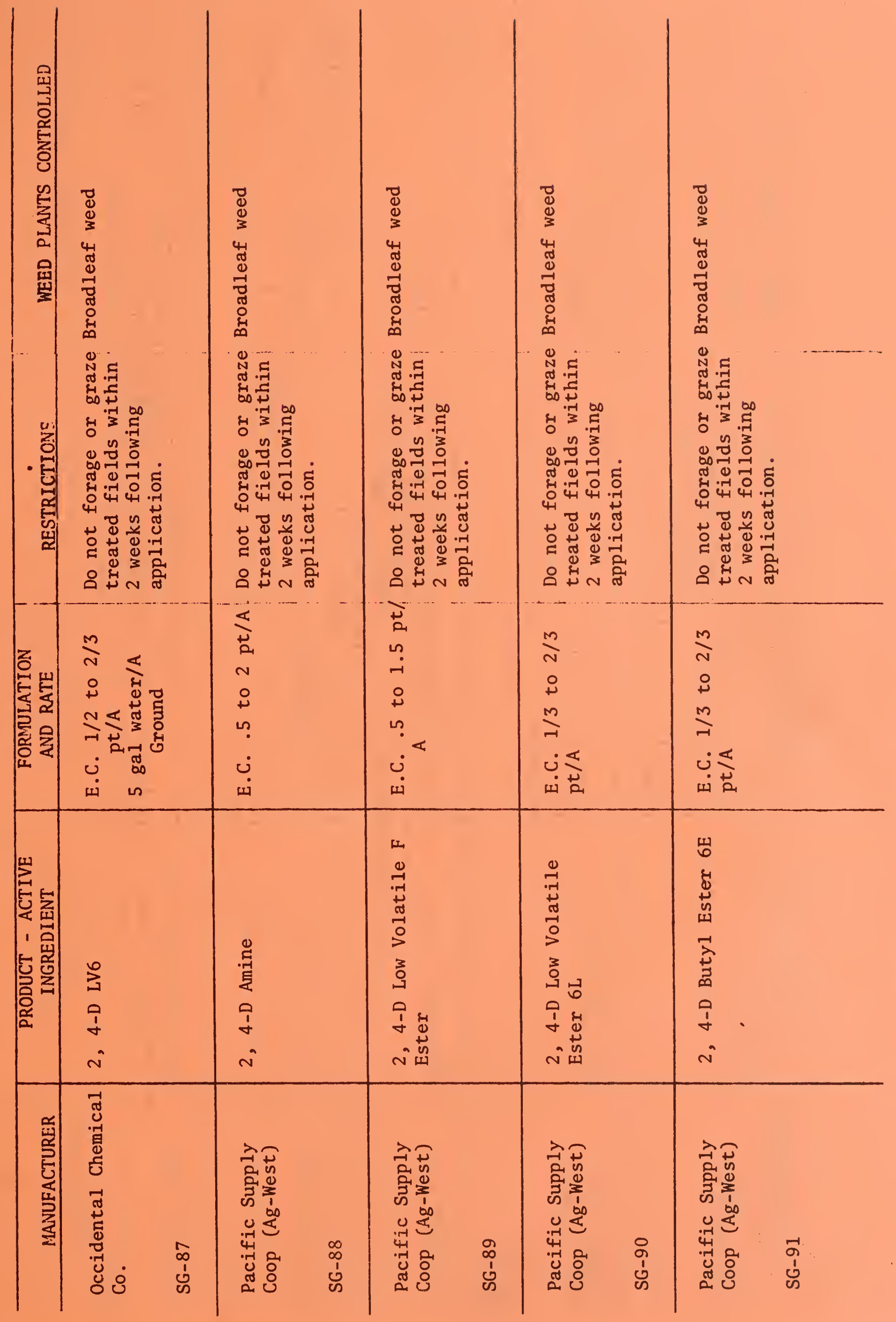




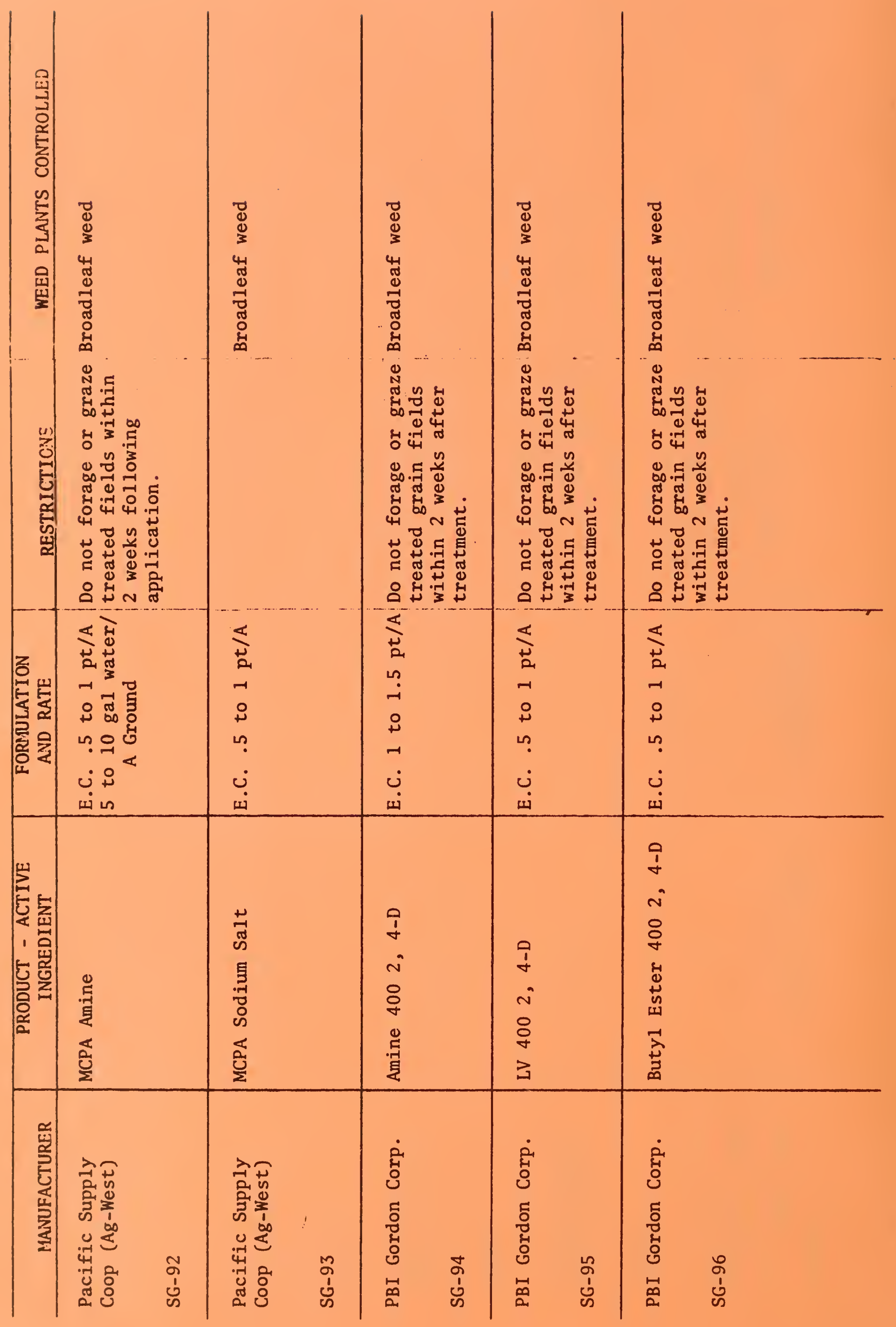




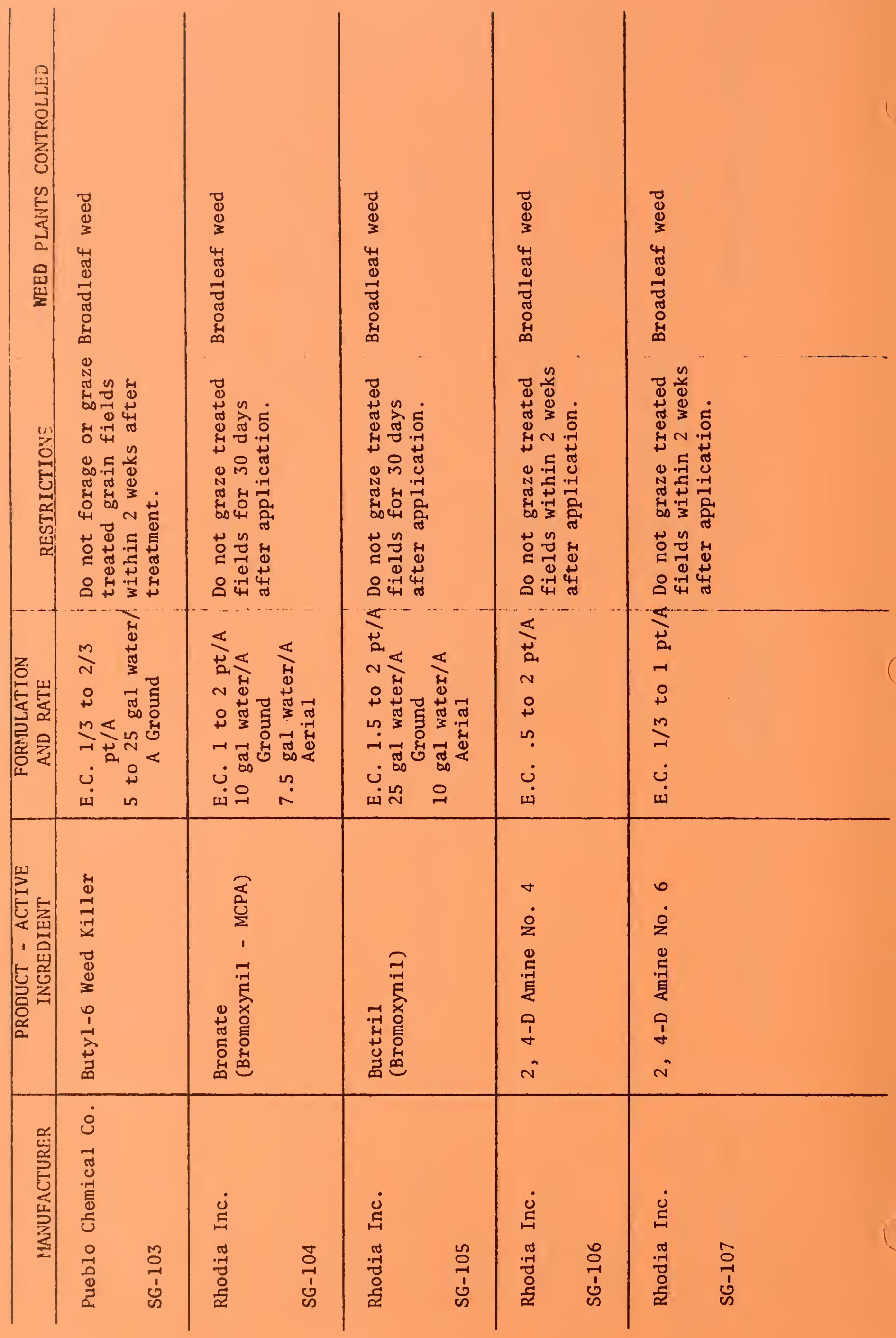




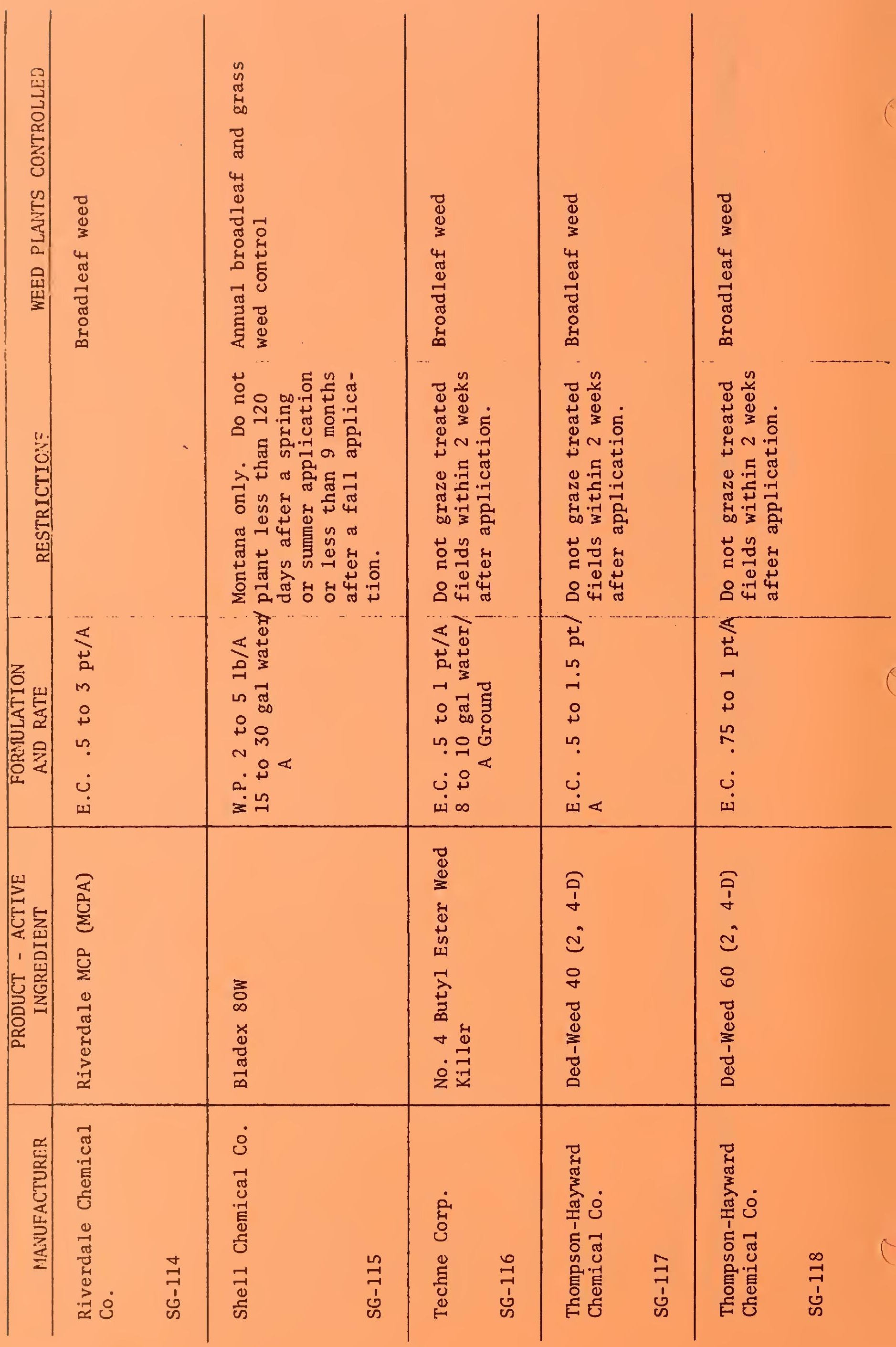




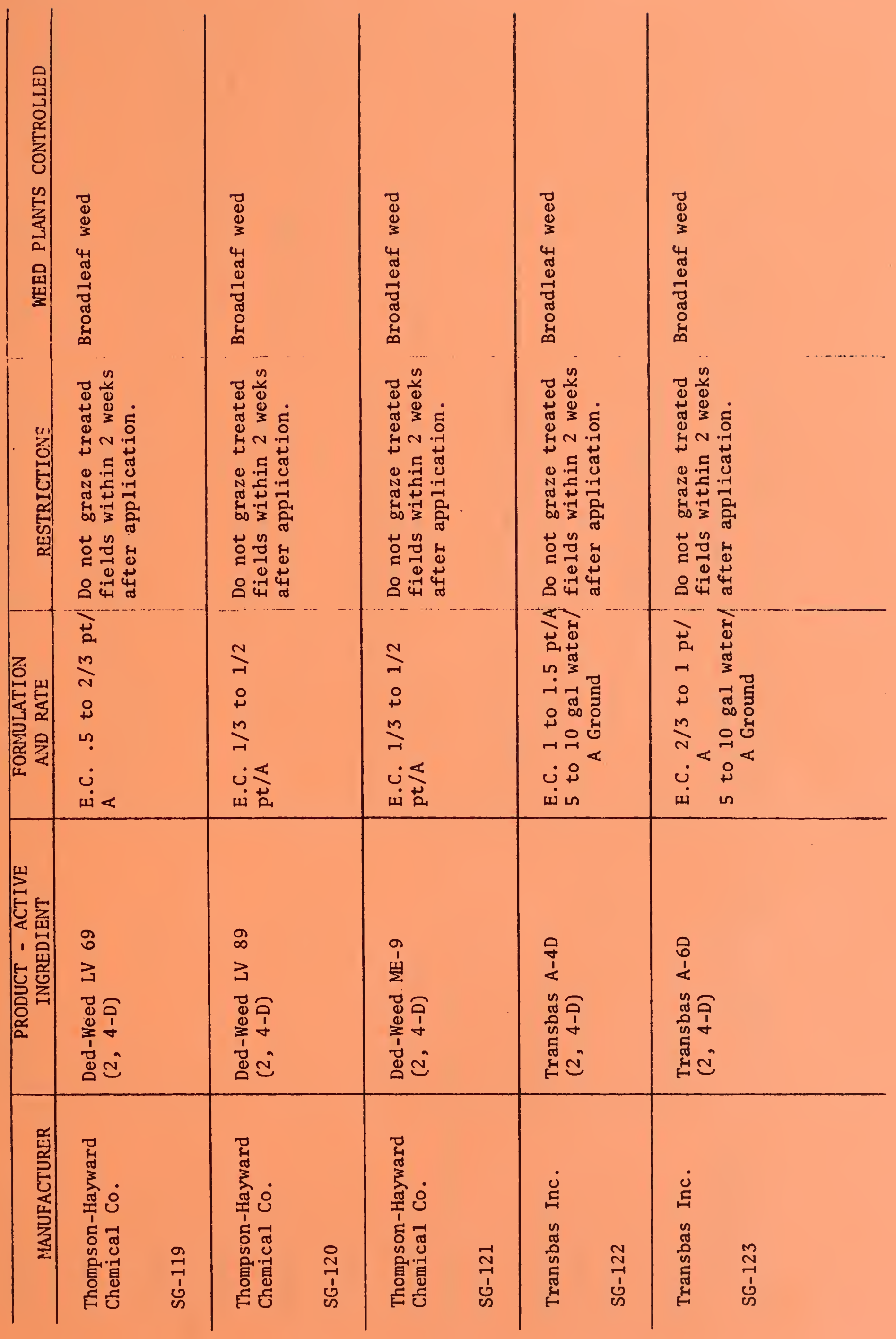




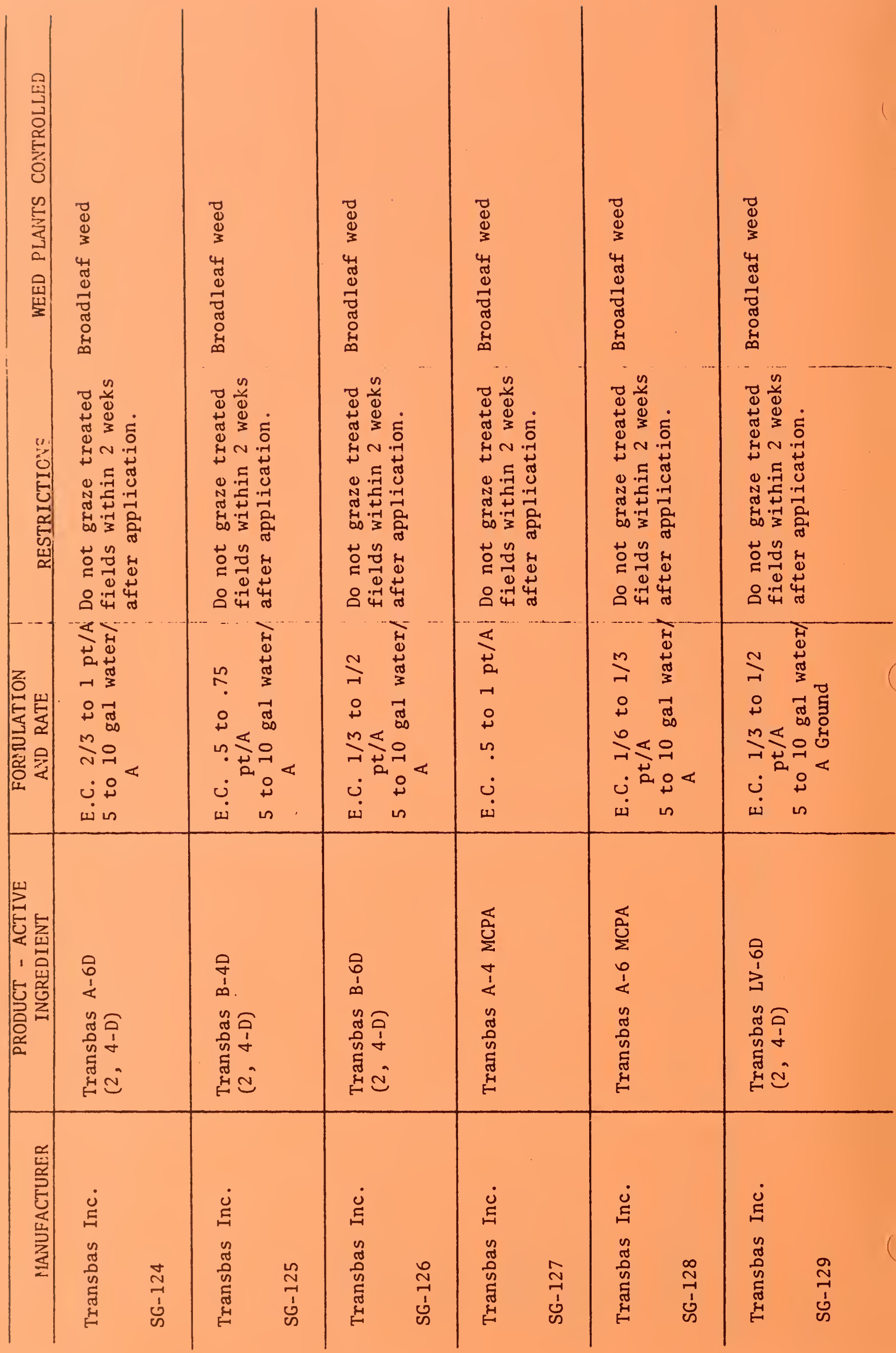




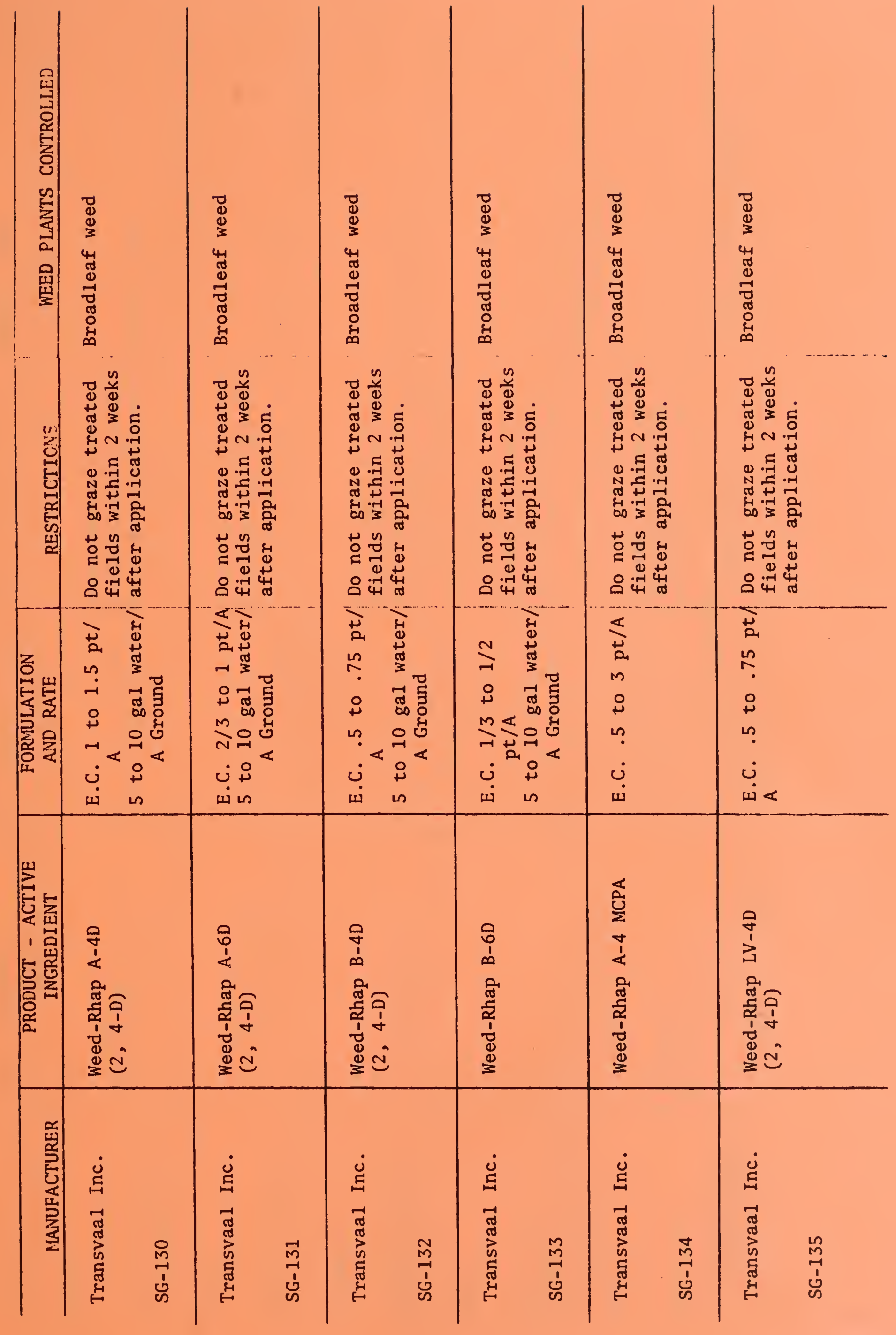




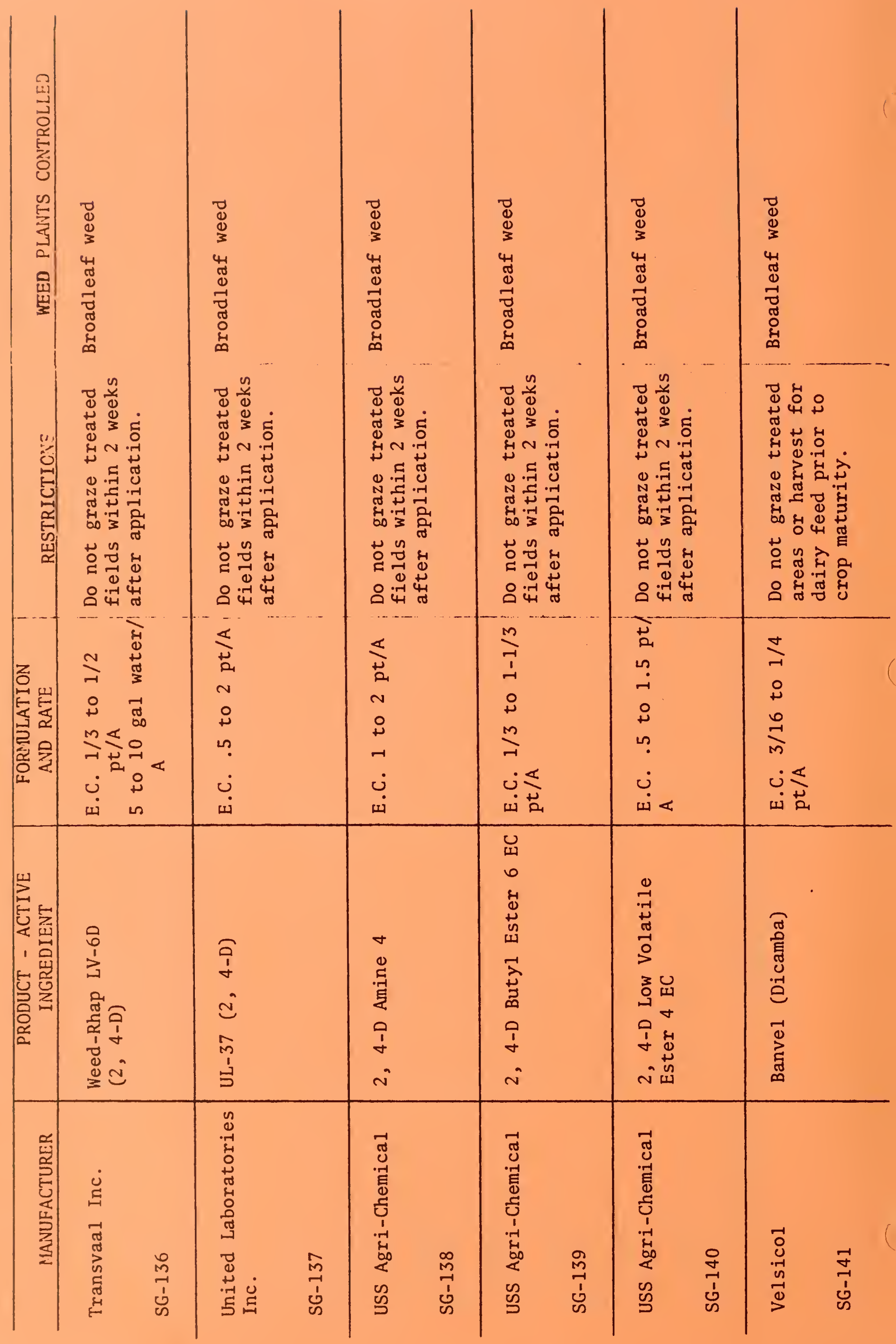




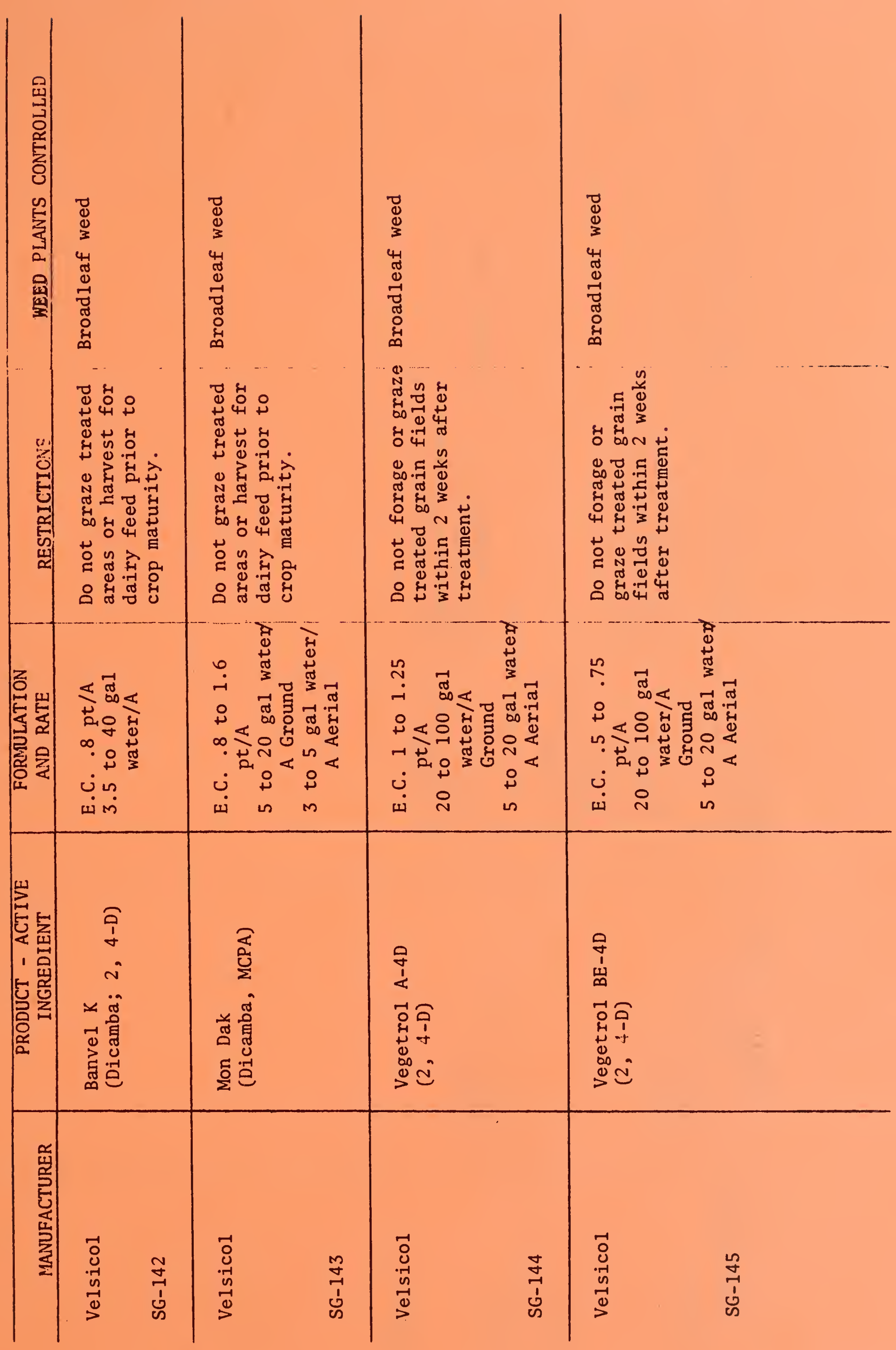




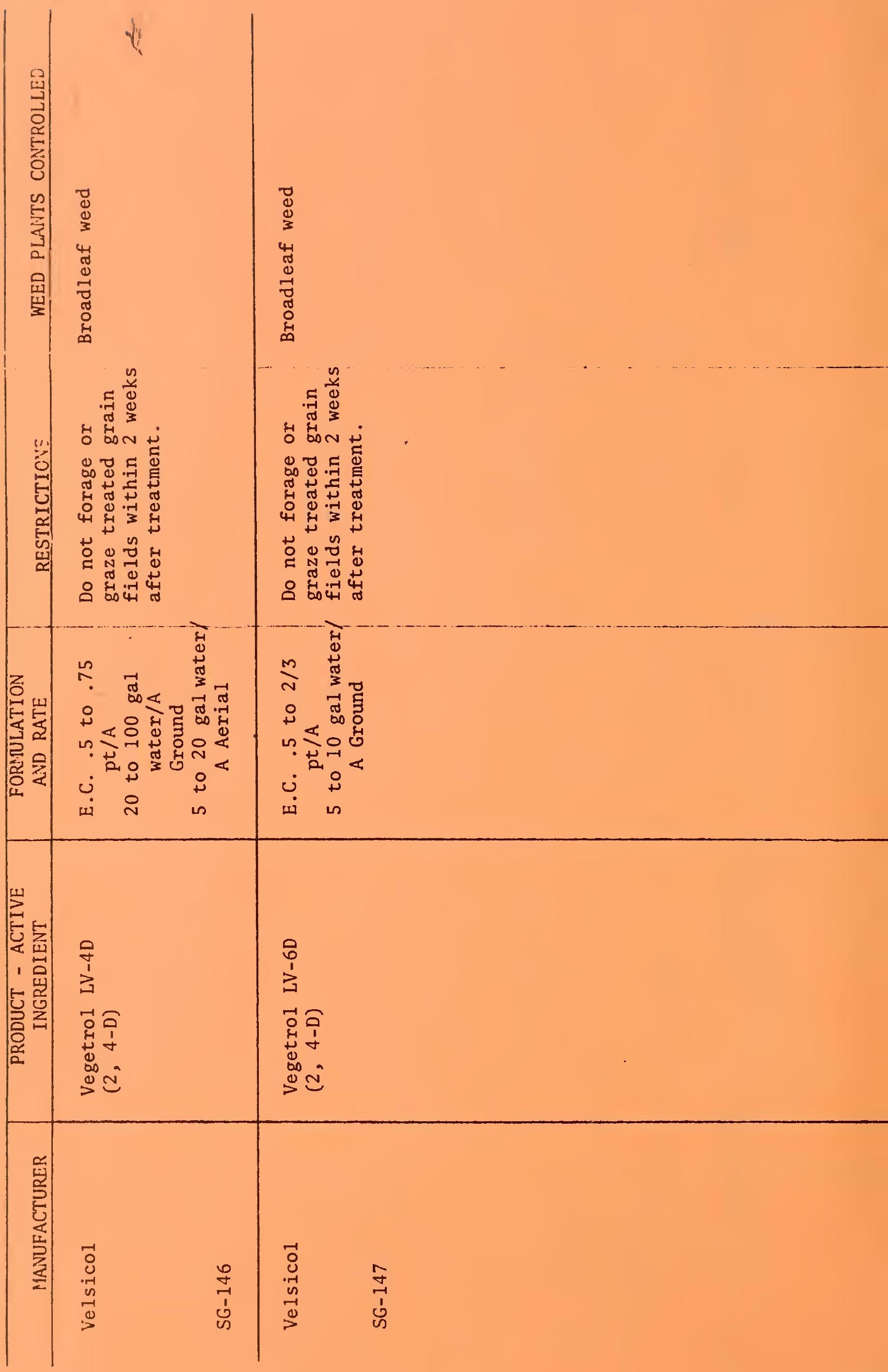





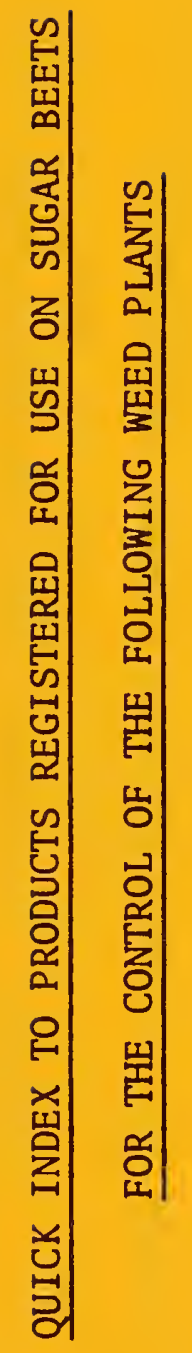

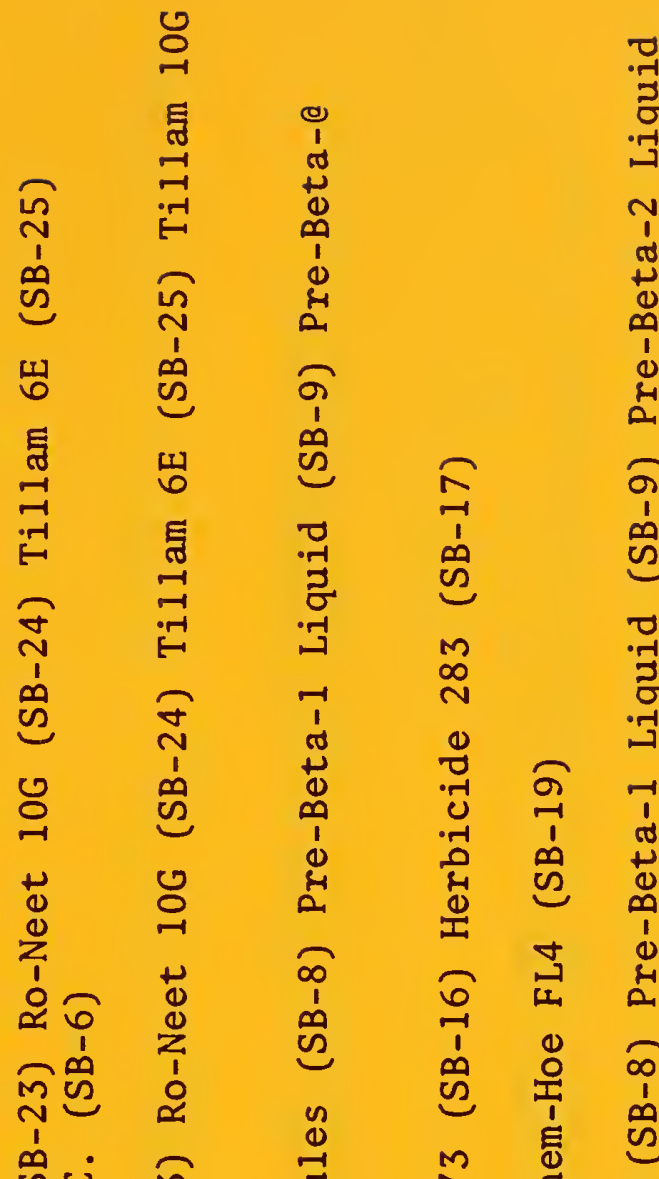

芑

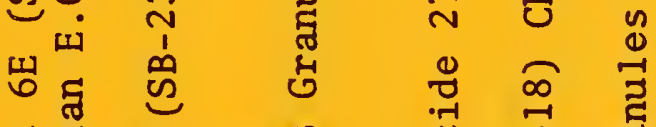

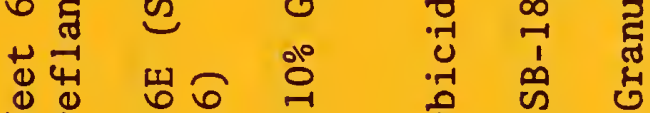

菲

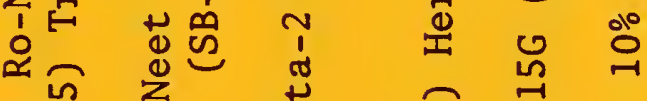

กิ

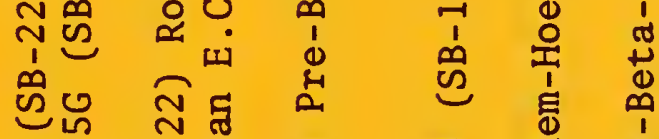

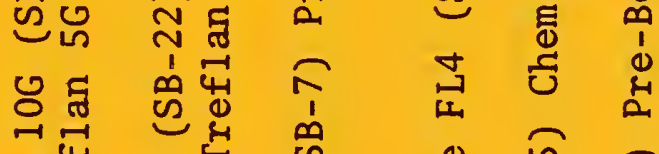

㟧

苛出

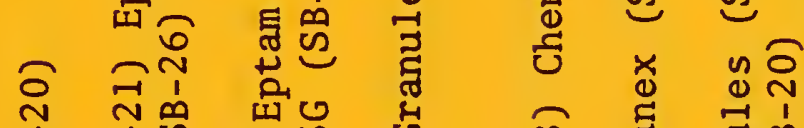

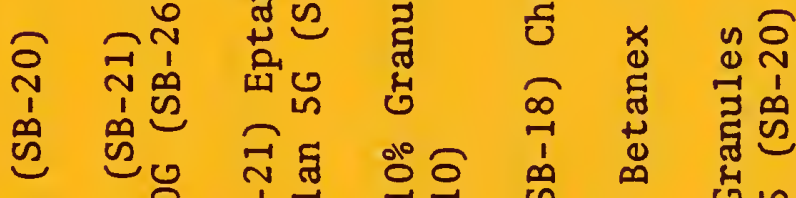

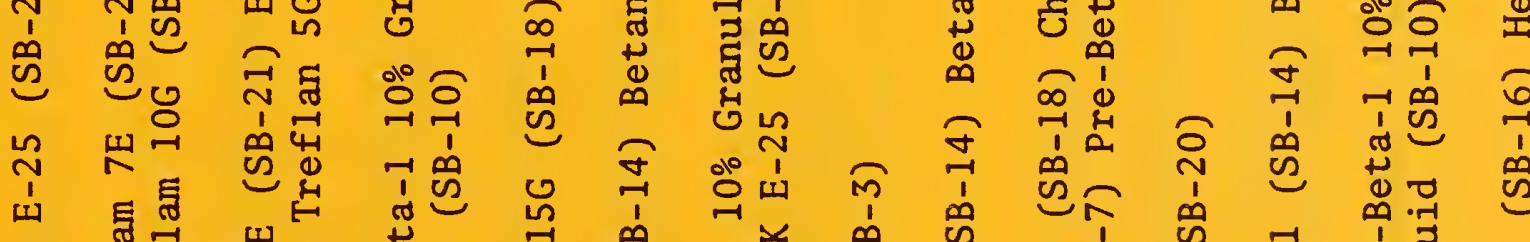
弟䓵寻 零

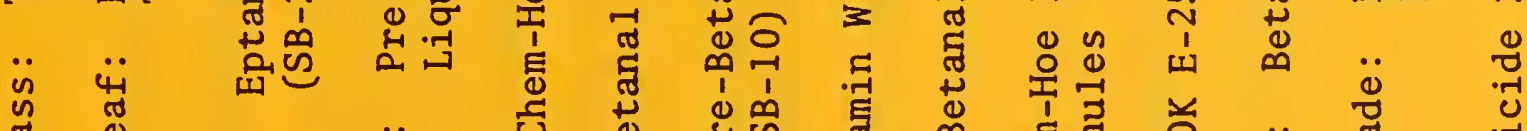

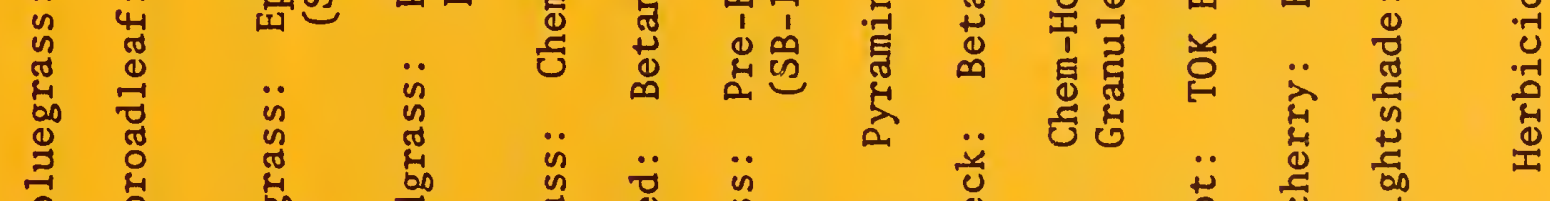

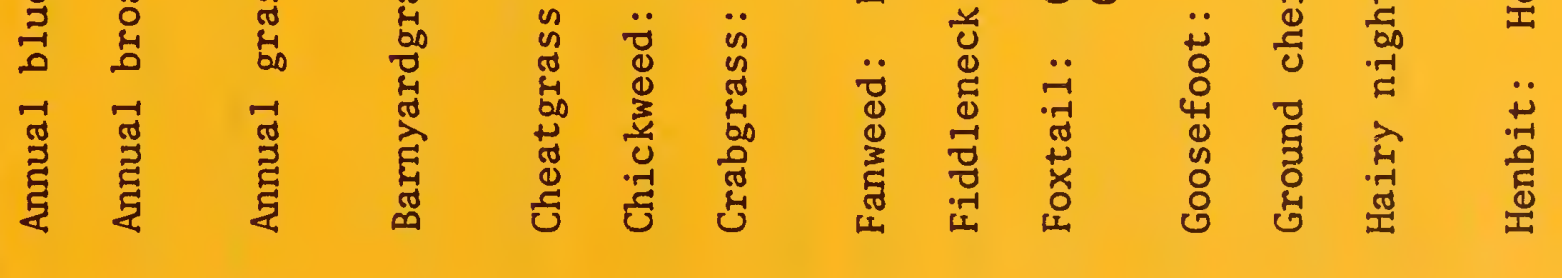




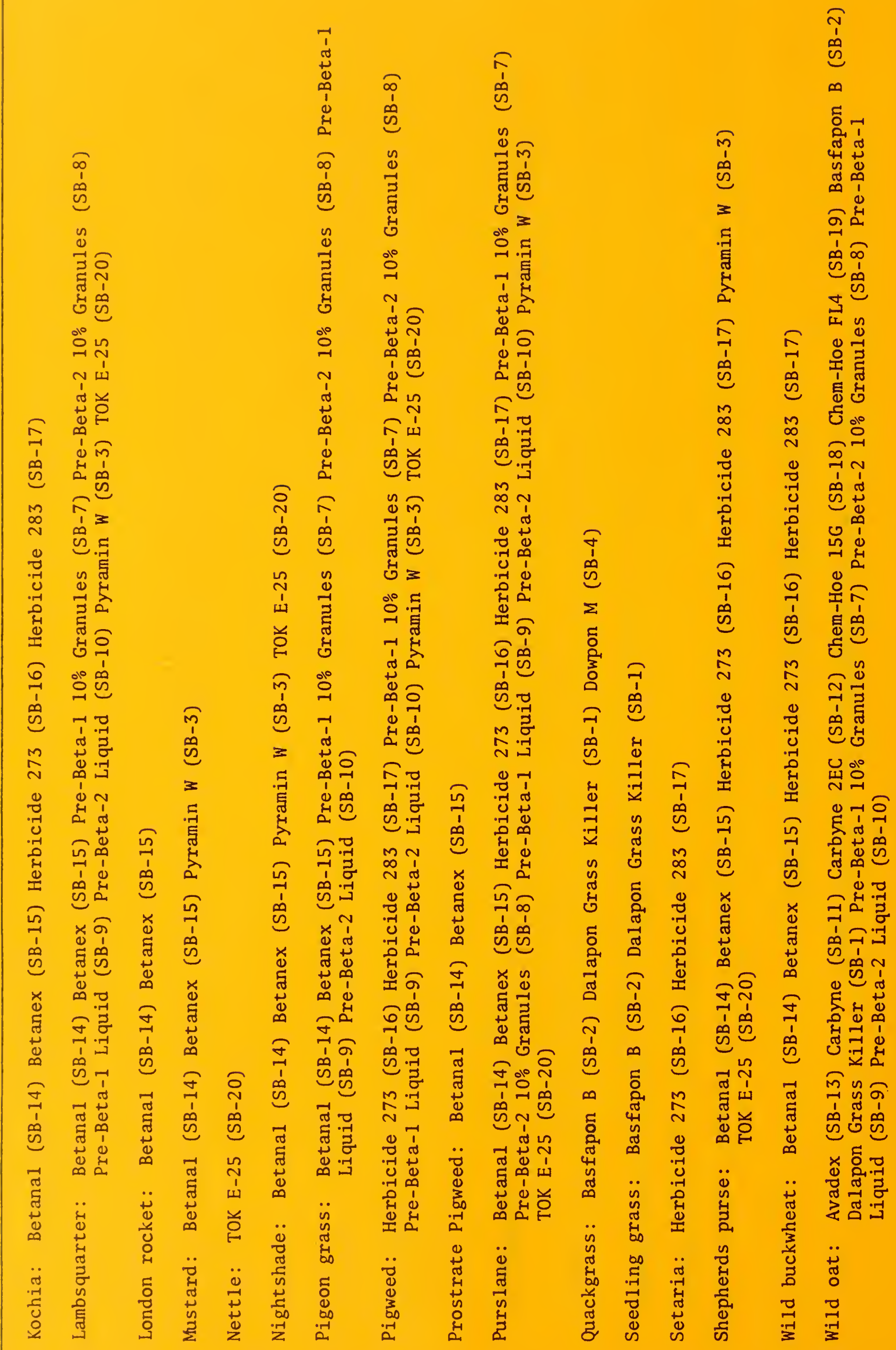




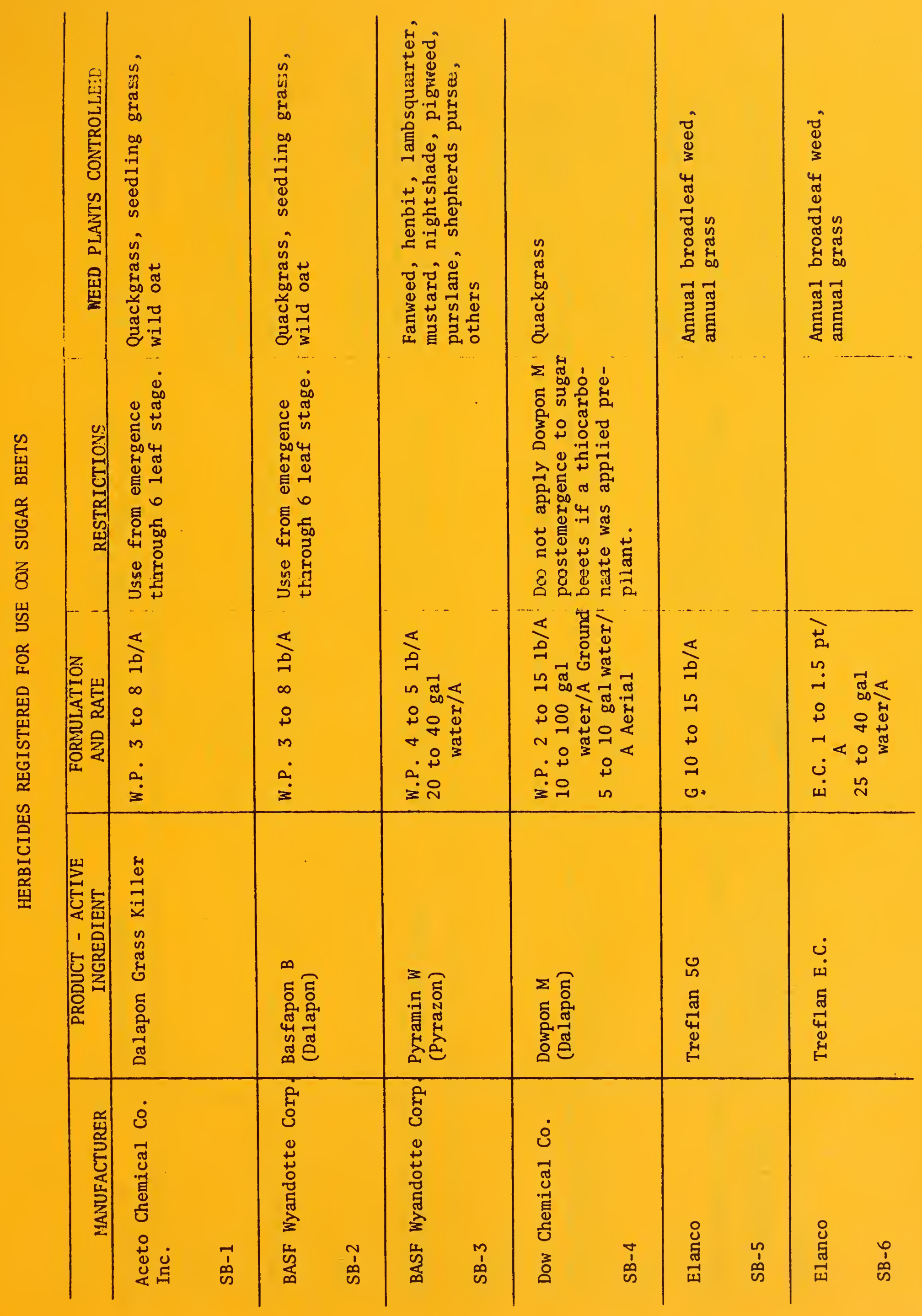




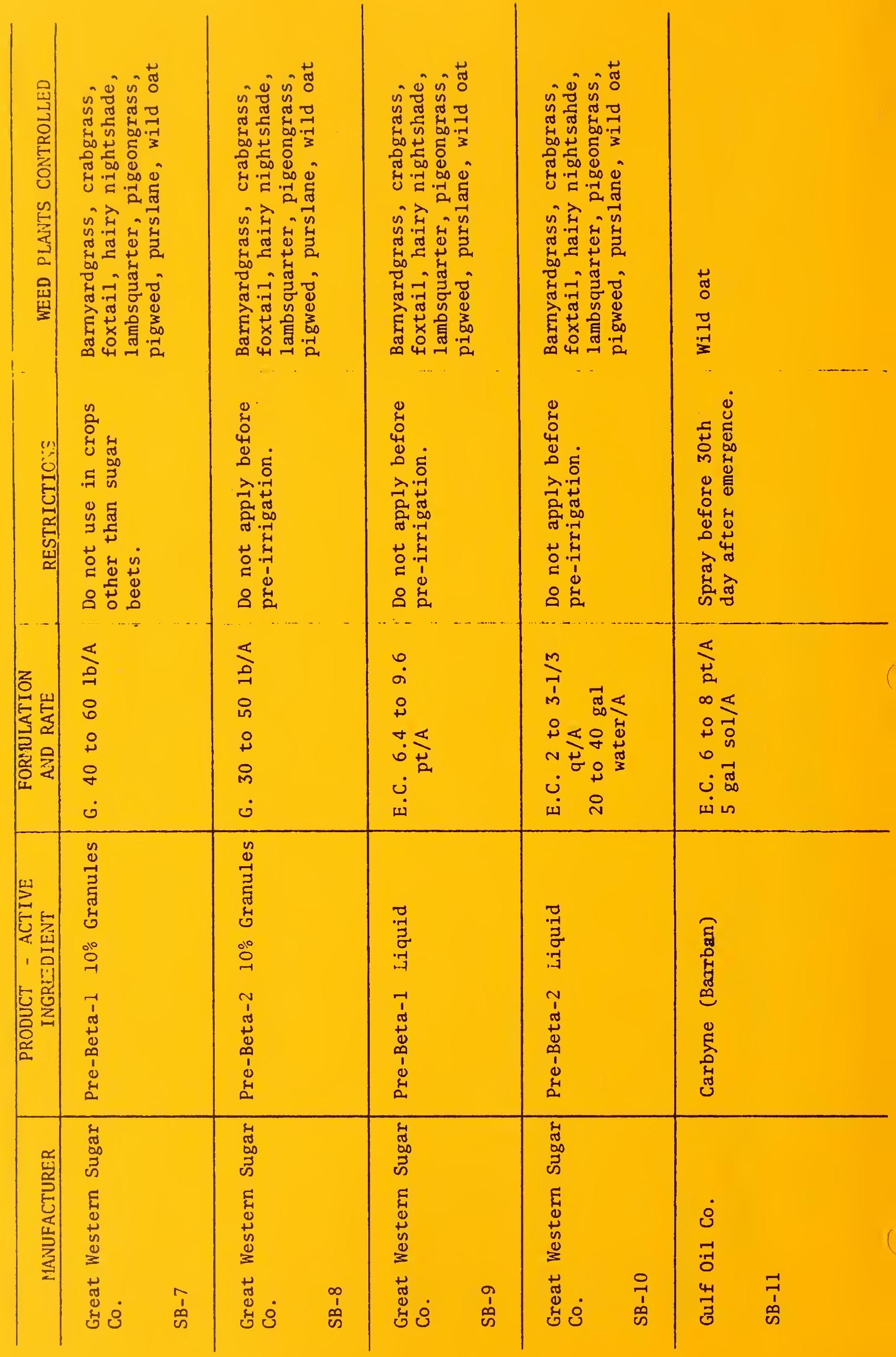




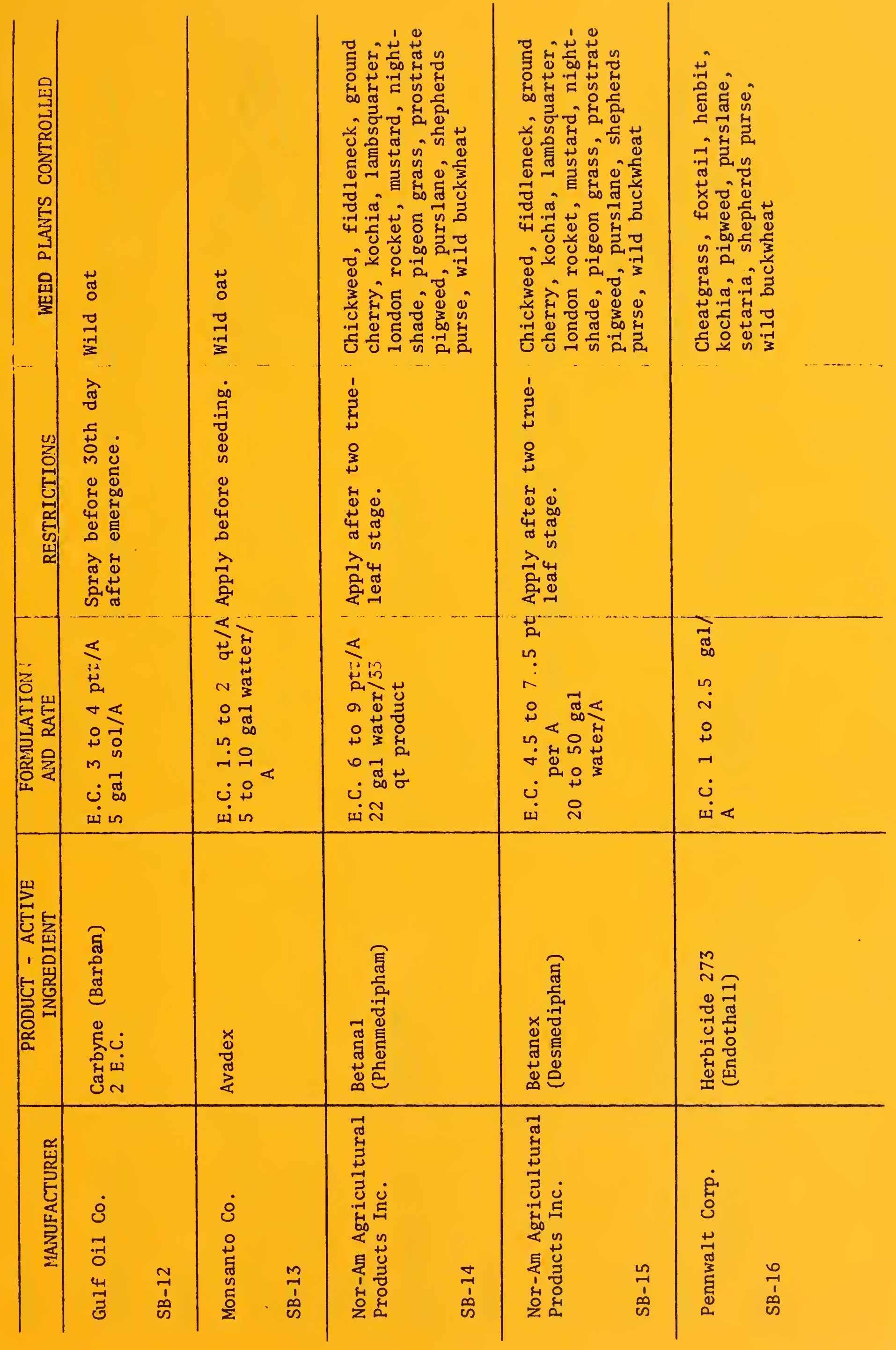




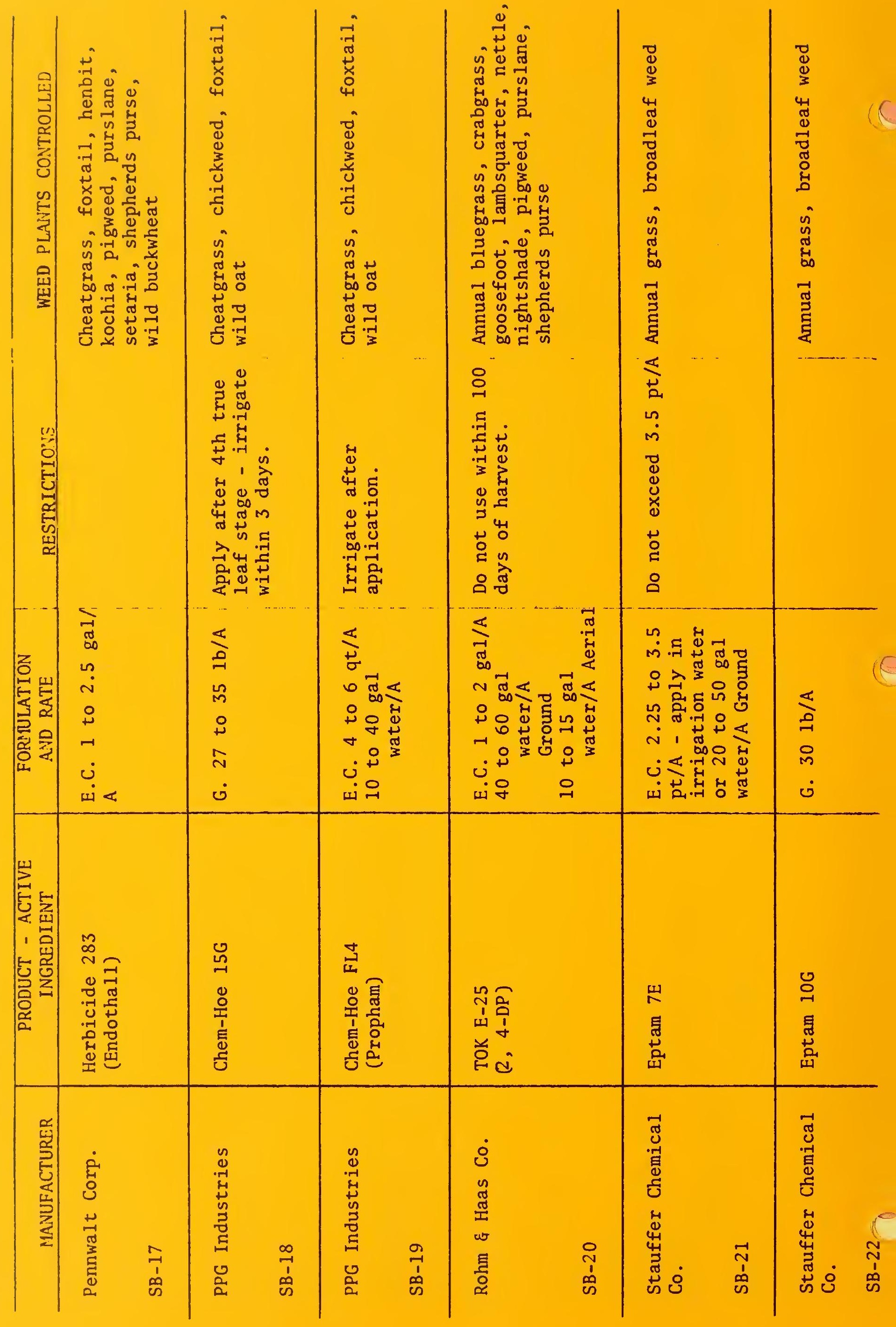




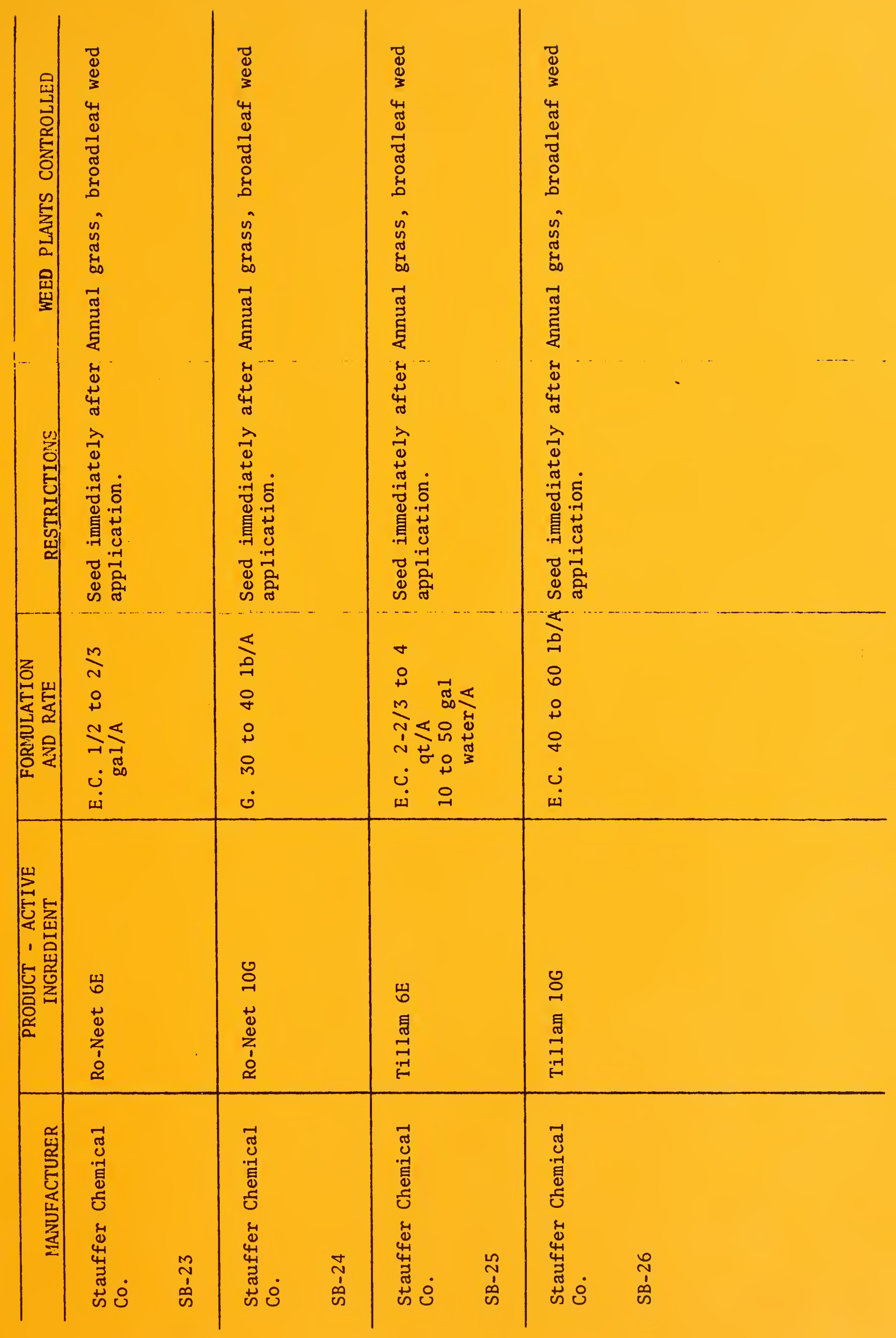






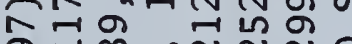

กิ

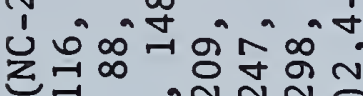
< n na

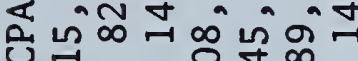

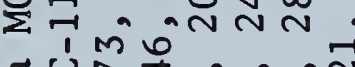
กี้ 实, Uี $N m^{n} N N$ N 近 ช $\mathrm{N}^{\mathrm{N} N}$ क ก้น กล กั Unm ปั H L กูบ オ N ๘ 目 Uु $m$ an $N$. ดั IN $\stackrel{\infty}{N}$ vo

จ듀 ข Z त

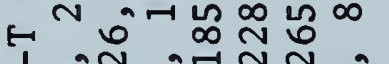

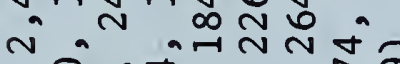

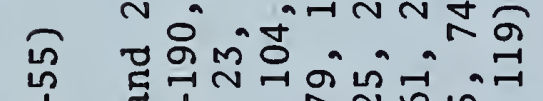

ฮี่

ด \& N 岂 ก $\Sigma$ an की 寻穴 . $\mathrm{N}$ a a a a is 1 $\sim$ in $\infty$ N u 의 ก Z,${ }^{\prime} \sim N$ N

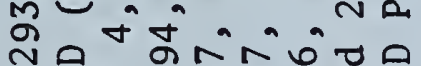
บ่ Z ๓ 䓍ด ๙ 帠势

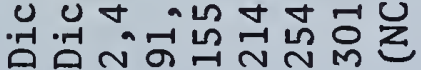<smiles>[C-]#[Hg]</smiles>
萑 ब म न

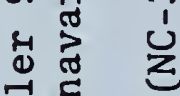
덩 的 8 $\nabla 0$

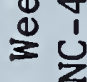
z

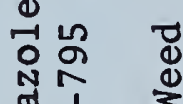
तो 年 0 ल ल O 娦艺 ก 艺 是 ก
U
$z$ ㅇำ 尔 $\begin{array}{lll}z & 0 & \text { in } \\ 0 & 0 & 0\end{array}$

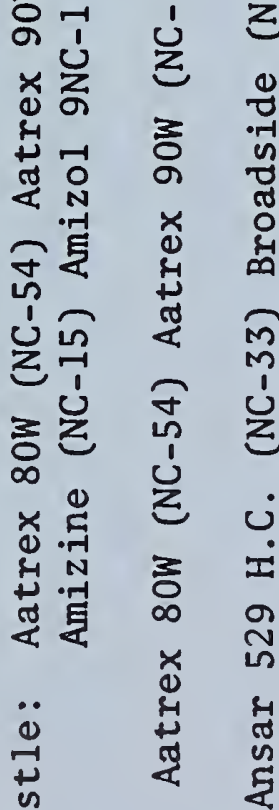

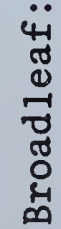




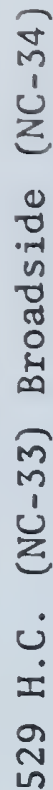

मे

ผี ข

延

6

艺管

오은

喪亦

เก

-1

光

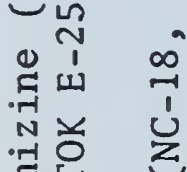

留尔

กิก हี

นก

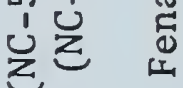

증요

ดั

$\times$ 告

茫

岁完

ช

मे द

㲻交

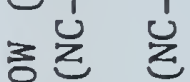

×

焉

焉志志

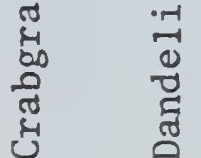

몬

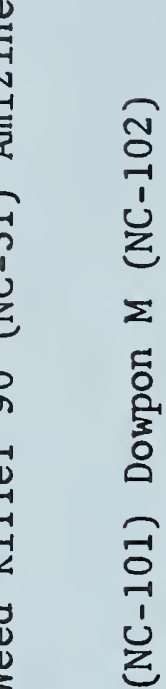

$\stackrel{n}{1}$

음

ล

ब

ঠָ

¿

票

$\Sigma$

్ㅗㅇ

얼

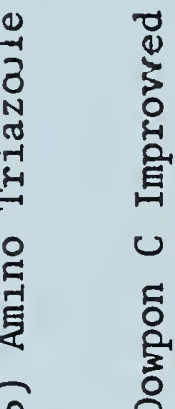

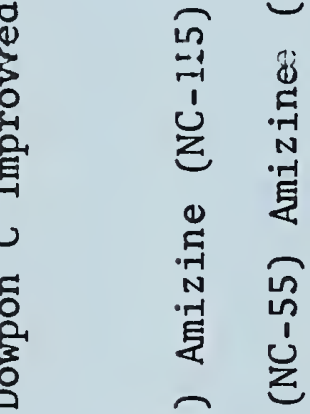

ฮิ

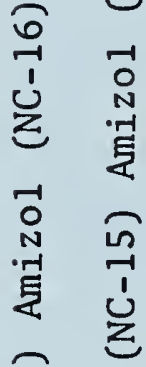

苑 离

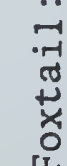

8
7
ப'

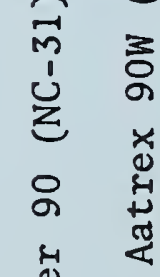

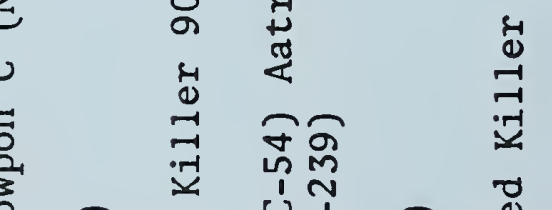

สิ $)$ ป

立

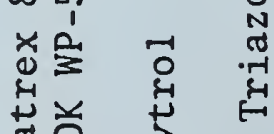

हี

$\ddot{0}$

芯韋

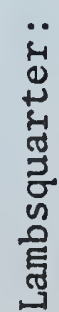

$\infty$ 芹

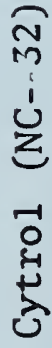

ชู่

चैन्न -

엄ำ

- Ñ்

कलㅡㅁำ

กं

$\infty$ กิ

N

in in

$M-\pi N$

iी an $\infty$ in Mचन

जी बंत्रां

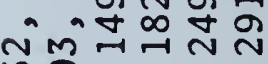

in

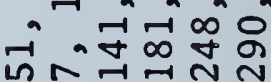
$\infty$ in 0 i̊ $\infty$ - $N$ N की $\infty m+\infty$ $\infty,-\pi N$ \$ $\infty$ in ㅁำ

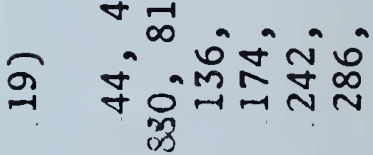

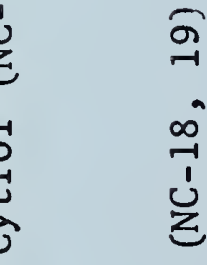

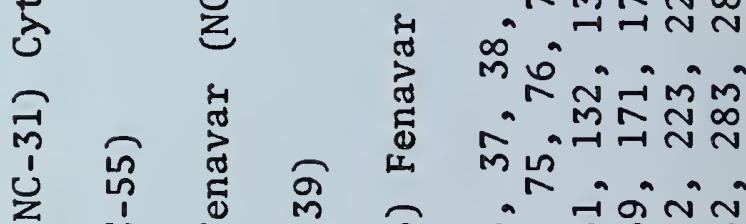
3 U⿺辶⿻

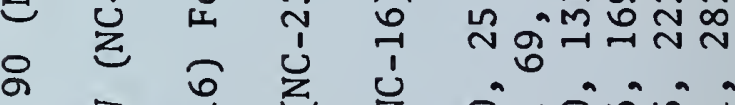
- ₹

a 3 in 党

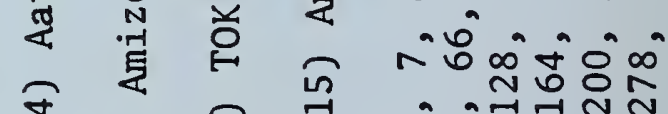
i 2 क 年 I iै Ư⿱ 


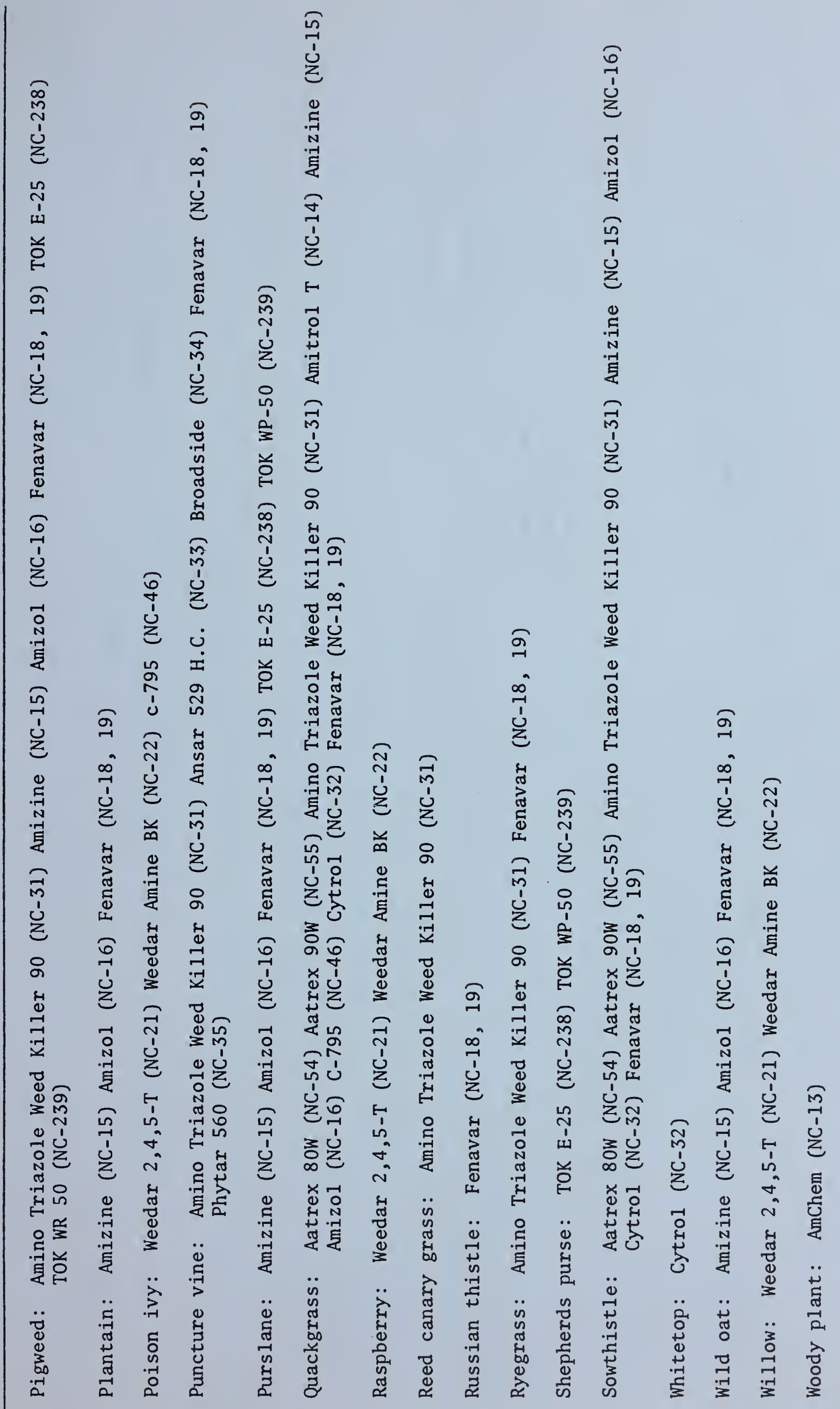




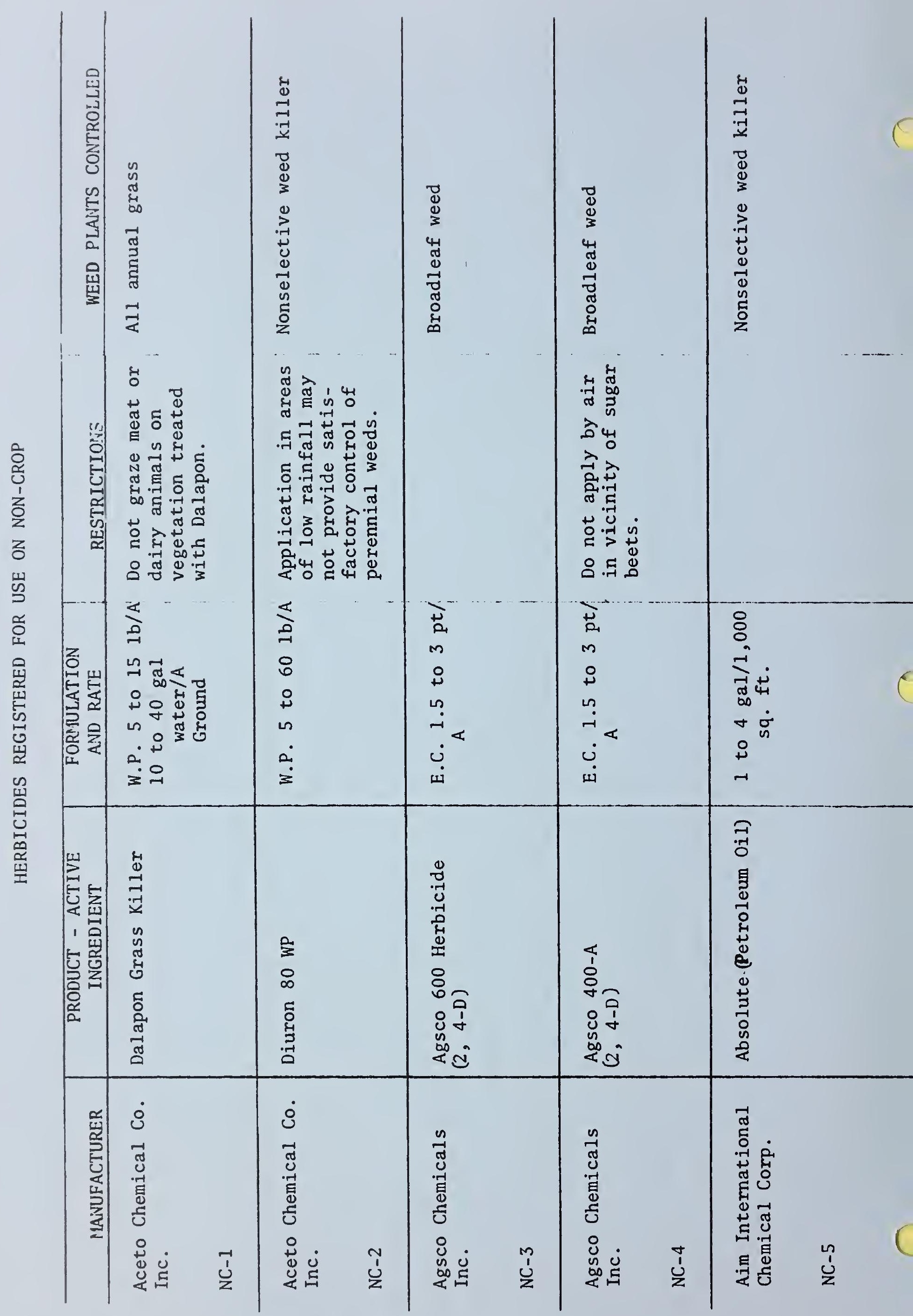




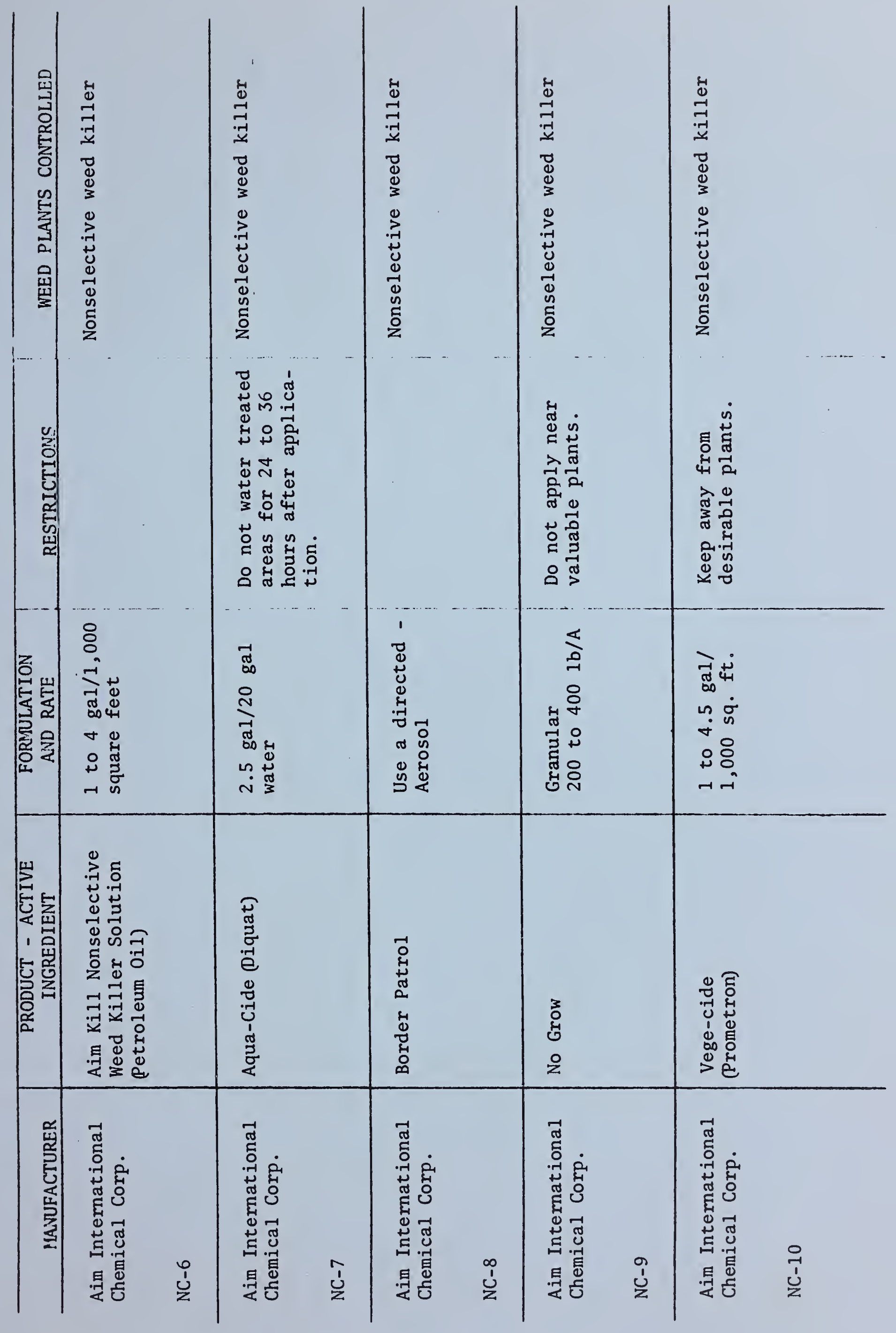




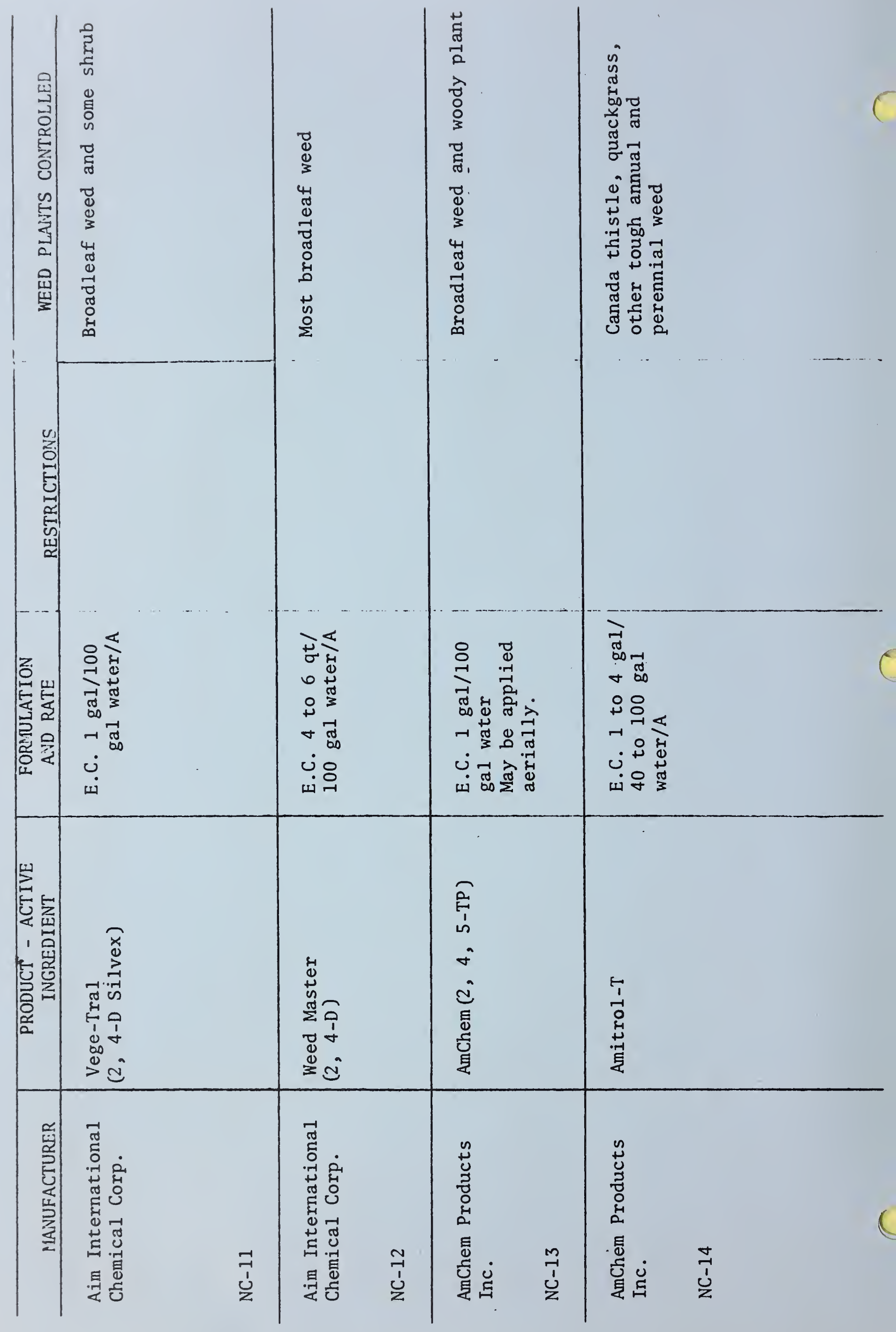




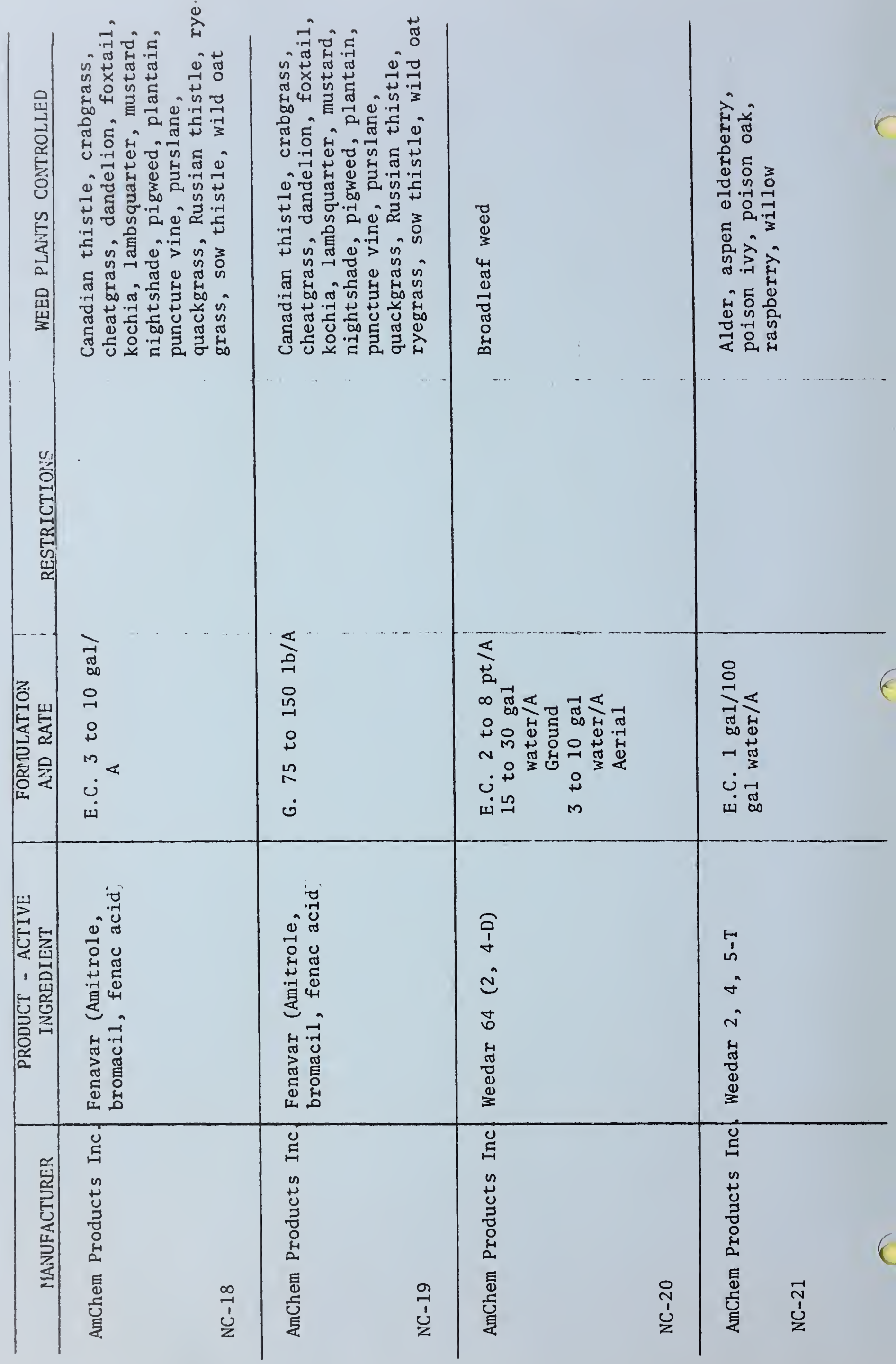




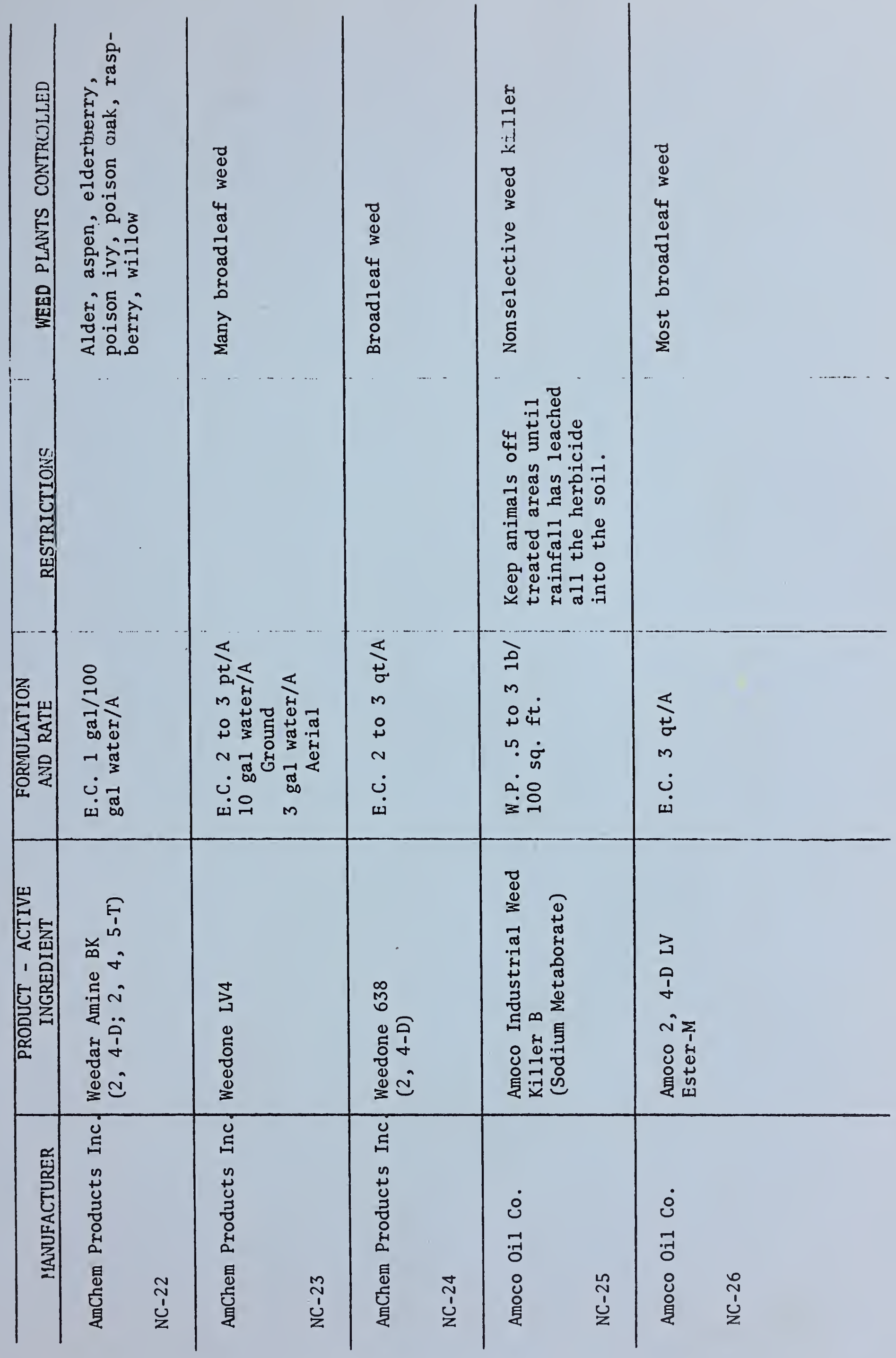




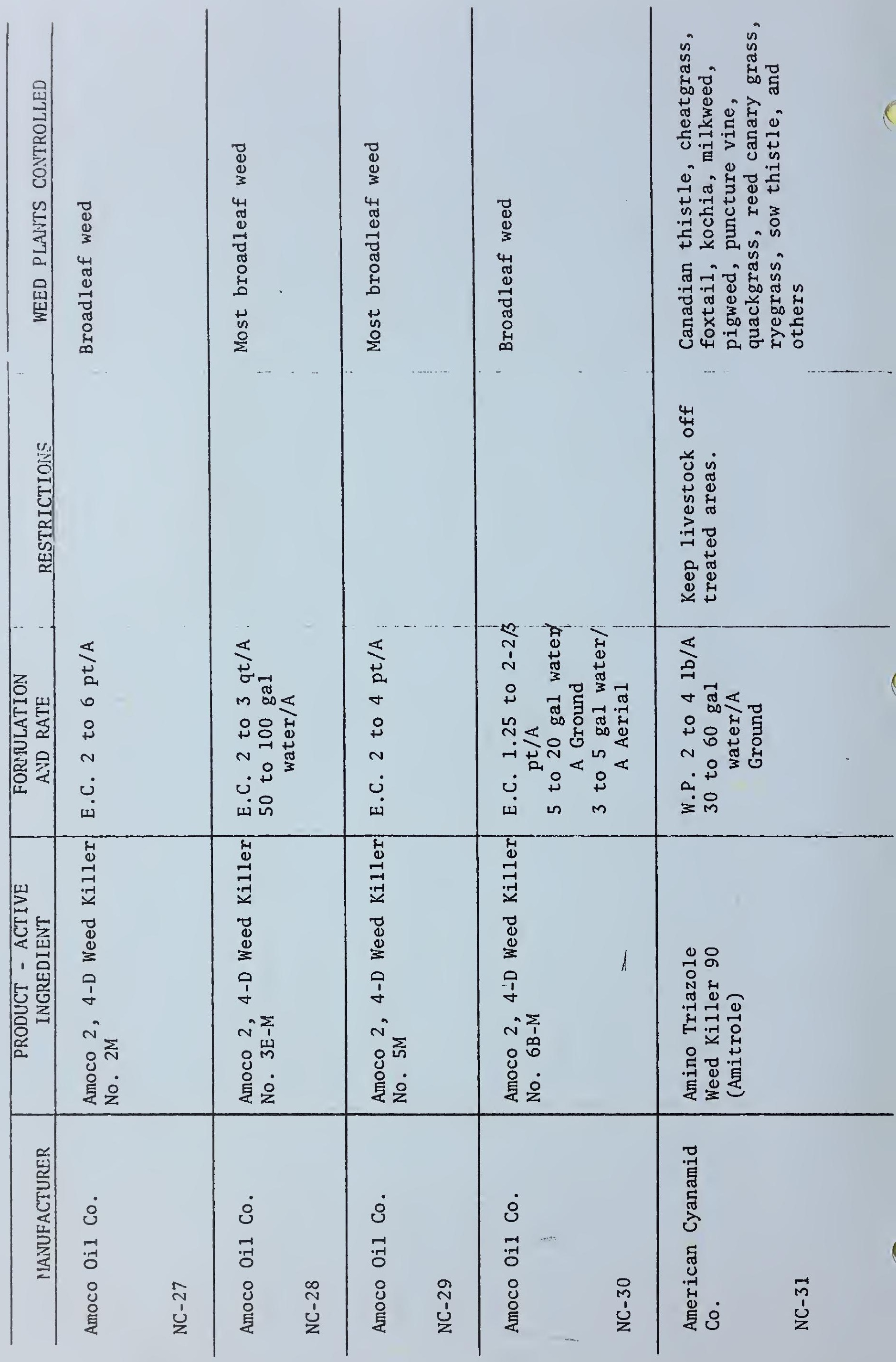




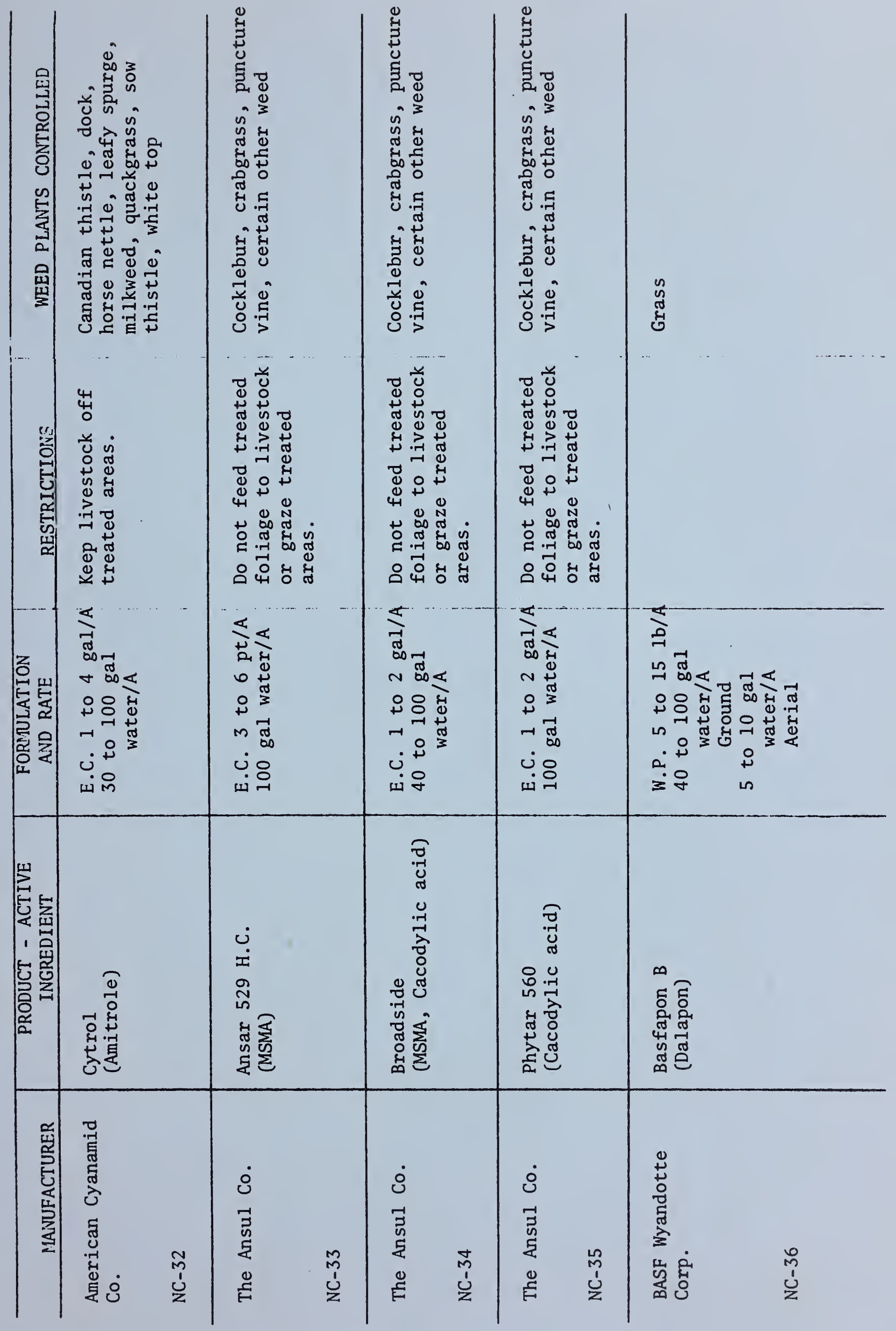




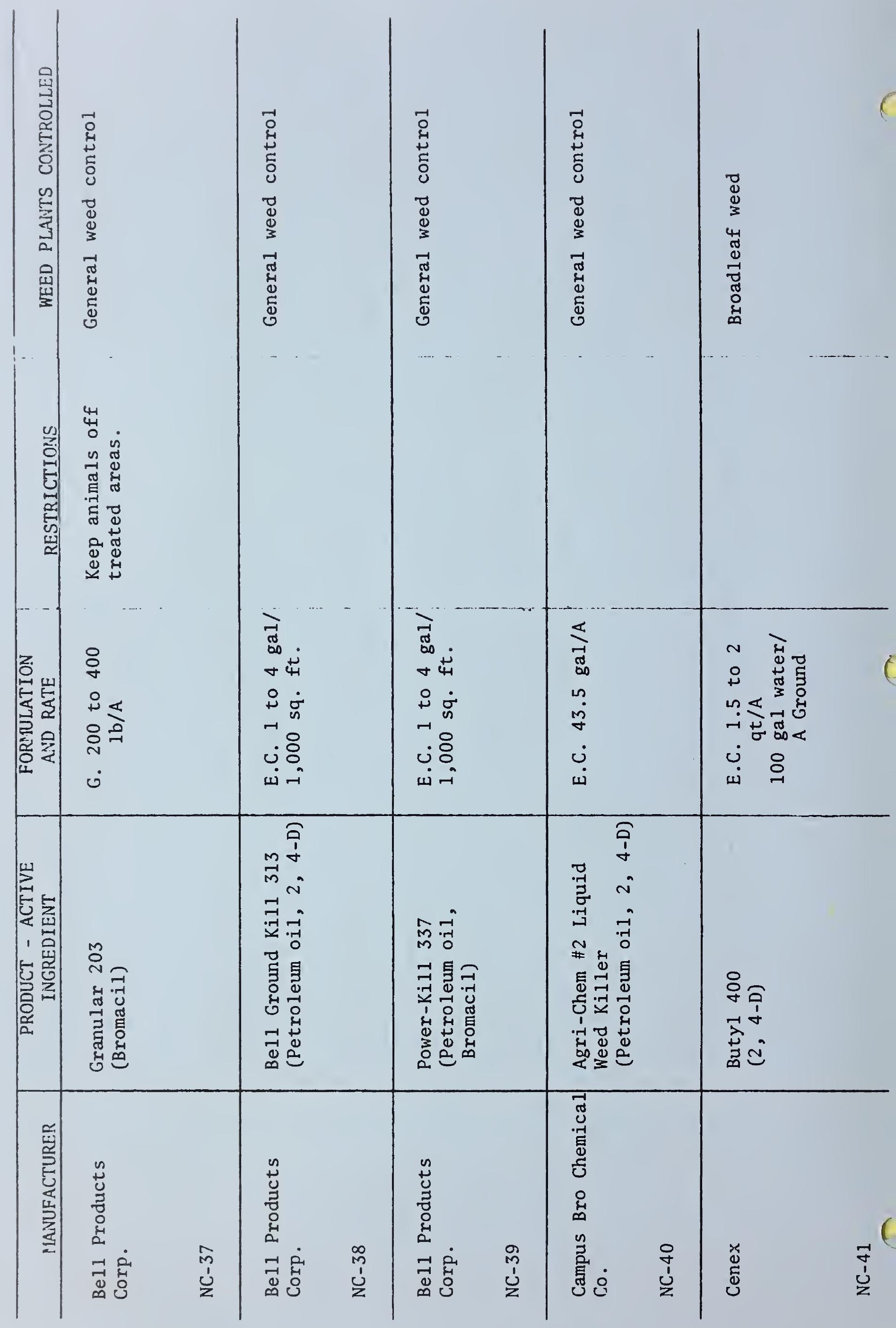




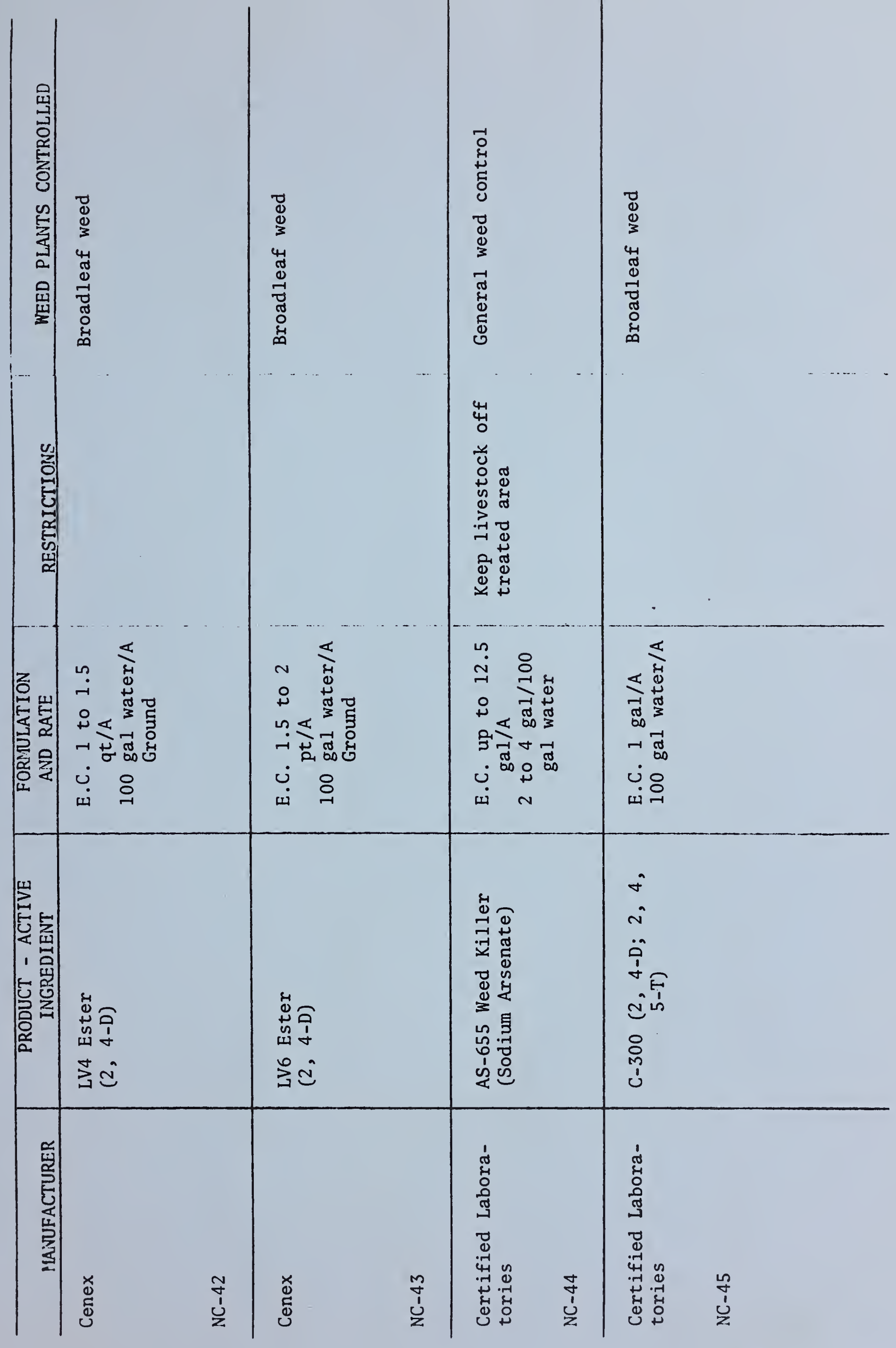




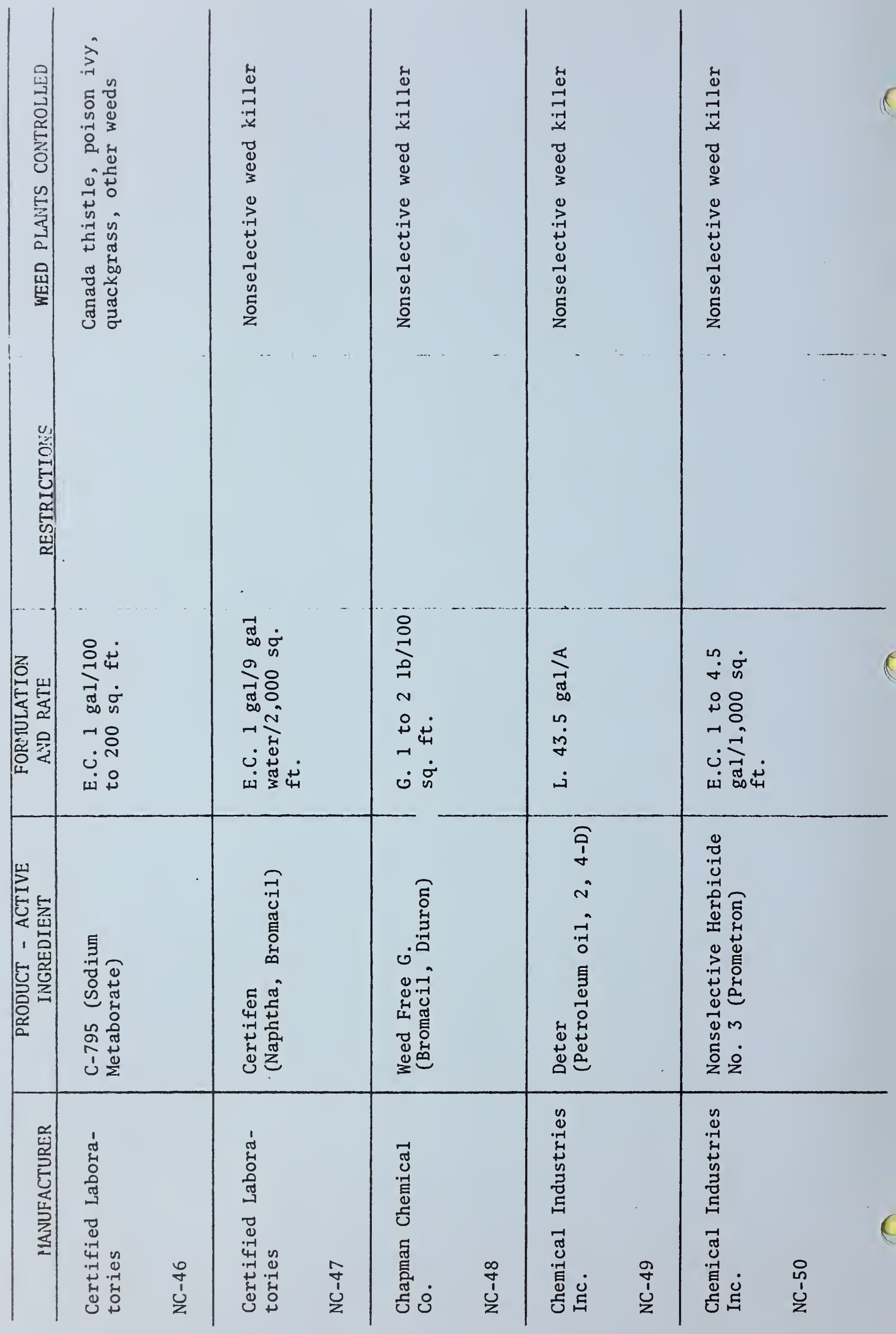




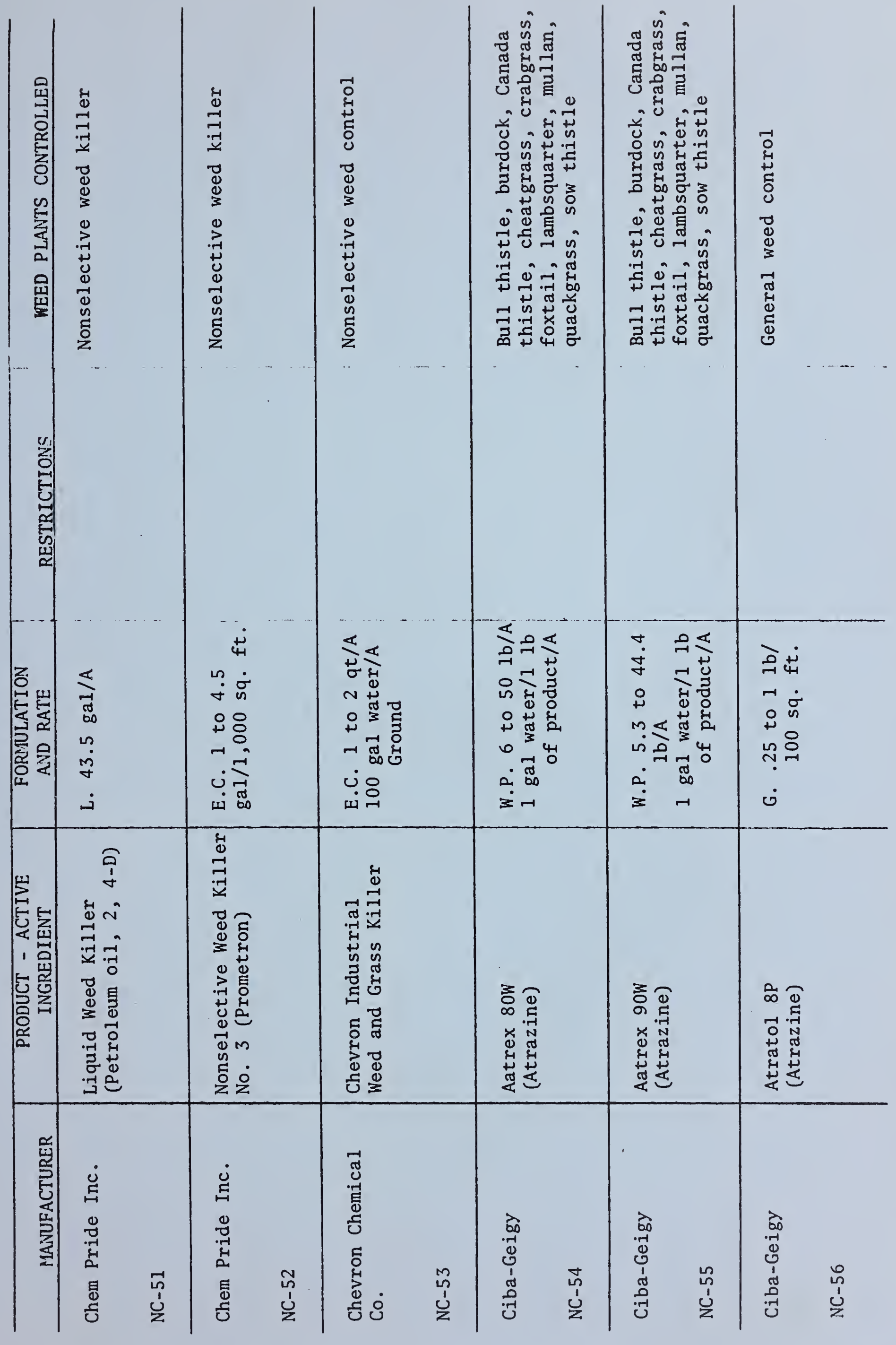




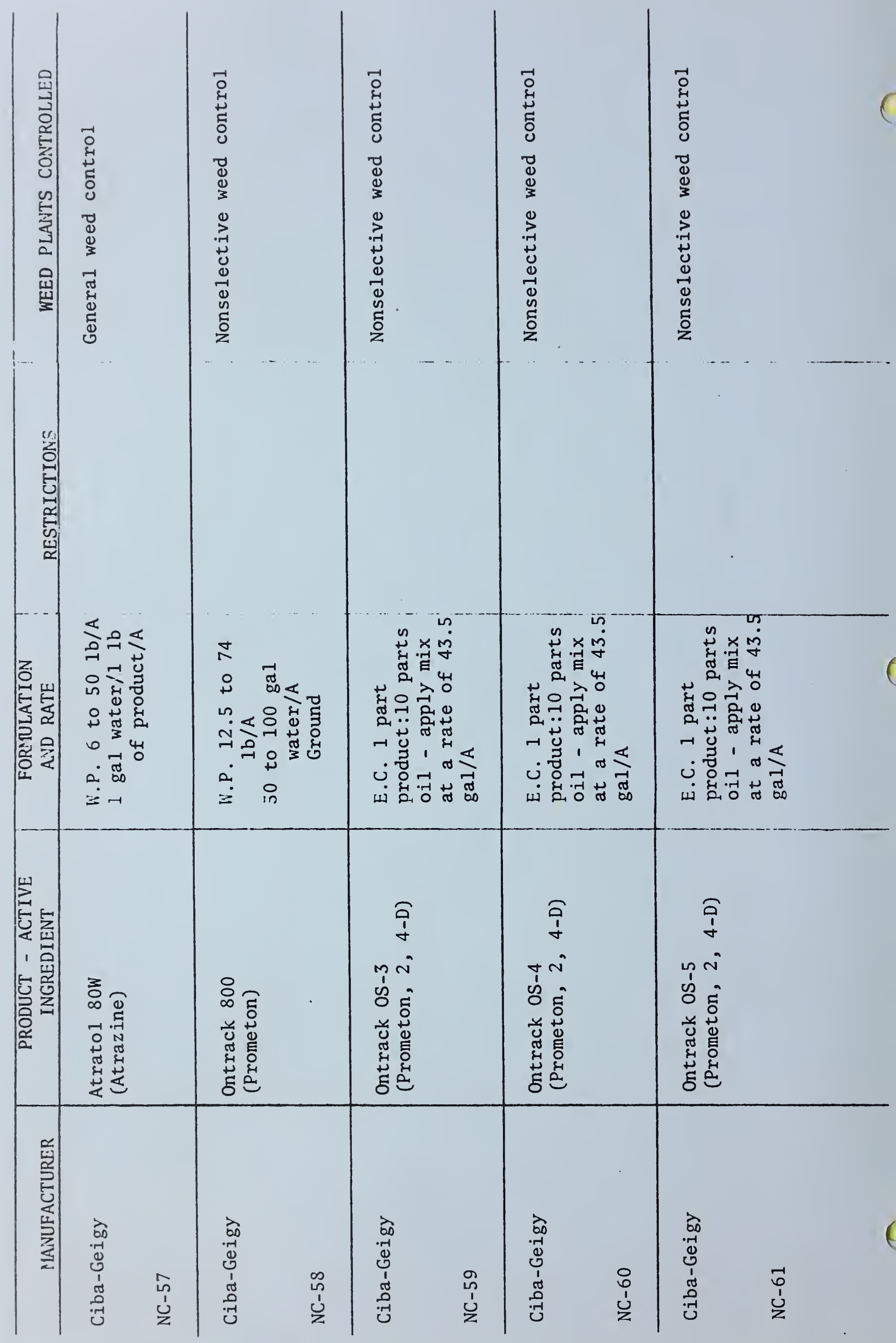




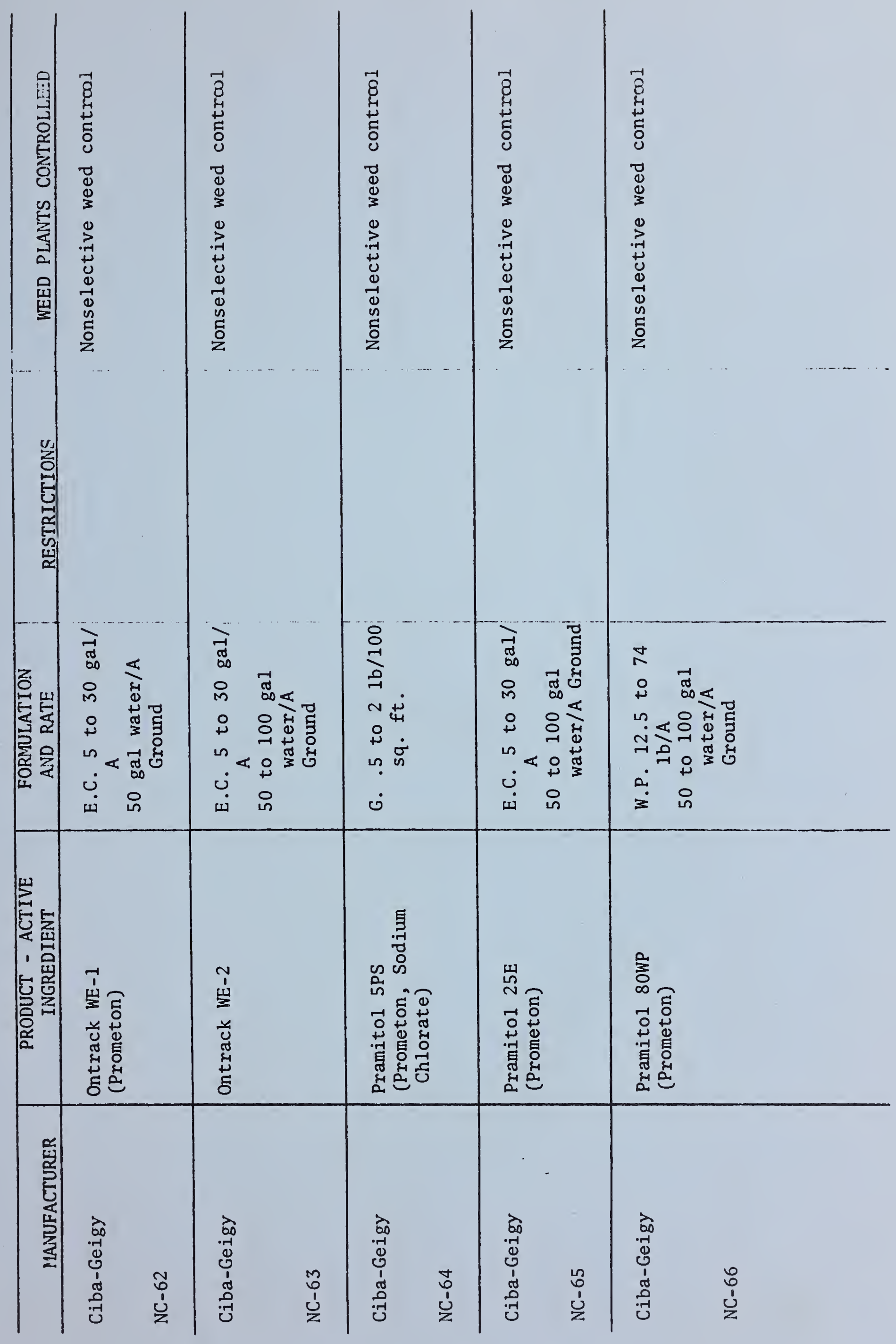




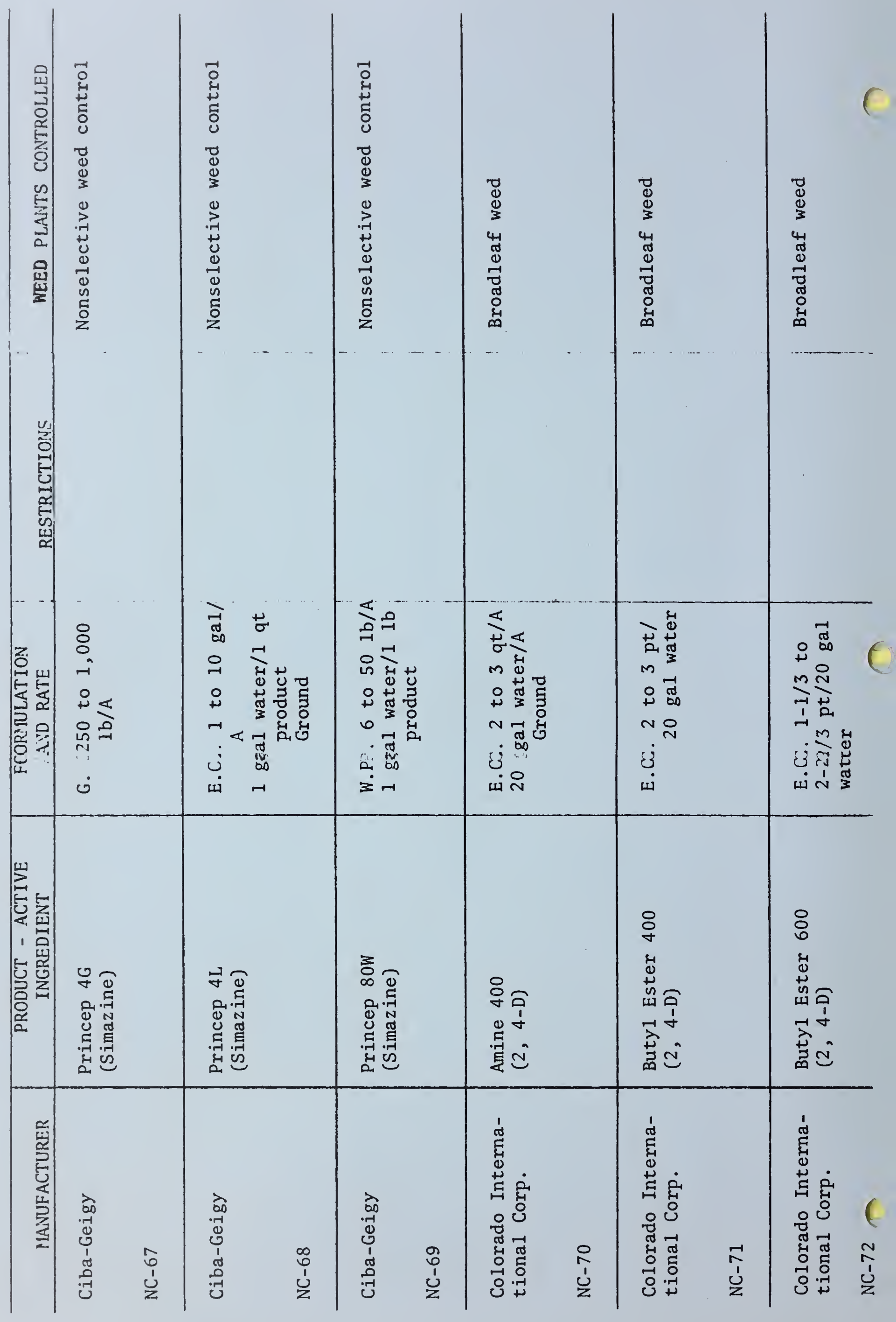




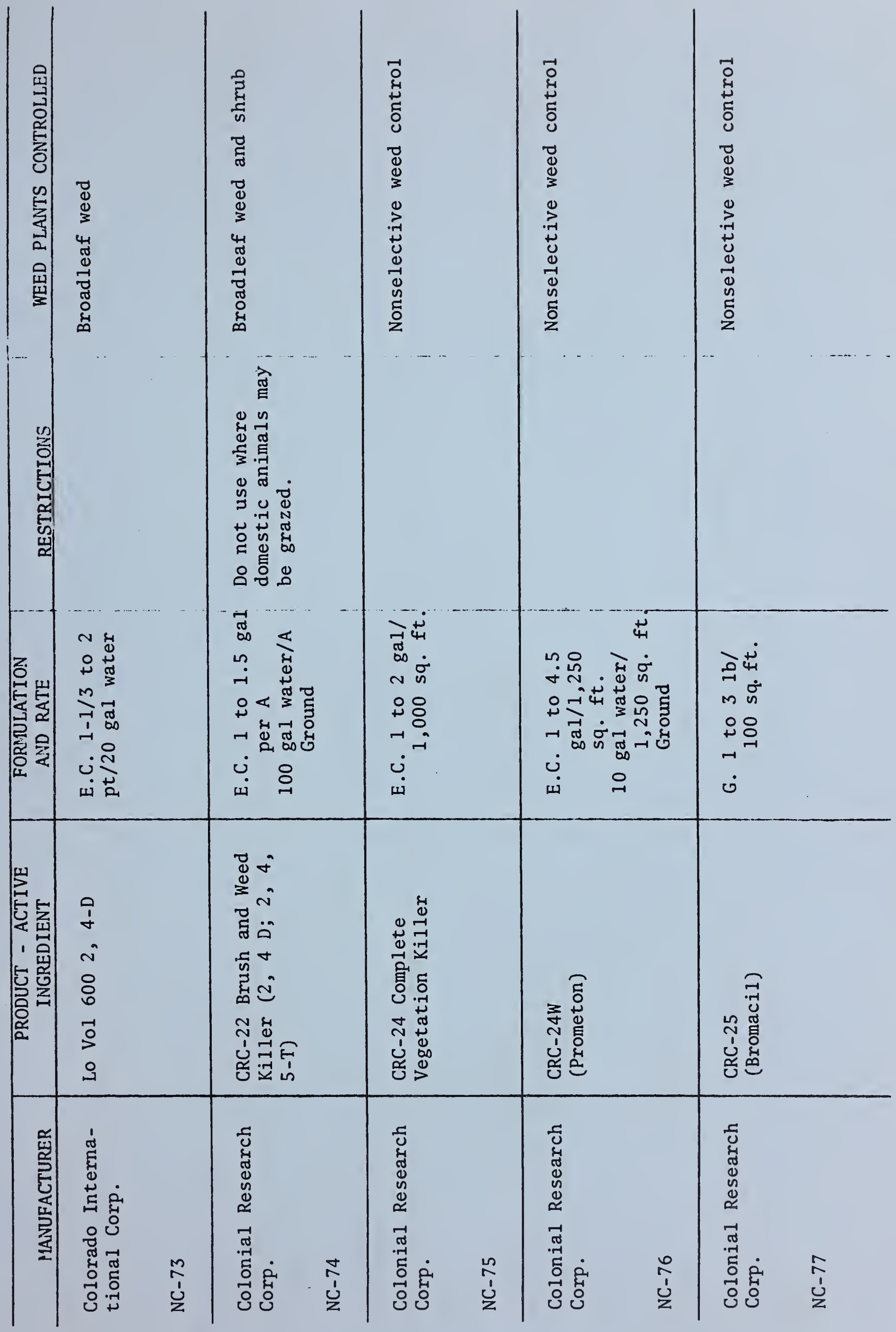




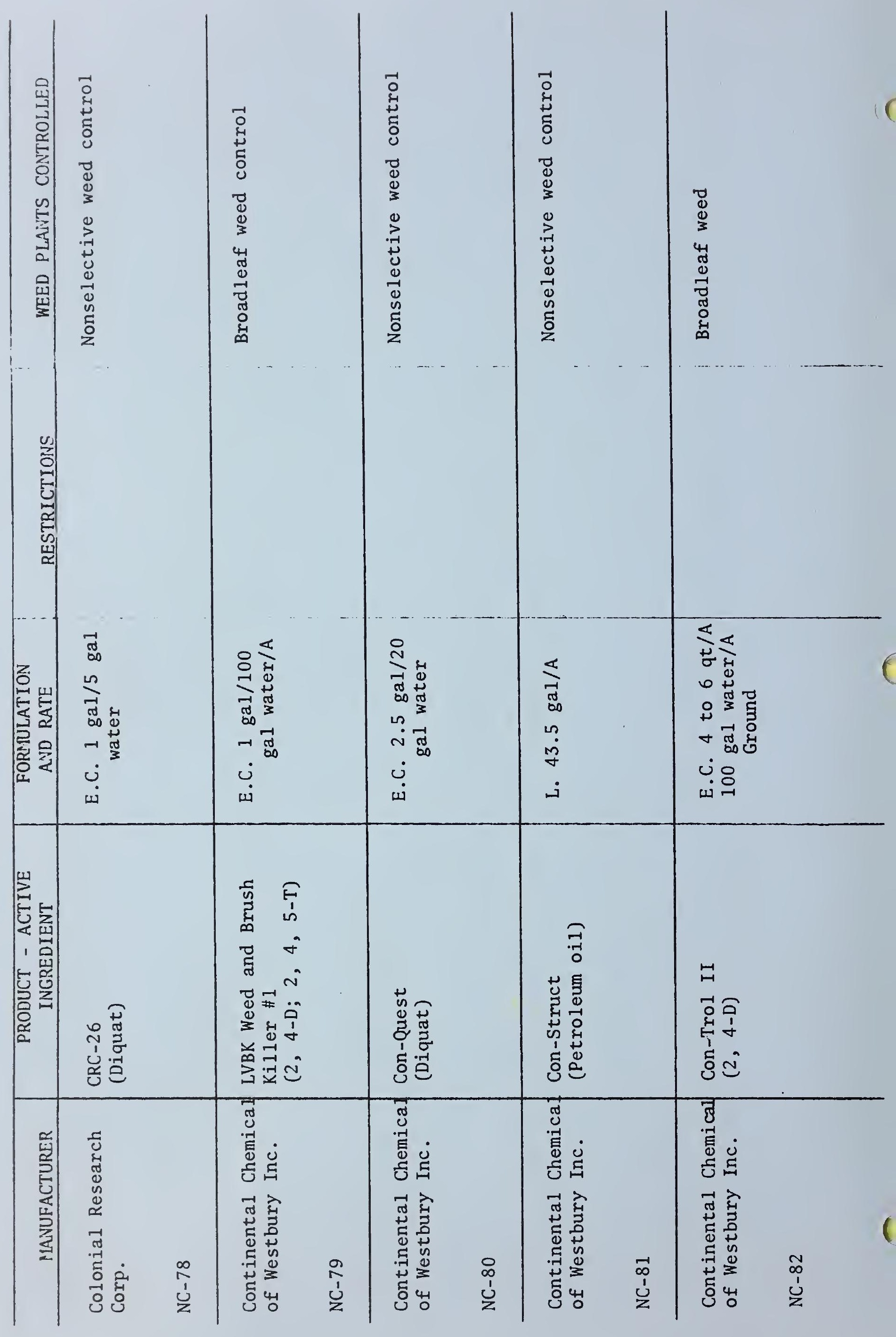




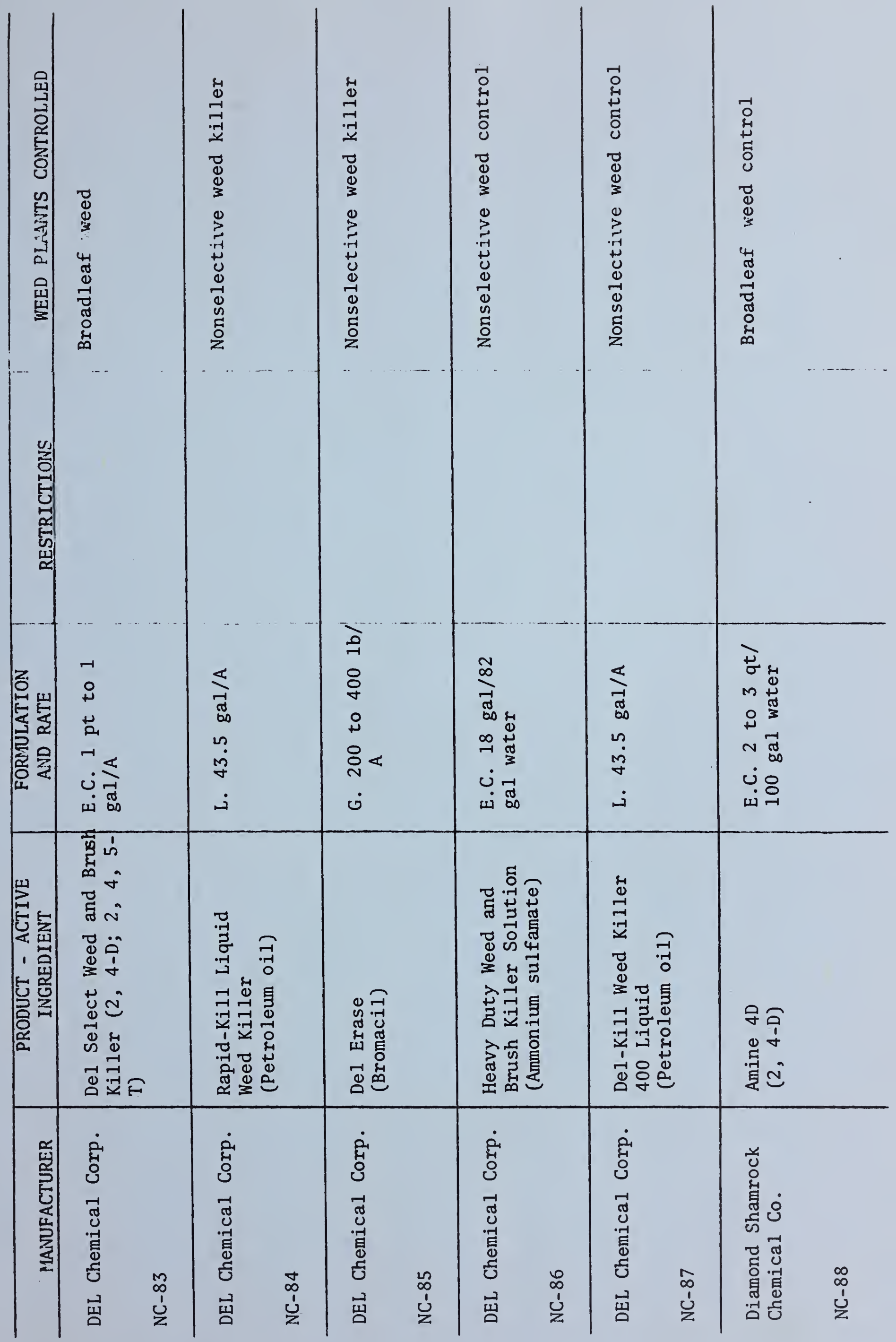




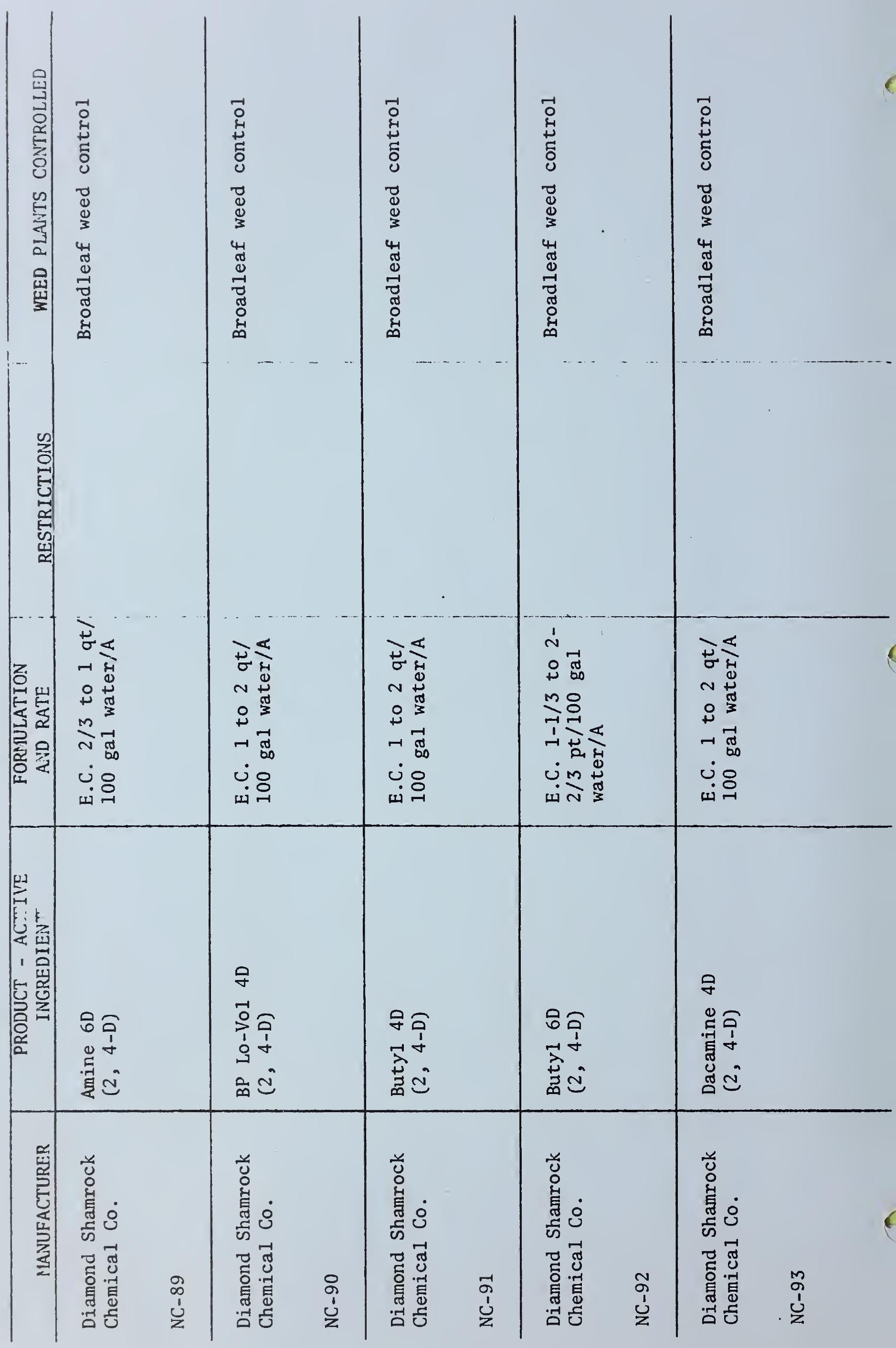




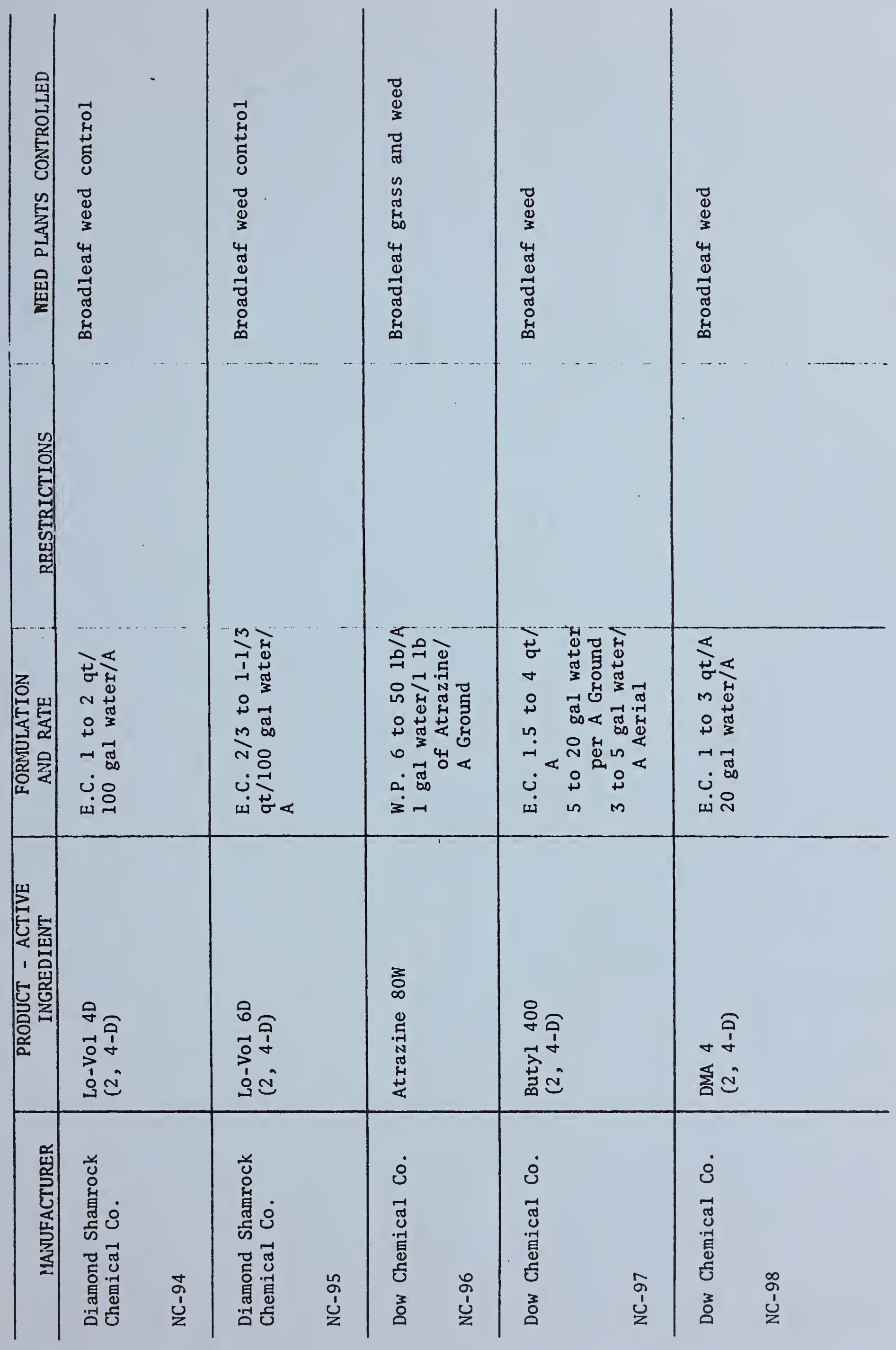




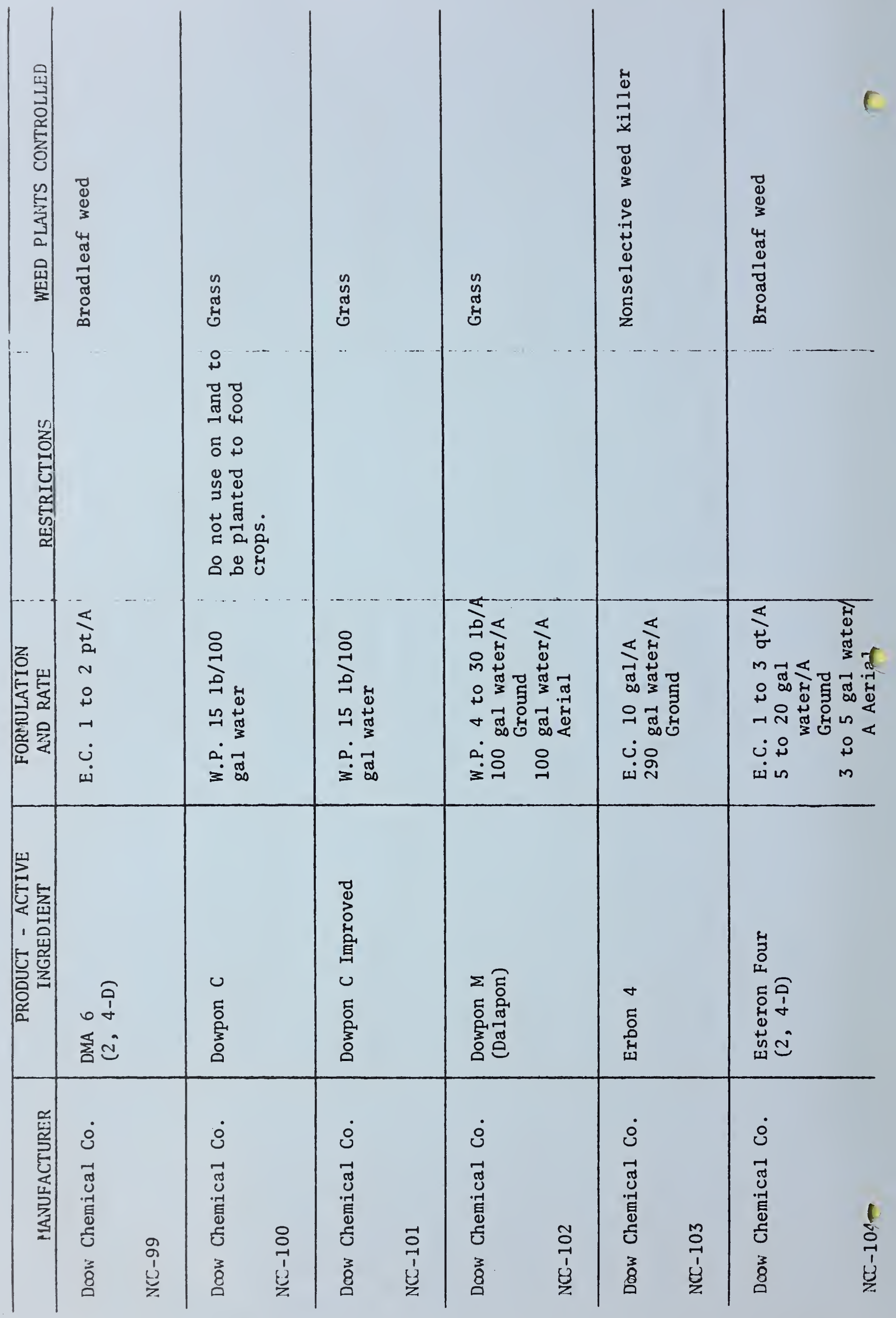




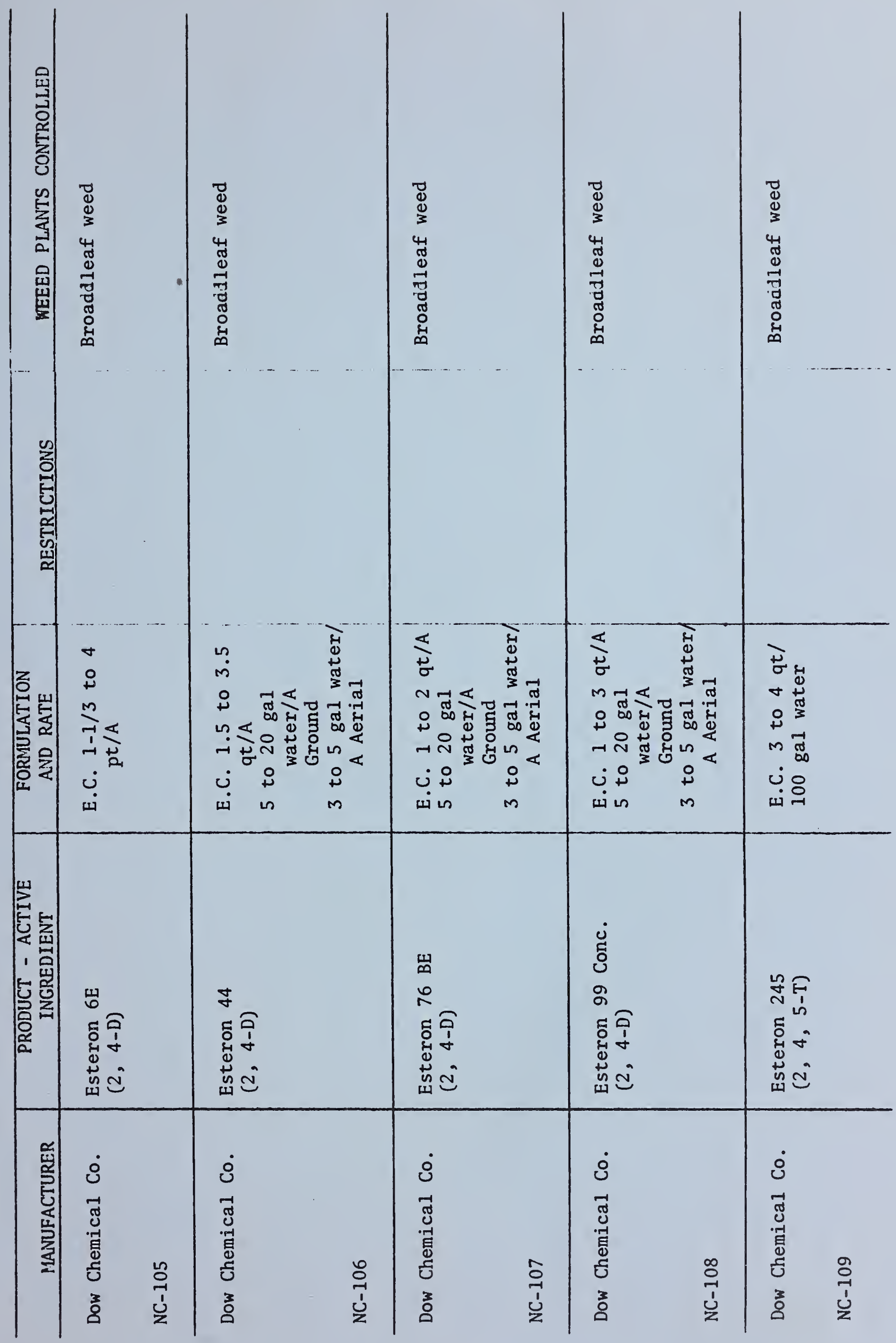




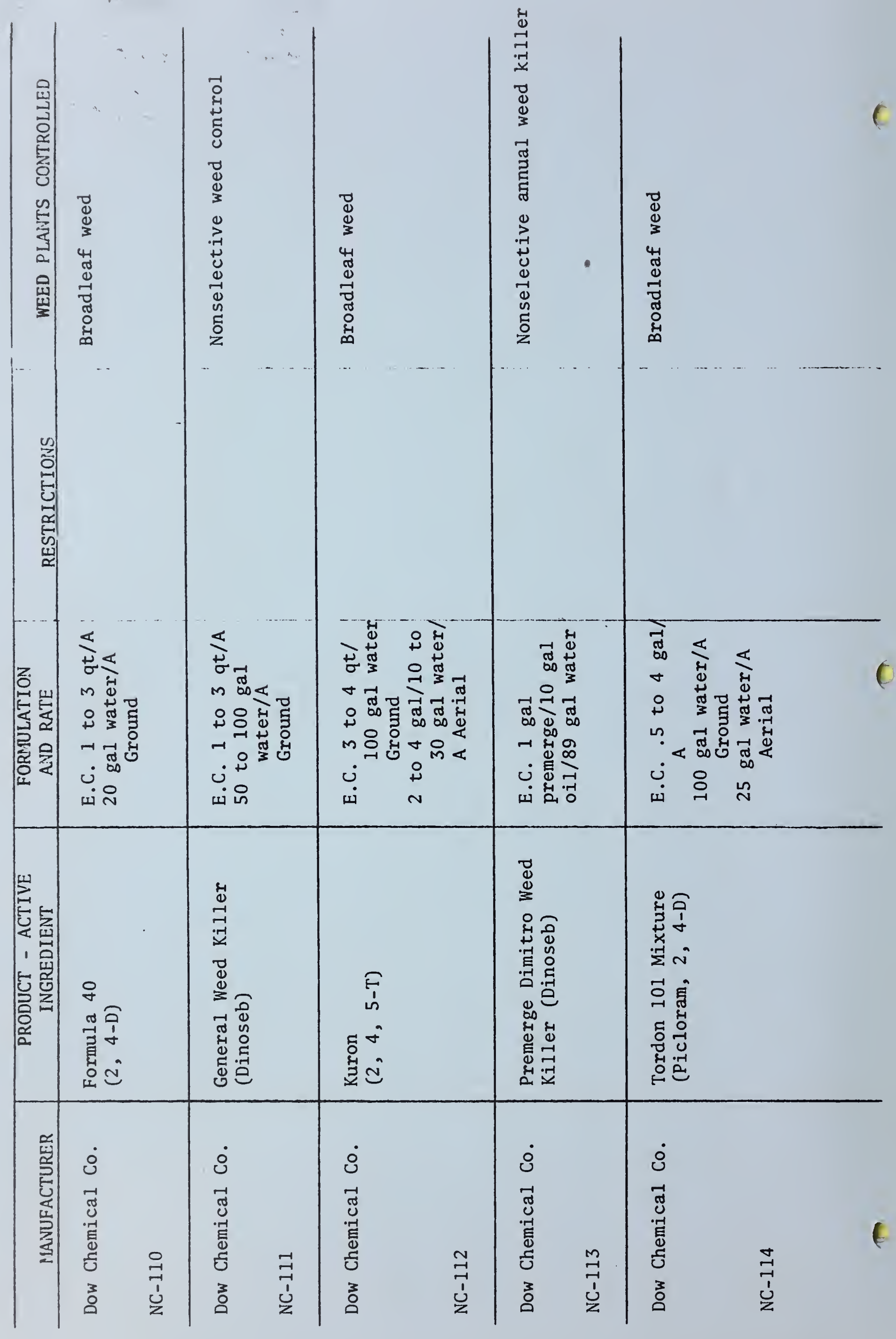




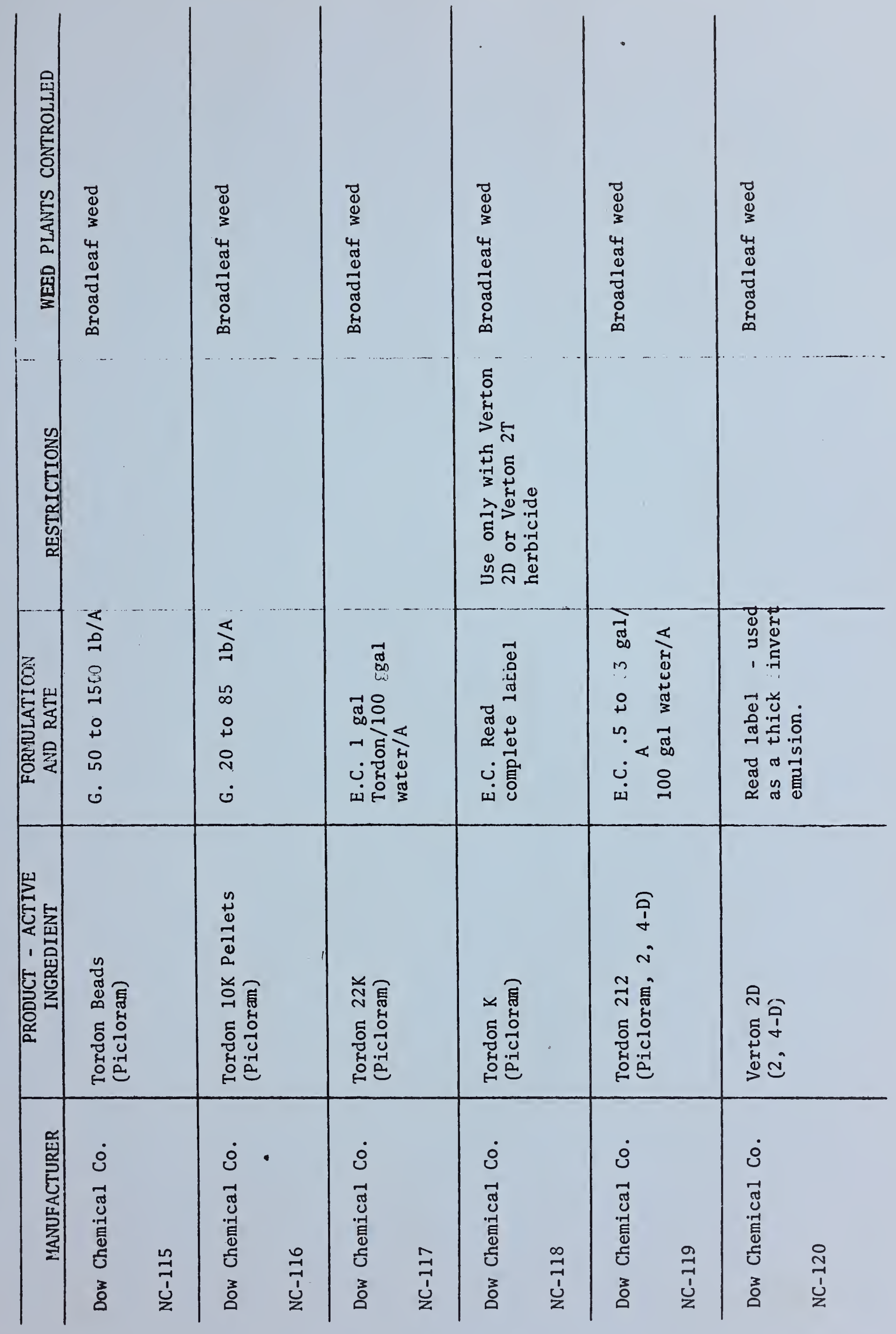




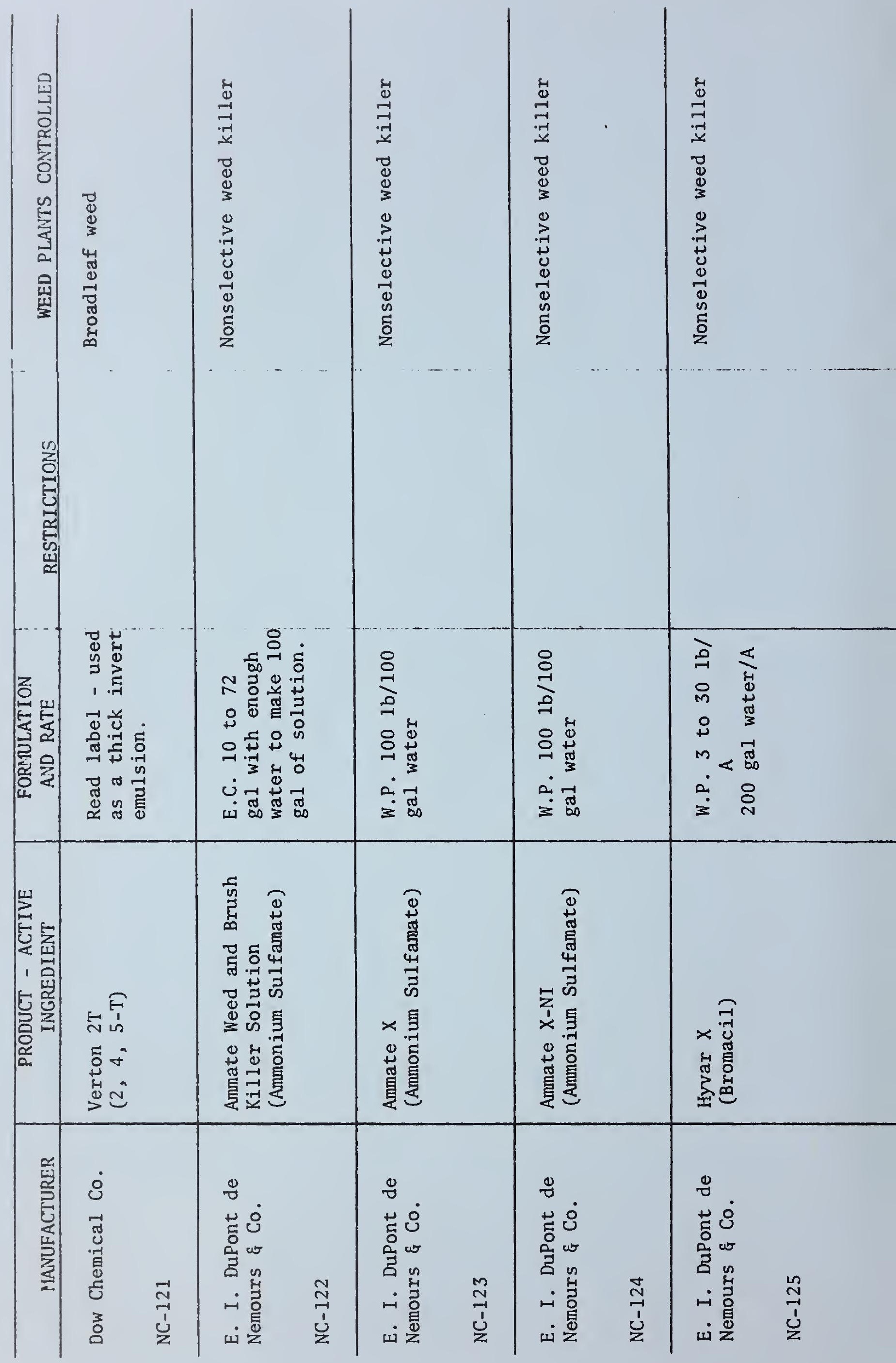




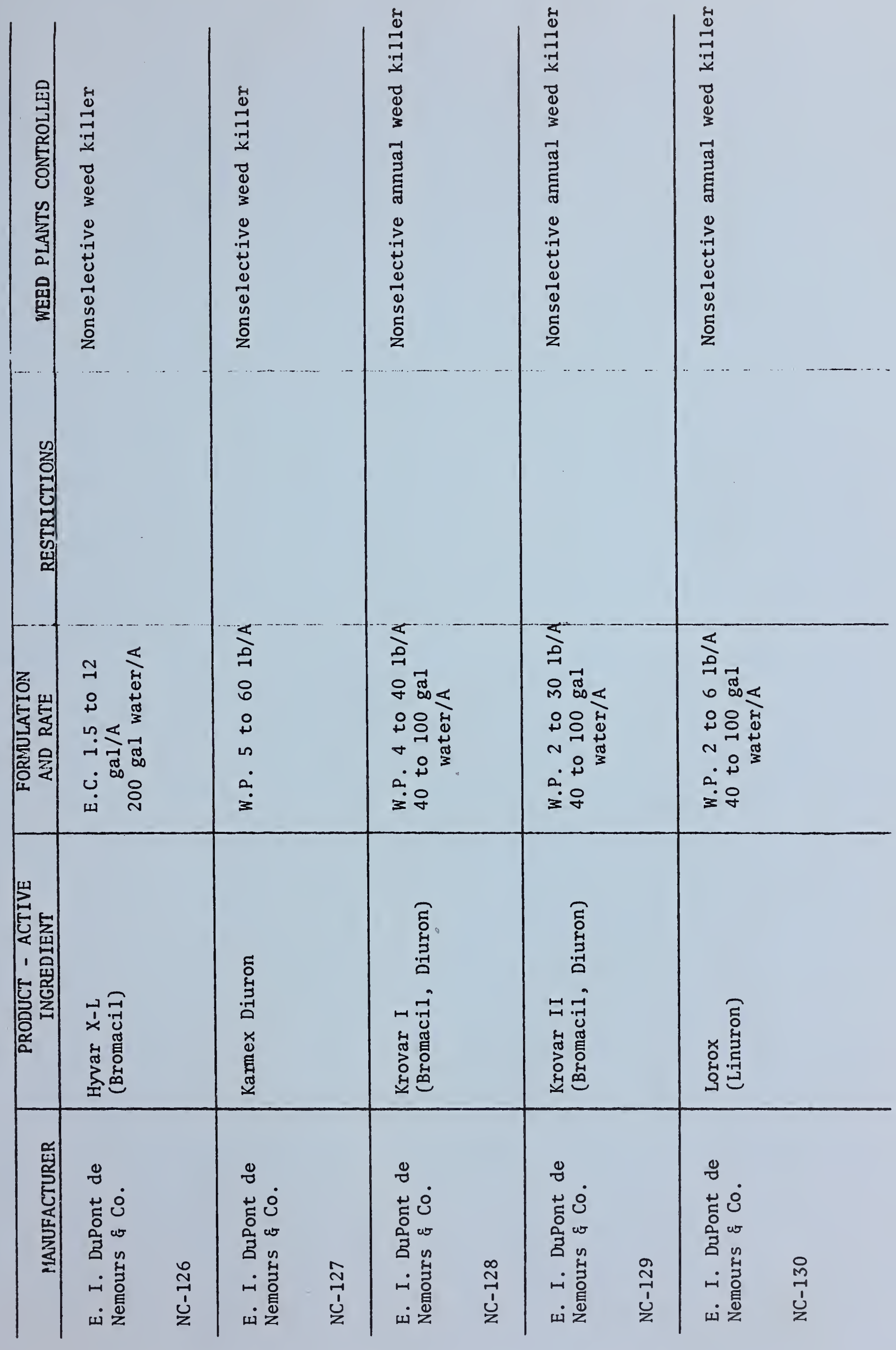




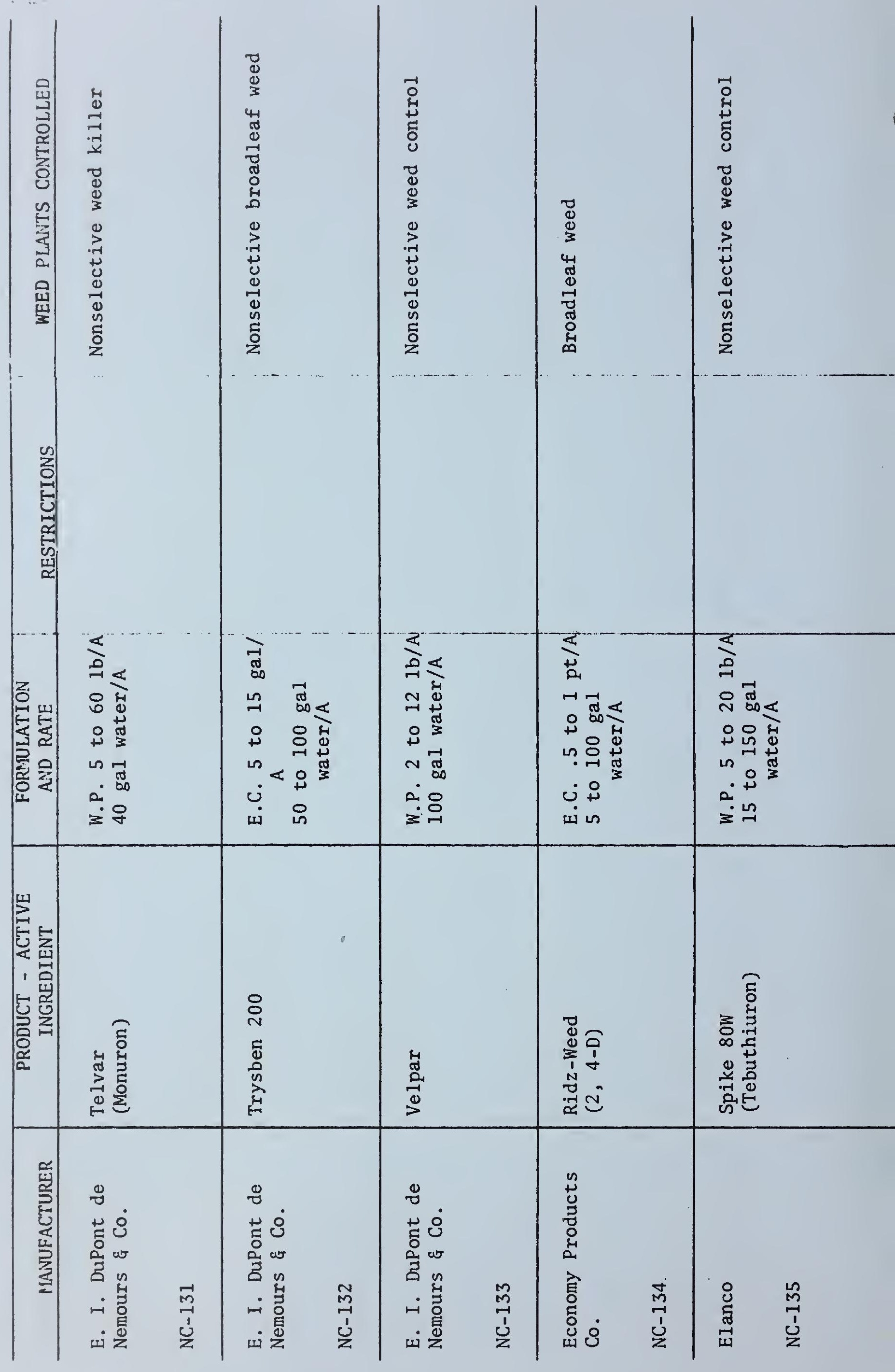




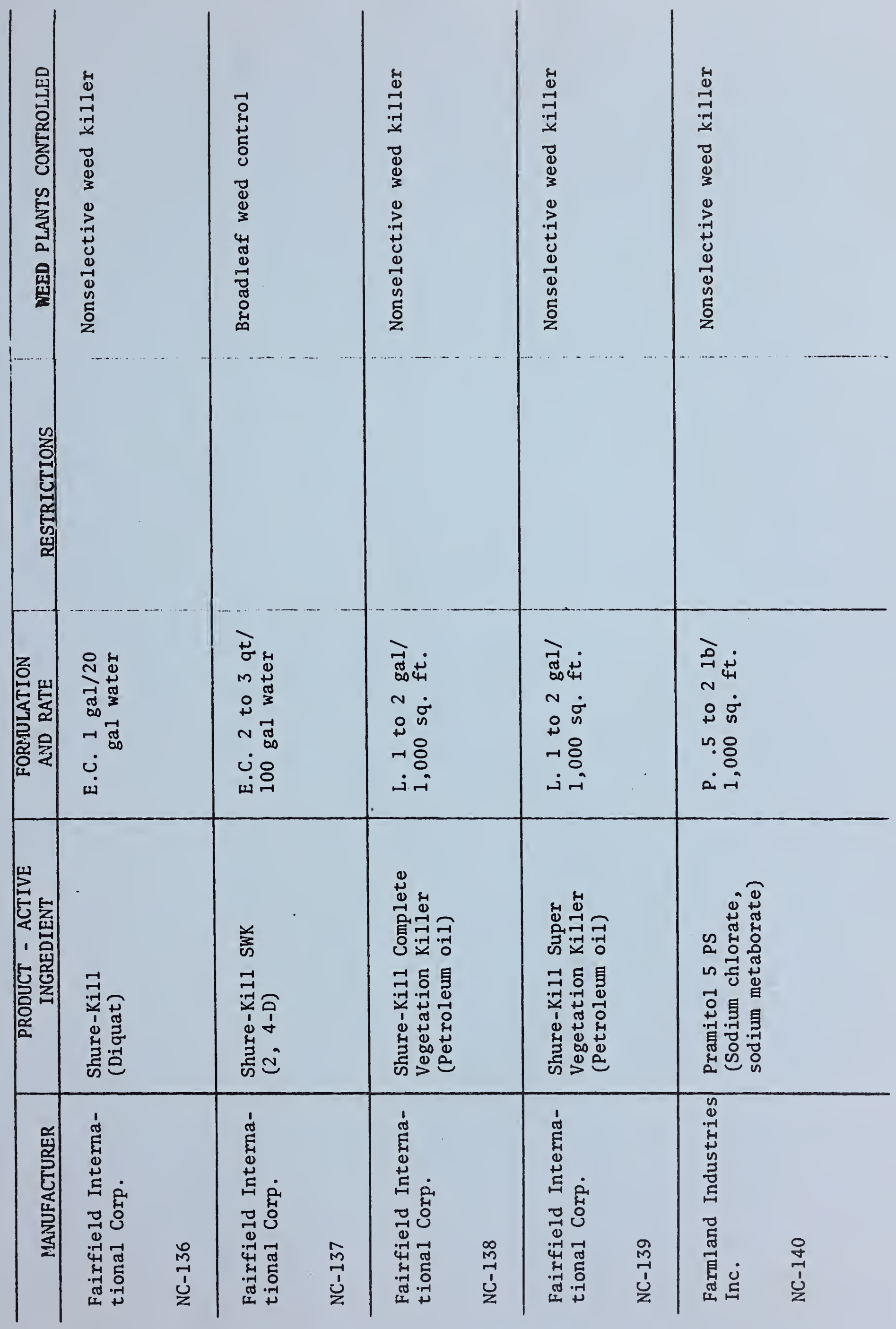




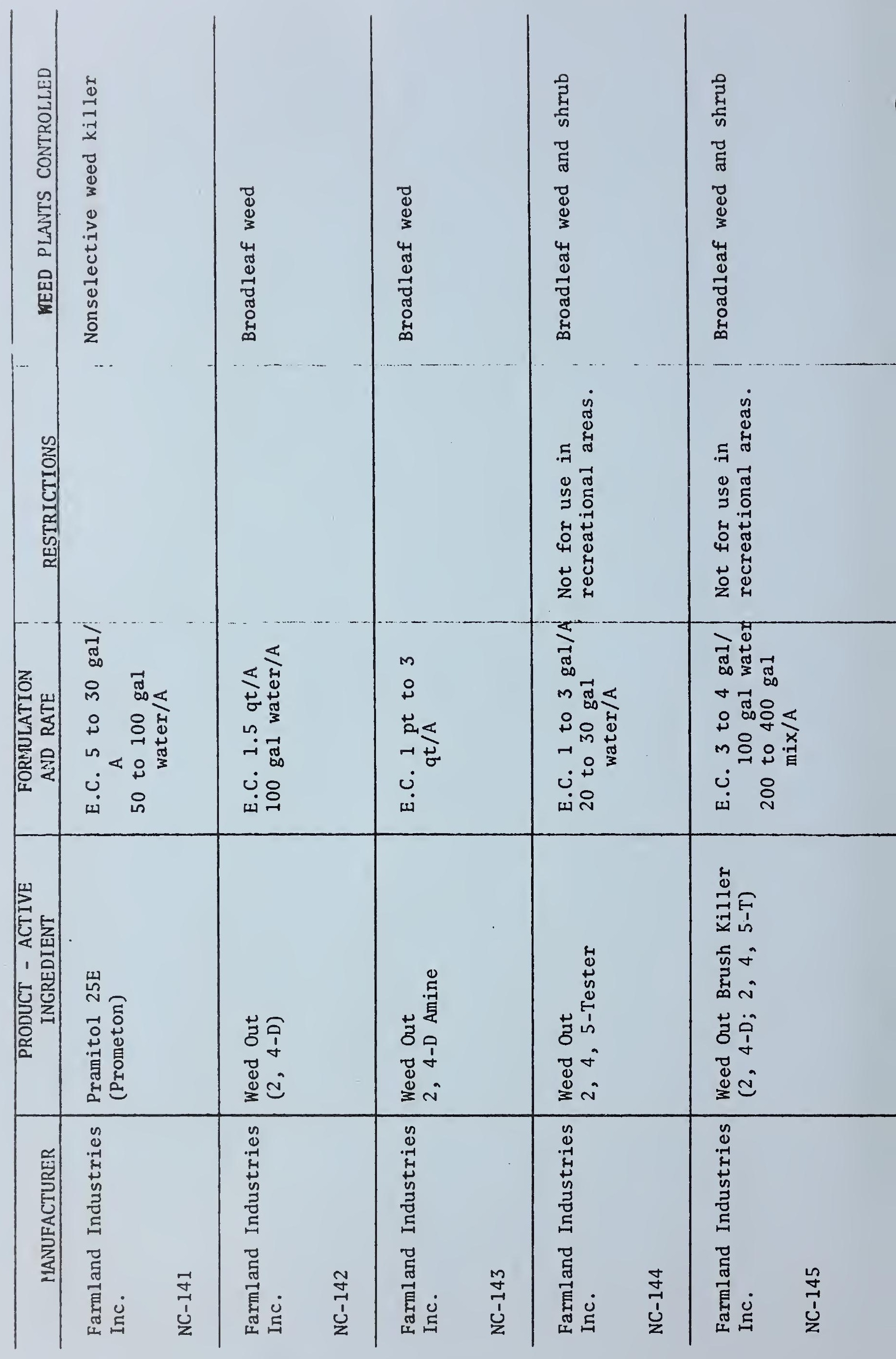




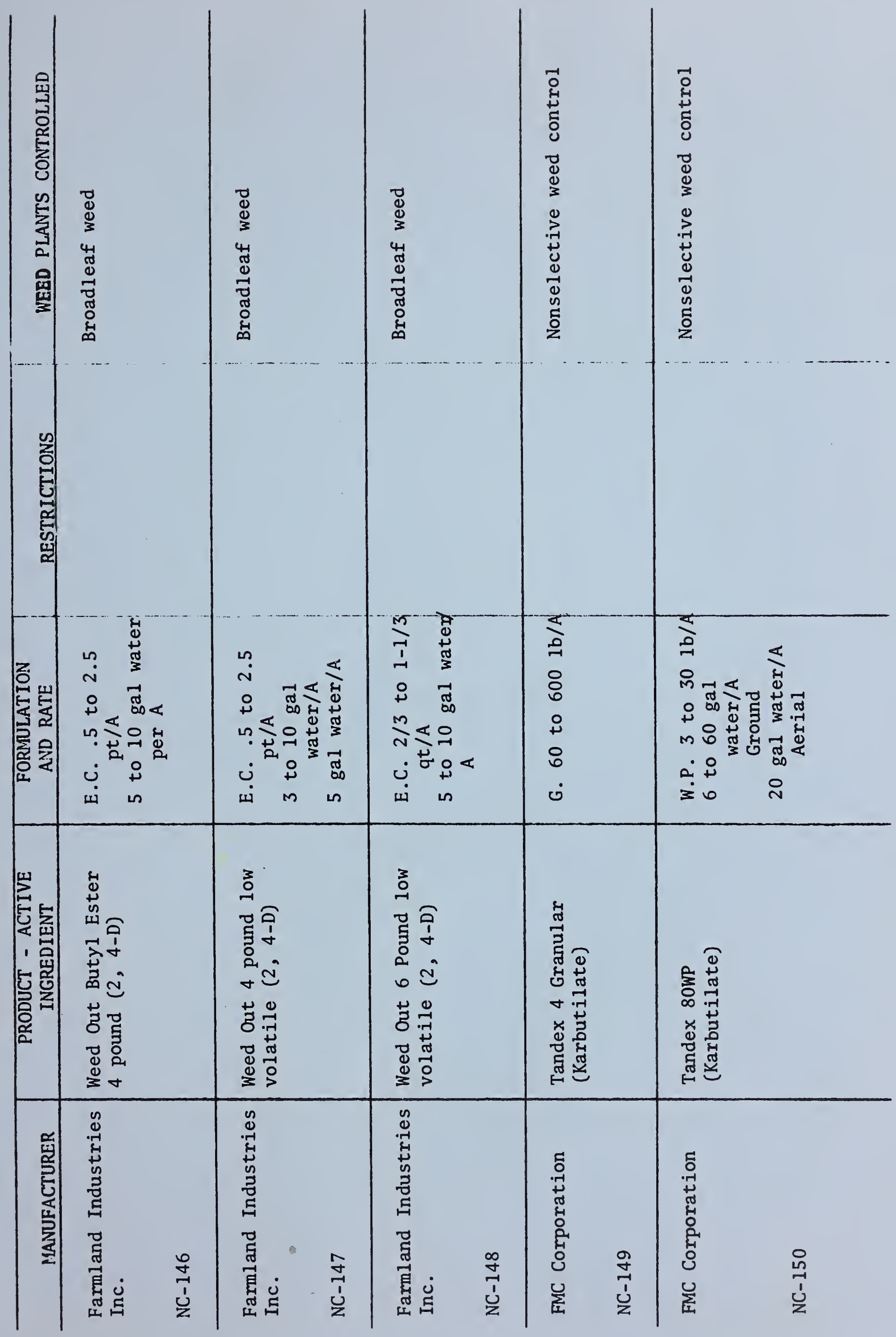




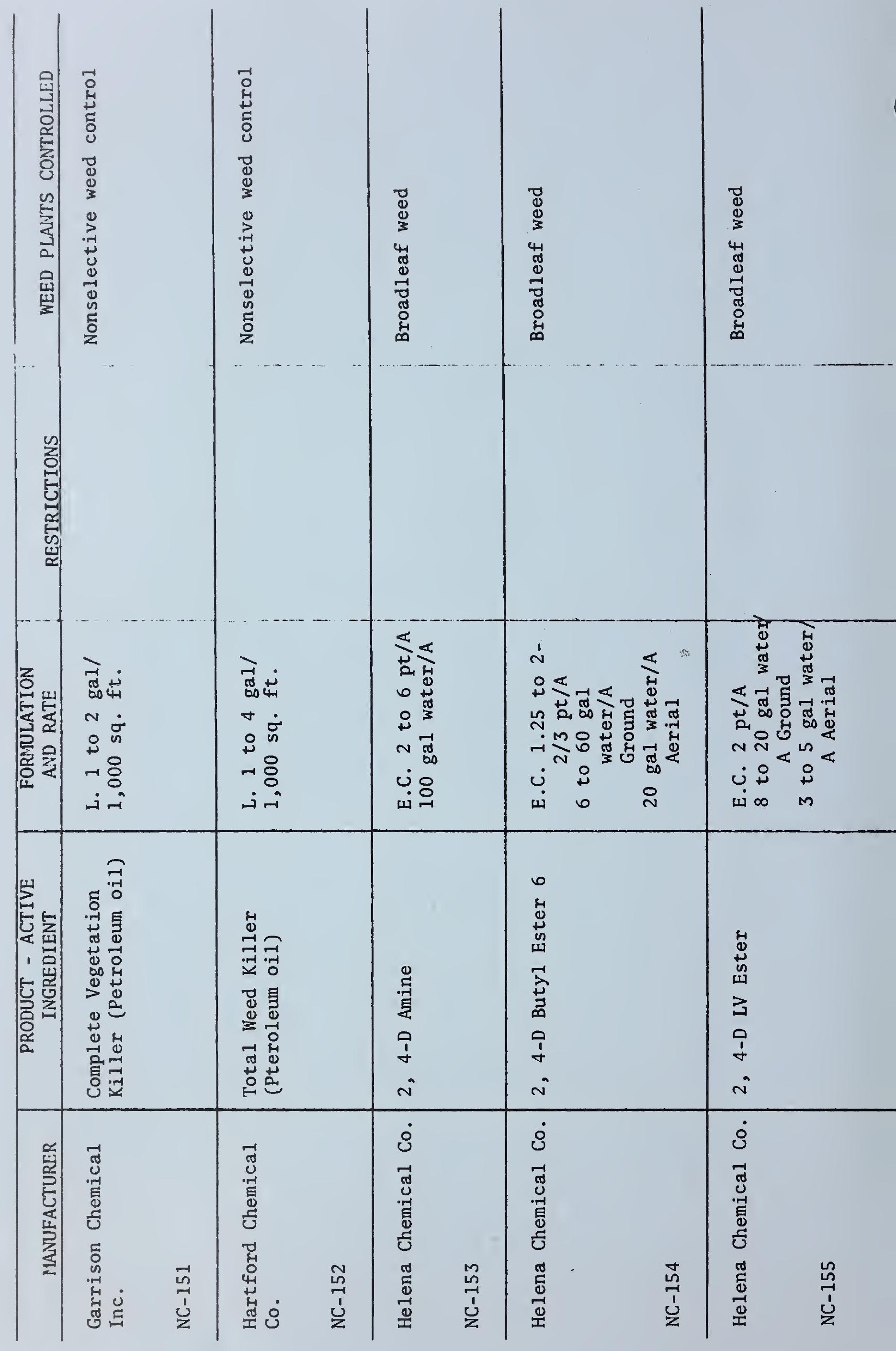




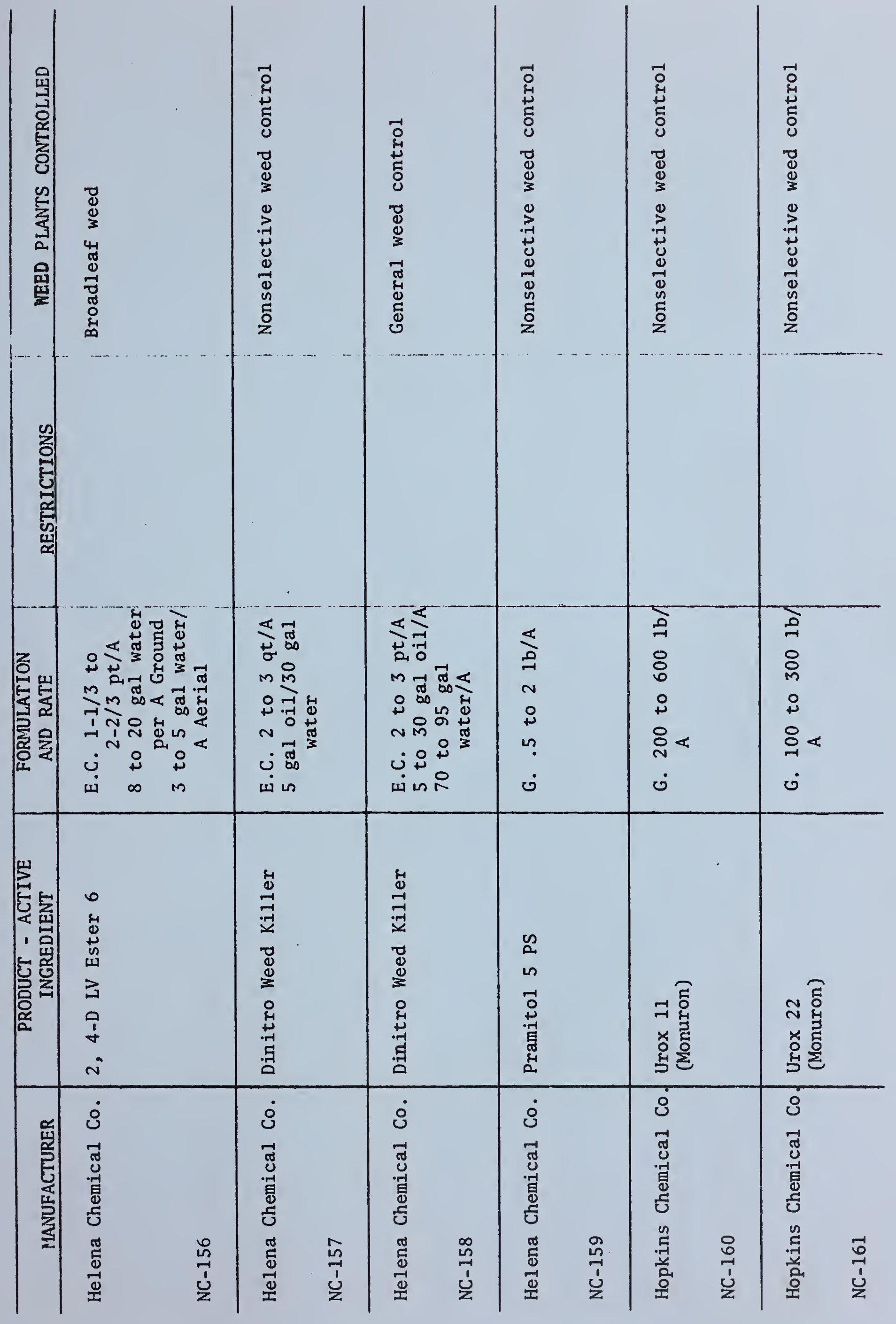




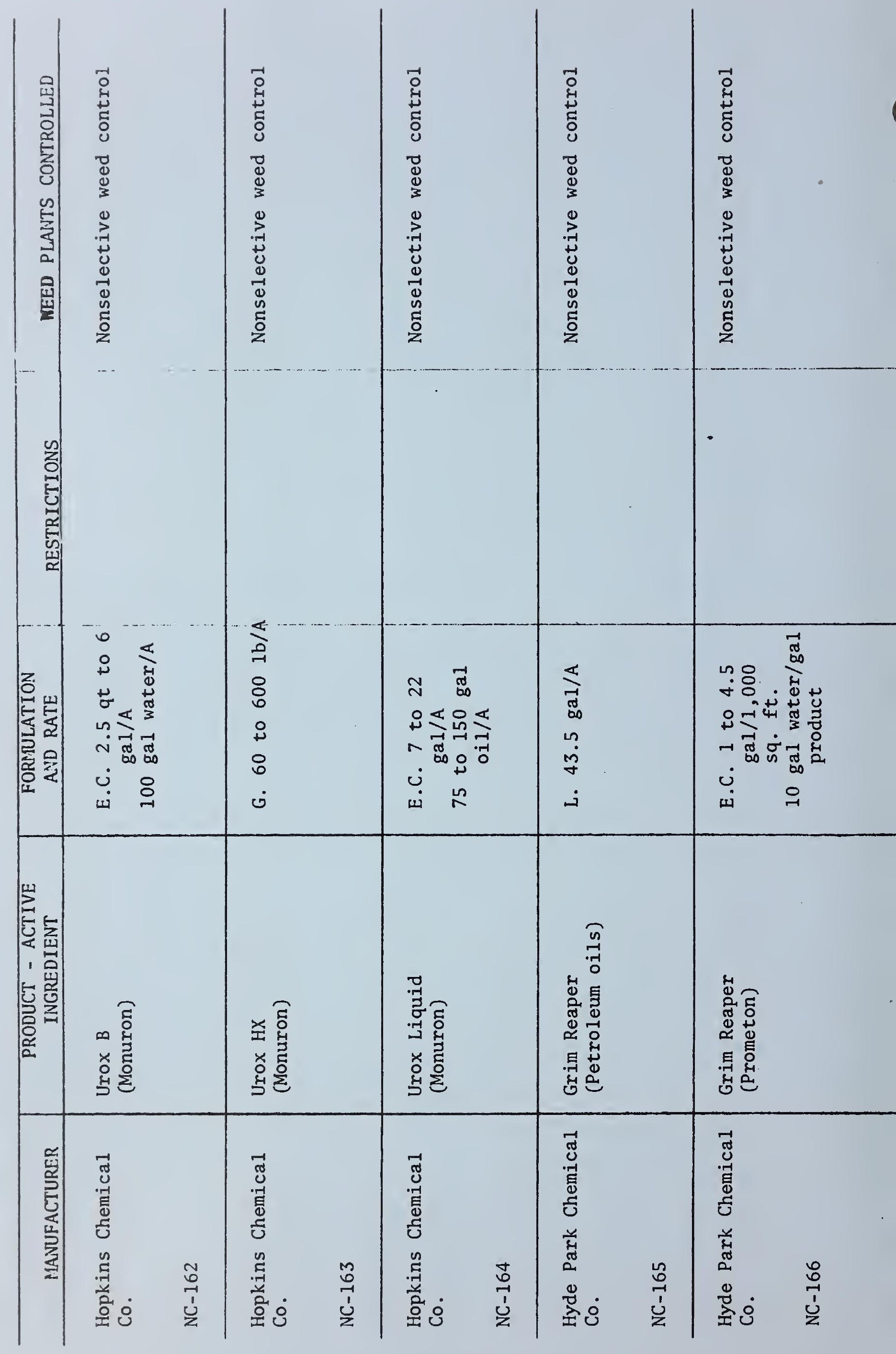




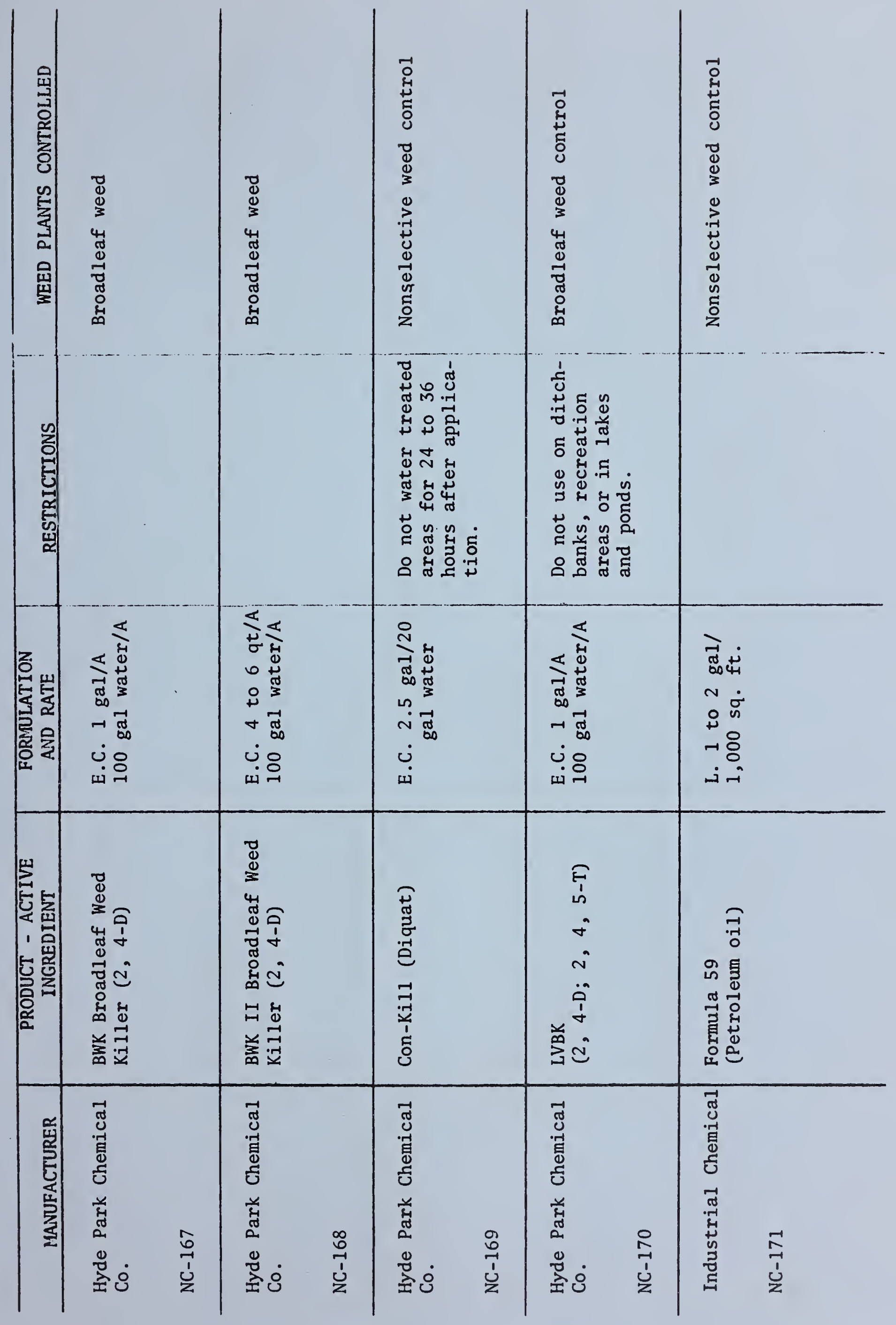




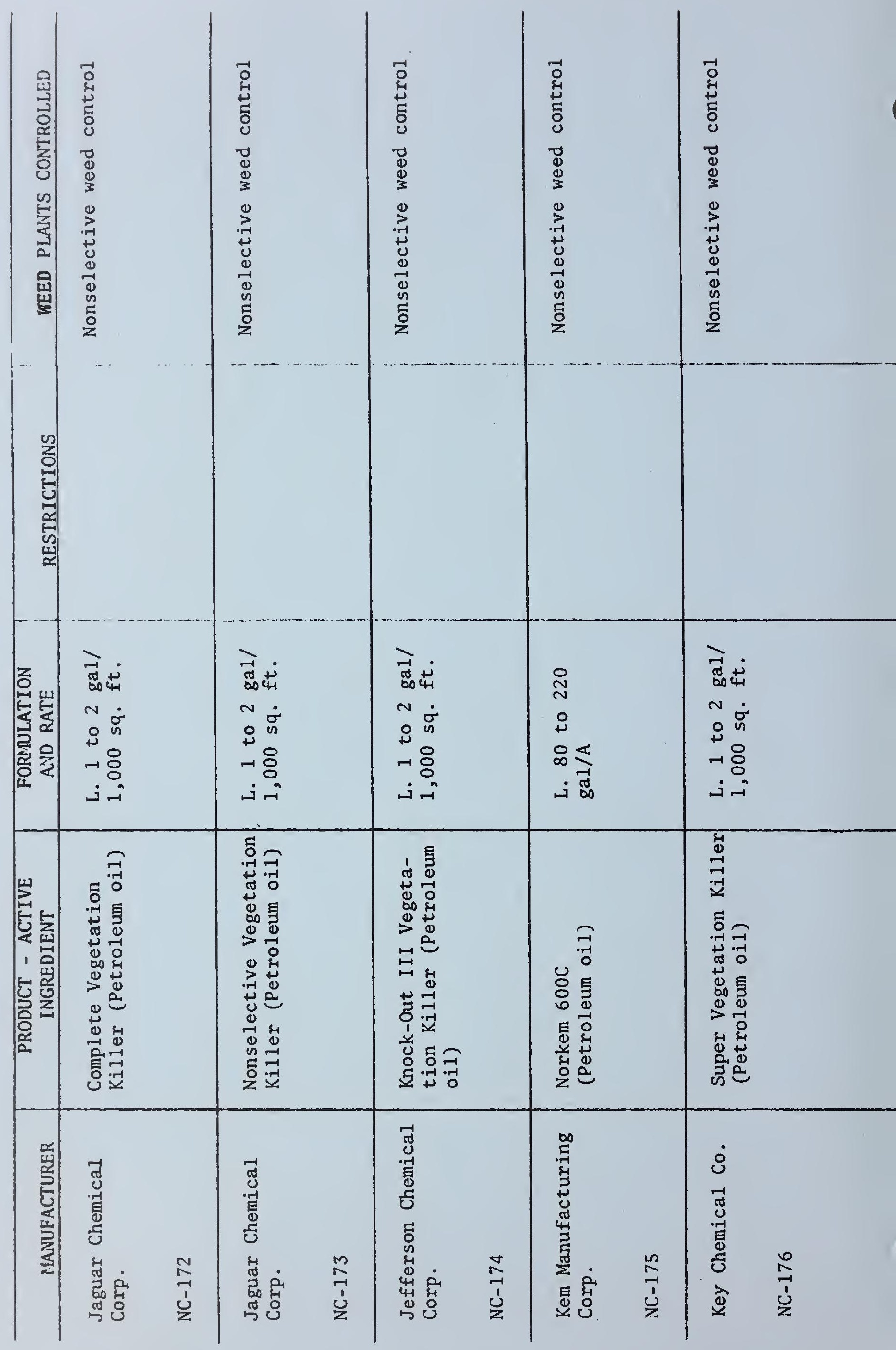




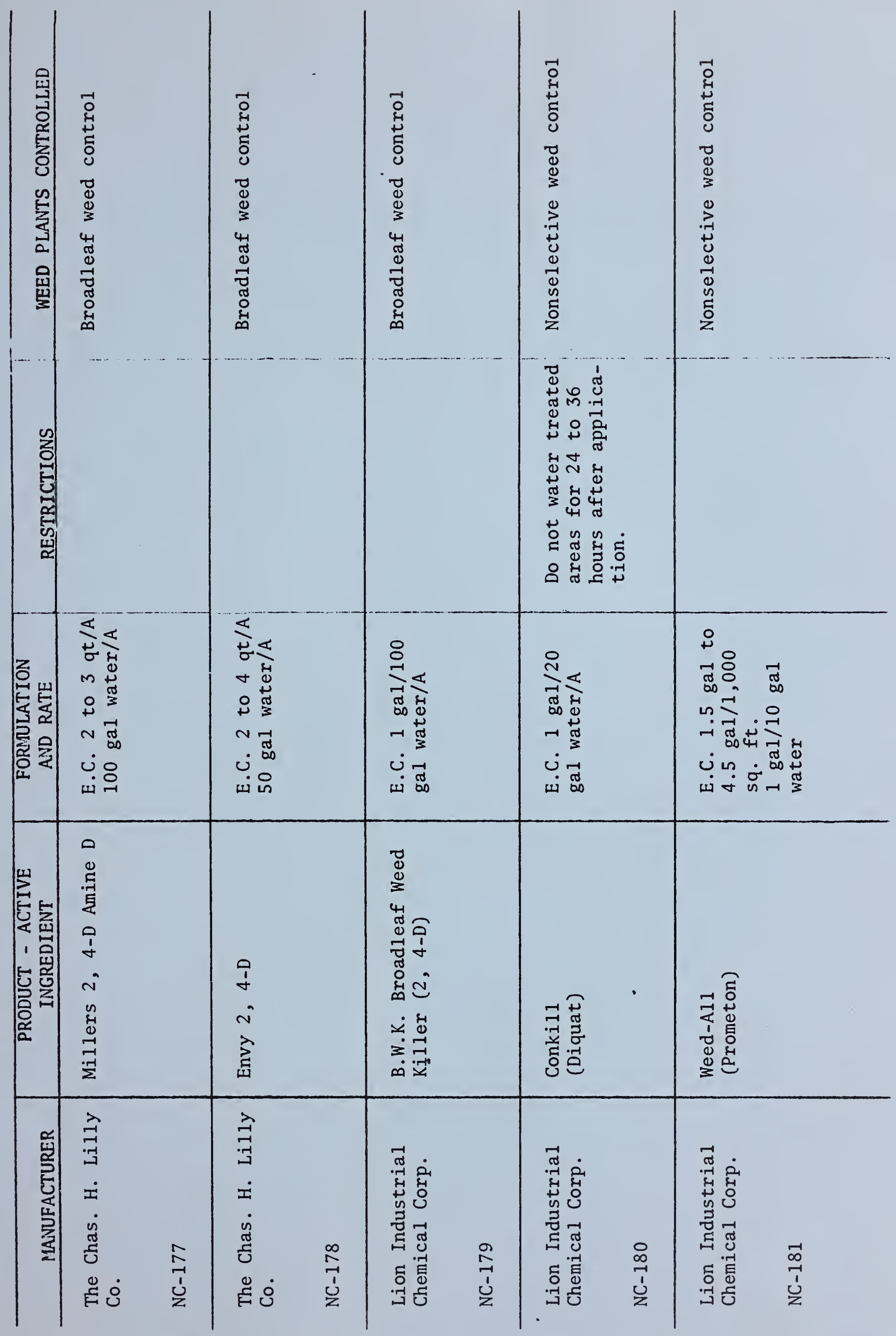




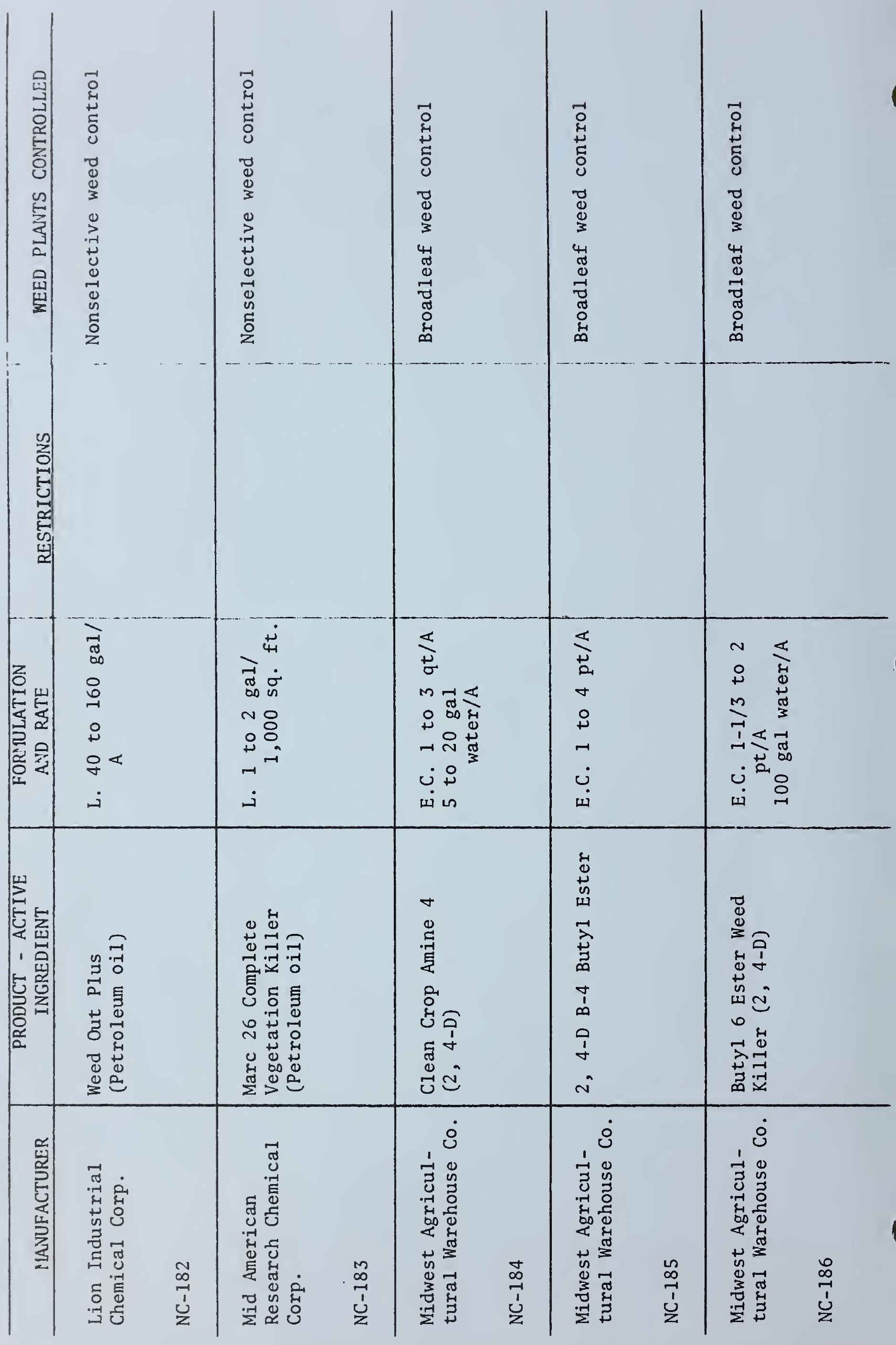




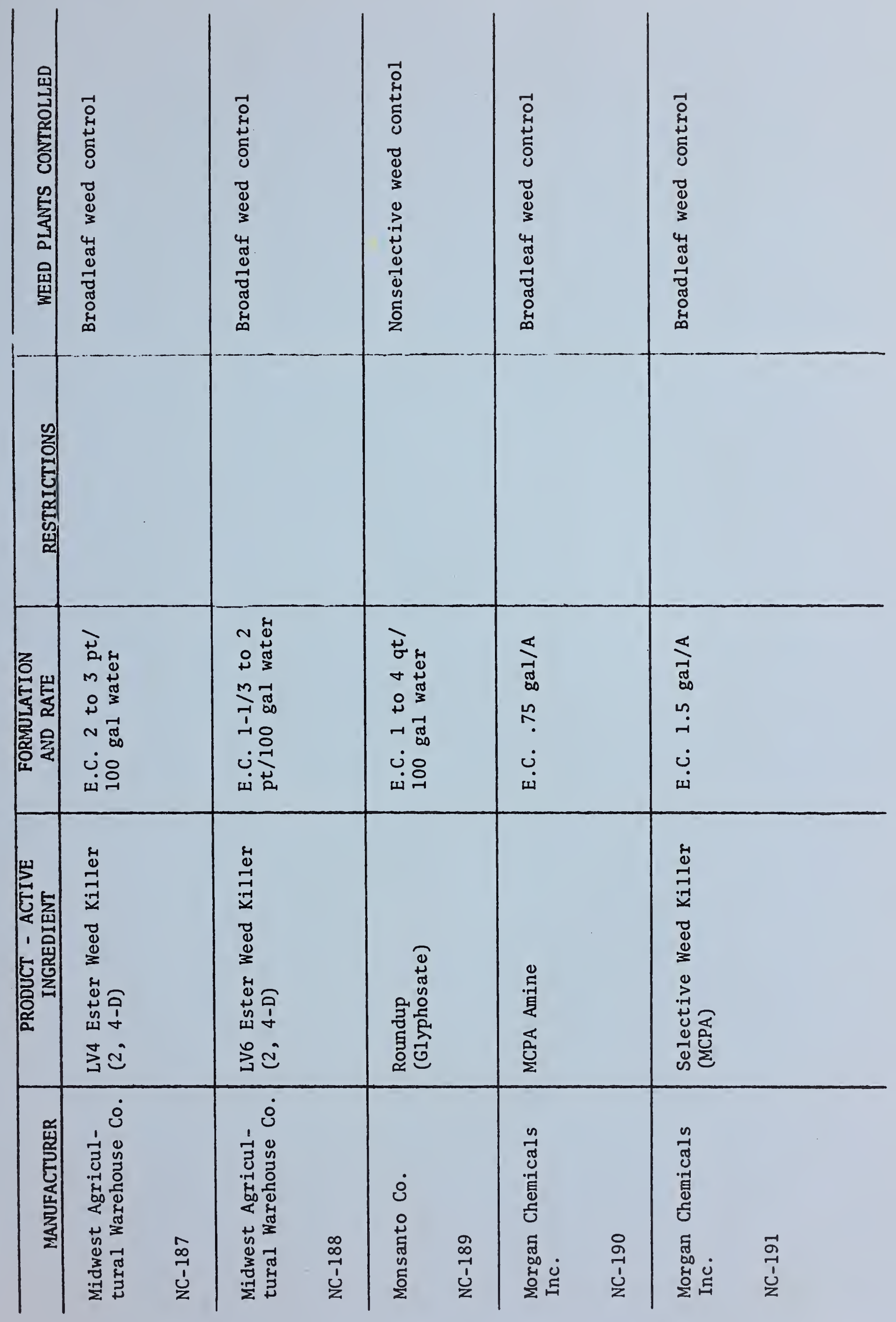




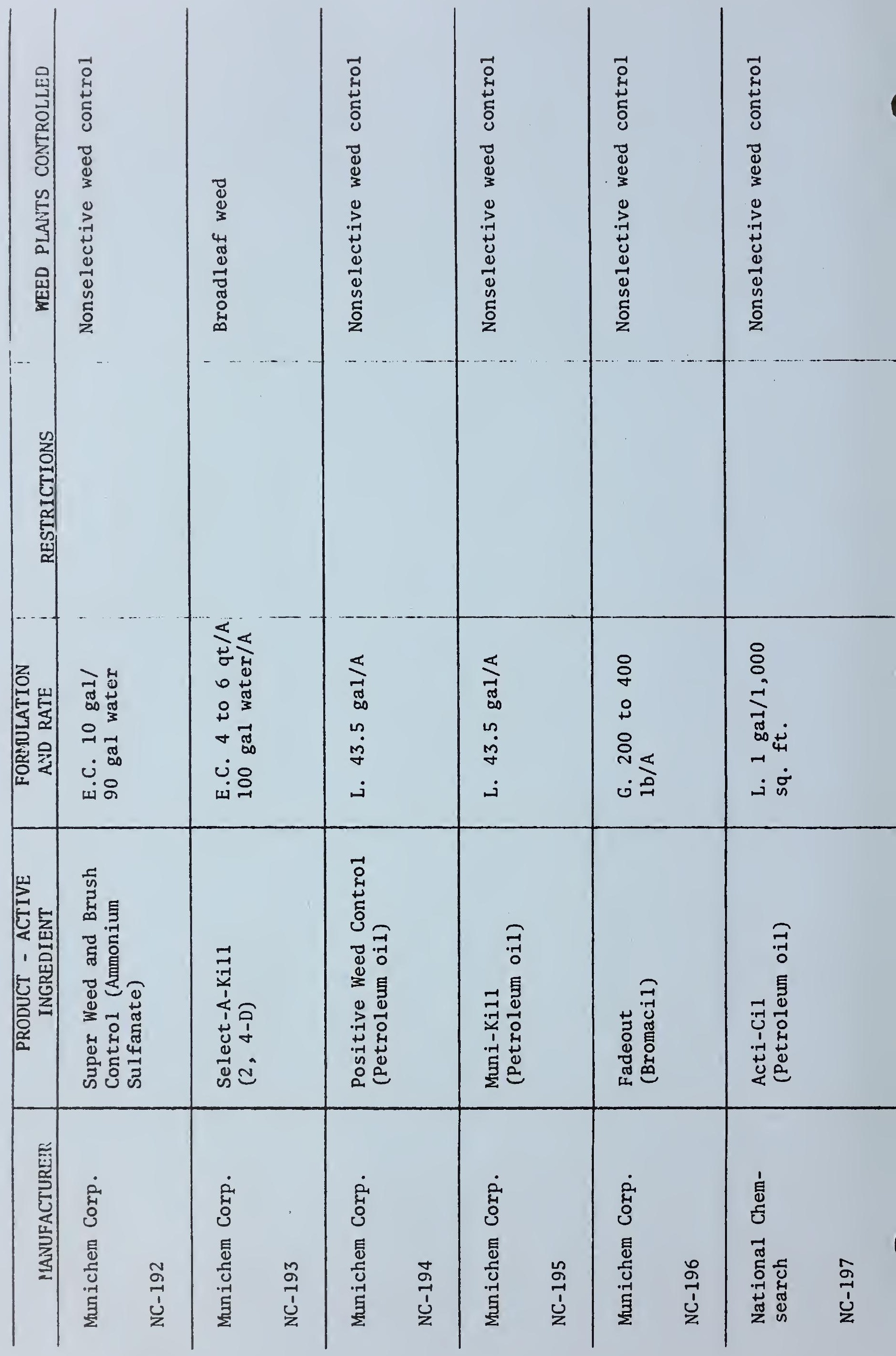




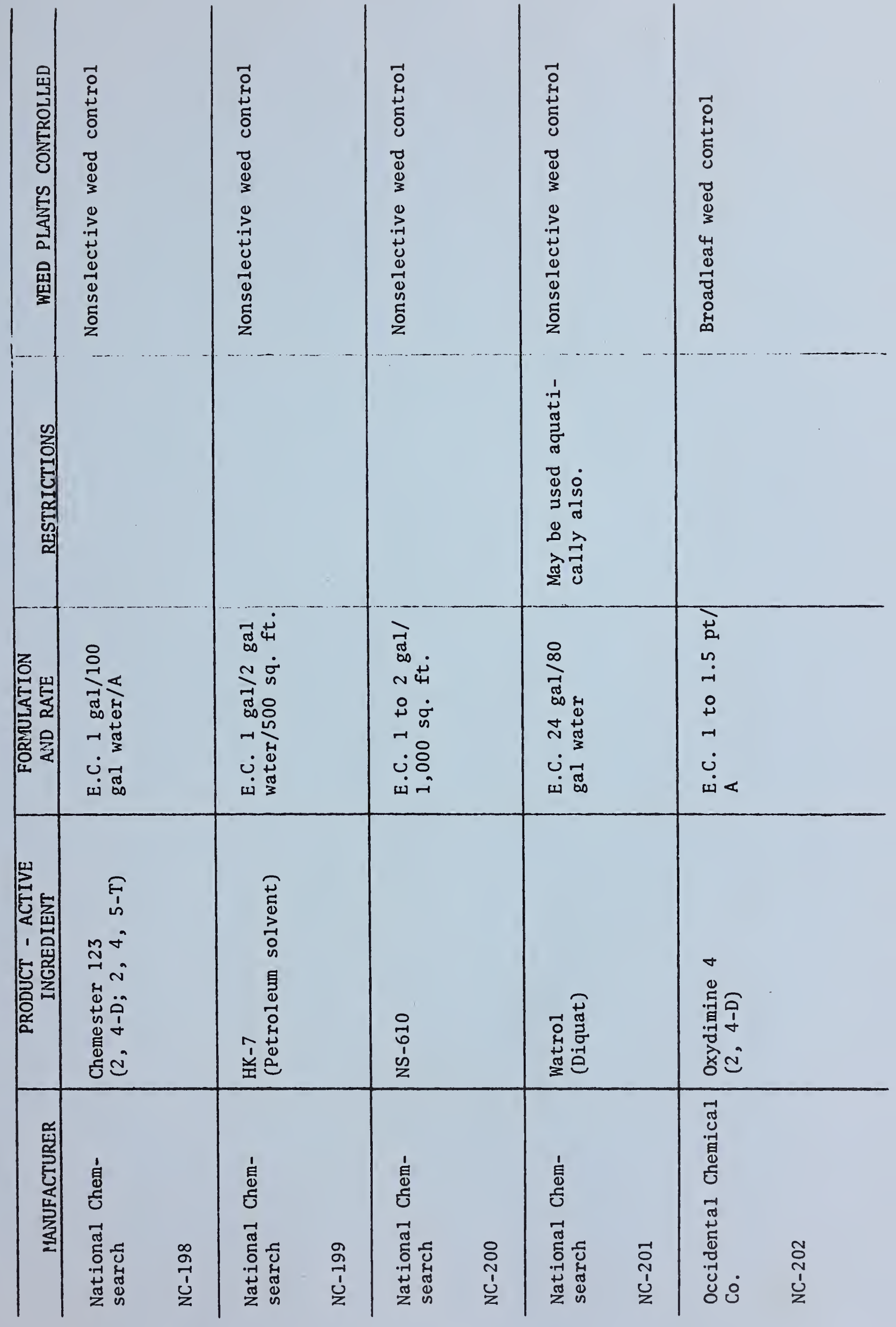




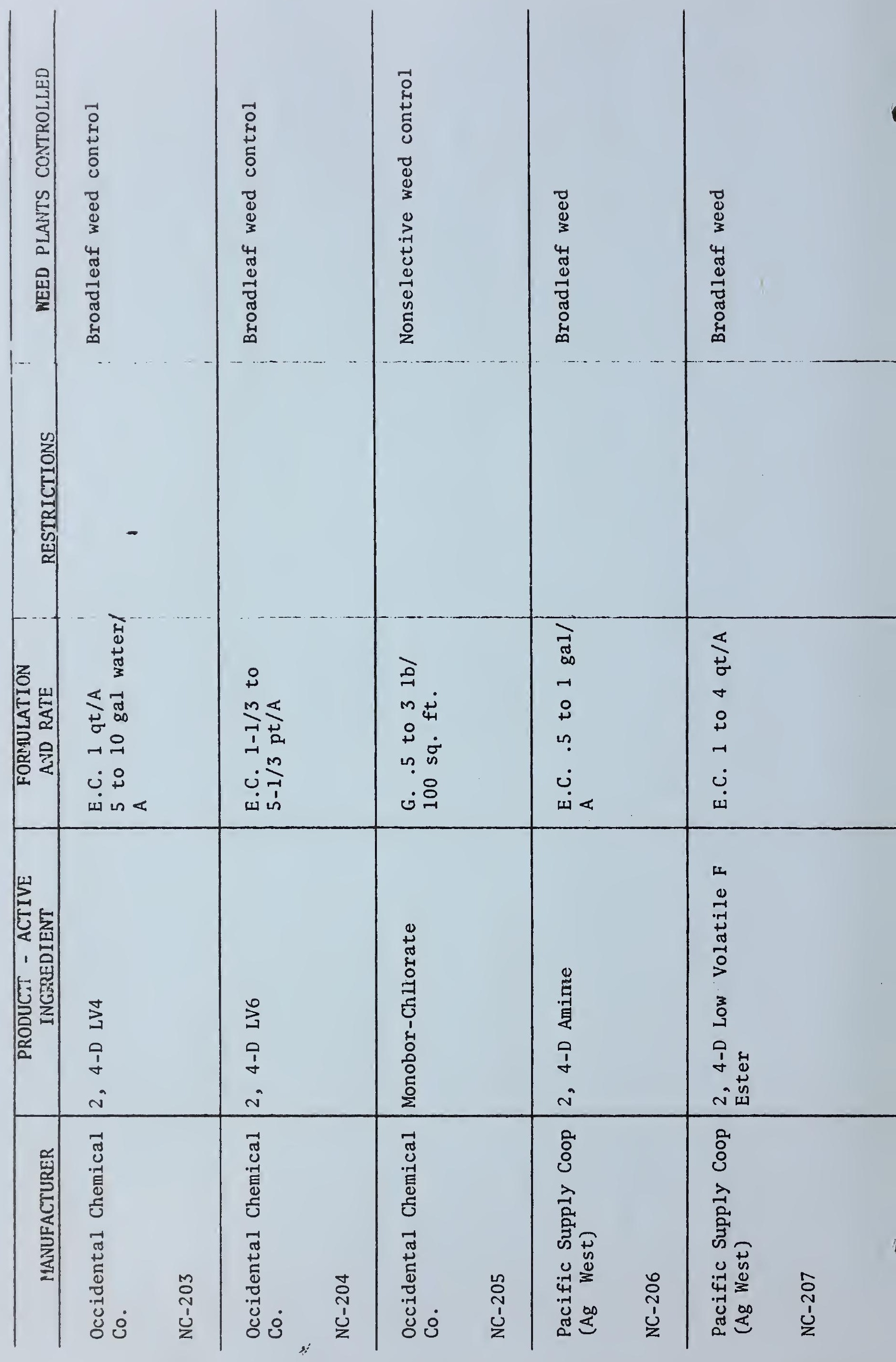




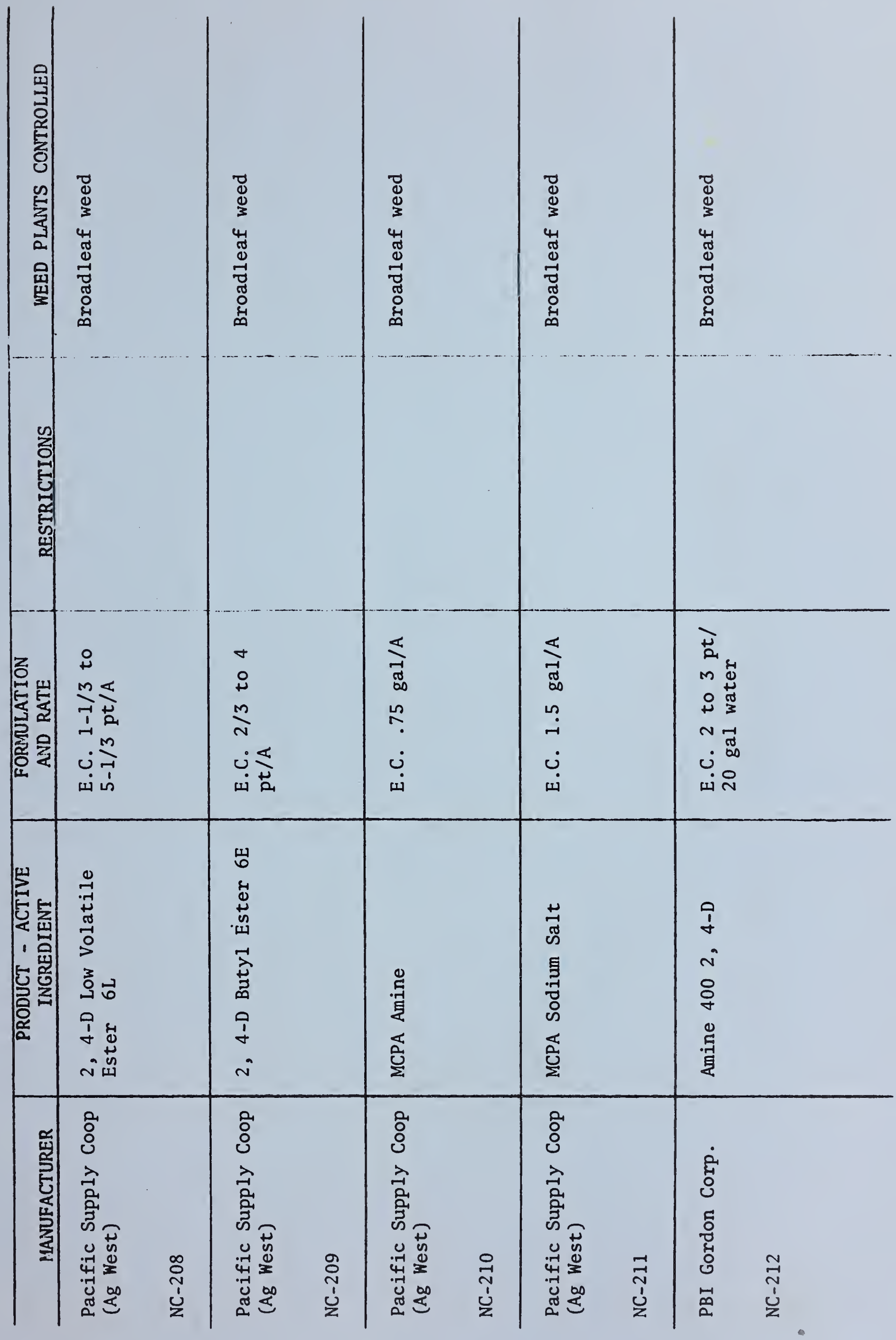




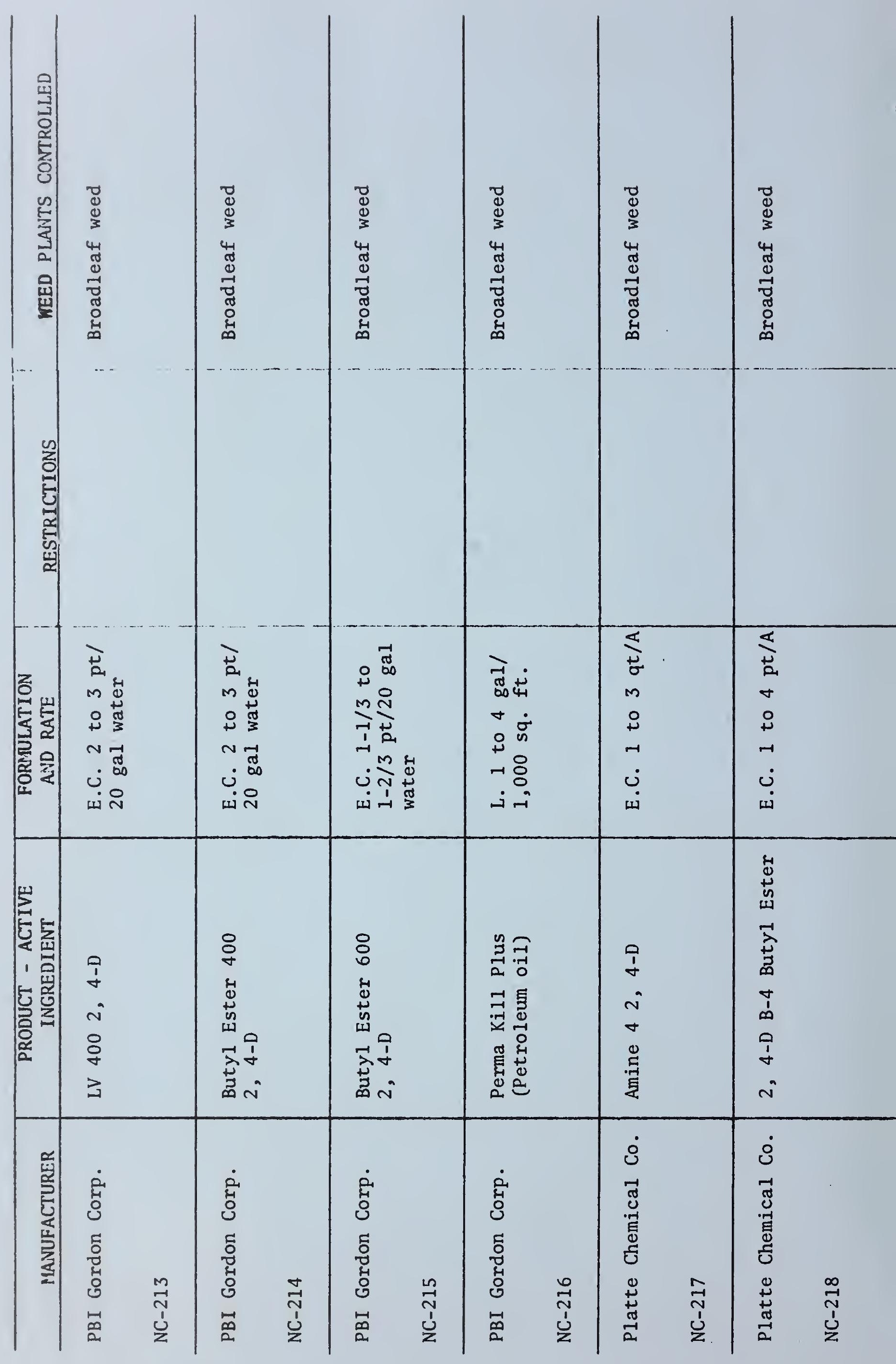




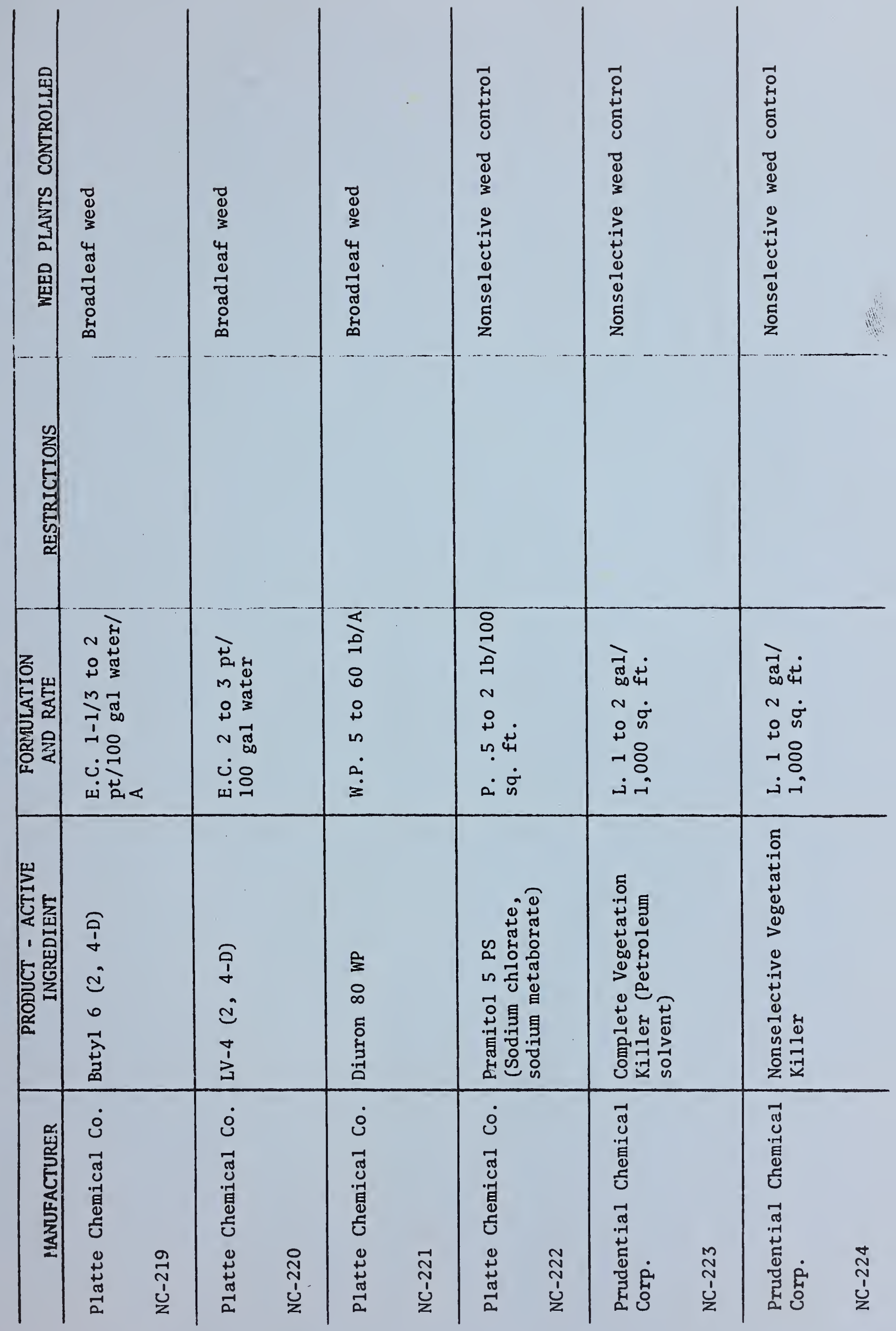




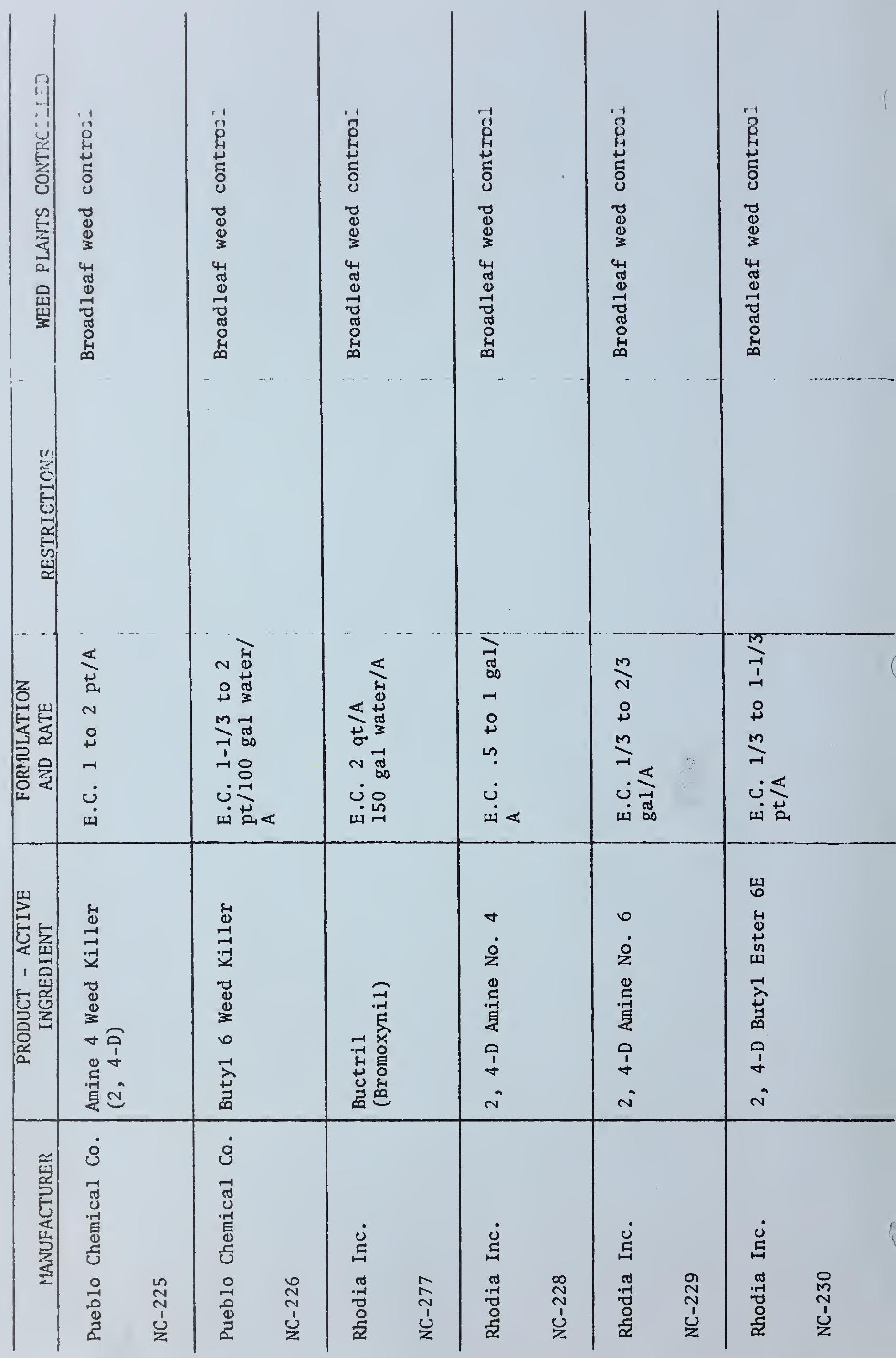




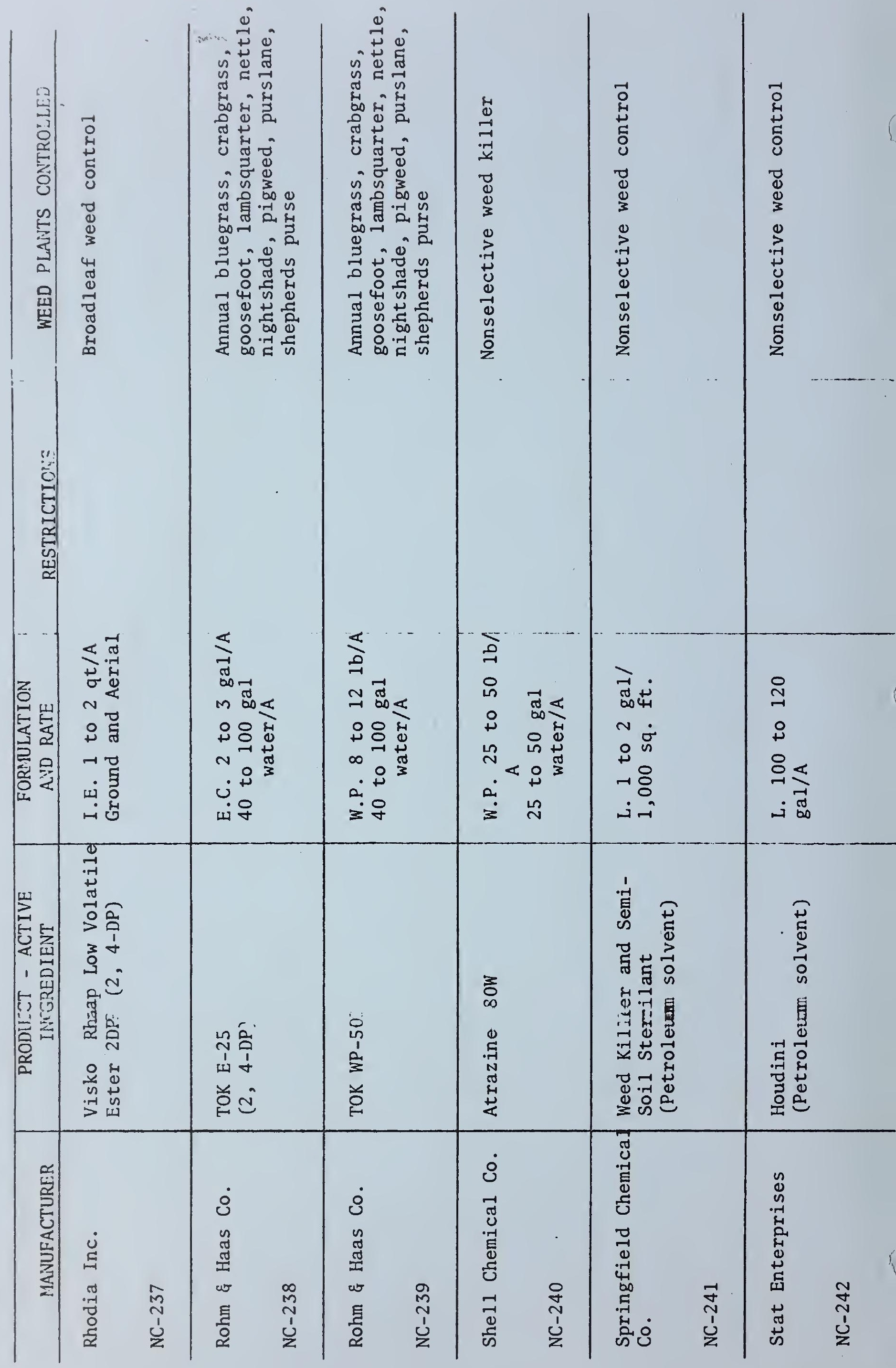




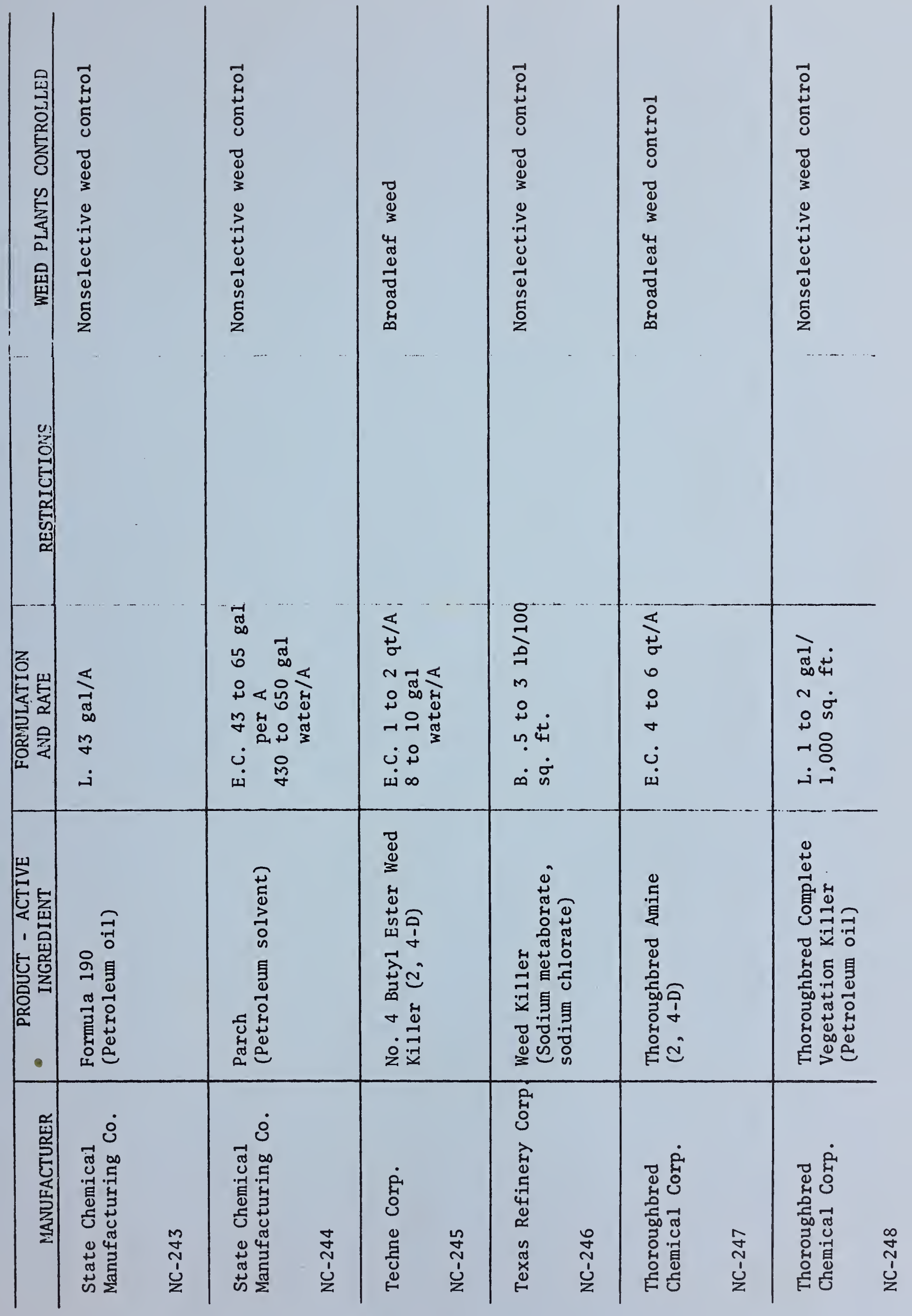




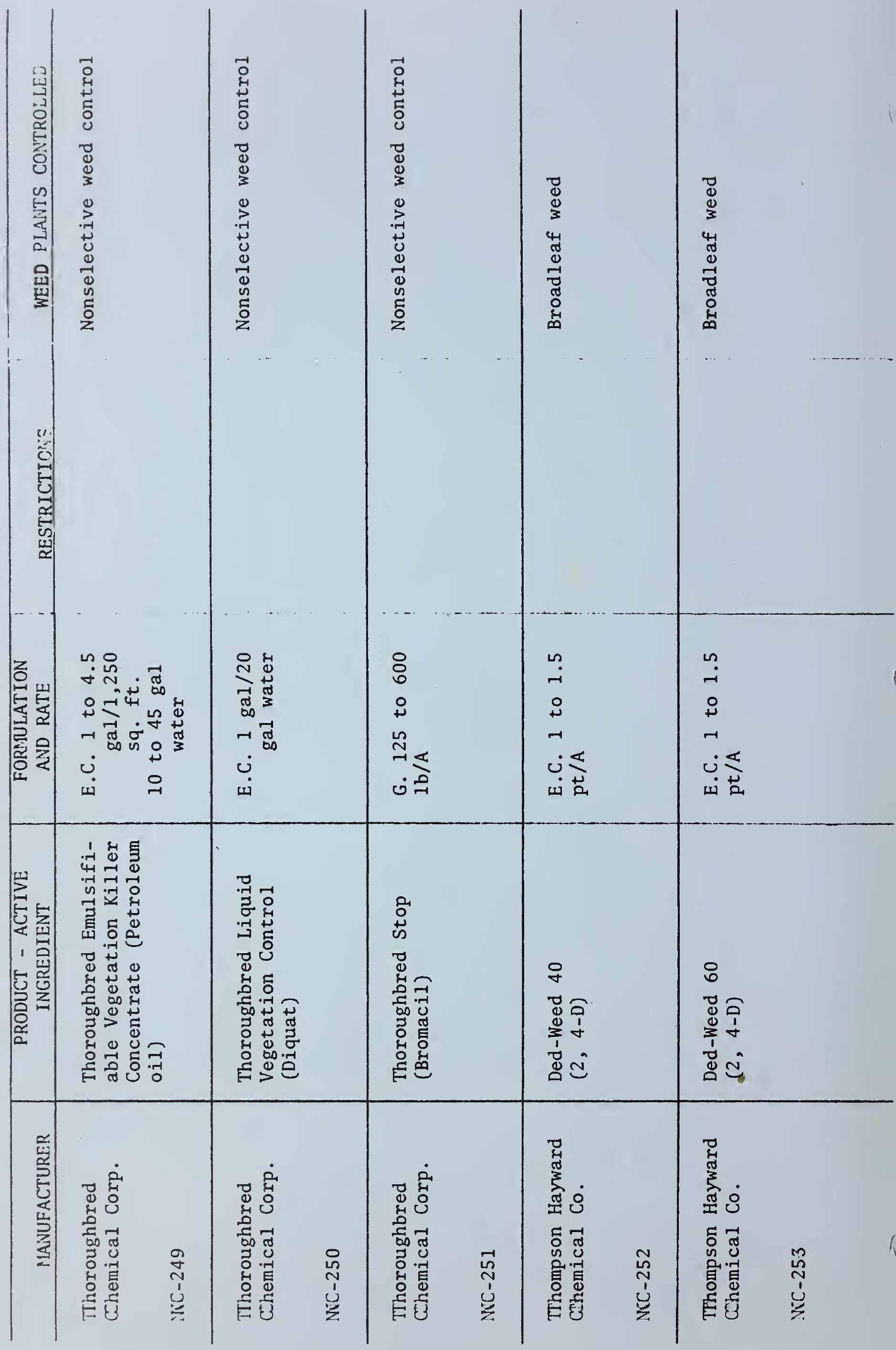




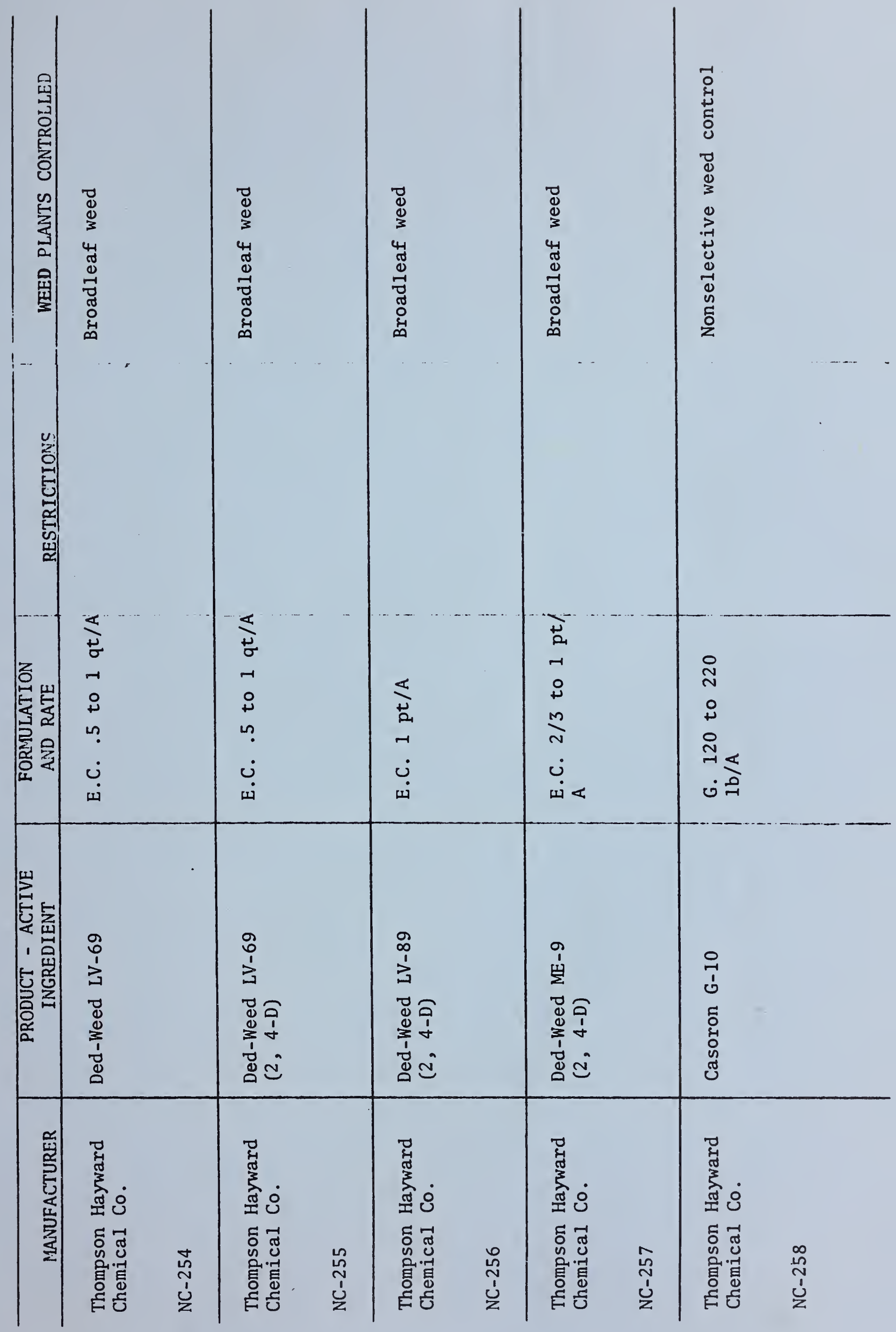




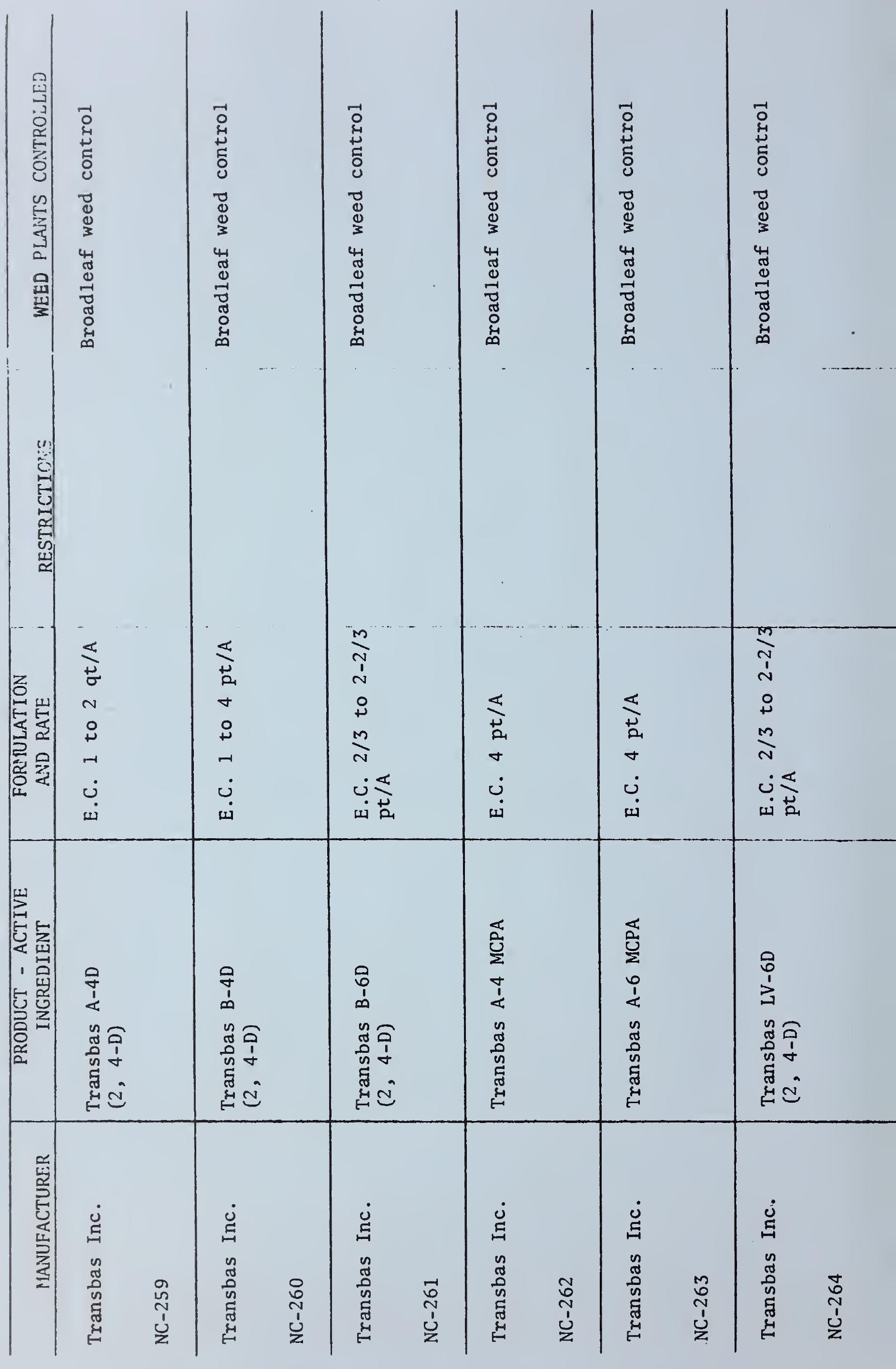




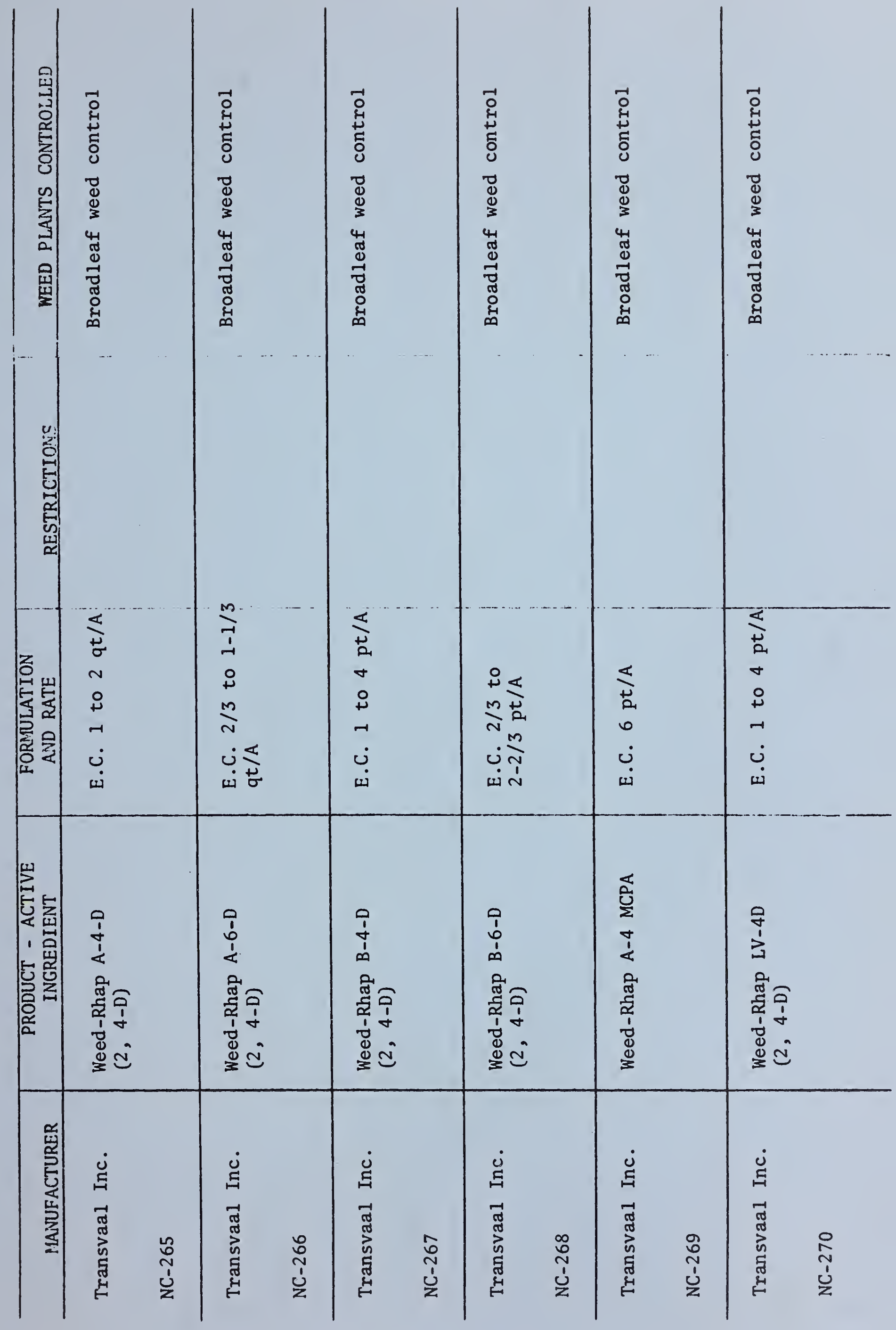




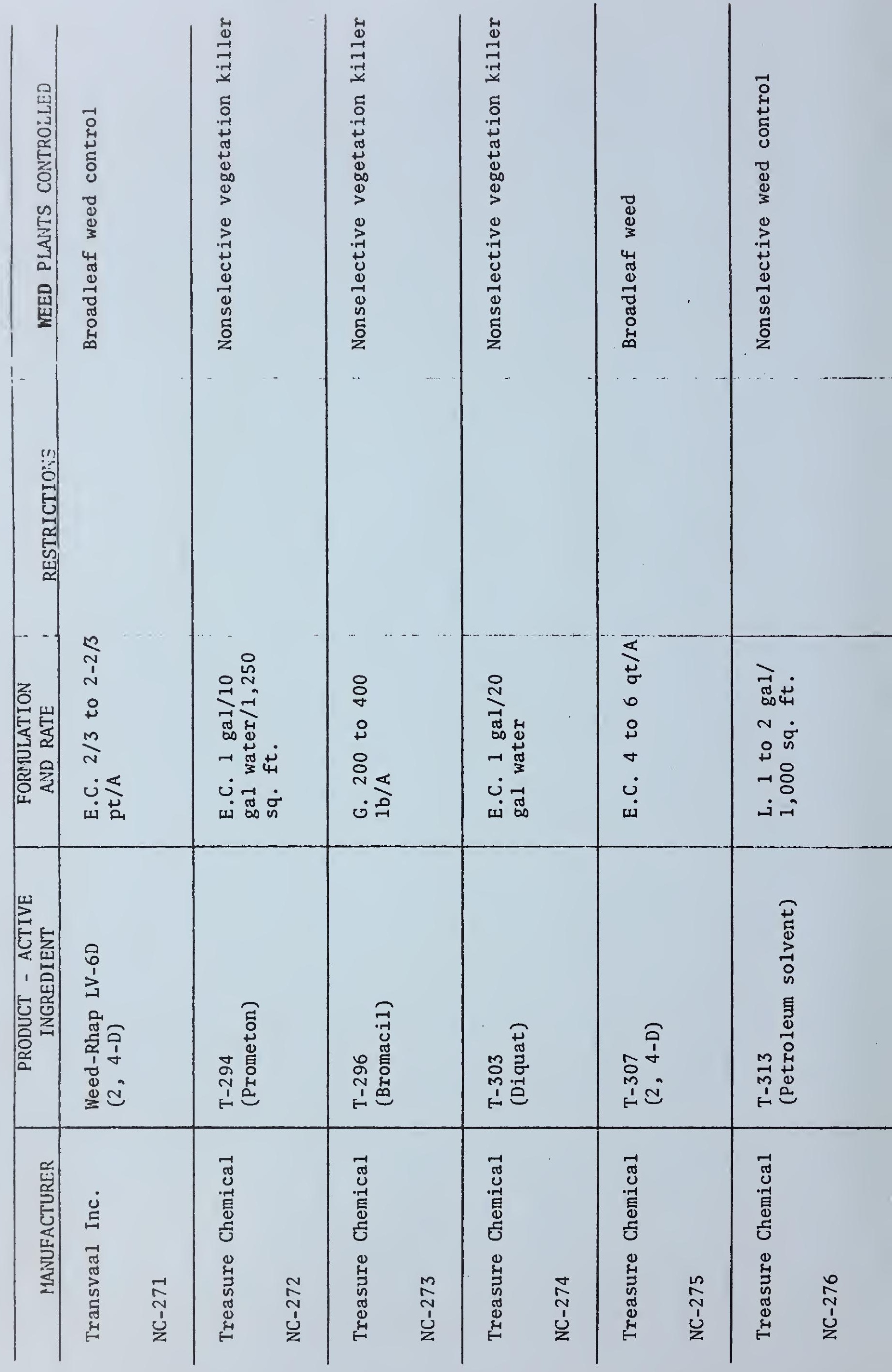




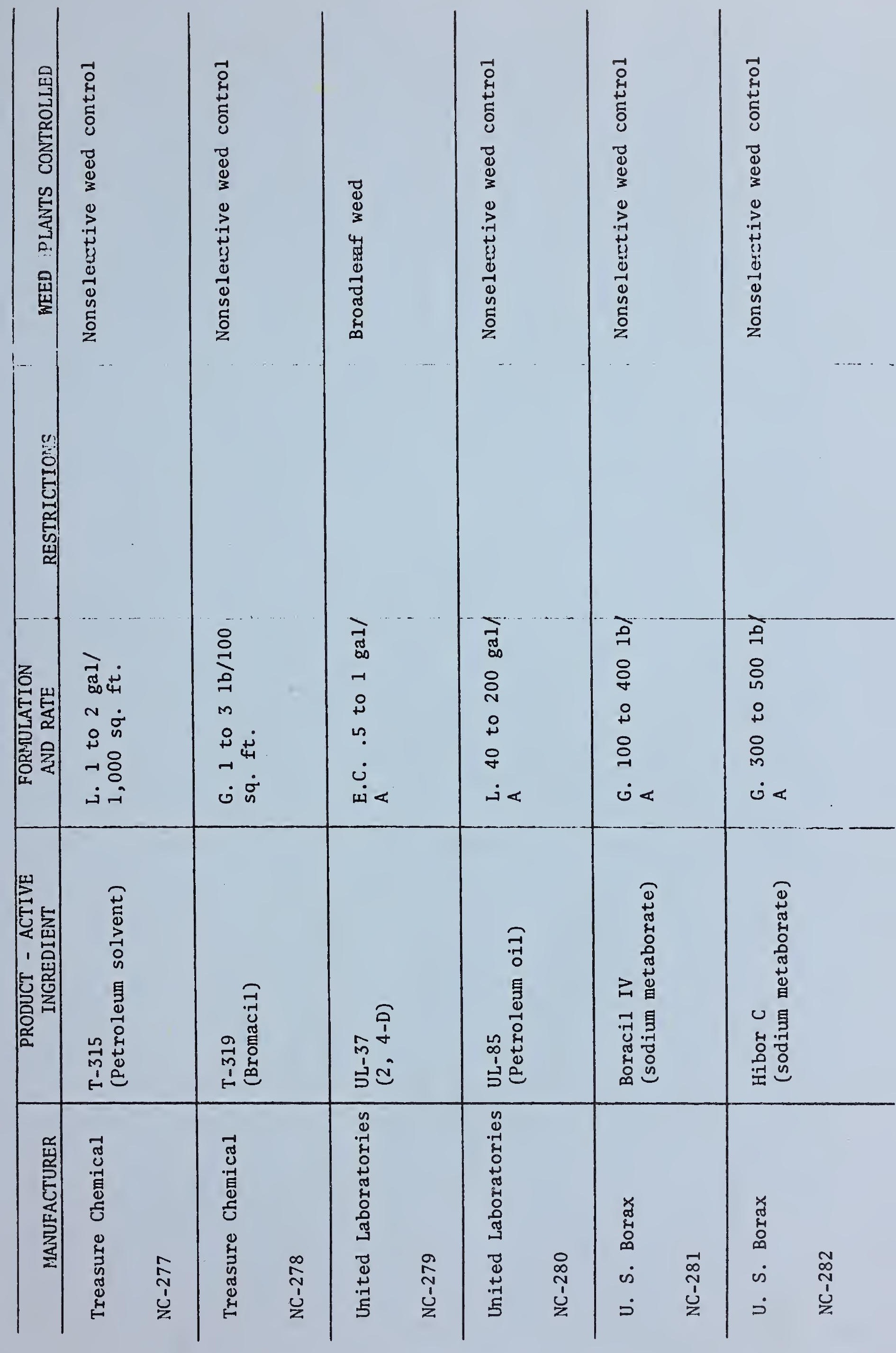




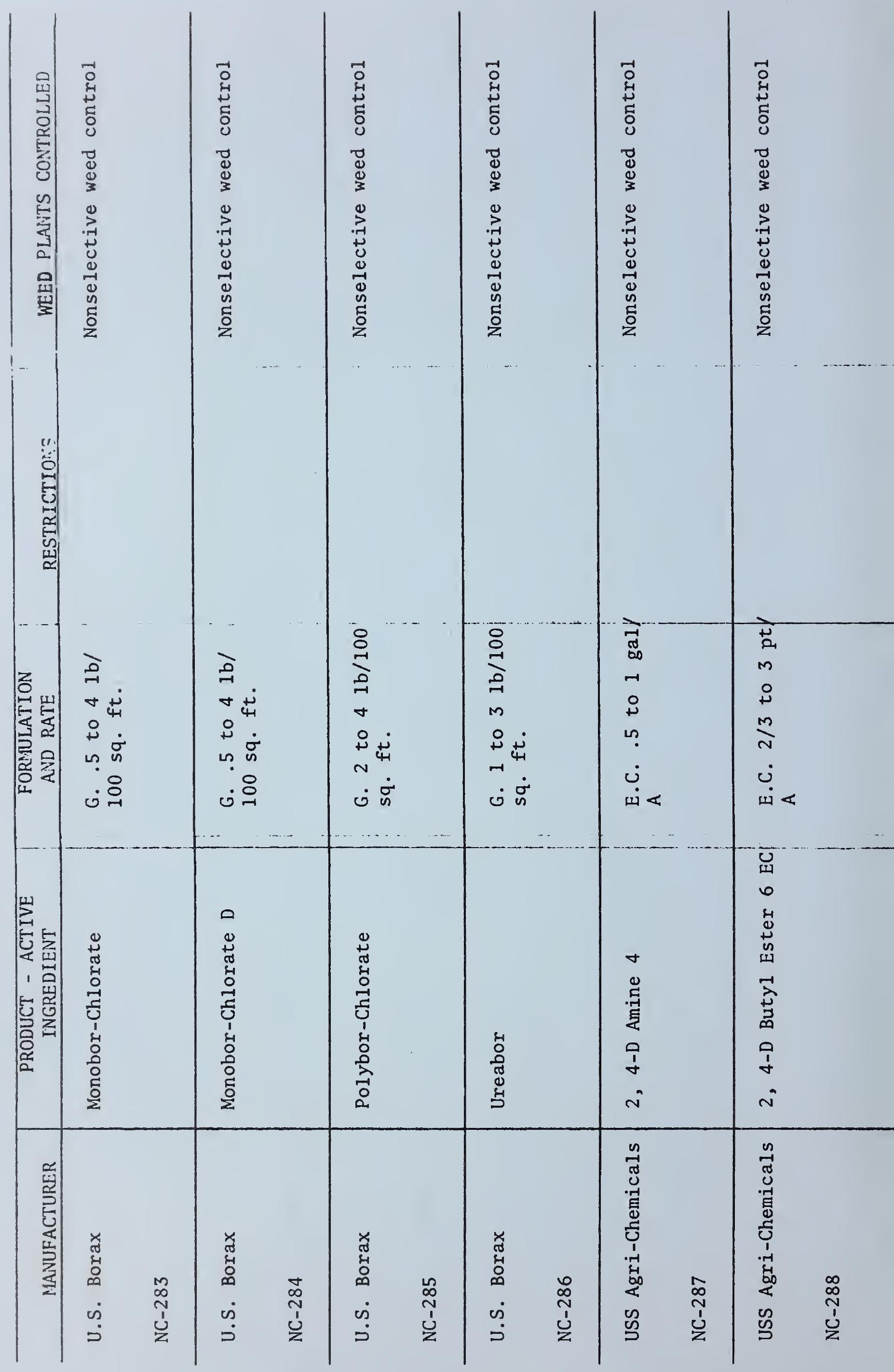




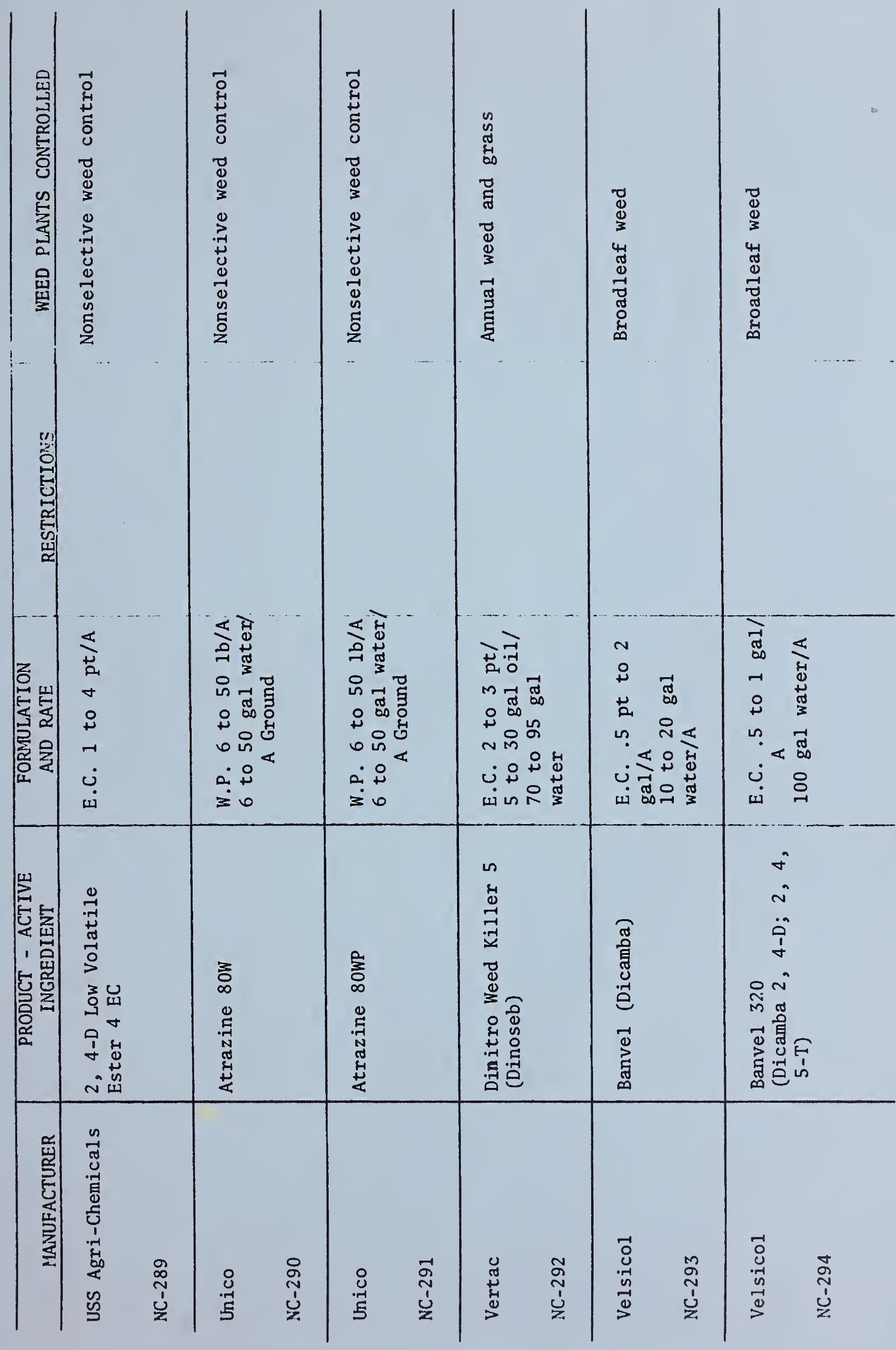




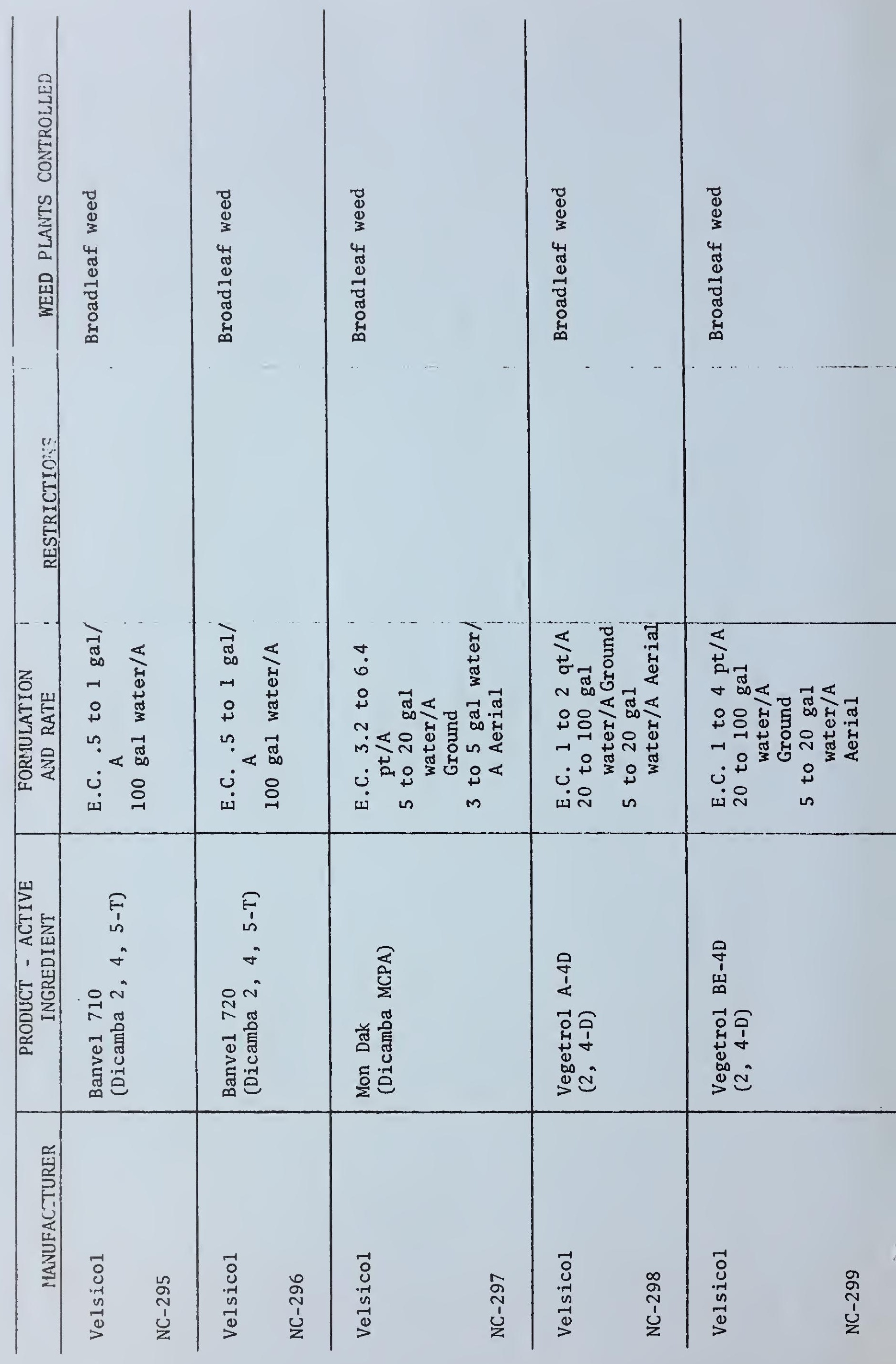




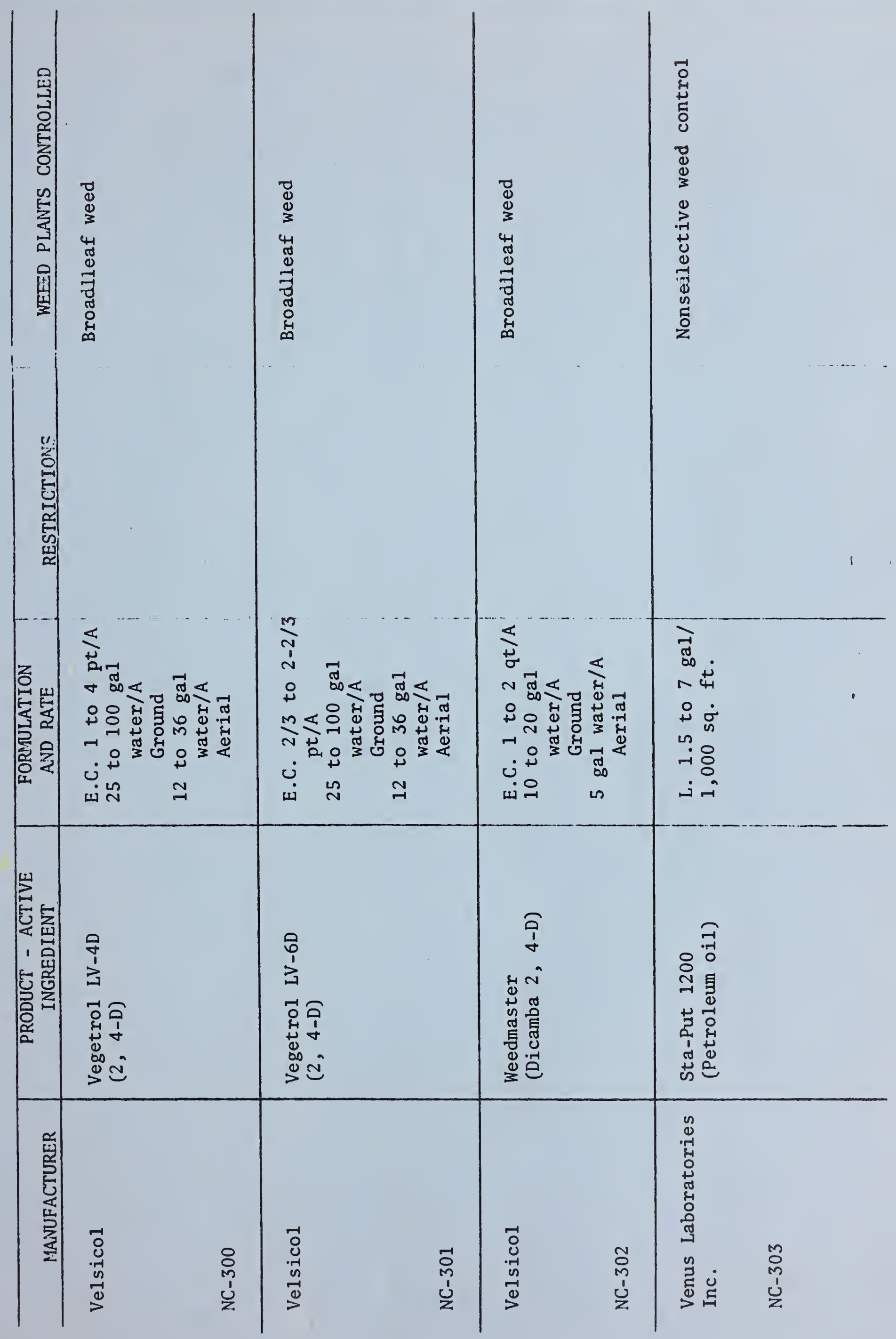




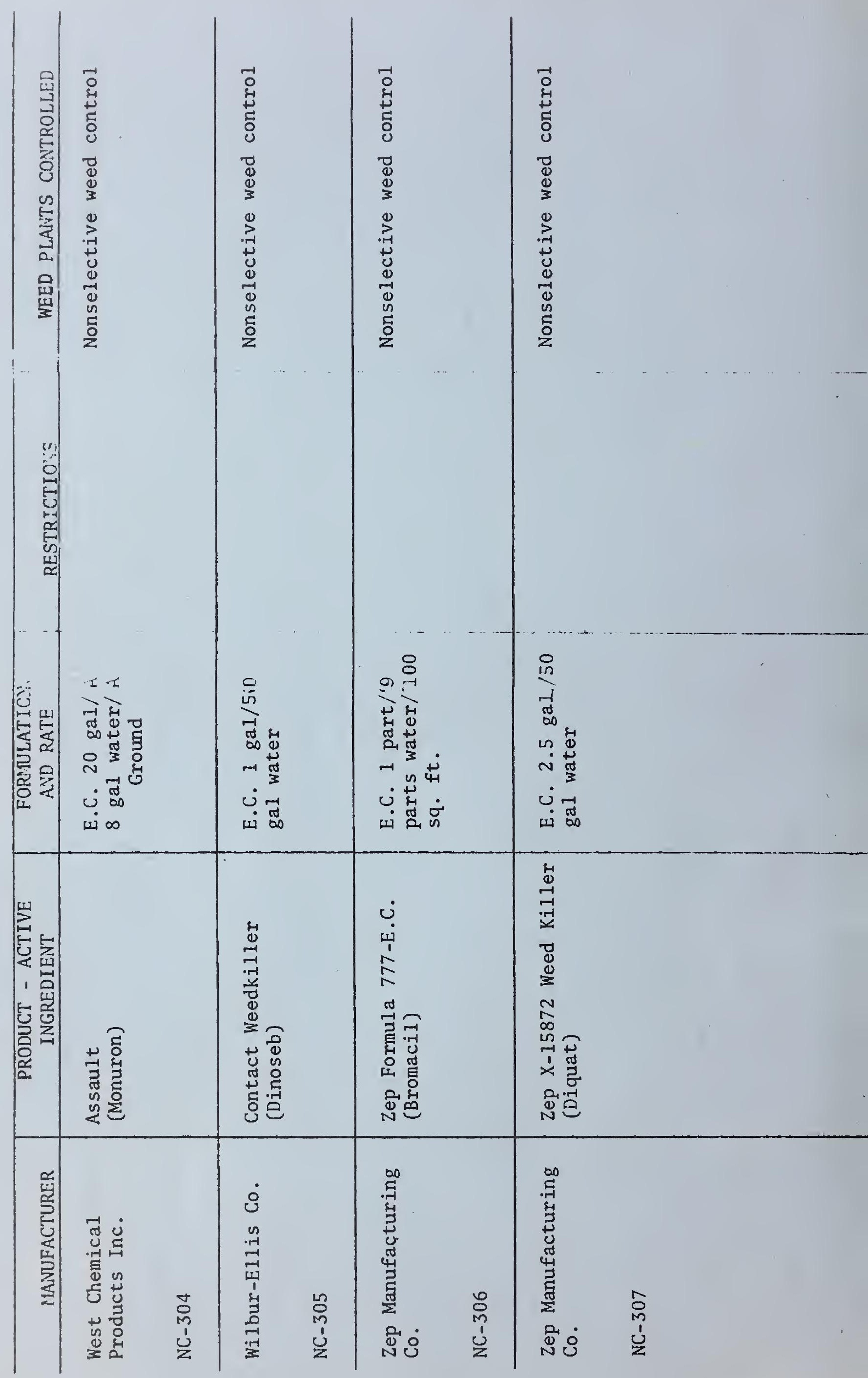




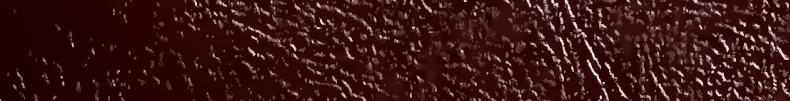

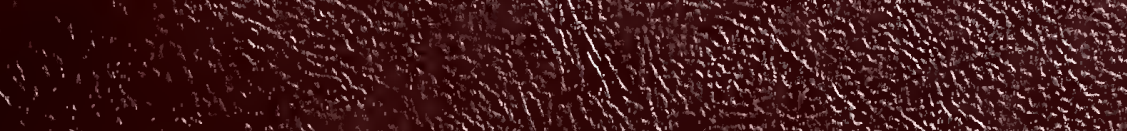

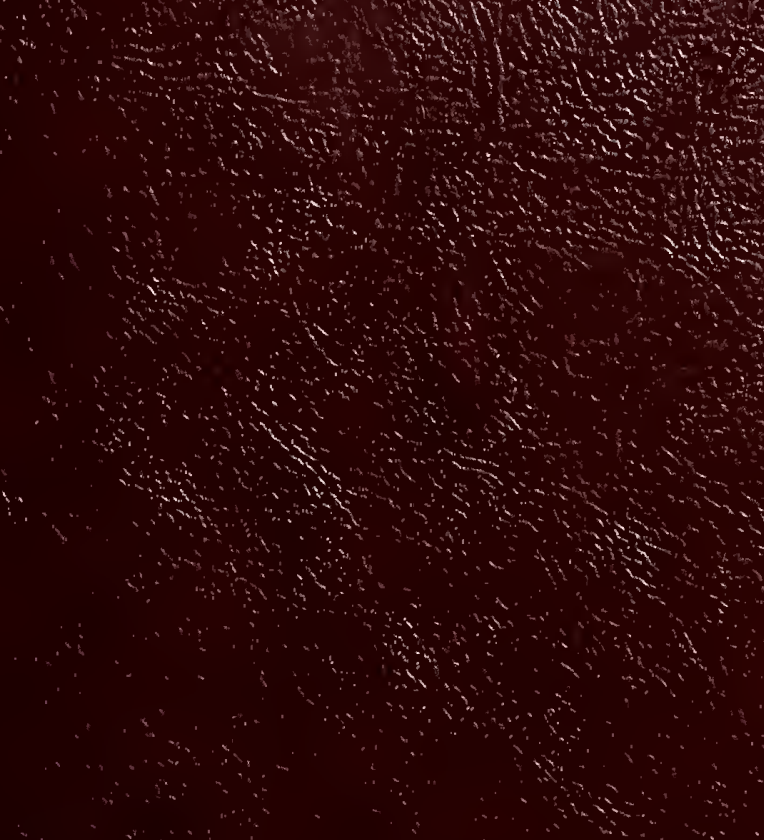

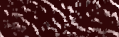
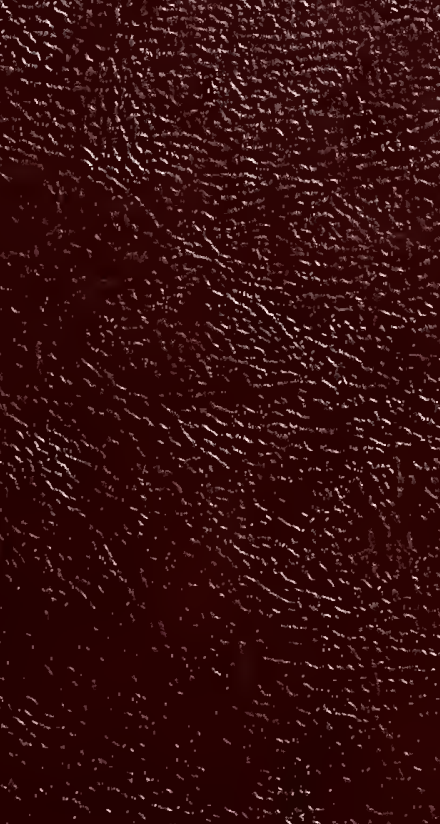

+

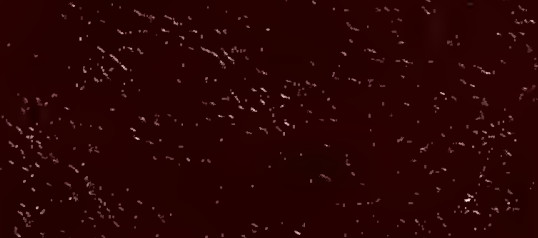

3 $+1$ (1) 1 a d ond ond -

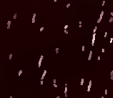


